
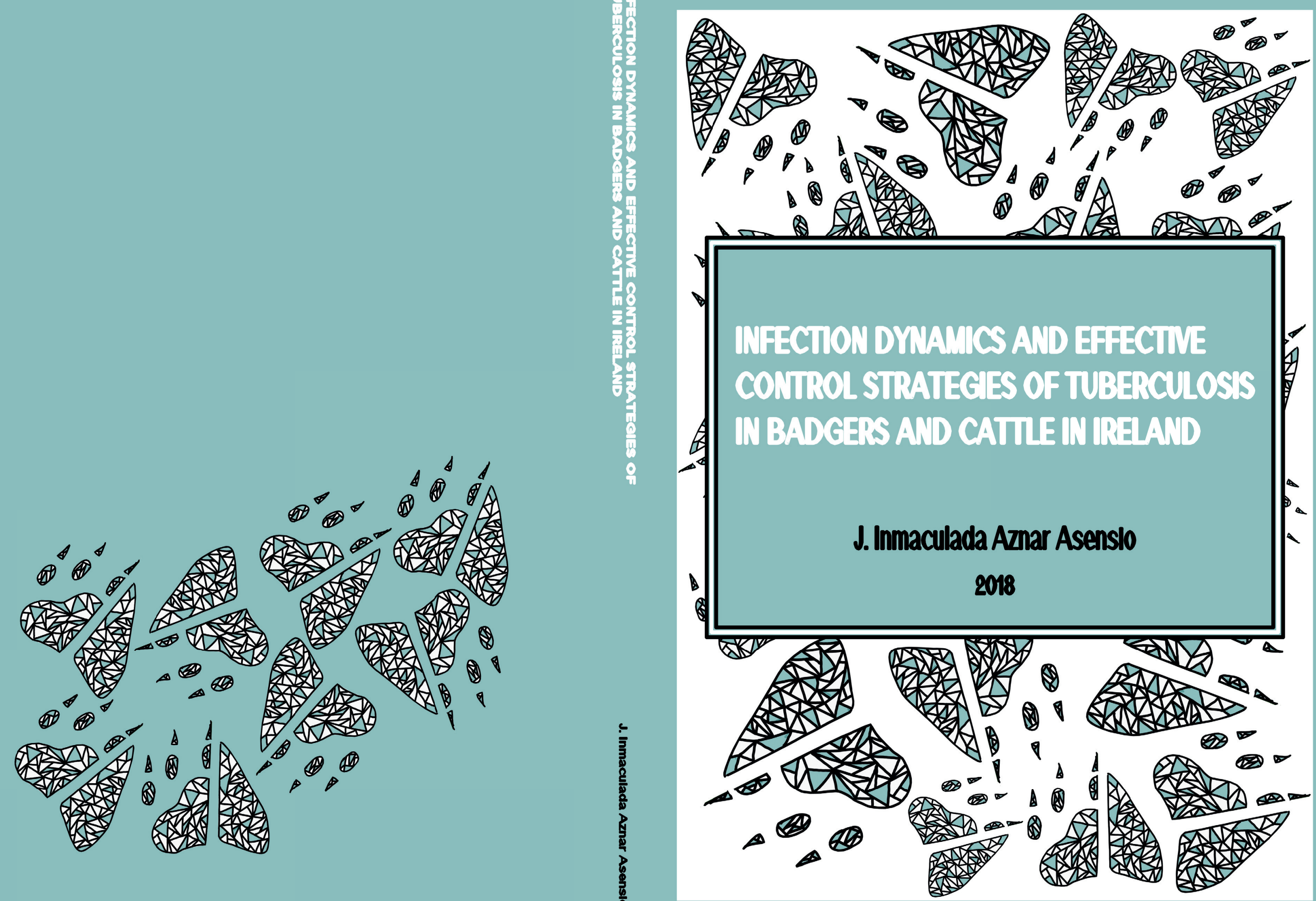

INFEGTION DYMAMTLS

ANI BFFGCTIY'

CONTROL STRATGGIBS

OF TUBGRCULOSIS IN

BADBGRS ANI GATILE

IV IRBLANI

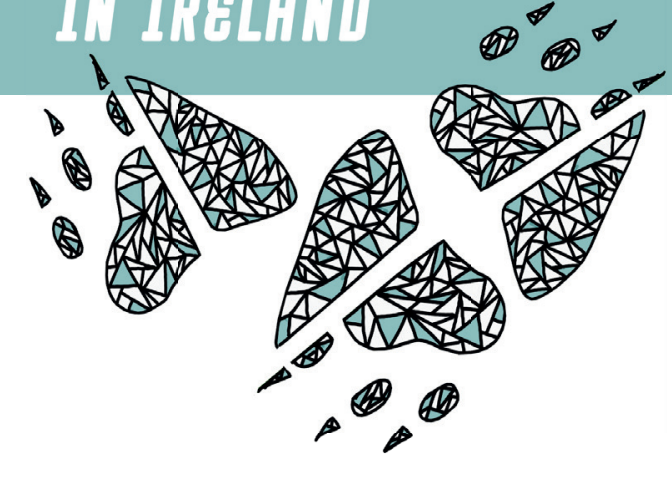

Friday

$9^{\text {th }}$ of February 2018

at 1.30 p.m. in the Aula

............

WAGENINGEN

UNIVERSITY

\& RESEARCH

J. Inmaculada Azuar Asenslo 


\section{Infection dynamics and effective control strategies of tuberculosis in badgers and cattle in Ireland}




\section{Thesis committee}

\section{Promotor}

Prof. Dr M.C.M. de Jong

Professor of Quantitative Veterinary Epidemiology

Wageningen University \& Research

\section{Co-promotor}

Dr K. Frankena

Associate professor, Quantitative Veterinary Epidemiology Group

Wageningen University \& Research

\section{Other members}

Prof. Dr M. Nielen, Utrecht University, The Netherlands

Dr A.P. Koets, Wageningen Bioveterinary Research, Lelystad, The Netherlands

Prof. Dr G.F. Wiegertjes, Wageningen University \& Research

Dr W.F. de Boer, Wageningen University \& Research

This research was conducted under the auspices of the Graduate School of Wageningen Institute of Animal Sciences (WIAS). 


\title{
Infection dynamics and effective control strategies of tuberculosis in badgers and cattle in Ireland
}

\author{
J Inmaculada Aznar Asensio
}

\author{
Thesis \\ submitted in fulfillment of the requirements for the degree of doctor \\ at Wageningen University \\ by the authority of the Rector Magnificus, \\ Prof. Dr A.P.J. Mol, \\ in the presence of the \\ Thesis Committee appointed by the Academic Board \\ to be defended in public \\ on Friday 9 February 2018 \\ at 1.30 p.m. in the Aula.
}


Aznar, I.

Infection dynamics and effective control strategies of tuberculosis in badgers and cattle in Ireland,

167 pages.

PhD thesis, Wageningen University, Wageningen, the Netherlands (2018)

With references, with summary in English

ISBN: 978-94-6343-726-4

DOI: https://doi.org/10.18174/430133 


\section{מש}

Chapter? I General introduction

Trial design to estimate the effect
of vaccination on tuberculosis incidence in badgers

9. Estimating the power of a Mycobacterium bovis 35 Chapter J vaccine trial in Irish badgers

Optimising and evaluating the characteristics

of a multiple antigen ELISh for detection of Mycobacterium bovis infection in a badger vaccine field trial

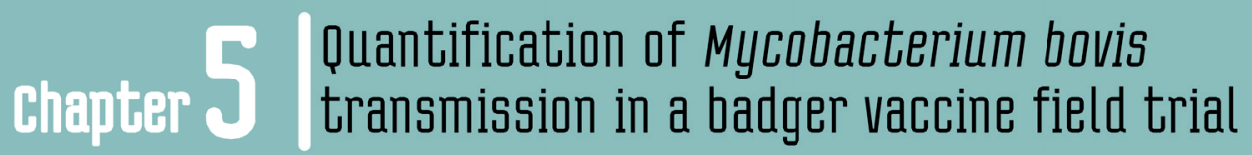

Chapter $6 \begin{aligned} & \text { Characterizing transmission of Mycobacterium } \\ & \text { bovis in a multi-host system }\end{aligned}$

Chapter 7 General discussion and conclusions

Chaptere 8 is summary

Training and supervision plan

Thesis Contributions 

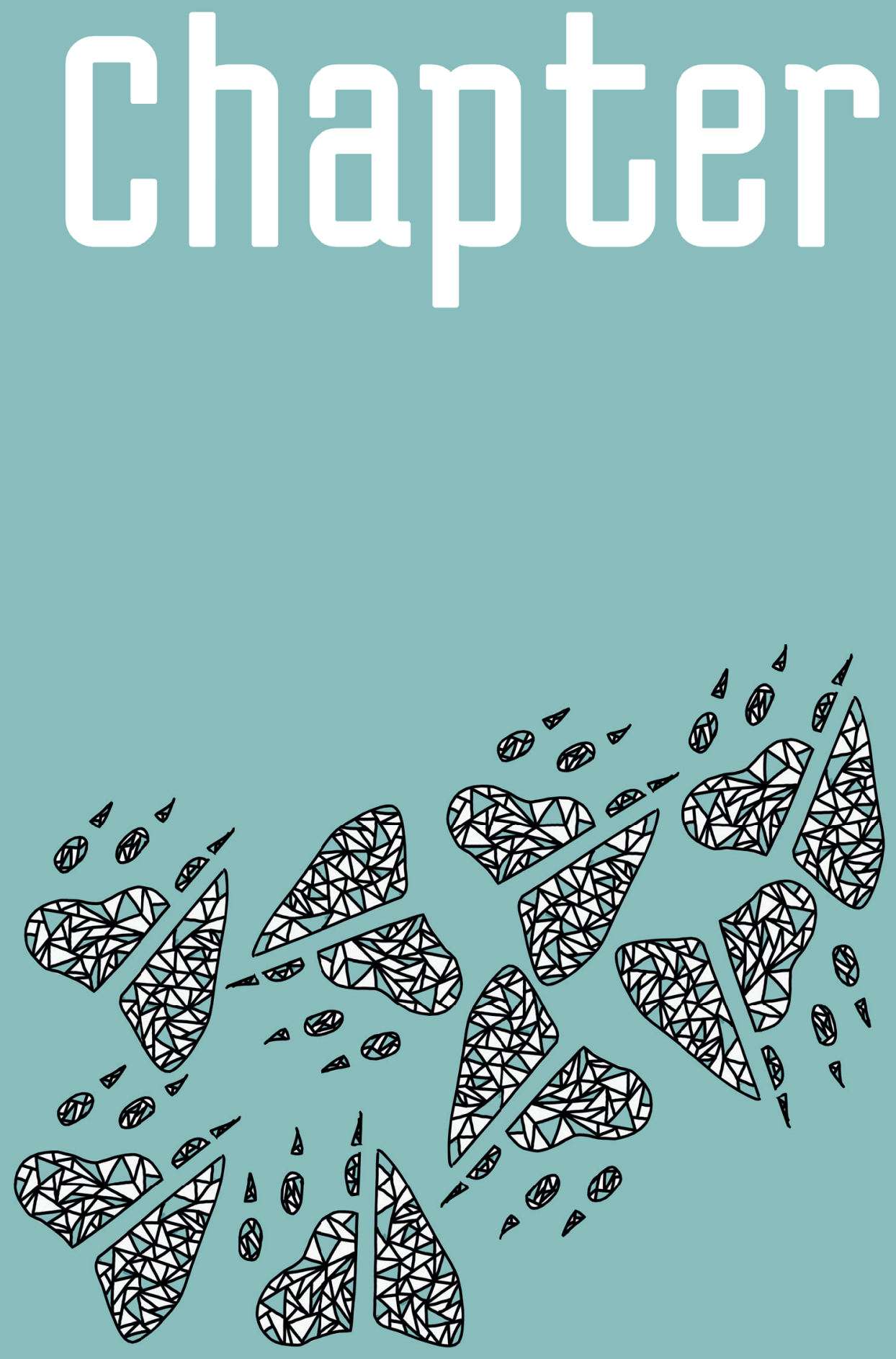


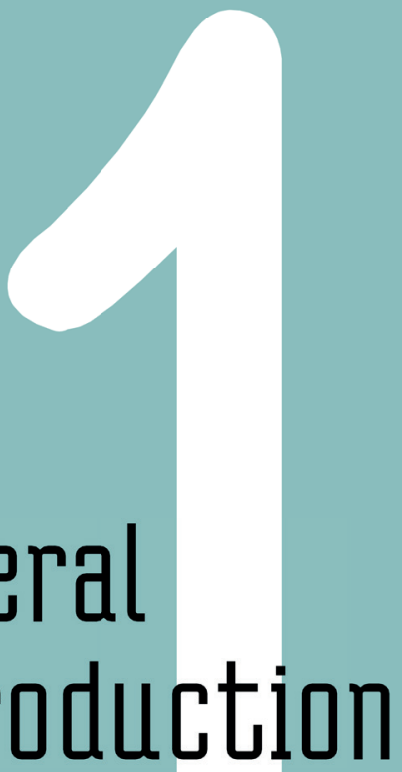




\section{Background information}

\section{$\underline{\text { Mycobacterium bovis (M. bovis) infection in cattle in Ireland }}$}

Mycobacterium bovis, the causative agent for tuberculosis in cattle, is a member of the Mycobacterium tuberculosis complex. This group is comprised of closely related bacterial species that cause tuberculosis in humans and other mammals (Wirth et al., 2008). Wildlife species acting as reservoir hosts for cattle include: brushtail possum, European badger, African buffalo, wild boar and white-tailed deer (Palmer, 2013). Bovine tuberculosis has a significant impact on farmer livelihood due to movement control and trading restrictions of affected herds, apart from the zoonotic aspect. In Ireland, an estimated $80 \%$ of the cattle herds were infected in 1954, when a control/eradication programme for $M$. bovis infection in cattle started. This programme initially consisted in the use of the Single Intradermal Comparative Tuberculin Test (SICTT) to test and remove infected cattle. Since this programme commenced, animal incidence decreased from $17 \%$ to $0.5 \%$ in 1965 (Watchorn, 1965; More and Good, 2006). The large success of this programme over a relatively short period of time led to a declaration of Officially Tuberculosis Free (OTF) status in accordance with Directive 64/432/EEC. Over the years and based on the findings of national and international scientific research, the Irish bTB control programme has been constantly under review, and additional control measures being progressively added. Apart from the intensive tuberculin testing of all cattle in the country on a yearly basis, many other measures such as random sample testing of herds, routine veterinary post-mortem surveillance at the slaughterhouse, contiguous testing, depopulation of problematic herds, etc. were adopted (More, 2005). Despite a strong focus on measures to reduce cattle to cattle transmission, prevalence of the disease has remained relatively stable since the initial decrease in animal incidence (More et al., 2006) as shown in Figure 1. 


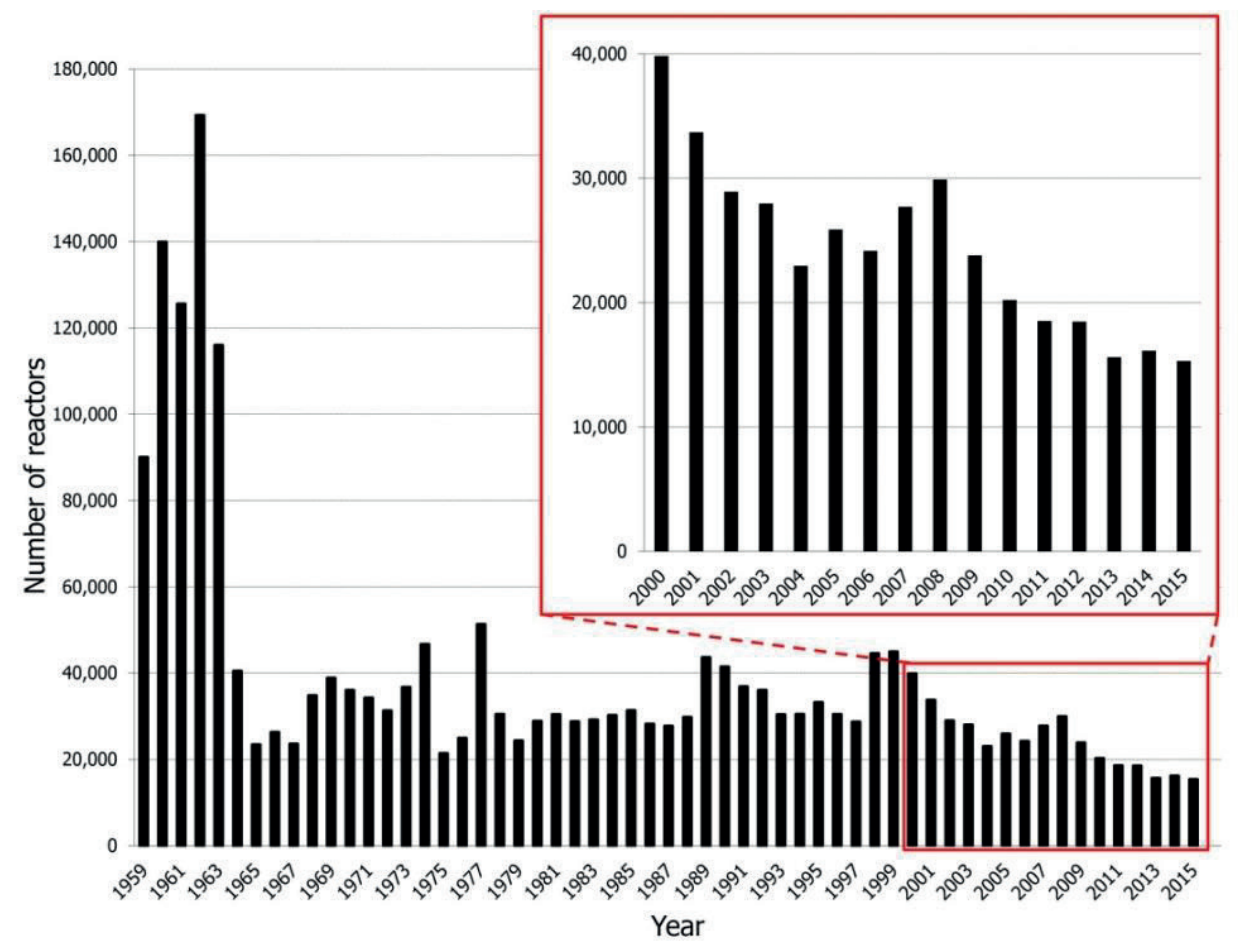

Figure 1. The annual number of reactors to the bovine tuberculin test in Ireland, from 1959 to 2015 (Updated figure from More, 2009).

\section{$\underline{\text { Badgers as a main reservoir for bTB }}$}

The inability to eradicate the disease by focusing on strategies that aimed at reducing cattle to cattle transmission suggested the presence of one or more reservoirs of the pathogen outside the cattle population. M. bovis was first isolated from a badger (Meles meles) in Ireland in 1974 (Noonan et al., 1975), following work carried out in the UK where badgers were also suspected as a reservoir for M. bovis (Krebs, 1997). Since then, a large amount of literature looking at different epidemiological aspects was dedicated to investigating the role of badgers in bTB maintenance (More and Good, 2006). In Costello et al. (1999), restriction fragment length polymorphism (RFLP) and spoligotyping analysis were used to strain type M. bovis isolates from domestic and wild species, showing a similar range and geographic distribution of strains from cattle, badgers, and deer. Several studies have highlighted the susceptibility of badgers to $M$. bovis infections, as well as a wide distribution of the 
infection in the badger population (Gormley and Costello, 2003; Corner et al, 2005). Two large field studies (the East Offaly and the four area projects) were conducted, seeking to clarify the role of badgers in the epidemiology of $M$. bovis infection in cattle. Following the proactive removal of badgers in some of the study area, these studies demonstrated a significant reduction in cattle incidence in comparison to control areas where no badger removals were carried out (Eves, 1999; Griffin et al., 2005; Olea-Popelka et al., 2009). These studies provided evidence of the role of badgers as a disease-maintenance-host (in areas of known cattle infection). It was then assumed that the main constraint to the eradication programme was the presence of a largely infected badger population.

In Ireland, badger culling commenced in 2000 as a strategy to reduce the incidence of the infection in badgers with the aim to achieve eradication in cattle. Since the introduction of culling, the incidence of bTB in cattle that had been stalled for the previous 30 years, commenced to slowly decline (small figure within Figure 1). Although a number of other strategies to tighten controls in cattle were also introduced during this time, based on the results obtained from field studies, it was believed that it was primarily badger culling that lead to this decline. The current national policy in response to a cattle herd bTB breakdown is to attempt to determine the most likely cause of the breakdown; if no specific cause can be identified, an investigation is carried out to establish whether or not badgers are responsible for transmission of infection. If badgers are the most plausible source of infection, then badgers from setts within a 500 meter radius from the infected farm are removed. A total of approximately 6,000 badgers are being culled per year as part of the DAFM wildlife bTB strategy. However, badgers are a protected species and as such, they have to be preserved to comply with the Bern Convention (Convention on the Conservation of European Wildlife and Natural Habitats, 1979). As badger culling cannot be sustained over time, research has gradually moved towards investigating a feasible long-term solution. Bacille Calmette-Guérin (BCG) vaccination in badgers was an eradication strategy on which all stakeholders (policy makers, farmers, ecologists, economists, and society) could agree.

\section{$\underline{\text { BCG badger vaccination }}$}

A 10-year project to study the possibility of using BCG vaccine in badgers to assist in the control and eradication of bTB in cattle commenced in Ireland in 2001 funded by the Department of Agriculture, Food and the Marine (DAFM). A number of experimental challenge trials were initially conducted with captive badgers (Corner et al., 2007; Lesellier et al., 2009). In all of these studies, vaccination via either subcutaneous, mucosal, oral or intramuscular routes showed a reduction of disease progression (Corner et al., 2008; Lesellier et al., 2009, 2011; Murphy et al., 2014). The findings from these trials were promising, and helped to refocus the scientific 
debate towards the need to assess estimates of BCG vaccine efficacy and its effectiveness in terms of reducing $M$. bovis transmission in natural populations of badgers.

\section{Thesis Objectives}

\section{$\underline{\text { Vaccine Efficacy estimation }}$}

The first objective of this thesis was to design a field trial enabling quantification of the efficacy of BCG vaccination on $M$. bovis transmission in badgers under field conditions. BCG had previously been tested in badgers in a controlled environment; however, it is not possible to directly extrapolate these results to vaccine efficacy under field conditions. To that purpose, the Kilkenny badger vaccine field trial was designed (Aznar et al., 2011). Although laboratory trials had shown a reduction of disease progression in captive badgers, all badgers challenged with $M$. bovis became infected. A field trial was therefore a way of establishing whether or not protection against infection could be attained under natural conditions, where the infective dose that badgers are exposed to is likely much lower than that used in experimental trials (Corner et al., 2010; Lesellier et al., 2011; Aznar et al., 2011). Protection against infection can be the result of both a reduction in the susceptibility of the receiver and/or a reduction in the infectiousness of the donor. Reduction in susceptibility corresponds first of all to a direct effect of vaccination or vaccine efficacy for susceptibility $\left(\mathrm{VE}_{\mathrm{S}}\right)$. Note, however that a reduction in susceptibility also has an indirect effect, as it reduces the transmission of bTb. Likewise, the trial in wild badgers could help to determine if the observed reduction in disease progression under laboratory conditions was linked to a reduction in infectiousness and therefore in the probability of secondary transmission. This type of protection is referred to as vaccine efficacy for infectiousness $\left(\mathrm{VE}_{\mathrm{I}}\right)$. In this thesis we aimed to estimate both $\mathrm{VE}_{\mathrm{S}}$ and $\mathrm{VE}_{\mathrm{I}}$.

\section{Estimation of the basic reproduction ratio $\left(\mathrm{R}_{0}\right)$ and $\mathrm{R}(\mathrm{p})$}

The second and most important objective in this thesis was to assess how much reduction in $M$. bovis transmission in badgers, and subsequently in the cattle-badger system, could be achieved by introducing BCG badger vaccination. To measure the impact of vaccination in transmission, first it was essential to have an estimate of the current level of transmission as expressed by $\mathrm{R}_{0}$. $\mathrm{R}_{0}$ is a mathematical parameter that refers to the number of cases one typically infectious individual generates, on average, over the course of its infectious period in a fully susceptible population. $\mathrm{R}_{0}$ is a very important parameter, summarizing the behaviour of an infection in a population in just one single value (Kermack et al., 1927; Diekmann et al, 1990, 2010; Fulford et al., 2002). This parameter is very simple to interpret when used as 
a threshold. If for the cattle and badger system in Ireland $\mathrm{R}_{0}$ under the current control strategies is $<1$, bTB will die out in the long run, if $\mathrm{R}_{0}>1 \mathrm{bTB}$ eradication with current control measures would not be achieved.

Once the $\mathrm{R}_{0}$ for the system is estimated under current control measures, the efficacy of added strategies such as badger vaccination could be assessed based on how much they are capable of reducing $\mathrm{R}_{0}$, ideally to below 1 . As $\mathrm{R}_{0}$ is a combination of the susceptibility of the receiver and the infectivity of the donor, knowing the effects of vaccination in both susceptibility and infectivity was essential to estimate the efficiency of vaccination. The $\mathrm{R}_{0}$ for the $M$. bovis cattle-badger system had never been estimated in Ireland. In this thesis, an estimate of the $\mathrm{R}_{0}$ for the system is presented as well as how this parameter changes depending on vaccination coverage $(\mathrm{R}(\mathrm{p})$ ). Also, by estimating the Next Generation Matrix (NGM) for the system, we aimed to assess the contributions of each of the species transmission to the system $\mathrm{R}_{0}$ and the reduction of this parameter and the partial reproduction ratios achieved by $\mathrm{BCG}$ vaccination.

\section{Chapters and challenges}

Chapter 2: Trial design to estimate the effect of vaccination on tuberculosis incidence in badgers.

The design of the Kilkenny badger vaccine field trial, as well as the methodology to analyse data generated from it, are presented in the second chapter of this thesis. The challenges in designing a trial capable of estimating both the direct and the indirect effect of vaccination have been highlighted in the literature (Halloran et al., 1997, 1999; Longini et al., 1998). In essence, while a double blinded randomized vaccine placebo controlled trial is still the design of choice to estimate $\mathrm{VE}_{\mathrm{S}}$, it does not allow estimations of $\mathrm{VE}_{\mathrm{I}}$ unless information on actual contacts between infected and susceptible individuals is available. Longini et al. (1998) resolved this issue presenting a trial design and analysis that permitted estimations of both vaccine efficacies without the need of contact data. This design required at least two populations to be vaccinated with different vaccination coverage. Following on this approach, the Kilkenny vaccine trial consisted of three badger populations vaccinated with: 0,50 and $100 \%$ vaccine coverage. This trial differed from other human trials in the fact that the required vaccination coverages were achieved over time rather than instantaneously, and this is something that was taken into account in our design and analysis. 
Chapter 3: Estimating the power of a Mycobacterium bovis vaccine trial in Irish badgers

When designing a (vaccine) trial, it is of great importance to ensure that the design will enable, to a specified level, often set to $80 \%$, an effect to be found (if the effect really exists). In other words, it is critical that the statistical power of the study is sufficient (Riggs and Koopman, 2004, 2005). In many studies, power calculations are done a priori in order to determine the minimum/optimal sample size. In the Kilkenny trial, sample size was somehow pre-determined by logistical issues, as it was not possible to enlarge the trial area. Nonetheless, it was important that other factors that could affect power were examined. In the third chapter of the thesis, we carried out a study of factors that could affect our ability to detect a positive effect of vaccination if present, including: sample size (recapture percentage), initial prevalence, sensitivity and specificity of the diagnostic test used, transmission rate between unvaccinated badgers, and assumed $\mathrm{VE}_{\mathrm{S}}$ and $\mathrm{VE}_{\mathrm{I}}$.

Chapter 4: Optimising and evaluating the characteristics of a multiple antigen ELISA for detection of $M$. bovis infection in a badger vaccine field trial

The results from Chapter 3 showed that the factor with the greatest potential to compromise our ability to detect a positive effect of the BCG vaccine was the specificity of the diagnostic test used to determine infection in badgers. A diagnostic test with a nearly perfect specificity $(>99.8 \%)$ was necessary to achieve a study power of at least $60 \%$. As a diagnostic test with such a large specificity was not commercially available, the Enfer test to be used in the Kilkenny vaccine trial was optimized and evaluated.

Chapter 5: Quantification of Mycobacterium bovis transmission in a badger vaccine field trial

Using the optimized Enfer test, $\mathrm{VE}_{\mathrm{S}}$ and $\mathrm{VE}_{\mathrm{I}}$ were calculated from the trial carried out in Kilkenny (2009-2013) and results are presented in Chapter 5. The impact of vaccination on transmission between badgers was evaluated assuming badgers are the only host for M. bovis. Nonetheless, ultimately, it was the effect of badger vaccination on transmission in the cattle-badger system that was of interest.

Chapter 6: Characterizing transmission in a Mycobacterium bovis multi-host system

If an infectious agent is transmitted between two (or more) different host species, $\mathrm{R}_{0}$ calculations become more complex. These systems can be modelled using the Next Generation Matrix (NGM) (Diekmann et al., 1990, 2010; Fulford et al., 2002) and $\mathrm{R}_{0}$ can be determined mathematically as the dominant eigenvalue of this NGM. The 
challenge in estimating $\mathrm{R}_{0}$ in this way arises from the need to parameterise these models with data carrying large uncertainty. In Chapter 6 , a solution to this problem is presented: the transition matrices for cattle herds and badgers at the endemic (M) and disease free (NGM) equilibrium were estimated by solving M.e $=\mathrm{e}$ and calculating the NGM from $M$. These equations did not require data on the transmission parameters between species, i.e from badgers to cattle and vice versa, in this way avoiding the introduction of large uncertainty.

\section{Chapter 7: General Discussion}

Finally, the results of this thesis are placed and analysed in a broader context so they can best inform policy in this area. The challenges of introducing badger vaccination in the bTB strategy are discussed. Suggestions on the road map to eradication are made and further research is suggested.

\section{References}

Aznar, I., McGrath, G., Murphy, D., Corner L.A.L., Gormley, E., Frankena, K., More, S.J., Martin, W., O’Keeffe, J., de Jong M.C.M., 2011. Trial design to estimate the effect of vaccination on tuberculosis incidence in badgers. Veterinary Microbiology 151, 104-111.

Convention on the Conservation of European Wildlife and Natural Habitats. Standing Committee, 1997. Texts adopted by the Standing Committee of the Bern Convention on the Conservation of European Wildlife and Natural Habitats (19 September 1979):1982-96. Strasbourg :Council of Europe Publishing.

Costello, E., Flynn, O., Griffin, J., Quigley, F., O’Grady, D., McGrath, G., Hammond, R.F., 1999. Spatial distribution of RFLP types identified in Mycobacterium bovis isolates from badgers and cattle in a study area in the Republic of Ireland. In: Collins, J.D., Hammond, R.F. (Eds.), Selected Papers 1999. University College Dublin, pp. 26-30.

Corner, L.A.L., O’Meara, D., Costello, E., Gormley, E., 2005. Tuberculosis in badgers: true prevalence, diagnostic methods and epidemiology (poster). Society for Veterinary Epidemiology and Preventive Medicine, Nairn, Scotland.

Corner, L.A.L., Costello, E., Lesellier, E., O’Meara, D., Sleeman, D.P., Gormley, E., 2007. Experimental tuberculosis in the European badger (Meles meles) after endobronchial inoculation of Mycobacterium bovis: I. Pathology and bacteriology. Research in Veterinary Science 83, 53-62.

Corner, L.A.L., Costello, E., Lesellier, S., O’Meara, D., Gormley, E., 2008. Vaccination of European badgers (Meles meles) with BCG by the subcutaneous and mucosal routes induces protective immunity against endobronchial challenge with Mycobacterium bovis. Tuberculosis 88, 601-609. 
Diekmann, O., Heesterbeek, J.A.P. and Metz, J.A.J., 1990. On the definition and computation of the basic reproduction ratio $\mathrm{R}(0)$ in models for infectious diseases in heterogeneous populations. Journal of Mathematical Biology 28, 365-382.

Diekmann, O., Heesterbeek J.A.P. and Roberts M.G., 2010. The construction of next generation matrices for compartmental epidemic models. Journal of the Royal Society Interface 7, 873-885.

Eves, J.A., 1999. Impact of badger removal on bovine tuberculosis in east County Offaly. Irish Veterinary Journal 52, 199-203.

Fulford, G.R., Roberts, M.G., Heesterbeek, J.A.P., 2002. The metapopulation dynamics of an infectious disease: tuberculosis in possums. Theoretical Population Biology 61, 15-29.

Gormley, E., Costello, E., 2003 Tuberculosis and badgers: new approaches to diagnosis and control. Journal of Applied Microbiology 94, 80-86.

Griffin, J.M., Williams, D.H., Kelly, G.E., Clegg, T.A., O’Boyle, I., Collins, J.D., More, S.J., 2005. The impact of badger removal on the control of tuberculosis in cattle herds in Ireland. Preventive Veterinary Medicine 67, 237-266.

Halloran, M.E., Struchiner, C.J., Longini Jr., I.M., 1997. Study designs for evaluating different efficacy and effectiveness aspects of vaccines. American Journal of Epidemiology 146, 789-803.

Halloran, M.E, Longini Jr., I.M., Struchiner, C.J., 1999. Design and interpretation of vaccine field studies. Epidemiological Reviews 21, 73-88.

Kermack, W.O. and McKendrick, A.G., 1927. Contributions to the mathematical theory of epidemics. 1. Reprinted from Proceedings of the Royals Society 115 (A), 700-721.

Krebs, J.R., Anderson, R.A., Clutton-Brock, T., Morrison, I., Young, D., Donnelly, C., 1997. Bovine tuberculosis in cattle and badgers. Report to the Rt. Hon Dr. Jack Cunningham MP. London: MAFF Publications.

Lesellier, S., Corner, L., Costello, E., Lyashchenko, K., Greenwald, R., Esfandiari, J., Singh, M., Hewinson, R.G., Chambers, M., Gormley, E., 2009. Immunological responses and protective immunity in BCG vaccinated badgers following endobronchial infection with Mycobacterium bovis. Vaccine 27, 402-409.

Lesellier, S., Palmer, S., Gowtage-Sequiera, S., Ashford, R., Dalley, D., Davé, D., Weyer, U., Salguero, F.J., Nunez, A., Crawshaw, T., Corner, L.A.L., Hewinson, R.G., Chambers, M.A., 2011. Protection of Eurasian badger (Meles meles) from tuberculosis after intra-muscular vaccination with different doses of BCG. Vaccine 29, 3782-3790.

Longini Jr., I.M., Sagatelian, K., Rida, W.N., Halloran, M.E., 1998. Optimal vaccine trial design when estimating vaccine efficacy for susceptibility and infectiousness from multiple populations. Statistics in Medicine 17, 1121-1136.

More, S.J., 2005. Towards eradication of bovine tuberculosis in Ireland: a critical review of progress. In: Proceedings, 2005 Annual Meeting Society for Veterinary 
Epidemiology and Preventive Medicine, Nairn, Scotland. Edited by D. J. Mellor, A. M. Russell and J. L.N. Wood. pp 13-23.

More, S.J., Good, M., 2006. The tuberculosis eradication programme in Ireland: a review of scientific and policy advances since 1988. Veterinary Microbiology 112, 239-251.

More, S.J., Collins, J.D., Good, M., Skuce, R.A., Pollock J.M. and Gormley, E., 2006. The tuberculosis eradication programme in Ireland: A review of the scientific and policy advances since 1988. In: Proceedings of the Fourth International Conference on Mycobacterium bovis. Veterinary Microbiology $112,239-251$.

More, S.J., 2009. What is needed to eradicate bovine tuberculosis successfully: An Ireland perspective. Veterinary journal, 180 (3), 275-8.

Murphy, D., Costello, E., Aldwell, F.E., Lesellier, S., Chambers, M.A., Fitzsimons, T., Corner, L.A.L., Gormley, E., 2014. Oral vaccination of badgers (Meles meles) against tuberculosis: Comparison of the protection generated by BCG vaccine strains Pasteur and Danish. Veterinary Journal 200, 362-367.

Noonan, N.L., Sheane, W.D., Harper, W.R., Ryan, P.J., 1975. Wildlife as a possible reservoir of bovine tuberculosis. Irish Veterinary Journal 29.

Olea-Popelka, F.J., Fitzgerald, P., White, P., McGrath, G., Collins, J.D., O'Keeffe, J., Kelton, D.F., Berke, O., More, S., Martin, S.W., 2009. Targeted badger removal and the subsequent risk of bovine tuberculosis in cattle herds in county Laois, Ireland. Preventive Veterinary Medicine 88, 178-184.

Palmer, M.V., 2013. Mycobacterium bovis: characteristics of wildlife reservoir hosts. Transboundary and Emerging Diseases 60, 1-13.

Riggs, T.W., Koopman, J.S., 2004. A stochastic model of vaccine trials for endemic infections using group randomization. Epidemiology and Infection 132, 927-938.

Riggs, T., Koopman, J.S., 2005. Maximizing statistical power in group randomized vaccine trials. Epidemiology and Infection 133, 993-1008.

Watchorn, R.C.(1965). Bovine Tuberculosis Eradication Scheme 1954-1965. Dublin: Department of Agriculture and Fisheries.

Wirth, T., Hildebrand, F., Allix-Béguec, C., Wolbeling, F., Kubica, T., et al., 2008. Origin, spread and demography of the Mycobacterium tuberculosis complex. PLoS Pathogens 4, e1000160. doi:10.1371/journal.ppat.1000160 7 

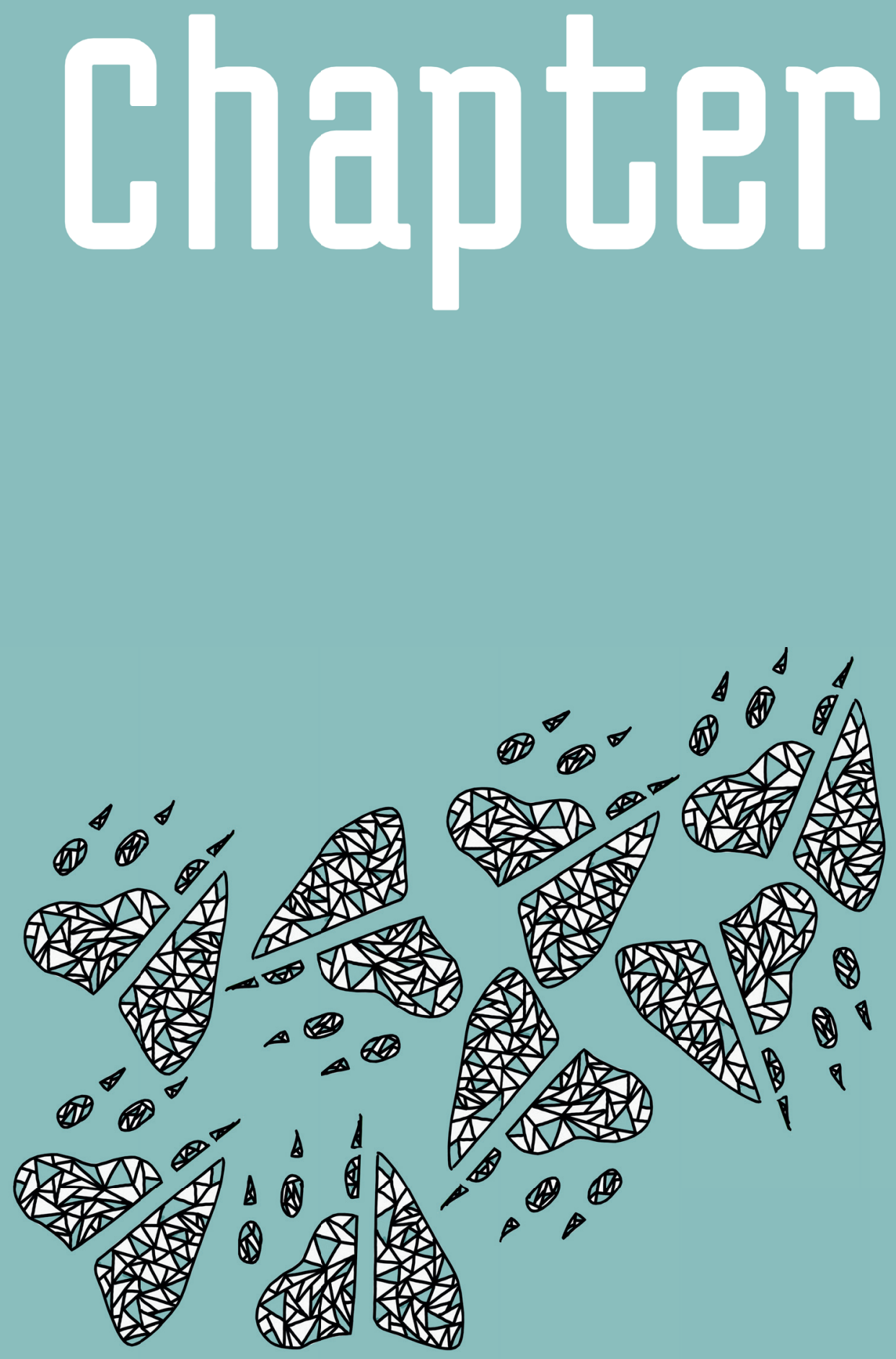


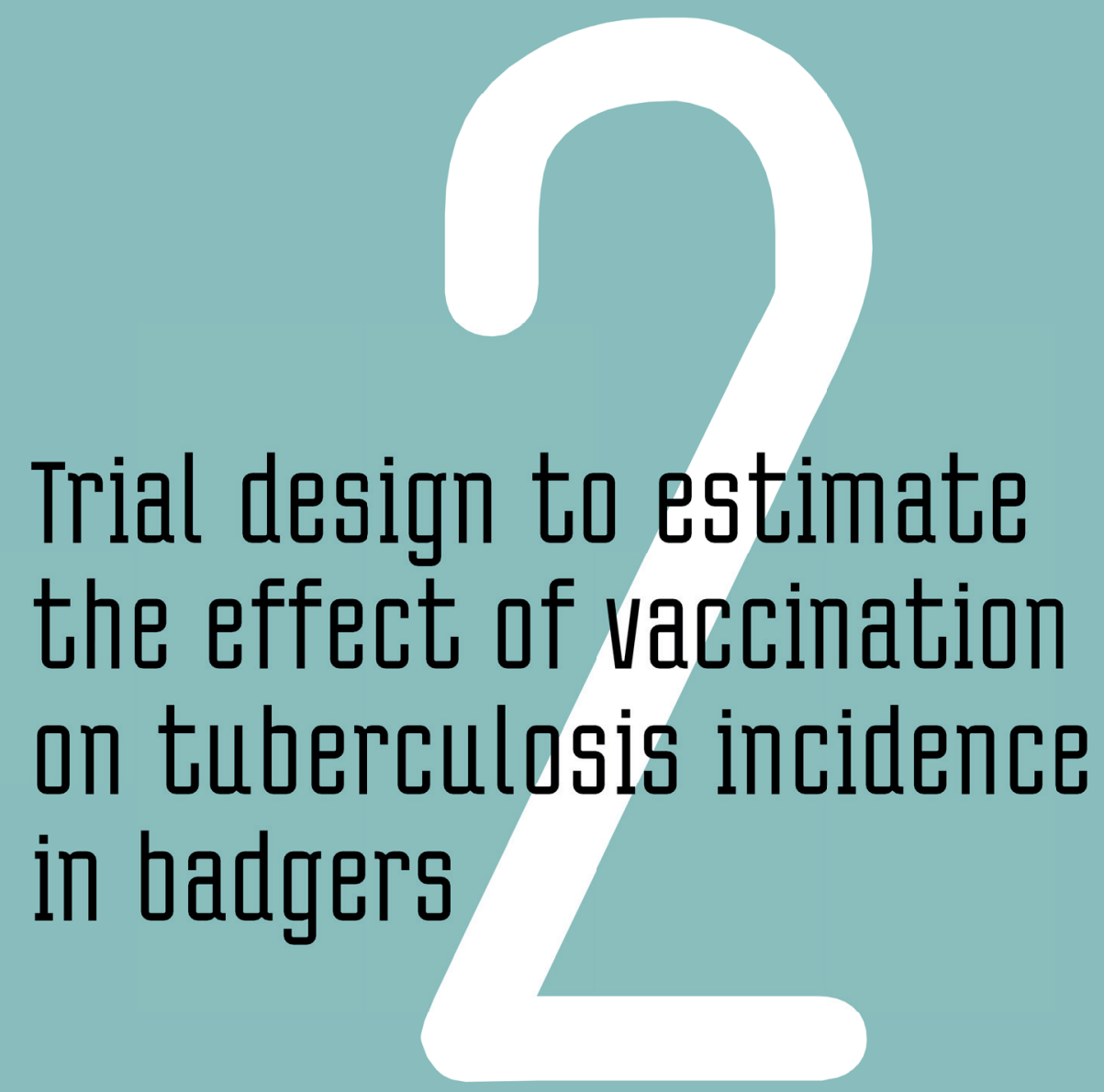

Aznar, I. , McGrath, G. , Murphy, D. , Corner, L.A.L., Gormley, \&. , Frankena, K. , More, 5.J. , Martin, W. , O'Keeffe, J., de Jong, M.C.M. 
Chapter 2

\title{
Trial design to estimate the effect of vaccination on tuberculosis incidence in badgers
}

\begin{abstract}
Aznar, I., McGrath, G., Murphy, D., Corner, L.A.L., Gormley, E., Frankena, K., More, S.J., Martin, W., O’Keeffe, J., de Jong, M.C.M.
\end{abstract}

Veterinary Microbiology (2011) 151, 104-111

(C) 2011 Elsevier B.V. All rights reserved. 


\begin{abstract}
The principal wildlife reservoir of Mycobacterium bovis in Ireland is the European badger. Studies in the Republic of Ireland (RoI) have shown that badgers culled in association with cattle herd tuberculosis breakdowns (focal culling) have a higher prevalence of infection than the badger population at large. This observation is one rationale for the medium term national strategy of focal badger culling. A vaccination strategy for the control of bovine tuberculosis (bTB) in badgers is a preferred long-term option. The Bacillus Calmette-Guérin (BCG) vaccine has been shown to decrease disease severity in captive badgers under controlled conditions. As the vaccine has been tested in a controlled environment with precise information on infection pressure, it cannot be assumed a priori that the effects of vaccination are similar in the wild, where other environmental and/or ecological factors prevail. For this reason we have designed a vaccine field trial to assess the impact of vaccination on the incidence of TB infection in a wild badger population.
\end{abstract}

The selected study area for the vaccine trial (approximately 755 square kilometers) is divided into three zones each of which has similar characteristics in terms of size, number of main badger setts, cattle herds, cattle and land classification type. Three vaccination levels $(100 \%, 50 \%$ and $0 \%)$ will be allocated to the three zones in a way that a gradient of vaccination coverage North to South is achieved. The middle zone (Zone B) will be vaccinated at a $50 \%$ coverage but Zone $\mathrm{A}$ and $\mathrm{C}$ will be randomly allocated with $100 \%$ or $0 \%$ vaccination coverage. Vaccination within Zone B will be done randomly at individual badger level.

The objective of this paper is to describe the design of a field TB vaccination trial for badgers, the epidemiological methods that were used to design the trial and the subsequent data analysis. The analysis will enable us to quantify the magnitude of the observed vaccination effect on $M$. bovis transmission in badgers under field conditions and to improve our knowledge of the biological effects of vaccination on susceptibility and infectiousness. 


\section{Introduction}

Ireland initiated an eradication program for bovine tuberculosis (bTB) as early as 1950 (More and Good, 2006). The adopted test-and-slaughter policy achieved a 97\% reduction in cattle tuberculosis prevalence, from $17 \%$ to $0.5 \%$ prevalence in the initial ten years (Watchorn, 1965). Since then, bTB prevalence has remained relatively unchanged despite the introduction of a range of measures aimed at reducing cattle to cattle transmission (Griffin and Dolan, 1995).

In the 1970s in England, badgers were first suspected as a reservoir for Mycobacterium bovis (Krebs, 1997). The first infected badger was discovered in Ireland in 1974 (Noonan et al., 1975). Since then numerous papers have been published that confirm badgers as the main wildlife M. bovis reservoir in Ireland and England (Barrow and Gallagher, 1981; Cheeseman et al., 1981; Fagan, 1993; Gallagher et al., 1998). A recent study carried out in Ireland detected a prevalence of 36.3\% in badgers trapped as part of Department of Agriculture, Fisheries and Food (DAFF) culling operations; the prevalence reported here was much higher than in previous studies where less comprehensive bacteriological culture methods had been used (Murphy et al., 2010).

The precise role of badgers in the dynamics of bTB is not clear. Several studies in Ireland have linked badger removal with a subsequent reduction in bTB incidence (Eves, 1999; Griffin et al., 2005; More and Good, 2006; Olea-Popelka et al., 2009). However, in a field trial carried out in Britain, the reduction in cattle TB incidence in culled areas was only modest and an increase in TB incidence, albeit transient (Jenkins et al., 2008), was observed in non culled neighboring areas (Woodroffe et al., 2007). Pope et al. (2007) concluded that the increased prevalence observed in neighboring areas was associated with medium and long-distance badger dispersal and emphasized the importance of taking into account the potential negative effects associated with badger dispersal when using culling as a disease control strategy. Although there are discrepancies between different studies about the efficiency of badger culling in the control of bTB, they all provide evidence that badgers play an important role in the maintenance of it. Therefore, addressing infection in badgers is considered vital when trying to control bovine tuberculosis in the aforementioned countries. In the short- to medium-term in Ireland, focused badger culling is being conducted as part of a broader national TB control programme, to limit TB maintenance in areas where TB incidence in cattle is high. However, badgers are a protective species in the Republic of Ireland (ROI) under the 1976 Wildlife Act. Consequently, alternative strategies to badger culling are being sought in the long term for the control of bovine tuberculosis. 
In 2001, a 10-year work program was designed in Ireland to study the possibility of using Bacillus Calmette-Guérin (BCG) vaccine in badgers to assist in the control and eradication of TB in cattle. The program consisted of a sequence of experimental studies carried out initially with captive badgers (Corner et al., 2007; Lesellier et al., 2009). Although vaccines can be tested in a controlled environment for evidence of both protection and decreased transmission, it cannot be assumed a priori that the effects of vaccination are similar in the wild where other factors may play a role. For this reason, a vaccine field trial to assess the impact of vaccination on the incidence of tuberculosis in a wild badger population has been designed as part of the 10-year project.

The objective of this paper is to describe a field trial design, the epidemiological methods that were used to design the trial and the subsequent data analysis. The analysis will enable us to quantify the magnitude of the observed vaccination effect on transmission under field conditions and to improve our knowledge of the biological effects of the vaccination on susceptibility and infectiousness of badgers.

\section{Theoretical basis: a review}

\subsection{Initial considerations in designing a vaccine trial}

Specifying clear question(s) of interest is essential when designing a trial to evaluate the effects of vaccination. Different vaccination programs have different aims; the question of interest could vary, for example from how good vaccination is in protecting the individual against infection to what reduction in infectiousness can be achieved. The effect of interest will determine the study unit, parameters of effect, as well as the level of information required (Halloran et al., 1997). The main question of interest in our study is to determine the efficiency of badger vaccination in reducing $M$. bovis transmission. Specifically we are interested in the value of the reproduction ratio $(R)$ at different levels of vaccination $(p)$; ie $R(p)$. We will clarify how to estimate $R(p)$ subsequently.

Vaccines that reduce transmission in a population can have a beneficial effect in vaccinated as well as in unvaccinated individuals. Most studies of vaccine efficacy emphasize the direct benefit of vaccination (often called 'protective ability') to vaccinated individuals. Following Halloran et al. (1999), we denote this as $\mathrm{VE}_{\mathrm{S}}$ or vaccine efficacy for susceptibility. In addition, there often are indirect benefits for both vaccinated and unvaccinated individuals which Halloran et al. denoted as vaccine efficacy for infectiousness $\left(\mathrm{VE}_{\mathrm{I}}\right)$. These measures will be estimated in this trial; however, for subsequent modelling purposes we will emphasize $R(p)$ 


\subsection{Parameters of effect}

In order to estimate $R(p)$, we define four transmission parameters:

- transmission from a vaccinated to a vaccinated badger $\left(\beta_{V V}\right)$;

- transmission from a vaccinated to an unvaccinated badger $\left(\beta_{V U}\right)$;

- transmission from an unvaccinated to a vaccinated $\left(\beta_{U V}\right)$, and

- transmission from an unvaccinated to an unvaccinated badger $\left(\beta_{U U}\right)$.

The average number of secondary cases caused by one typically infected individual in a fully susceptible population (Diekmann and Heesterbeek 2000) is called the basic reproduction ratio $\left(R_{0}\right)$. Reproduction ratios of host populations in which interventions are taking place are often represented by $R$ to distinguish them from $R_{0}$. The reproduction ratio as a function of the proportion of vaccinated badgers $R(p)$ can be determined from the transmission rates described above and the time that infected badgers remain infectious $(T)$. Transmission rates are a combination of the infectiousness of the donor and the susceptibility of recipient individuals, and since vaccination with BCG has the potential to affect both of these, $R(p)$ will be an important parameter for understanding the impact of badger vaccination in disease transmission and population dynamics of $M$. bovis. Ultimately, the $R(p)$ obtained from the badger vaccine trial will be used in further modelling aimed at assessing badger vaccination as a strategy to control/eradicate $M$. bovis infection in cattle.

As noted above, vaccine efficacy/effectiveness has been traditionally defined as 1 minus some measure of relative risk (RR) of the incidence of disease in the vaccinated group compared to the incidence in the non-vaccinated group (Halloran et al., 1999). In the past, the main objective of human vaccine studies was to measure individual protection against infection or disease $\left(\mathrm{VE}_{\mathrm{S}}\right)$. Perhaps less appreciated, but not less important, is the ability of a vaccine to reduce the duration or severity of the infectiousness of those vaccinates that become infected ( $\left.\mathrm{VE}_{\mathrm{I}}\right)$ (Longini et al, 1998). In recent experiments, M. bovis was recovered from both vaccinated and nonvaccinated badgers after being challenged with the mycobacterium; however, a reduction in the size, number and distribution of gross and histological lesions in vaccinated badgers compared to non-vaccinated badgers was demonstrated (Corner et al., 2008). Vaccination did not confer individual protection against infection in the mentioned study, but this has to be cautiously interpreted as vaccine protection could be dependent on the infection dose. It is not known what the infectious dose is in natural infections. In field vaccine trials in possum in New Zealand, using conjunctival and intranasal vaccination (Corner et al., 2002) and more recently vaccination by oral delivery (Tompkins et al., 2009), significant protection against natural exposure was seen in the vaccinated group compared to the control groups. Protection was much higher than predicted from previous studies where possums had been experimentally challenged (Corner et al., 2001; Buddle et al., 2006). 
Estimation of $R(p), \mathrm{VE}_{\mathrm{S}}$ and $\mathrm{VE}_{\mathrm{I}}$ will give us a more detailed understanding of the ways $B C G$ vaccine works in a wild badger population.

\section{Epidemiological contribution to the design of the vaccine trial}

\subsection{Study site}

Prior to deciding on our study site for the badger vaccine trial, several epidemiological and logistic factors were considered:

- Culling history. Because of the badger culling pollicy in Ireland, it was important to have confidence that the area selected for the trial had been protected from culling for some time before the commencement of the trial. Badger culling in that area could have had a negative effect not just in the total number of individuals captured but also on the initial prevalence of TB among badgers.

- Knowledge of sett location. Knowledge of the area in terms of sett location was considered an advantage and helped in dividing the study area into three similar zones (see below).

- Community and technical support. Good support from the local farming community as well as from from both the District Veterinary Offices (DVOs) and Regional Veterinary Laboratories (RVLs) was vital.

Based on these criteria, the area selected for the vaccine trial is located in County Kilkenny (Fig. 1). 


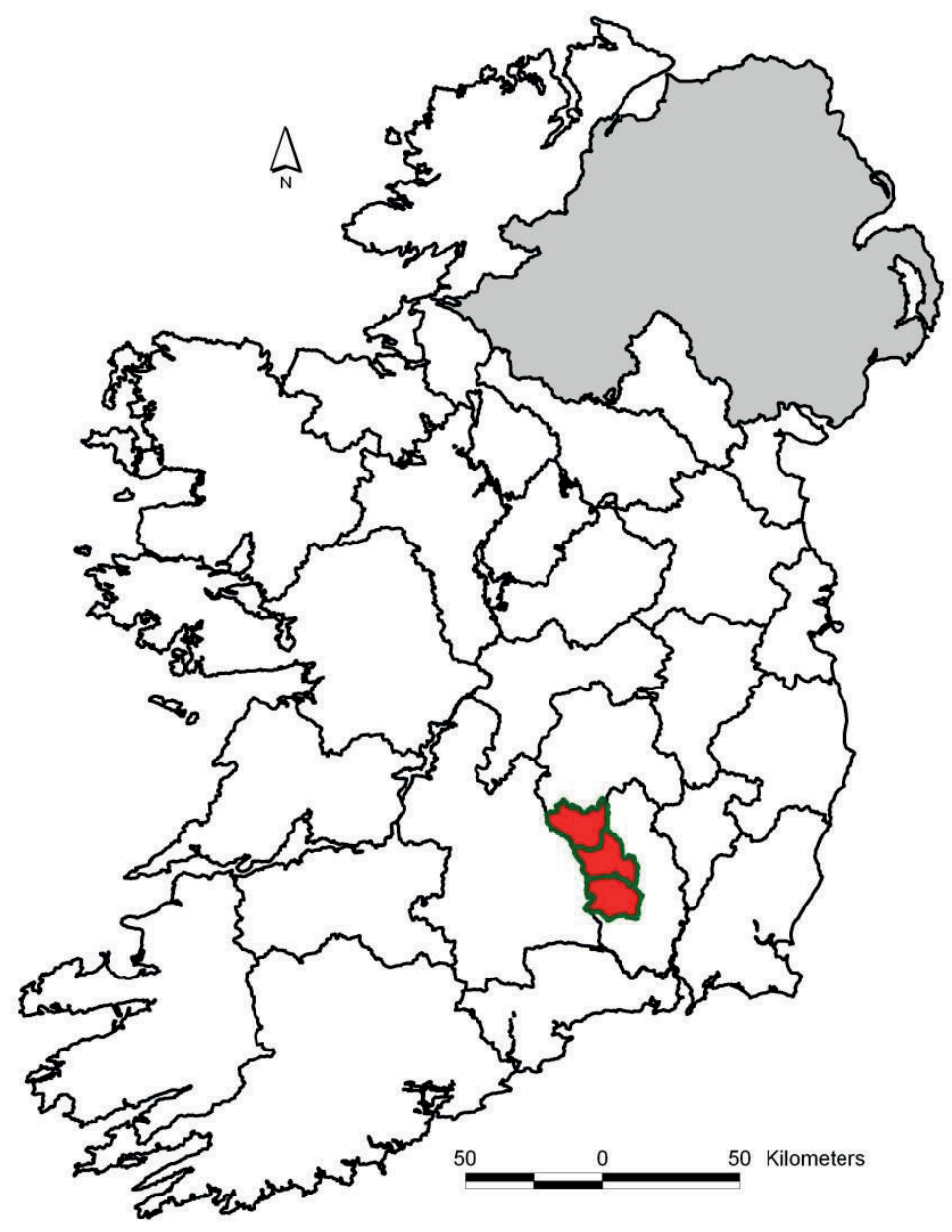

Fig. 1. Study site selected for the vaccine trial is highlighted in red.

The size of the area is approximately 755 square kilometers. This area had been part of one of the reference areas in the Four Area Project (FAP) (Griffin et al., 2005) and will have been protected from culling for at least two years before the start of the vaccine trial. A prevalence of infection of $30 \%$ is expected based on historical data from neighbouring areas.

\subsection{Trial design}

A vaccine trial to exclusively determine $\mathrm{VE}_{\mathrm{S}}$ can be designed by vaccinating one or several populations with the same vaccination coverage, with coverage being strictly 
inferior to $100 \%(40 \%, 50 \%, 60 \%$, etc. $)$ so that a number of infections within the vaccinated group can occur. Because we aimed to estimate both $\mathrm{VE}_{\mathrm{S}}$ and $\mathrm{VE}_{\mathrm{I}}$, then two populations vaccinated with different vaccination coverage are required (Longini et al., 1998). In this paper it is explained how the vaccination fractions for these two populations have to be selected to maximise the differences on indirect effects between the two populations. This can be achieved with one population vaccinated at $0 \%$ and the other vaccinated at the highest percentage that allows a minimum number of infections to happen (critical vaccination fraction). Likewise to estimate the four betas (transmission rates), different levels of coverage are needed (DeJong et al., 1996).

Our objectives could have therefore been attained with two populations, one vaccinated at $0 \%$ and the other at $100 \%$ vaccination coverage (badger trapping rates will never be $100 \%$ effective; also the dynamics of the badger population ensures a certain number of susceptible badgers every year and therefore a certain number of infections). We chose though to include a third population vaccinated at $50 \%$ to optimise the design in two ways: firstly by making sure that there will be enough new infections even if the $100 \%$ vaccination would lead to $R<1$ and secondly by allowing us to estimate the area effect (in the case of only two populations, the parameters would be estimated from different populations and it would not be possible to disentangle the area effect).

The trial area is divided into three zones with similar characteristics in terms of size, number of main badger setts, cattle herds, cattle and land classification (Fig. 2). In these zones, vaccination coverage will be 100,50 and $0 \%$, with the gradient of coverage (either $100 \%$ to $0 \%$ from north to south, or vice-versa) being allocated randomly at the start of the trial. Badger data were acquired through rigorous surveying of the entire study area. Previous surveying had been performed in sections of the study area as part of the FAP reference area and through the activities of the DAFF's licensed badger culling policy on setts adjacent to herd bTB breakdowns. Setts previously recorded were revisited during the recent survey and assessed for signs of activity. All bovine data were derived from the Animal Health Computer System (AHCS) and farm outlines were taken from the Land Parcel Identification System (LPIS). Land use classifications were defined using the CORINE dataset (Coordination of Information on the Environment, 2000). Using ArcMap 9.2 (ESRI, Redlands, CA, USA) with geo-rectified colour orthophotography and vectorized 1:50,000 data (Ordnance Survey Ireland, Dublin, Ireland), natural boundaries, where possible, were selected to define the perimeter and internal boundaries of the study area. The aim was to achieve similar infection pressure from cattle and badgers in the three zones. 


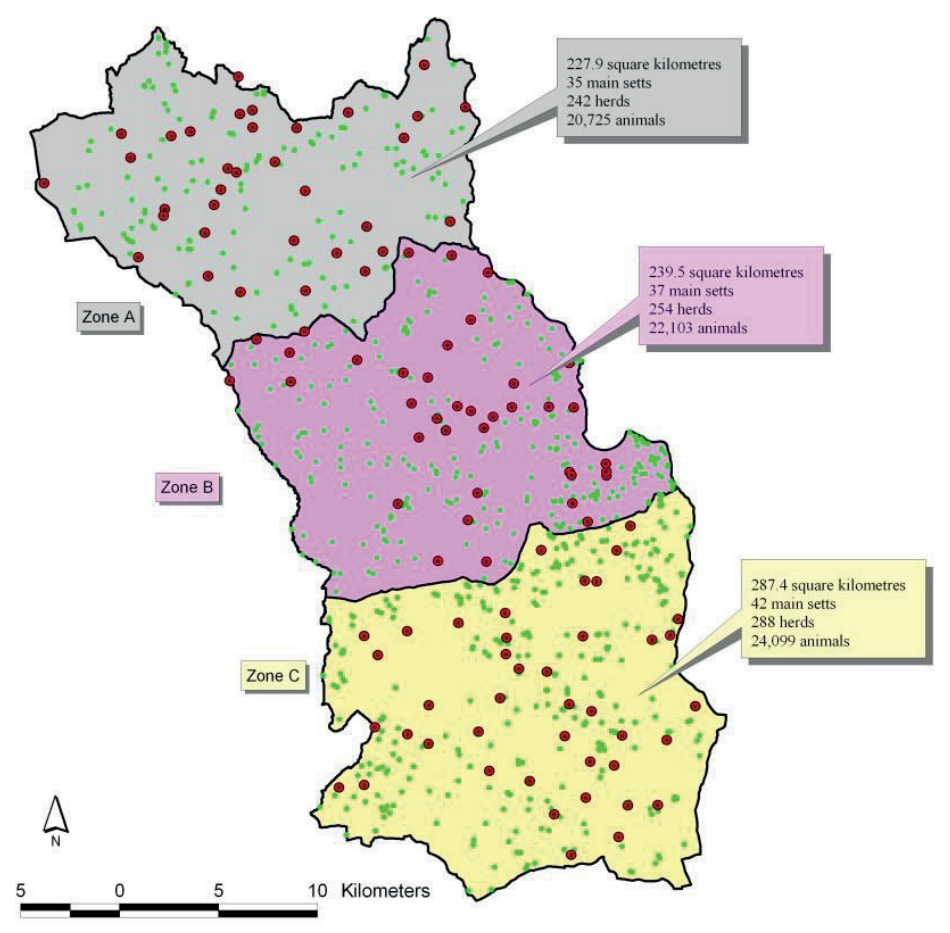

Fig. 2. Map showing study area divided into three zones A, B and C (grey, pink and yellow, respectively) where vaccination coverage will be 100,50 and $0 \%$. The gradient of coverage ( $100 \%$ to $0 \%$ from north to south, or vice-versa) will be allocated randomly at the start of the trial. Main badger setts are represented with red dots, and green dots represent other sett types.

Badgers will be captured, treated with a vaccine or a placebo depending on the randomly allocated treatment and then released. In zone B (with 50\% vaccine coverage), each badger at first capture will be randomly allocated to either vaccine or placebo. In each zone throughout the study period, the treatment will be administered every year to avoid possible waning of vaccine effects. Live M. bovis BCG strain Danish will be used. It will be prepared in a lipid formulation for oral delivery, containing $10^{8}$ colony forming units $/ \mathrm{ml}$. A lipid-only placebo with identical visual characteristics, texture and viscosity to the vaccine, and in identical syringes, will also be used (Aldwell et al., 2003a,b). Vaccine and placebo control samples will 
be coded at the laboratory where they are prepared, and neither field staff nor data analysts will be aware of the vaccine status of individual animals.

The trial will employ a capture-tag-release regime with both cages and stopped wire restraints being used. Badgers in the trial will be 'hand vaccinated'; that is, each animal will be individually orally dosed with the vaccine. Each badger will be permanently identified with a tattoo and passive transponder (microchip) when first captured. At each capture, badgers will be examined and a blood sample collected. Humoral immune responses (serologic titres) will be used to determine the badger's infection status and to detect a change in infection status; that is, to detect both preexisting infection in badgers as they are recruited to the study and the occurrence of new infections on recapture. Key data, including sex, estimated age (cub, juvenile, yearling, adult and old adult), body weight, presence of injuries and the GPS location of the cage trap or restraint, will be recorded at each badger capture. All data collected in the field will be recorded onto handheld computers. The trial will last four years and there will be two 'catching' sweeps of the entire area each year. At the end of the trial, badgers in the three zones will be depopulated and a detailed post mortem examination will be conducted on all badgers, involving an examination for gross pathology and the collection of samples for histopathology and bacteriology. The severity of infection will be assessed from the number, distribution and the severity of gross lesions, the number and distribution of histological lesions, and the number and distribution of culture positive tissues and the bacterial load in those tissues.

Badger removal will be undertaken within the trial area when three or more standard reactors are disclosed in a herd only if active badger setts are found in the farm and all other sources of infection (residual, purchase and farm to farm spread) have been ruled out by an epidemiological investigation. If culling of badgers is deemed necessary for control of tuberculosis in cattle herds, the culling will be carried out by field staff of the project when they next trap in the designated area.

\section{Analysis of the vaccine trial data}

\subsection{General description}

The outcome from the vaccine trial will be in the form of a Bernoulli experiment: as badgers are trapped and a blood sample taken, we will gather information on whether these badgers have become or are infected (assigned value 1) or not (assigned value 0 ) during the time at risk (time between two subsequent trappings). Infection in this case will be defined by serologic results i.e. by sero-positivity. Records on individual badgers will be taken at each successful capture (not necessarily at each trapping 
exercise) such as location of the badger at the time of the trapping (zone A, B or C) and its vaccination status. Other demographic data will be also recorded.

From the observations at each subsequent capture of each individual badger, the following variables will be extracted:

- Time interval $(\Delta \mathrm{t})$ between the two captures

- Disease status of the badger at the beginning and at the end of the time interval

- Vaccination status of the badger

- Zone where the animal was observed at the beginning and end of the time interval (A, B or C)

- Average prevalence of infection in the zone where the badger was caught during $\Delta \mathrm{t}$ (PrevA, PrevB, PrevC)

- Average fraction of infected badgers that are vaccinated during $\Delta t$ in the zone where the badger was caught $\left(\mathrm{F}_{\mathrm{VA}}, \mathrm{F}_{\mathrm{VB}}, \mathrm{F}_{\mathrm{VC}}\right)$

The rate at which vaccinated/non-vaccinated badgers acquire infection from vaccinated and non-vaccinated badgers $\left(\beta_{V V}, \beta_{V U}, \beta_{U V}, \beta_{U U}\right)$ can be estimated based on the observed probability of becoming infected for each of these individuals. The estimated $\beta \mathrm{S}$ will be used to calculate $\mathrm{VE}_{\mathrm{S}}, \mathrm{VE}_{\mathrm{I}}$ and the reproduction ratio as a function of the vaccination coverage $(R(p))$.

\subsection{Estimation of the transmission parameters}

The rate at which vaccinated/non-vaccinated badgers acquire infection from vaccinated and non-vaccinated badgers $\left(\beta_{V V}, \beta_{V U}, \beta_{U V}, \beta_{U U}\right)$ can be estimated based on the observed probability of becoming infected for each of these individuals. For the purposes of explanation, we shall at first ignore the vaccination state of the badgers. A stochastic susceptible-infectious (SI) model can be used then to describe the transmission of $M$. bovis in the trial where transmission can occur if an infectious and a susceptible individual make contact. The number of infectious contacts (NIC, contacts with an infectious individual) encountered by each randomly chosen susceptible individual in a period of time $\Delta t$ can be expressed as:

$$
N I C=\beta *(I / N) * \Delta t
$$

Where $\beta$ is the transmission parameter, $\Delta t=$ time interval, $\mathrm{I}=$ number of infected individuals*, $\mathrm{N}=$ total number of individuals, $\mathrm{I} / \mathrm{N}=$ prevalence of infected badgers*

* For our purpose infected badgers are deemed to be infectious.

From the above it can be derived that the number of 'successful' infectious contacts (infectious contacts that result in transmission events) encountered by this randomly 
chosen individual in a period of time $\Delta t$ follows a Bernoulli distribution with probability:

$$
p=1-e^{-\beta * I * \Delta t / N}
$$

The expected number of new infections $(E(C))$ among all susceptible badgers at the end of the time interval between two captures can then be modelled using a binomial distribution where $\mathrm{S}$ is the number of susceptible badgers at the beginning of the time interval and the probability that each of these badgers will become infected during that time interval is defined by $1-e^{-\beta * I * \Delta t / N}$,

$$
E(C)=S *\left(1-e^{-\beta * I * \Delta t / N}\right)
$$

Based on serologic results we will be able to determine the number of new cases among the susceptible badgers (C). By using a generalized linear model (GLM) with a complementary-log-log link function, a binomial error function, with binomial total $\mathrm{S}$ and offset $\ln ((I / N) * \Delta t)$, the transmission parameter $\beta$ can be estimated.

Following the introduction of the vaccination protocol, there will be heterogeneity in the vaccination status of badgers; we will have four different $\beta$ values with two sub indexes, of the type $\beta_{v u}$. The first sub index will refer to the vaccination state of the infectious badger and the second sub index to the vaccination state of the susceptible animal (to which the first has made contact). Clearly, we know the vaccination status of the receiving susceptible badger and thus we can calculate $\beta . u$ and $\beta_{. v}$ separately. However to account for donor (infectious) badger, we use the differences in the fraction of infected badgers that are vaccinated in the different zones.

Let's assume that a badger that was originally trapped in zone A at time ' $\mathrm{t}$ ' is trapped again in zone $\mathrm{A}$ at time ' $\mathrm{t}+1$ ' and that this badger had been vaccinated. Vaccination coverage in zone A is targeted to be $100 \%$, but since not all badgers will be trapped in each trapping exercise it will take 2-3 trapping rounds to approach this goal. Our hypothetical badger will then have infection pressure coming from both vaccinated and non-vaccinated infected badgers. The number of infectious contacts encountered by this randomly selected badger in that period of time is defined by:

$$
N I C=\left(\beta_{V V} * I_{V} / N+\beta_{U V} * I_{U} / N\right) * \Delta t
$$


If we assume that there is a multiplicative effect on NIC as the fraction of infected vaccinated badgers increases, after doing some algebraic manipulations (De Jong et al., 1996):

$$
N I C=e^{\beta_{V V} * F_{V A}+\beta_{U V} *\left(1-F_{V A}\right)} * \operatorname{Prev} A * \Delta t
$$

or:

$$
N I C=e^{\beta_{U V}+\left(\beta_{V V}-\beta_{U V}\right) * F_{V A}} * \operatorname{Prev} A * \Delta t
$$

where $\mathrm{F}_{\mathrm{VA}}$ is the fraction of infected badgers that are vaccinated in zone $\mathrm{A}$ and PrevA is the average prevalence of infection in zone A during $\Delta \mathrm{t}$. If we set $\beta_{U V}=\mathrm{K}_{0}$ and $\left(\beta_{V V}-\beta_{U V}\right)=\mathrm{K}_{1}$ then we can write:

$$
E\left(C_{V}\right)=S *\left(1-e^{-e^{\left(K_{0}+K_{1} * F_{V A}\right)} * \operatorname{Pr} e v A * \Delta t}\right)
$$

Since we will know the observed infection status of the specific badger $\left(\mathrm{C}_{\mathrm{V}}\right)$ at the end of the time interval $(\Delta t)$, we can fit a GLM with $\log (\operatorname{PrevA} \Delta \mathrm{t}$ ) as an offset and calculate $\mathrm{K}_{0}$ and $\mathrm{K}_{1}$. We can subsequently calculate $\beta_{U V}$ and $\beta_{V V}$ as:

$\beta_{U V}=\operatorname{Exp}\left[\mathrm{K}_{0}\right]$

$\beta_{V V}=\operatorname{Exp}\left[\mathrm{K}_{0}+\mathrm{K}_{1}\right]$

If we apply the same logic to model the expected infectious status of an unvaccinated badger $\left(\mathrm{C}_{\mathrm{U}}\right)$ that was trapped in zone $\mathrm{A}$ at time $\mathrm{t}$ and at time $\mathrm{t}+1$, we could estimate $\beta_{U U}$ and $\beta_{V U}$ as:

$$
E\left(C_{U}\right)=S *\left(1-e^{-e^{\left(k_{0}+k_{1} * F_{V A}\right)} * \operatorname{Pr} e v A * \Delta t}\right)
$$

where $\beta_{U U}=\operatorname{Exp}\left[\mathrm{k}_{0}\right]$ and $\beta_{V U}=\operatorname{Exp}\left[\mathrm{k}_{0}+\mathrm{k}_{1}\right]$

This way we can estimate the four betas based on incidence data from all three zones. In zone $\mathrm{C}$, there will be no (or very few) vaccinated badgers, so $\mathrm{F}_{\mathrm{VC}}$ will be zero and that zone does not contribute to the estimation of $\mathrm{k}_{1}$ and thus that area does not give information for $\beta_{\mathrm{Vu}}$.

Vaccine efficacy for susceptibility can be calculated then as: 


$$
V E_{S}=1-\left(\beta_{U V} / \beta_{U U}\right)
$$

We can also calculate vaccine efficacy for infectiousness as:

$$
V E_{I}=1-\left(\beta_{V U} / \beta_{U U}\right)
$$

Finally we can estimate $R(p)$ where $p$ is the proportion of vaccinated as:

$$
\left.R(p)=\frac{1}{2} *\left[(1-p) * \beta_{U U}+p^{*} \beta_{V V}+\sqrt{\left((1-p) * \beta_{U U}+p^{*} \beta_{V V}\right)^{2}+4 * p^{*}(1-p) * \beta_{U V} * \beta_{V U}}\right)\right] * T
$$

where $\mathrm{T}$ is the duration of the infectious period of a typical infected individual (for relative comparisons of $R(p)$ knowledge of T is not essential).

For decision making, if $\mathrm{p}=0$ and $\beta_{U U} \mathrm{~T}<1$, then no vaccination is needed; if $\mathrm{p}=1$ and $\beta_{V V} \mathrm{~T}>1$, vaccination will not stop spread of the disease. Otherwise, the vaccination fraction necessary in order to achieve $R(p)<1$ can be calculated.

\section{Concluding remarks}

The aim of this paper was to present the theory applied to the design of a badger vaccine trial, as well as the epidemiological methodology and statistical analysis that will help to interpret the results obtained.

The vaccine trial area was divided into three zones $\mathrm{A}, \mathrm{B}$ and $\mathrm{C}$, such that differences in infection pressure in the three zones at the beginning of the trial will be minimized. However, there is an inherent assumption that the contact patterns between badgers will be similar in the three zones. We believe that this is a reasonable assumption as the size of the total area is sufficiently small, and the landscape and distribution of setts is very similar in the three zones. Further, changes with respect to infection pressure in the three zones will occur as the vaccine starts working. Our model is designed to adjust for these changes. For simplicity, we have only presented the analysis relevant to badgers repeated trapped in the same zone. However, the final model can be modified to accommodate other scenarios where badgers move from one zone to another.

Where possible, natural boundaries are used to define the perimeter of the study area. The internal boundaries between the three zones include streams, rivers and roads. The external boundaries, while not bio-secure, should be substantial enough to 
delineate badger territories. This would reduce the extent of typical badger movement into and out of the study area.

Defining specific questions of interest in the early stages of the vaccine trial was considered crucial. Estimation of the reproduction ratio $(R(p))$ calculated as a function of the vaccination coverage $(p)$ will give us invaluable information on the impact of vaccination in disease transmission and dynamics of $M$. bovis infection in badgers. The importance of considering the potential indirect effects associated with the vaccination program in badgers has been highlighted in the paper; furthermore, it has been shown how to estimate $R(p), \mathrm{VE}_{\mathrm{S}}$ and $\mathrm{VE}_{\mathrm{I}}$ by using different vaccination coverages in the study area.

Although the vaccine trial will not determine whether bTB can be eradicated in cattle and badgers through a strategy of badger vaccination, the parameters obtained in the vaccine trial will be used in a mathematical model of bTB transmission (currently under development) in order to assess different control and eradication options for bTB in cattle in the Republic of Ireland.

\section{References}

Aldwell, F. E., Keen, D.L., Parlane, N.A., Skinner, M.A., de Lisle, G.W., Buddle, B.M., 2003a. Oral vaccination with Mycobacterium bovis BCG in a lipid formulation induces resistance to pulmonary tuberculosis in brushtail possums. Vaccine 22, 70-76.

Aldwell, F.E., Tucker, I.G., de Lisle, G.W., Buddle, B.M., 2003b. Oral delivery of Mycobacterium bovis BCG in a lipid formulation induces resistance to pulmonary tuberculosis in mice. Infection and Immunity 71, 101-108.

Barrow, P.A., Gallagher, J., 1981. Aspects of the epidemiology of bovine tuberculosis in badgers and cattle. I. The prevalence of infection in two wild animal populations in south-west England. J. Hyg. (Lond) 86, 237-245.

Buddle, B.M., Aldwell, F.E., Kee, D.L., Parlane, N.A., Hamel, K.L., de Lisle, G.W., 2006. Oral vaccination of brushtail possums with BCG: investigation into factors that may influence vaccine efficacy and determination of duration of protection. N.Z. Vet. J. 54, 224-230.

Cheeseman, C.L., Jones, G.W., Gallagher, J., Mallinson, P.J., 1981. The population structure, density and prevalence of tuberculosis (Mycobacterium bovis) in badgers (Meles meles) from four areas in south-west England. J. Appl. Ecol. 18, 795-804.

Corner, L.A.L., Buddle, B.M., Pfeiffer, D.U., Morris, R.S., 2001. Aerosol vaccination of the brushtail possum (Trichosurus vulpecula) with bacille Calmette-Guérin: the duration of protection. Vet. Microbiol. 81, 181-191. 
Corner, L.A.L., Norton, S., Buddle, B.M., Morris, R.S., 2002. The efficacy of bacille Calmette-Guérin vaccine in wild brushtail possums (Trichosurus vulpecula). Res. Vet. Sci. 73, 145-152.

Corner, L.A.L., Costello, E., Lesellier, S., O'Meara, D., Sleeman, D.P., Gormley, E., 2007. Experimental tuberculosis in the European badger after endobronchial inoculation of Mycobacterium bovis: I. Pathology and bacteriology. Res. Vet. Sci. 83, 53-62.

Corner, L.A., Costello, E., Lesellier, S., O'Meara, D., Gormley, E., 2008. Vaccination of European badgers (Meles meles) with BCG by the subcutaneous and mucosal routes induces protective immunity against endobronchial challenge with Mycobacterium bovis. Tuberculosis (Edinb) 88, 601-609.

De Jong, M.C.M., van der Poel, W.H.M., Kramps, J.A., Brand, A. and van Oirschot, J.T., 1996. Quantitative investigation of population persistence and recurrent outbreaks of bovine respiratory syncytial virus on dairy farms. Am. J. Vet. Res. 57, 628-633.

Diekmann, O., Heesterbeek, J.A.P., 2000. Mathematical epidemiology of infectious diseases: model building, analysis and interpretation. Wiley series in mathematical and computational biology. Chichester, UK: Wiley.

Eves, J.A., 1999. Impact of badger removal on bovine tuberculosis in east County Offaly. Irish Vet. J. 52, 199-203.

Fagan, J., 1993. Tuberculosis in badgers in Ireland: Pathology. In: The badger. Proceedings of a seminar held on 6-7 March 1991 pp. 117-122.

Gallagher, J., Monies, R., Gavier-Widen, M., Rule, B., 1998. Role of infected, nondiseased badgers in the pathogenesis of tuberculosis in the badger. Vet. Rec. 142, 710-714.

Griffin, J.M., Dolan, L.A., 1995. The role of cattle-to-cattle transmission of Mycobacterium bovis in the epidemiology of tuberculosis in cattle in the Republic of Ireland: a review. Irish Vet. J. 48, 228-234.

Griffin, J.M., Williams, D.H., Kelly, G.E., Clegg, T.A., O'Boyle, I., Collins, J.D., More, S.J., 2005. The impact of badger removal on the control of tuberculosis in cattle herds in Ireland. Prev. Vet. Med. 67, 237-266.

Halloran, M.E., Struchiner, C.J., Longini, I.M., Jr., 1997. Study Designs for Evaluating Different Efficacy and Effectiveness Aspects of Vaccines. Am. J. Epidemiol. 146, 789-803.

Halloran, M.E., Longini, I.M., Jr., Struchiner, C.J., 1999. Design and Interpretation of Vaccine Field Studies. Epidemiol. Rev. 21, 73-88.

Jenkins, H.E., Woodroffe, R., Donnelly, C.A., 2008. The effects of annual widespread badger culls on cattle tuberculosis following the cessation of culling. Int. J. Infect. Dis. 12, 457465.

Krebs, J.R., 1997. Bovine Tuberculosis in Cattle and Badgers. Ministry of Agriculture, Fisheries and Food, London. UK, 191. 
Lesellier, S., Corner, L., Costello, E., Lyashchenko, K., Greenwald, R., Esfandiari, J., Singh, M., Hewinson, R.G., Chambers, M., Gormley, E., 2009. Immunological responses and protective immunity in BCG vaccinated badgers following endobronchial infection with Mycobacterium bovis. Vaccine 27, 402-409.

Longini, I.M., Jr., Sagatelian, K., Rida, W.N., Halloran, M.E., 1998. Optimal vaccine trial design when estimating vaccine efficacy for susceptibility and infectiousness from multiple populations. Stat. Med. 17, 1121-1136.

More, S.J., Good, M., 2006. The tuberculosis eradication programme in Ireland: a review of scientific and policy advances since 1988. Vet. Microbiol. 112, 239251.

Murphy, D., Gormley, E., Costello, E., O'Meara, D., Corner, L.A.L., 2010. The prevalence and distribution of Mycobacterium bovis infection in European badgers (Meles meles) as determined by enhanced post mortem examination and bacteriological culture. Res. Vet. Sci 88, 1-5.

Noonan, N.L., Sheane, W.D., Harper, L.R., Ryan, P.J., 1975. Wildlife as a possible reservoir of bovine TB. Irish Vet. J. 29, 1.

Olea-Popelka, F.J., Fitzgerald, P., White, P., McGrath, G., Collins, J.D., O'Keeffe, J., Kelton, D.F., Berke, O., More, S., Martin, S.W., 2009, Targeted badger removal and the subsequent risk of bovine tuberculosis in cattle herds in county Laois, Ireland. Prev. Med. Vet. 88, 178-184.

Pope, L.C., Butlin, R.K., Wilson, G.J., Woodroffe, R., Erven, K., Conyers, C.M., Chris, M., Franklin, T., Delahay, R.J., Cheeseman, C.L., Chris, L., Burke, T., 2007. Genetic evidence that culling increases badger movement: implications for the spread of bovine tuberculosis. Mol. Ecol. 16, 4919-4929.

Tompkins, D.M., Ramsey, D.S.L., Cross, M.L., Aldwell, F.E., de Lisle, G.W., Buddle, B.M., 2009. Oral vaccination reduces the incidence of tuberculosis in free-living brushtail possums. Proc. R. Soc. B 276, 2987-2995.

Woodroffe, R., Gilks, P., Johnston, W.T., Le Fevre, A.M., Cox, D.R., Donnelly, C.A., Bourne, F.J., Cheeseman, C.L., Gettinby, G., MCinerney, J.P., Morrison, W.I., 2007. Effects of culling on badger abundance: implications for tuberculosis control. J. Zool. 28-37.

Watchorn, R.C., 1965. Bovine tuberculosis eradication scheme 1954-1965. Department of Agriculture and Fisheries. Dublin. 

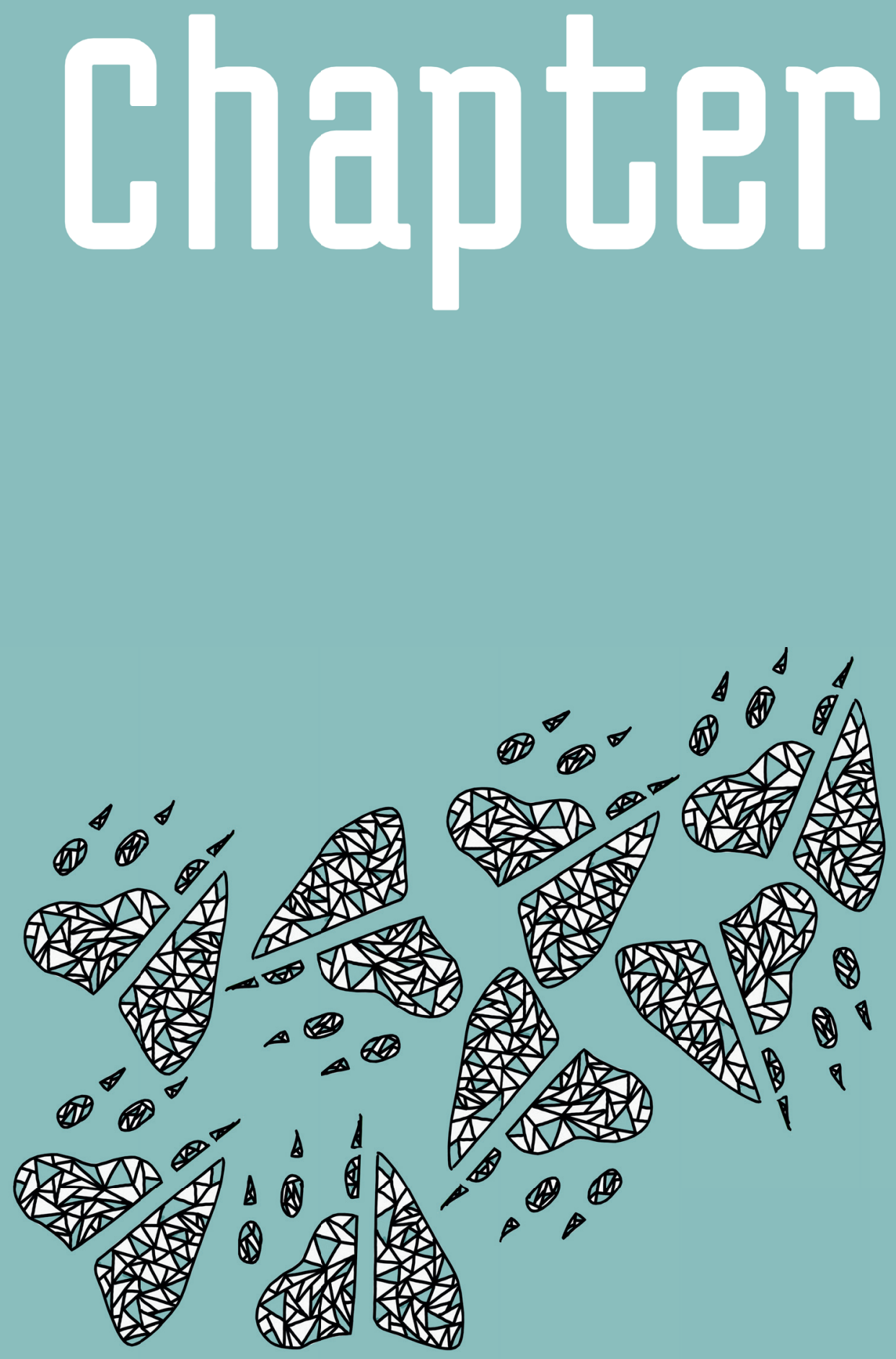

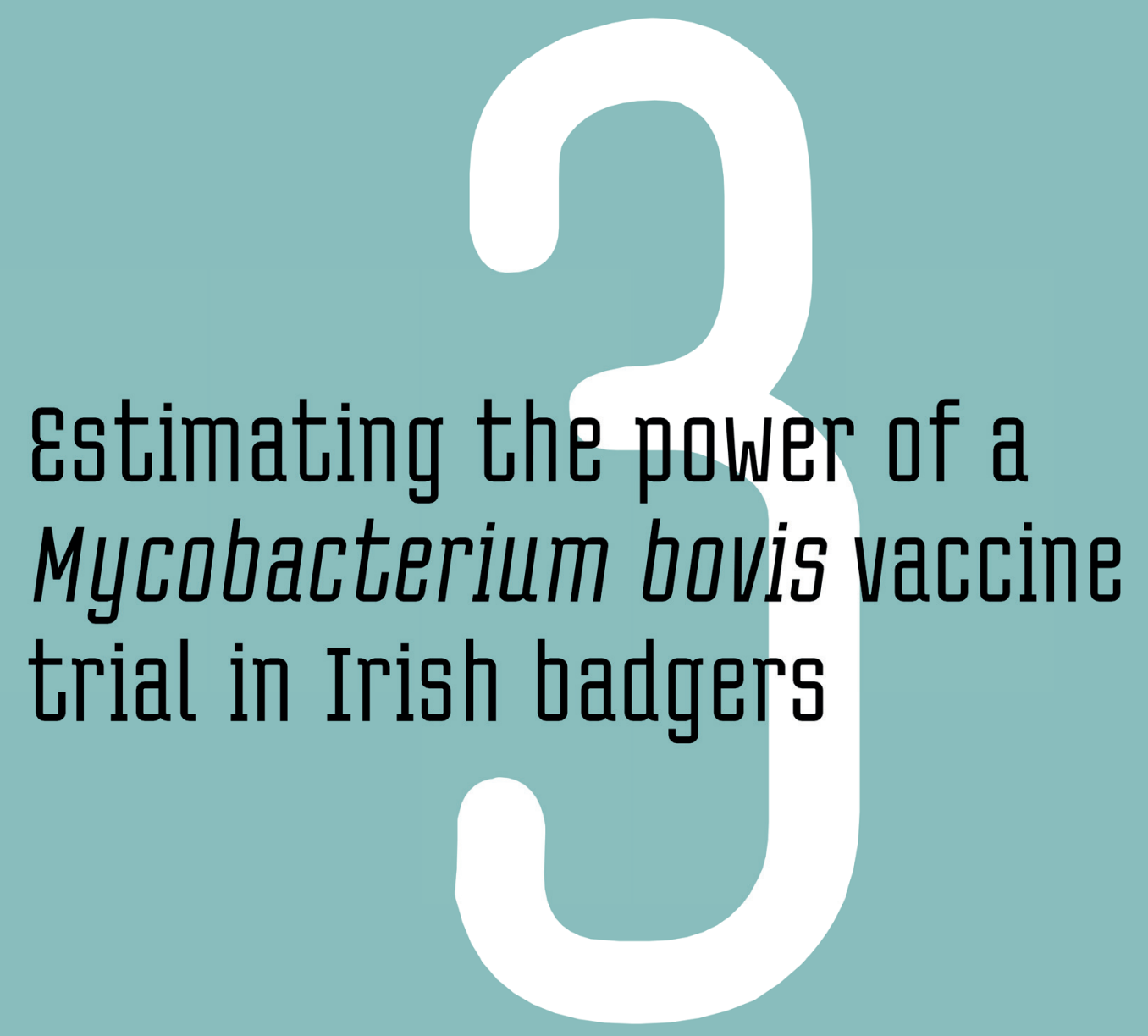

Aznar, I. , More, S.J. , Frankena, K. , de Jong, M.C.M. 


\section{Chapter 3}

\section{Estimating the power of a Mycobacterium bovis vaccine trial in Irish badgers}

Aznar, I., More, S.J., Frankena, K., de Jong, M.C.M.

Preventive Veterinary Medicine (2013) 111, 297-303

(C) 2013 Elsevier B.V. All rights reserved. 


\begin{abstract}
The aim of this study was to estimate the power, using simulation techniques, of a group randomized vaccine field trial designed to assess the effect of vaccination on Mycobacterium bovis transmission in badgers. The effects of sample size (recapture percentage), initial prevalence, sensitivity and specificity of the diagnostic test, transmission rate between unvaccinated badgers, Vaccine Efficacy for Susceptibility $\left(\mathrm{VE}_{\mathrm{S}}\right)$ and Vaccine Efficacy for Infectiousness $\left(\mathrm{VE}_{\mathrm{I}}\right)$, on study power were determined.

Sample size had a small effect on power. Study power increased with increasing transmission rate between non-vaccinated badgers. Changes in $\mathrm{VE}_{S}$ had a higher impact on power than changes in $\mathrm{VE}_{\mathrm{I}}$. However, the largest effect on study power was associated with changes in the specificity of the diagnostic test, within the range of input values that were used for all other modelled parameters. Specificity values below $99.4 \%$ yielded a study power below $50 \%$ even when sensitivity was $100 \%$ and, $\mathrm{VE}_{\mathrm{I}}$ and $\mathrm{VE}_{\mathrm{S}}$ were both equal to $80 \%$. The effect of changes in sensitivity on study power was much lower.

The results from our study are in line with previous studies, as study power was dependent not only on sample size but on many other variables. In this study, additional variables were studied i.e. test sensitivity and specificity. In the current vaccine trial, power was highly dependent on the specificity of the diagnostic test. Therefore, it is critical that the diagnostic test used in the badger vaccine trial is optimized to maximise test specificity.
\end{abstract}




\section{Introduction}

Badgers (Meles meles) are an important reservoir of Mycobacterium bovis for cattle in Ireland and the United Kingdom and as a result, eradication of bovine tuberculosis (bTB) will be highly unlikely without measures to prevent transmission between cattle and badgers and vice versa (More, 2009). In a recent Irish study, 36\% of badgers were found to be infected with bTB (Murphy et al., 2010), with prevalence known to vary in areas of high (43.2\%, Corner et al., 2012) and low (14.9\%, Murphy et al., 2011) bTB prevalence in cattle. Focused badger culling is currently being used as a short-to-medium term strategy to limit transmission in areas of high bTB prevalence in cattle, with the expectation that culling will be replaced by badger vaccination once an effective bTB vaccine becomes available (Sheridan, 2011). In 2001, Ireland initiated a 10-year work programme investigating the use of Bacillus Calmette-Guerin (BCG) vaccine in badgers as a medium-long term strategy to assist with national bTB control and eradication (Corner et al., 2007; Lesellier et al., 2009). Based on a series of initial studies in captive badgers, BCG vaccination in badgers was associated with a reduction in both the number and size of gross histological lesions (Corner et al., 2007, 2008a,b, 2010). These pen-based studies were recently extended to the field, with the design and implementation of a field trial in Ireland to evaluate vaccine efficacy in wild badger populations (Aznar et al., 2011).

In traditional vaccine field trials, individuals are randomly allocated (individual randomization) to either a vaccine or a placebo treatment and the relative risk of acquiring infection is determined by comparing infection rates in vaccinated and non-vaccinated individuals. This design is appropriate for non-communicable diseases because the probability that an individual will become infected depends only on their susceptibility. Individual randomized trials allow the estimation of vaccine effects that reduce the susceptibility of an individual to infection or Vaccine Efficacy for Susceptibility ( $\mathrm{VE}_{\mathrm{S}}$ ), also known as the direct effect of vaccination (Halloran et al., 1999). When dealing with infectious diseases, however, the likelihood that an individual will become infected depends not only on its susceptibility but also on the infectivity of surrounding individuals. The reduction in infectivity achieved by vaccination is known as Vaccine Efficacy for Infectiousness $\left(\mathrm{VE}_{\mathrm{I}}\right)$, and is the result of the indirect effects of the vaccination on vaccinated and non-vaccinated individuals, of which herd immunity is the most important. With infectious diseases, group randomized trials are the design of choice, allowing estimates of both the reduction in susceptibility (VES) and infectiveness ( $\left.\mathrm{VE}_{\mathrm{I}}\right)$ (Riggs and Koopman, 2005). In a field trial to evaluate BCG vaccine efficacy in badgers, Aznar et al. (2011) outlined the use of group randomization to provide estimates of both $\mathrm{VE}_{\mathrm{S}}$ and $\mathrm{VE}_{\mathrm{I}}$ based on incidence data from three badger populations vaccinated with BCG at different levels of vaccination coverage: $100 \%, 50 \%$ and $0 \%$. In such a 
trial, estimates of $\mathrm{VE}_{\mathrm{I}}$ may be particularly important, given the reported reduction in gross histological lesions (and, potentially, reduced infectiousness) in vaccinated badgers (Hayes et al., 2000; Corner et al., 2008a). This trial design is similar to that outlined by Longini et al. (1998), who propose methodology, using unconditional parameters, to jointly estimate $\mathrm{VE}_{\mathrm{S}}$ and $\mathrm{VE}_{\mathrm{I}}$ from a trial conducted in only two populations. This approach, as used by Longini et al. (1998) and Aznar et al. (2011), has been defined by Hayes et al. (2000) as a hybrid of group and individually randomised trials.

As outlined by Charvat et al. (2009), power calculations based on the comparison of two independent binomials can largely overestimate study power if indirect effects are not taken into account. In recognition of this concern, there have been recent changes in both the design and analysis of vaccine trials to for estimate sample size and power in these studies. In particular, computer simulation techniques are now frequently used to address study power issues (Walters, 2004; Barth-Jones et al., 2004).

In group randomized trials, where direct and indirect vaccine effects are each important, power depends on a range of factors. Riggs and Koopman $(2004,2005)$ examined some of these factors, including unit (group) size, contact rate, external force of infection and infection duration.

In this paper, we estimate the power, using simulation techniques, of a group randomized vaccine field trial designed to assess the effect of vaccination on $M$. bovis transmission in badgers. In this work, study power was defined as the proportion of simulations in which the null hypothesis (that the transmission parameter between vaccinated $\left(\beta_{V V}\right)$ and non-vaccinated badgers $\left(\beta_{U U}\right)$ was equal) was rejected at a 0.05 level of significance. Further, we assess the effects on study power of sample size (recapture percentage), initial prevalence, the sensitivity and specificity of the diagnostic test, transmission rate between badgers prior to the start of the trial, and $\mathrm{VE}_{\mathrm{S}}$ and $\mathrm{VE}_{\mathrm{I}}$.

\section{Material and Methods}

\subsection{Vaccine trial design}

The vaccine trial area, covering approximately $750 \mathrm{~km}^{2}$ in county Kilkenny, was divided into three zones (A, B and C) north to south, each with similar characteristics in terms of size, number of main badger setts, cattle herds, cattle and land classification type. Three vaccination levels, $100 \%, 50 \%$ and $0 \%$, were allocated to zones $\mathrm{A}, \mathrm{B}$ and $\mathrm{C}$ to achieve a north-south gradient in vaccination coverage. The middle zone (zone B) was vaccinated using a capture-tag-release protocol, to achieve 
and maintain $50 \%$ vaccination coverage throughout the trial period. In this zone, randomisation was conducted at the level of the animal (Aznar et al., 2011). Zones $\mathrm{A}$ and $\mathrm{C}$ were randomly allocated to a $100 \%$ and $0 \%$ vaccination coverage.

The vaccination trial commenced in September 2009 and ran for four years. During each of the first 3 years, there were two trapping exercises over the entire trial area. At their first trapping, each badger was allocated to a vaccine/placebo treatment. A blood sample was taken at each capture during the trial period; however, treatment was repeated no more than once each year. During year 4, two trappings exercises were also conducted. At their first capture in year 4, a blood sample was collected, and each badger was either vaccinated or euthanized for detailed post mortem investigation. Data from the second trapping carried out in year 4 was not included in the analysis. Therefore, each badger could be captured, at most, on 7 separate occasions.

\subsection{The model}

The outcome from the vaccine trial will be of the form of a Bernoulli experiment. Data were collected about each badger at each trapping, including the infection status of each animal at the initial and each subsequent trapping.

Further to Aznar et al. (2011), the expected number of vaccinated $E\left(C_{V}\right)$ and nonvaccinated $\left(\mathrm{E}\left(\mathrm{C}_{\mathrm{U}}\right)\right.$ badgers in each of the study zones that became infected with $M$. bovis between consecutive trappings can be estimated as:

$$
\begin{aligned}
& E\left(C_{V}\right)=S_{V} *\left(1-e^{-\left(K_{0}+K_{1} * F v z\right) * \operatorname{Prev} Z * \Delta t}\right) \text { and } \\
& E\left(C_{U}\right)=S_{U} *\left(1-e^{-\left(k_{0}+k_{1} * F v z\right) \operatorname{Pr} e v Z * \Delta t}\right) \text {, respectively. }
\end{aligned}
$$

where $\mathrm{K}_{0}=\beta_{U V}, \mathrm{~K}_{1}=\left(\beta_{V V}-\beta_{U V}\right), \mathrm{k}_{0}=\beta_{U U}$ and $\mathrm{k}_{1}=\left(\beta_{V U}-\beta_{U U}\right)$.

$\mathrm{S}_{\mathrm{V}}$ and $\mathrm{S}_{\mathrm{U}}$ are the number of susceptible vaccinated and non-vaccinated badgers observed at the previous trapping exercise, $\mathrm{F}_{\mathrm{VZ}}$ is the fraction of infected badgers that are vaccinated and PrevZ is the average prevalence of infection in the zone selected (A, B or C). $\Delta \mathrm{t}$ is the time between two consecutive trappings.

As the number of new infections will be inferred from available serology, by fitting a GLM with $\log \left(\operatorname{Prev} Z^{*} \Delta t\right.$ ) as an offset, Aznar et al. (2011) showed how to calculate $\mathrm{K}_{0}, \mathrm{~K}_{1}, \mathrm{k}_{0}$ and $\mathrm{k}_{1}$. The four transmission parameters can then be calculated as: $\beta_{U V}=\operatorname{Exp}\left[\mathrm{K}_{0}\right], \beta_{V V}=\operatorname{Exp}\left[\mathrm{K}_{0}+\mathrm{K}_{1}\right], \beta_{U V}=\operatorname{Exp}\left[\mathrm{k}_{0}\right]$ and $\beta_{V U}=\operatorname{Exp}\left[\mathrm{k}_{0}+\mathrm{k}_{1}\right]$.

To estimate study power, only $\beta_{V V}$ and $\beta_{U U}$ are needed.

\subsection{The simulation process}




\subsubsection{Input data}

In order to estimate $\beta_{V V}$ and $\beta_{U U}$, each of the following were added as input data in the simulation process:

- the prevalence (PrevZ) prior to the commencement of the study and at every time interval $(\Delta t)$,

- the fraction of infected badgers that were vaccinated at the end of every time interval ( $F_{V Z}$ denoted as fi in SAS program, see supplementary file) in each of the study zone, and the fraction of susceptible badgers that were vaccinated at the end of every time interval ( $\mathrm{F}_{\mathrm{SZ}}$ denoted as fs in SAS program) in each study zone. Both fractions $\mathrm{F}_{\mathrm{VZ}}$ and $\mathrm{F}_{\mathrm{SZ}}$ had the same values,

- the total number of badgers trapped in each study zone at each trapping exercise (Total_n),

- the recapture percentage; of the badgers caught during the current trapping exercise, this was the percentage that had been caught at least once during a previous trapping exercise (Trapperc),

- the transmission parameter among non-vaccinated badgers at the beginning of the study $\left(\beta_{U U}\right)$,

- the Vaccine Efficacy for Susceptibility $\left(\mathrm{VE}_{\mathrm{S}}\right)$ and Vaccine Efficacy for Infectiousness $\left(\mathrm{VE}_{\mathrm{I}}\right)$, and

- the sensitivity (Se) and specificity ( $\mathrm{Sp}$ ) of the serological test.

\subsubsection{Simulation flow}

A dataset of 36 entries ( 6 subsequent trappings $\mathrm{x} 3$ study zones $\mathrm{x} 2$ vaccination status) was created (see the supplementary file for information about the data). Each entry included trapping number (2-7), study zone (A to $\mathrm{C}$, coded as 1-3), the vaccination status $(0=$ non vaccinated: $1=$ vaccinated $)$, fi, fs, PrevZ and Total_n.

We assumed that none of the badgers trapped were vaccinated in zone A and that all badgers trapped were vaccinated in zone $\mathrm{C}$. For each subsequent trapping (i.e. all but the first trapping) and for each study zone, the number of new infections within vaccinated and non-vaccinated badgers (variables $\mathrm{E}\left(\mathrm{C}_{\mathrm{V}}\right)$ and $\mathrm{E}\left(\mathrm{C}_{\mathrm{U}}\right)$ respectively) were simulated using the cumulative binomial distribution with parameters $(s, p)$, where $s$ is the total number of susceptible badgers caught in each of the trial zones at the beginning of each time interval $\Delta t$, and $p$ is the probability that each of these badgers will become infected during that time interval.

The parameter $s$ was calculated separately for vaccinated $\left(\mathrm{S}_{\mathrm{V}}\right)$ and non-vaccinated badgers $\left(\mathrm{S}_{\mathrm{U}}\right)$ as:

$\left(\mathrm{S}_{\mathrm{v}}\right)=$ Total_n $*$ Trapperc* $\mathrm{F}_{\mathrm{VZ}} *(1-\mathrm{PrevZ})$

and 
$\left(\mathrm{S}_{\mathrm{U}}\right)=$ Total_ $\mathrm{n} *$ Trapperc* $\left(1-\mathrm{F}_{\mathrm{VZ}}\right) *(1-\mathrm{PrevZ})$

and the probability of becoming infected was calculated as:

$p=1-e^{-e^{\left(K_{0}+K_{1} * F_{V Z}\right)} * \operatorname{Pr} e v Z * \Delta t}$

The initial transmission parameter $\beta_{U U}$, needed to estimate $p$ was set within the SAS program (see the supplementary file). The values of $\beta_{U V}$ (transmission parameter from a non-vaccinated to a vaccinated badger) and $\beta_{\mathrm{vU}}$ (transmission parameter from a vaccinated to a non-vaccinated badger) were determined based on the assumed reduction in $\beta_{U U}$ given by the values of $\mathrm{VE}_{\mathrm{S}}$ and $\mathrm{VE}_{\mathrm{I}}$. So, for instance, if we assume VEs $=80 \%$, and a $\beta_{U U}=0.1$, then there will be a reduction of $80 \%$ in the transmission parameter $\beta_{\mathrm{Uv}}$ with respect to $\beta_{U U}$, so $\beta_{U V}=0.02$. The initial transmission parameter within vaccinated badgers $\left(\beta_{V V}\right)$ was calculated as:

$\beta_{V V}=\beta_{U V} * \beta_{V U}$

The number of new infections $\mathrm{E}\left(\mathrm{C}_{\mathrm{V}}\right)$ and $\mathrm{E}\left(\mathrm{C}_{\mathrm{U}}\right)$ was then refined depending on the $\mathrm{Sp}$ and $\mathrm{Se}$ of the diagnostic test, taking into account the number of false positives and false negatives.

By fitting a GLM (binomial distribution, complementary loglog link function) with $\log \left(\operatorname{PrevZ} Z^{*} \Delta \mathrm{t}\right)$ as an offset, $\beta_{U U}$ and $\beta_{V V}$ were estimated and study power determined by means of a paired t-test.

\subsubsection{Output}

The dataset was read into SAS (SAS 9.1, SAS Institute Inc., Cary, NC, USA) and a simulation process was set up by means of a macro. We performed 1,000 simulations for each set of conditions (see section 2.3.4). Study power was defined as the proportion of simulations in which the null hypothesis $\left(\beta_{V V}=\beta_{U U}\right)$ was rejected at the 0.05 level of significance.

A three dimensional graph showing the power of the study for all combinations of sensitivity (Se) between 10 and $100 \%$, and specificity (Sp) between 98 and $100 \%$ at increments of $5 \%$ and $0.2 \%$, respectively, was built using Mathematica ${ }^{\circledR} 6.0$ (Wolfram Research, Champaign, IL, USA). We assumed an initial transmission between non-vaccinated badgers of $\beta_{U U}=0.1$ and a vaccine effect on susceptibility $\left(\mathrm{VE}_{\mathrm{S}}\right)$ and infectivity $\left(\mathrm{VE}_{\mathrm{I}}\right)$ of $80 \%$. To create the graph, we fitted a generalized linear model (GLM) with different links of the binomial family (Stata ${ }^{\circledR}$ version 10; Stata Corp, College Station, TX, USA) to obtain the function that fitted our data best. The link with the lowest AIC was selected.

\subsubsection{Sensitivity analysis}




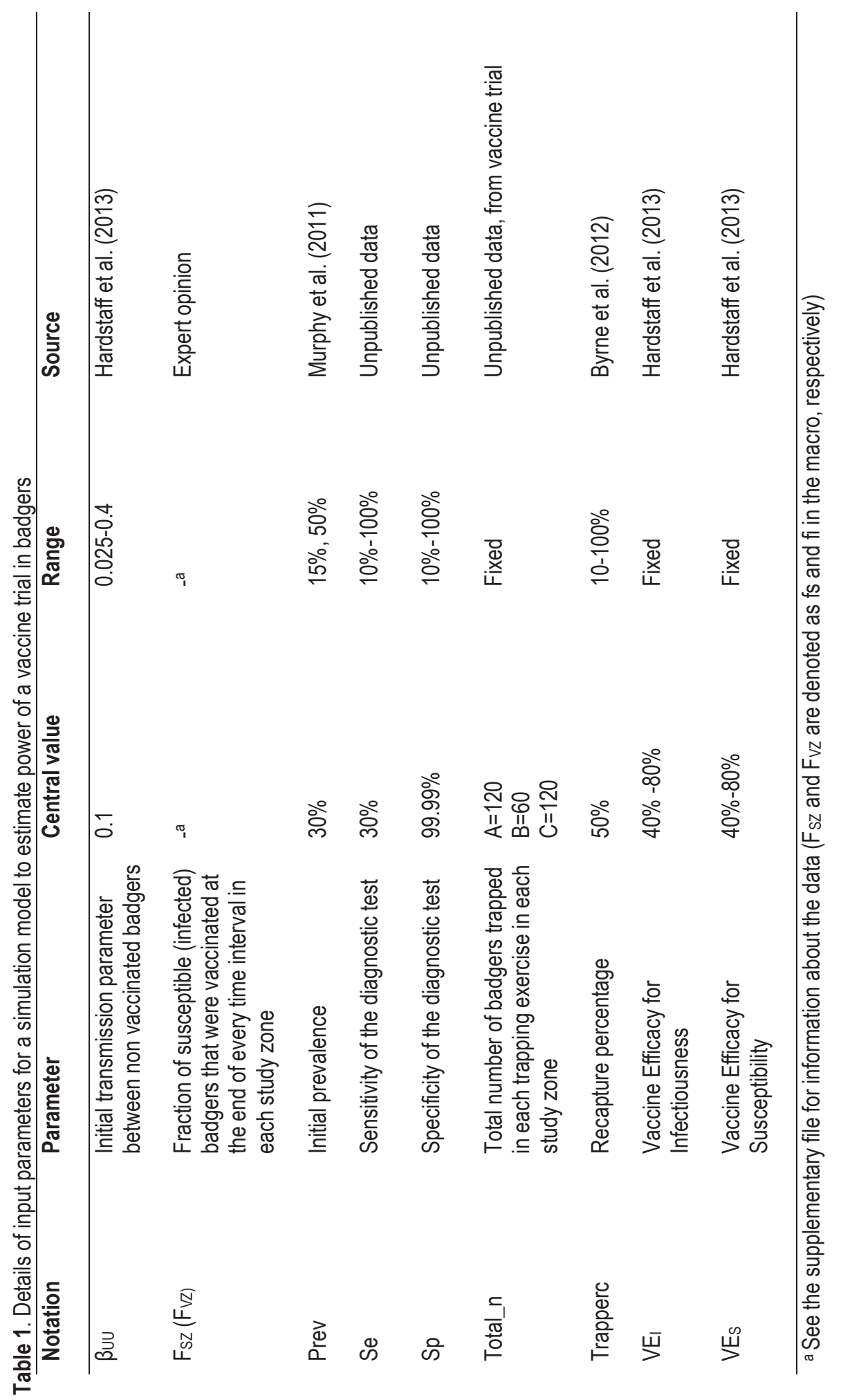


In the sensitivity analysis, the central value and range for each parameter was chosen as follows:

- With Se and Sp of the diagnostic test, all possible values (that is, from $10 \%$ to $100 \%$ ) were considered, at increments of $5 \%$.

- For all other parameters, the selection of values was determined following a review of relevant literature or, if not available, expert opinion. If knowledge was lacking, a conservative approach was taken, when considering the central value and the likely ranges.

To elaborate (see Table 1):

- Sample size is defined in our study as the total number of badgers that were recaptured (Total $\mathrm{n} *$ Trapperc). In the simulation exercise, the total number of badgers trapped (Total_n) was 300, including 120, 60 and 120 in zones A, $\mathrm{B}$ and $\mathrm{C}$, respectively. These numbers were based on the figures obtained during the first trapping exercise $(120,64$ and 115 badgers in zones A, B and $\mathrm{C}$, respectively; unpublished data). The percentage of badgers re-trapped was set at $50 \%$ based on expert opinion, and recapture values ranging from 10 to $100 \%$ were also evaluated.

- The initial prevalence for the three trial zones was set at $30 \%$ based on an average from Murphy et al. (2011), and values of 15 and 50\% were also evaluated.

- The initial transmission parameter $\beta_{U U}$ was set to 0.1 , being an approximate average of the inter- and intra-group transmission parameters described by Hardstaff et al. (2012). In the sensitivity analysis, values of 0.4, 0.2, 0.1, 0.05 and 0.025 were also evaluated.

\section{Results}

\subsection{Effect of sensitivity and specificity of the diagnostic test on study power}

The study power for varying Se and Sp values of the diagnostic test is shown in Fig. 1. A decrease in Sp had a much larger impact on power than a decrease in Se. The power of the study decreased sharply to $15 \%$ or lower when the $\mathrm{Sp}$ is equal or lower than $98.0 \%$, independent of Se. The effect of Se on study power was much less; given a $\mathrm{Sp}$ of 98.8 , study power remained above $50 \%$ even when Se was $40 \%$. In these calculations, $\mathrm{VE}_{\mathrm{S}}$ and $\mathrm{VE}_{\mathrm{I}}$ were each assumed to equal $80 \%$, however, similar results were obtained when $\mathrm{VE}_{\mathrm{S}}$ and $\mathrm{VE}_{\mathrm{I}}$ were assumed to be $40 \%$. 


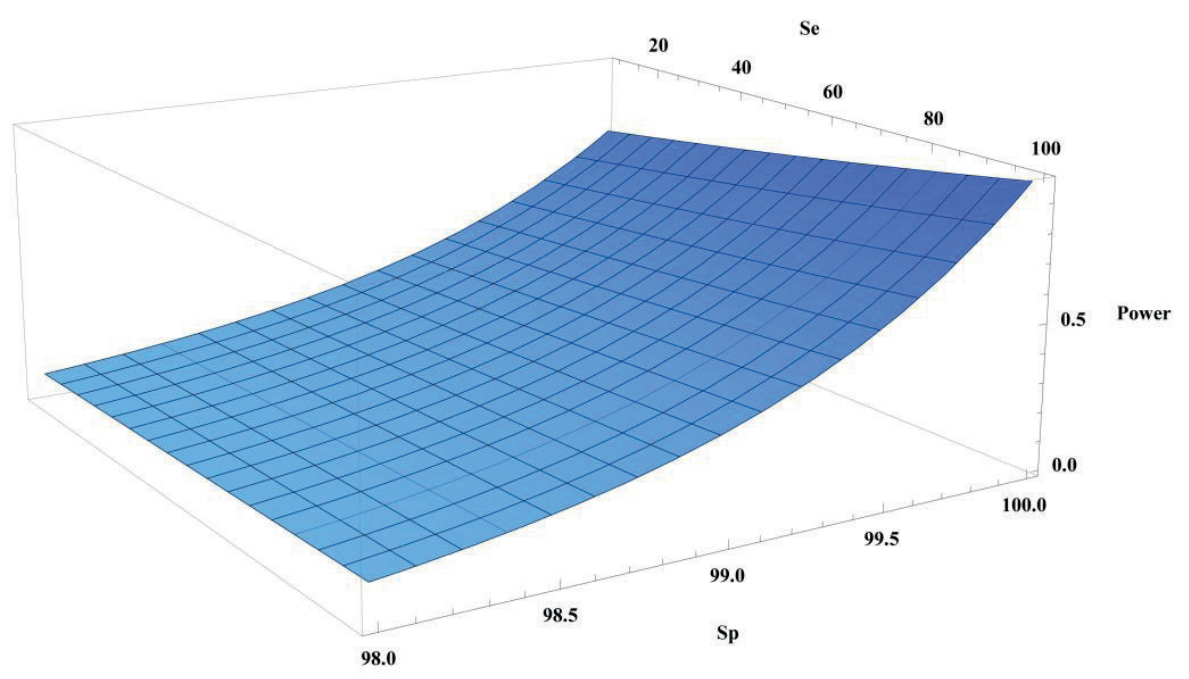

Fig. 1. Three dimensional plot showing the power of the badger vaccine trial as a function of the sensitivity and specificity of the diagnostic test. $\beta_{U U}$ was set at 0.1 , re-trapping percentage was set at $50 \%$, and $\mathrm{VE}_{\mathrm{I}}$ and $\mathrm{VE}_{\mathrm{S}}$ at $80 \%$.

\subsection{Effect of variations in sample size on study power}

Assuming a Se of $30 \%$, a Sp of $99.99 \%$ and a transmission parameter for nonvaccinated badgers $\beta_{U U}=0.1$, the study power was determined for two scenarios: VES and $\mathrm{VE}_{\mathrm{I}}$ both equal to $40 \%$ and $80 \%$, respectively, when the percentage of total badgers re-trapped varied from $10 \%$ to $100 \%$. Study power increased from $40 \%$ to $85 \%$ as recapture percentage increased from $10 \%$ to $40 \%$, but did not increase further as recapture percentage increased from 40 to $100 \%$. When $\mathrm{VE}_{\mathrm{S}}$ and $\mathrm{VE}_{\mathrm{I}}$ were each set to $40 \%$, study power increased from $40 \%$ to $77 \%$ as sample size increased from $10 \%$ to $60 \%$, with little further change as sample size increased above $60 \%$.

\subsection{Effect of variations in the initial transmission rate on study power}

The impact on study power of changes in transmission rate between badgers prior to the start of the vaccine trial $\left(\beta_{U U}\right)$ was assessed. In all previous calculations, a transmission value of 0.1 had been used. Assuming $\mathrm{Se}=30 \%, \mathrm{Sp}=99.99 \%$, and $\mathrm{VE}_{\mathrm{S}}$ and $\mathrm{VE}_{\mathrm{I}}=80 \%$, study power increased from $84 \%$ to $90 \%$ as $\beta_{U U}$ increased from 0.1 to 0.4 , and decreased to $48 \%$ when $\beta_{\mathrm{UU}}$ was 0.025 . Under equivalent conditions but assuming a $\mathrm{VE}_{\mathrm{S}}$ and $\mathrm{VE}_{\mathrm{I}}$ of $40 \%$, power varied from $49 \%$ to $76 \%$ as $\beta_{U U}$ increased from 0.025 to 0.1 and then remained stable. Study power was on average $77 \%$ and $68 \%$ when assuming an $80 \%$ and $40 \%$ reduction in $\mathrm{VE}_{\mathrm{S}}$ and $\mathrm{VE}_{\mathrm{I}}$, respectively. In 
both cases, the power decreased with a reduction in the transmission rate between non-vaccinated badgers.

\subsection{Effect of variations in $V E_{I}$ and $V E_{S}$ on study power}

To illustrate whether changes in $\mathrm{VE}_{\mathrm{S}}$ had a higher, lower or equal impact on power than changes in $\mathrm{VE}_{\mathrm{I}}$, the simulation process was run for the same range of $\beta_{U U}$ values as previously, but keeping either $\mathrm{VE}_{\mathrm{I}}$ or $\mathrm{VE}_{\mathrm{S}}$ at $80 \%$ while decreasing the opposite vaccine effect to $40 \%$. Using a $\mathrm{Se}=30 \%$ and $\mathrm{Sp}=99.99 \%$, the average study power when $\mathrm{VE}_{\mathrm{I}}$ and $\mathrm{VE}_{\mathrm{S}}$ were $80 \%$ and $40 \%$, respectively, was $69 \%$, compared to an average of $77 \%$ when $\mathrm{VE}_{\mathrm{I}}$ and $\mathrm{VE}_{\mathrm{S}}$ were $40 \%$ and $80 \%$, respectively. By comparing these two averages with the average obtained when both vaccine effects are set to $80 \%$ (average power $=77 \%$ ), we found that a decrease in $\mathrm{VE}_{\mathrm{S}}$ has a higher impact on study power than a decrease in $\mathrm{VE}_{\mathrm{I}}$.

\subsection{Effect of variations in initial prevalence on study power}

The study power was assessed assuming an initial prevalence of $15 \%$ and $45 \%$. When the initial prevalence was $45 \%$, and both $\mathrm{VE}_{\mathrm{S}}$ and $\mathrm{VE}_{\mathrm{I}}$ were set to $80 \%$, the study power obtained was $88 \%$. The power decreased to $77 \%$ when both vaccine efficacies were set to $40 \%$. When the initial prevalence assumed was $15 \%$ and vaccine efficacies were set to $80 \%$ the power obtained was $78 \%$ and this power decreased to $68 \%$ when vaccine efficacies were $40 \%$.

\section{Discussion}

Scientific evidence obtained from vaccine field trials has played an important role in public health, helping governments to plan successful vaccination programs. Estimating statistical power in a vaccine trial is of great importance. If done a priori (before the trial starts), the optimal sample size to be used in the trial can be estimated. The optimal sample size can give assurance that there is sufficient statistical power to detect a pre-determined vaccine effect if it does indeed exist, whilst avoiding any unnecessary waste of resources. In the current badger vaccine trial, however, the sample size was determined based on logistical issues, without potential for further expansion of the vaccine trial area. Nonetheless, power calculations were still relevant as other parameters affecting power, such as Se and $\mathrm{Sp}$ of the diagnostic test, could potentially be adjusted to optimize study power.

A simulation approach was used to estimate the power of the trial where the following assumptions were made: 
- The contact function is frequency rather than density-dependent. Riggs and Koopman $(2004,2005)$ demonstrate that sample size has a much higher impact on study power in density-dependent as compared to frequency-dependent models. This is in agreement with the results obtained in our simulations. Power remained relatively constant, varying from $90 \%$ to $93 \%$ when sample size increased from 120 to 300 badgers (assuming a $\mathrm{VE}_{\mathrm{S}}$ and $\mathrm{VE}_{\mathrm{I}}$ equal to $80 \%$ ). De Jong et al. (1995) explained how, for most animal diseases, a frequency-dependent contact rate function fits the data better than a densitydependent function. Therefore, our assumption seems reasonable.

- The prevalence of bTB in badgers in the trial area was assumed to be $30 \%$. A recent study carried out in Ireland, where comprehensive bacteriological culture methods had been used, detected a prevalence of $36.3 \%$ in badgers (Murphy et al., 2010). As highlighted previously, bTB prevalence in Ireland is known to vary in areas of high (Corner et al., 2012) and low (Murphy et al., 2011) bTB prevalence in cattle.

- The expected number of badgers being trapped in each trapping exercise was set to 120,60 and 120 for zones A, B and C, respectively. Although the three zones in the vaccine trial area were selected to have a similar number of main badger setts, the first trapping carried out during the trial revealed a lower number of badgers in Zone B compared to zones A and C.

- Data on the fraction of susceptible $(f s)$ and infected ( $f i)$ vaccinated badgers were based on expert opinion. A sensitivity analysis was carried out to see the effect on study power of changes to $f_{s}$ and $f i$. In scenarios where changes to $f_{s}$ and $f i$ were simulated, no meaningful changes were observed in study power.

- Recapture percentage was set to $50 \%$. This number was determined at the start of this study based on expert opinion from previous trapping exercises carried out in nearby areas. Subsequently, and while this study was being conducted, Byrne et al. (2012) confirmed that recapture percentage during this trial was around $50 \%$.

The most striking result obtained during the simulations was the large effect of diagnostic test specificity on study power, within the range of input values that were used for all other modelled parameters. The minimum test specificity required to achieve a power above $60 \%$ was $99.8 \%$. We were expecting low specificity values to have a substantial effect on power due to the large number of false positive badgers in a low prevalence population, however, the effect was larger than expected. This is because the vaccine effect is quickly masked by even a small proportion of false positives, randomly occurring in the many negative samples from both vaccinated and unvaccinated animals. The effect of sensitivity on power was much smaller. Assuming both $\mathrm{VE}_{\mathrm{S}}$ and $\mathrm{VE}_{\mathrm{I}}$ are equal to $80 \%$ and given a specificity of $99.8 \%$, study power remained above $50 \%$ even when test sensitivity was $40 \%$. The effect of sample size on study power was very small. This is because a small number of false 
negatives would be expected in a population were disease incidence is low, even when test sensitivity is poor. In other words, the expected number of false negatives will be a fraction of what is already a very low number. This is regardless of whether the low sensitivity is due to the low sensitivity of the diagnostic test itself or because of smaller sample size. Collectively, these results have substantial implications in terms of the optimization of the diagnostic test to be used in the vaccine trial, showing that although specificity needs to be very high, there is some degree of flexibility in terms of the sensitivity of the diagnostic test.

When the initial transmission between unvaccinated badgers was set to 0.025 , study power obtained was around $50 \%(48 \%-51 \%)$, depending on other parameters. This power would not be considered optimal for detecting a difference between the two transmission parameters $\beta_{U U}$ and $\beta_{V V}$, noting that we are seeking a study power of at least $60 \%$. Data on the transmission parameter between non-vaccinated badgers is scarce. The transmission values presented in Hardstaff et al. (2012), and referenced in this manuscript, were estimated based on studies using proximity data loggers. Logically, these are very likely an overestimation of such values, but as acknowledged by these authors, there is no data available to quantify the extent of this overestimation.

Decreasing $\mathrm{VE}_{\mathrm{S}}$ and $\mathrm{VE}_{\mathrm{I}}$ each had a negative effect on power with changes in $\mathrm{VE}_{\mathrm{S}}$ having a larger impact on power than changes in $\mathrm{VE}_{\mathrm{I}}$. For a $\beta_{U U}=0.1$, a reduction in $\mathrm{VE}_{\mathrm{I}}$ of $50 \%$ (from $80 \%$ to $40 \%$ ) had little effect on study power $(84 \%$ versus $85 \%$, respectively), while the same reduction in $\mathrm{VE}_{\mathrm{S}}$ led to a reduction in power, from $84 \%$ to $76 \%$. This difference in reduction in power can be understood from the fact that a reduction in susceptibility has a direct and full effect on the vaccinated badger. The same reduction in infectivity only has the same impact when all the infected animals that the recipient badger encounters are vaccinated. However, this was not achieved in this trial, due to the design used, and therefore the effect is diluted. Although the reduction in power due to reduction in $\mathrm{VE}_{\mathrm{s}}$ is not considered high, the effect of a low $\mathrm{VE}_{\mathrm{S}}$ on the power is nonetheless something to consider because of the uncertainty around the biological mechanisms of the BCG vaccine in badgers. Using BCG vaccine by the subcutaneous or mucosal routes in badgers, Corner et al. (2008a) demonstrated a reduction in histological lesions in vaccinated badgers compared to non-vaccinated badgers but did not show individual protection against infection. This lack of protection must be interpreted with caution as the challenging doses used during the experiments might not be representative of the natural infection dose. However, if the results obtained in the study are indicative of the real $\mathrm{VE}_{\mathrm{S}}$, study power could be somewhat compromised.

In summary, the results from our study are in line with Riggs and Koopman (2004, 2005 ) in that study power in group randomized trials depends not only on sample 
size but on many other parameters. In the current vaccine trial, power was highly dependent on the specificity of the diagnostic test and this is always to be expected when the expected incidence without vaccination is already low. For the badger vaccine trial this implies that the diagnostic test to be used has to be optimized to maximise test specificity.

\section{References}

Aznar, I., McGrath, G., Murphy, D., Corner, L.A.L., Gormley, E., Frankena, K., More, S.J., Martin, W., O'Keeffe, J., De Jong, M.C.M., 2011. Trial design to estimate the effect of vaccination on tuberculosis incidence in badgers. Vet. Microbiol. 151, 104-111.

Barth-Jones, D.C., Adams, A.L., Koopman, J.S., 2004. Monte Carlo simulation experiments for analysis of HIV vaccine effects and vaccine trial design. Proceedings of the 32nd conference on Winter simulation. Society for Computer Simulation International, Orlando, Florida.

Byrne, A.W., O'Keeffe, J.J., Green, S., Sleeman, D.P., Corner, L.A.L., Gormley, E., Murphy, D., Martin, S.W., Davenport, J., 2012. Population estimation and trappability of the European badger (Meles meles): Implications for tuberculosis management. PLoS One 7, e50807.

Charvat, B., Brookmeyer, R., Herson, J., 2009. The effects of herd immunity on the power of vaccine trials. Stat. Biopharm. Res. 1, 108-117.

Corner, L.A., Costello, E., Lesellier, S., O'Meara, D., Gormley, E., 2008 a. Vaccination of European badgers (Meles meles) with BCG by the subcutaneous and mucosal routes induces protective immunity against endobronchial challenge with Mycobacterium bovis. Tuberculosis (Edinburgh, Scotland) 88, 601-609.

Corner, L.A.L., Costello, E., Lesellier, S., O'Meara, D., Gormley, E., 2008b. Experimental tuberculosis in the European badger (Meles meles) after endobronchial inoculation with Mycobacterium bovis: II. Progression of infection. Res. Vet. Sci. 85, 481-490.

Corner, L.A.L., Costello, E., Lesellier, S., O'Meara, D., Sleeman, D.P., Gormley, E., 2007. Experimental tuberculosis in the European badger (Meles meles) after endobronchial inoculation of Mycobacterium bovis: I. Pathology and bacteriology. Res. Vet. Sci. 83, 53-62.

Corner, L.A.L., Costello, E., O'Meara, D., Lesellier, S., Aldwell, F.E., Singh, M., Hewinson, R.G., Chambers, M.A., Gormley, E., 2010. Oral vaccination of badgers (Meles meles) with BCG and protective immunity against endobronchial challenge with Mycobacterium bovis. Vaccine 28, 6265-6272.

Corner, L.A.L., O’Meara, D., Costello, E., Lesellier, S., Gormley, E., 2012. The distribution of Mycobacterium bovis infection in naturally infected badgers. Vet. J. 194, 166-172.

De Jong, M.C.M., Diekmann, O., Heesterbeek, H., 1995. How does transmission of infection depend on population size?. In: Mollison, D., Epidemic models: their 
structure and relation to data. Cambridge: Cambridge University Press, pp. 8494.

Halloran, M.E., Longini, I.M., Jr, Struchiner, C.J., 1999. Design and interpretation of vaccine field studies. Epidemiol. Rev. 21, 73-88.

Hardstaff, J.L., Bulling, M.T., Marion, G., Hutchings, M.R., White, P.C.L., 2012. Impact of external sources of infection on the dynamics of bovine tuberculosis in modelled badger populations. BMC Vet. Res. 8, 92.

Hardstaff, J.L., Bulling, M.T., Marion, G., Hutchings, M.R., White, P.C.L., 2013. Modelling the impact of vaccination on tuberculosis in badgers. Epidemiol. Infect. doi:10.1017/S0950268813000642.

Hayes, R.J., Alexander, N.D., Bennett, S., Cousens, S.N., 2000. Design and analysis issues in cluster-randomized trials of interventions against infectious diseases. Stat. Methods Med. Res. 9, 95-116.

Lesellier, S., Corner, L., Costello, E., Lyashchenko, K., Greenwald, R., Esfandiari, J., Singh, M., Hewinson, R.G., Chambers, M., Gormley, E., 2009. Immunological responses and protective immunity in BCG vaccinated badgers following endobronchial infection with Mycobacterium bovis. Vaccine 27, 402-409.

Longini, I.M., Jr, Sagatelian, K., Rida, W.N., Halloran, M.E., 1998. Optimal vaccine trial design when estimating vaccine efficacy for susceptibility and infectiousness from multiple populations. Stat. Med. 17, 1121-1136.

More, S.J., 2009. What is needed to eradicate bovine tuberculosis successfully: an Irish perspective. Vet. J. 180, 275-278.

Murphy, D., Gormley, E., Collins, D.M., McGrath, G.E., Sovsic, E., Costello, E., Corner, L.A.L., 2011. Tuberculosis in cattle herds are sentinels for Mycobacterium bovis infection in European badgers (Meles meles): The Irish Greenfield Study. Vet. Microbiol. 151, 120-125.

Murphy, D., Gormley, E., Costello, E., O'Meara, D., Corner L.A.L., 2010. The prevalence and distribution of Mycobacterium bovis infection in European badgers (Meles meles) as determined by enhanced post mortem examination and bacteriological culture. Res. Vet. Sci. 88, 1-5.

Riggs, T.W., Koopman, J.S., 2004. A stochastic model of vaccine trials for endemic infections using group randomization. Epidemiol. Infect. 132, 927-938.

Riggs, T., Koopman, J.S., 2005. Maximizing statistical power in group-randomized vaccine trials. Epidemiol. Infect. 133, 993-1008.

Sheridan, M., 2011. Progress in tuberculosis eradication in Ireland. Vet. Microbiol. $151,160-169$.

Walters, S.J., 2004. Sample size and power estimation for studies with health related quality of life outcomes: a comparison of four methods using the SF-36. Health Qual. Life Outcomes 2, 26. 
Estimating the power of a Mycobacterium bovis vaccine trial in Irish badgers Supplementary material

$\underline{\text { Appendix A }}$

*SAS (9.2) program to evaluate study power of a badger vaccine trial;

*For running under SAS 9.1, 'LBetaEstimate' should be replaced by 'estimate';

*Published in Preventive Veterinary Medicine: volume, issue, pages;

options nocenter;

libname trial 'd: \power_sas';

*Reading basic data;

data fixed0;

input period area vaccinated fs fi prev total_n;

cards;

$\begin{array}{lllllll}2 & 1 & 0 & 0 & 0 & 0.3 & 120 \\ 3 & 1 & 0 & 0 & 0 & 0.3 & 120 \\ 4 & 1 & 0 & 0 & 0 & 0.3 & 120 \\ 5 & 1 & 0 & 0 & 0 & 0.3 & 120 \\ 6 & 1 & 0 & 0 & 0 & 0.3 & 120 \\ 7 & 1 & 0 & 0 & 0 & 0.3 & 120 \\ 2 & 2 & 0 & 0.30 .3 & 0.3 & 60 \\ 3 & 2 & 0 & 0.30 .3 & 0.3 & 60 \\ 4 & 2 & 0 & 0.30 .3 & 0.3 & 60 \\ 5 & 2 & 0 & 0.40 .4 & 0.3 & 60 \\ 6 & 2 & 0 & 0.40 .4 & 0.3 & 60 \\ 7 & 2 & 0 & 0.40 .4 & 0.2 & 60 \\ 2 & 3 & 0 & 0.60 .6 & 0.2 & 120 \\ 3 & 3 & 0 & 0.60 .6 & 0.2 & 120 \\ 4 & 3 & 0 & 0.60 .6 & 0.1 & 120 \\ 5 & 3 & 0 & 0.70 .7 & 0.1 & 120 \\ 6 & 3 & 0 & 0.80 .8 & 0.1 & 120 \\ 7 & 3 & 0 & 0.80 .8 & 0.05 & 120 \\ 2 & 1 & 1 & 0 & 0 & 0.3 & 120 \\ 3 & 1 & 1 & 0 & 0 & 0.3 & 120 \\ 4 & 1 & 1 & 0 & 0 & 0.3 & 120 \\ 5 & 1 & 1 & 0 & 0 & 0.3 & 120 \\ 6 & 1 & 1 & 0 & 0 & 0.3 & 120 \\ 7 & 1 & 1 & 0 & 0 & 0.3 & 120 \\ 2 & 2 & 1 & 0.30 .3 & 0.3 & 60 \\ 3 & 2 & 1 & 0.30 .3 & 0.3 & 60\end{array}$




$\begin{array}{llllll}4 & 2 & 1 & 0.30 .3 & 0.3 & 60 \\ 5 & 2 & 1 & 0.40 .4 & 0.3 & 60 \\ 6 & 2 & 1 & 0.40 .4 & 0.3 & 60 \\ 7 & 2 & 1 & 0.40 .4 & 0.2 & 60 \\ 2 & 3 & 1 & 0.60 .6 & 0.2 & 120 \\ 3 & 3 & 1 & 0.60 .6 & 0.2 & 120 \\ 4 & 3 & 1 & 0.60 .6 & 0.1 & 120 \\ 5 & 3 & 1 & 0.70 .7 & 0.1 & 120 \\ 6 & 3 & 1 & 0.80 .8 & 0.1 & 120 \\ 7 & 3 & 1 & 0.80 .8 & 0.05 & 120\end{array}$

;

data fixed; set fixed0;

*Set sample size as retrapping percentage;

trapperc $=100$; total_n=int(total_n*trapperc/100);

run;

*Run (or block) next 2 lines to suppress (or not) screen output;

proc printto log='d: \power_sas $\backslash$ log.txt' new;run;

proc printto print='d:|power_saslout.txt' new;run;

*Call macro doloop;

\%doloop; quit;

*Back to screen output;

proc printto;run;

*MACRO DESCRIPTIONS ARE BELOW;

*Macro DOLOOP evaluates power at varying values of SE and SP;

$\%$ macro doloop;

*First clean directory from existing files in case macro doloop is re-run;

$\%$ delfile(trial.collect6);

\%delfile(trial.collectC);

*Set range of SE and SP, multiplied with 10,000 in order to allow small steps;

\%do SE $=3000 \%$ to $4000 \%$ by 100 ;

\%do SP $=9800 \%$ to $9900 \%$ by 2 ;

*Call macro simulate (within macro doloop);

$\%$ simulate

data trial.collect2; set trial.collect; 
*Keep the records with exponentiated coefficients;

if label in ("Exp(vacc=0)","Exp(vacc=1)");

run;

*Put coeffs and stderrs of each dataset at one line;

data trial.collect3; set trial.collect $2 ; \mathrm{k} 1=$ round $\left(\_\mathrm{N} \_/ 2\right) ; \mathrm{kse}=\& \mathrm{SE} ; \mathrm{ksp}=\& \mathrm{SP}$;

run;

proc transpose data $=$ trial.collect 3 out $=$ trial.collect $4 a$ prefix $=$ est_;var LBetaEstimate ; by k1 kse ksp ;run;

proc transpose data $=$ trial.collect 3 out $=$ trial.collect $4 \mathrm{~b}$ prefix $=$ stderr_;var stderr;by

k1 kse ksp;run;

proc transpose data $=$ trial.collect 3 out $=$ trial.collect $4 \mathrm{c}$ prefix $=\mathrm{qc} 0$;;var qc0; by k1 kse ksp;run;

proc transpose data=trial.collect 3 out=trial.collect 4 d prefix=qc1_;var qc1; by k1 kse ksp;run;

data trial.collect5;

merge trial.collect4a trial.collect $4 \mathrm{~b}$ trial.collect $4 \mathrm{c}$ trial.collect $4 \mathrm{~d}$;

by k1 kse ksp;

*Performing paired T-test using estimates and standard errors;

$\mathrm{n} 1=21 ; \mathrm{n} 2=14$

$\mathrm{C} 1=($ stderr $1 * * 2) / \mathrm{n} 1 ; \mathrm{C} 2=($ stderr $2 * * 2) / \mathrm{n} 2$;

tvalue $=($ est 1 -est 2$) /$ sqrt $(\mathrm{C} 1+\mathrm{C} 2)$;

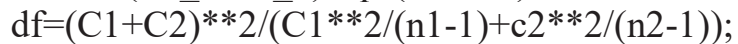

if tvalue $>0$ then $\mathrm{p}=1-\mathrm{CDF}($ 'T',tvalue,df);else $\mathrm{p}=1$;

if tvalue $=$. then $\mathrm{p}=$;

if $\mathrm{p}<0.05$ then test $=1$; else test $=0$;

if tvalue $=$. then test $=$;

*Variable test indicates whether $\mathrm{p}$ is smaller than 0.05 ;

keep k1 kse ksp est_1 est_2 stderr_1 stderr_2 tvalue df p test qc0_1 qc1_2 ;

run;

*The power is calculated and the average fraction $\mathrm{C} / \mathrm{NCS}$ for the vaccinates and the non-vaccinates for each combination of SE and SP;

*Results are stored in file trial.collect6;

proc means data $=$ trial.collect5 $\mathrm{n}$ mean noprint ; var test; output out=powerfile

mean=power $\mathrm{n}=$ count; by kse ksp;run;

proc means data=trial.collectC $\mathrm{n}$ mean noprint; var fraction; output

out $=$ fractionfile 0 mean $=$ mfrac_notvacc; by kse ksp; where vaccinated $=0$;run;

proc means data $=$ trial.collectC $\mathrm{n}$ mean noprint; var fraction; output

out=fractionfile1 mean=mfrac_vacc; by kse ksp;where vaccinated=1;run; 
data combine; merge powerfile fractionfile 0 fractionfile1; by kse ksp; drop _type freq_; run;

proc append base $=$ trial.collect 6 data $=$ combine;run;

$\%$ end;\%end;

$\%$ mend doloop;

*Macro simulate creates and analyses datasets;

\%macro simulate;

$\%$ delfile(trial.collect);

\%delfile(trial.collectC);

*Set number of simulations, run time heavily depends on this;

\%do $\mathrm{j}=1 \%$ to 1000 ;

*data trial.fixed\&j;

data fixed 2 ; Contains the created dataset;

set fixed; *Reads the initial dataset;

*Set initial transmission parameters;

$\mathrm{uu}=0.1 ; \mathrm{vu}=0.05 ; \mathrm{uv}=0.05 ; \mathrm{vv}=\mathrm{vu} * \mathrm{uv} ;$ OFFSET $=\log (\mathrm{prev}) ;$

*NCS=number caught susceptible;

if vaccinated eq 0 then do; $\mathrm{NCS} 0=(1-\mathrm{fs}) *$ total $\mathrm{n} *(1-\mathrm{prev})$; $\mathrm{NCS}=$ round(NCS0);end;

if vaccinated ne 0 then do; $\mathrm{NCS} 0=$ total_n*(1-prev) $* \mathrm{fs} ; \mathrm{NCS}=$ round(NCS0);end;

*prob=probability of being infected when vaccinated or not;

if vaccinated eq 0 then $\operatorname{prob}=1-\exp (-1 *(\mathrm{uu} *(1-\mathrm{fi})+\mathrm{vu} * \mathrm{fi}) * \mathrm{prev})$;

if vaccinated ne 0 then $\operatorname{prob}=1-\exp (-1 *(\mathrm{uv} *(1-\mathrm{fi})+\mathrm{vv} * \mathrm{fi}) * \mathrm{prev})$;

*Number infected (C);

*Prepare for macro critbinom, alpha is drawn from a Uniform distribution; alpha $=$ ranuni $(0)$; trials $=$ NCS; $p_{-}$success $=$prob;

*Call macro critbinom to obtain the critical value based on alpha, NCS and prob;

$\%$ critbinom(p_success $=$ prob)

$\mathrm{C}=\mathrm{NCS}-($ critval);

$\mathrm{k}=\& \mathrm{j}$;

*Number test positives depend on NCS, C, SE and SP;

*Call macro critbinom to obtain the critical value based on alpha, $\mathrm{C}$ and $\mathrm{p} 1$;

alpha $=$ ranuni $(0) ;$ trials $=\mathrm{C} ; \mathrm{p} 1=\& \mathrm{SE} / 10000 ;$

$\%$ critbinom(p_success $=\mathrm{p} 1$ ) 
*True positives;

$\mathrm{TP}=\mathrm{C}-($ critval);

$\mathrm{k}=\& \mathrm{j}$;

*Call macro critbinom to obtain the critical value based on alpha, $\mathrm{C}$ and $\mathrm{p} 2$;

alpha $=$ ranuni $(0)$; trials $=$ NCS-C; $22=(10000-\& S P) / 10000$;

$\%$ critbinom $\left(\mathrm{p} \_\right.$success $=\mathrm{p} 2$ )

*False positives;

$\mathrm{FP}=(\mathrm{NCS}-\mathrm{C})-($ critval);

$\mathrm{k}=\& \mathrm{j}$;

$\mathrm{kSE}=\& \mathrm{SE}$

$\mathrm{kSP}=\& \mathrm{SP}$;

*Total number tested positive;

observed $=\mathrm{TP}+\mathrm{FP}$;

run;

*Fraction of cases/trial is calculated and stored in collectC;

data keepC; set FIXED2; if NCS gt 0 then fraction=C/NCS; keep k kse ksp vaccinated NCS C critval TP FP observed fraction;run;

proc append base $=$ trial. $\operatorname{collectC}$ data $=\mathrm{keepC}$; run;

*Next the created dataset is analysed and output saved, separately for the vaccinated and unvaccinated records;

*Proc genmod is used because it has the cll link function available;

\%delfile(run0);

ods output estimates $=$ run $0(\mathrm{keep}=\mathrm{k}$ label LBetaEstimate stderr);

*proc genmod data=trial.fixed $\&$ j;

proc genmod data $=$ fixed 2 ; where ncs gt 0 and vaccinated $=0$;

model observed $/ \mathrm{ncs}=\mathrm{fi} / \mathrm{dist}=$ binomial link $=$ cll offset $=$ offset;

by k kse ksp; ${ }^{*} \mathrm{k}$ is just a count variable;

estimate 'vacc $=0$ ' intercept 1 fi $0 / e$ exp;

ODS select estimates;

run;

\%append0(run0);

\%delfile(run1); 
ods output estimates=run1 $(\mathrm{keep}=\mathrm{k}$ label LBetaEstimate stderr);

*proc genmod data $=$ trial.fixed $\&$;

proc genmod data $=$ fixed 2 ; where ncs gt 0 and vaccinated $=1$;

model observed $/$ ncs $=$ fi $/$ dist $=$ binomial link $=$ cll offset $=$ offset;

by k kse ksp;

estimate 'vacc $=1$ ' intercept 1 fi $1 / \mathrm{e} \exp$;

ODS select estimates;

run;

\%append1(run1);

\%end;

* Show screen output after one simulation of 1000 iterations;

proc printto;run;

\%put \&se \&sp;

*Overwrite the output files to prevent they grow very large;

proc printto log='d: power_sas $\backslash$ log.txt' new;run;

proc printto print='d:|power_sas $\backslash$ lout.txt' new;run;

$\%$ mend simulate;

*SAS does not have the Excel CRITBINOMIAL (BIN.INV) function, but a macro can be derived from

http://www.listserv.uga.edu/cgi-bin/wa?A2=ind0212c\&L=sas-1\&P=27308;

$\%$ macro critbinom $($ critval $=$ critval,

trials=trials,

p_success $=p$ _success,

alpha $=$ alpha

);

\footnotetext{
$/ * * * * * * * * * * * * * * * * * * * * * * * * * * * * * * * * * * * * * * * * * * * * * * * * * * * * * * * * * /$

/* Macro variables name datastep variables: */

/* */

/* CRITVAL: Variable holding returned value $*$ /

/* TRIALS: Number of independent bernoulli trials */

/* P_SUCCESS: Success probability per bernoulli trial */

/* ALPHA: Criterion value with $0<$ alpha $<1 \quad * /$

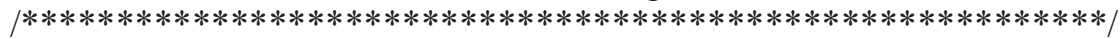


if nmiss(\&trials, \&p_success, \&alpha) $>0$ then do;

put "Error: Invocation of CRITBINOM subroutine -";

put " Invalid specification of TRIALS, P_SUCCESS, or ALPHA";

end;

else do;

\&critval=int (\&trials/2);

max $=\&$ trials;

$\min =0$;

do until(_test $1>\&$ alpha \&_test $2<\&$ alpha);

test1 $=$ cdf('BINOM', \& trials-\&critval, \&p_success, \&trials);

test2 = cdf('BINOM', \& trials-(\&critval+1), \&p_success, \&trials);

if_test $1<\&$ alpha then do;

max $=\&$ critval;

\&critval=int $((\&$ critval__min $) / 2)+\_\min ;$

end;

else if _test $2>\&$ alpha then do;

min $=$ \&critval;

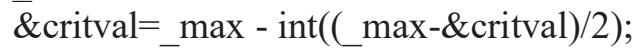

end;

end;

end;

drop_min_max_test1_test2;

\%mend;

*Macro DELFILE deletes specified files;

$\%$ macro delfile (DS);

\%if \%sysfunc (exist (\&DS)) \%then \%do;

proc sql noprint; drop table \&DS; quit;

$\%$ end;

$\%$ mend delfile;

*Macro's APPEND0 EN APPEND1 accumulate genmod outcomes;

*In case the original genmod halted because of non-convergence, results of an intercept only model are stored instead;

$\%$ macro append0(R0);

\%if \%sysfunc (exist (\&R0)) \%then \%do;

data run 0 ;set run $0 ; \mathrm{qc} 0=0 ; \mathrm{qc} 1=. ; \mathrm{run}$;

proc append base $=$ trial. collect data $=$ run0; run;

$\%$ end;

$\%$ else \%do;

ods output estimates $=$ run $0(\mathrm{keep}=\mathrm{k}$ label LBetaEstimate stderr $)$;

*proc genmod data=trial.fixed\&j; 
proc genmod data $=$ fixed 2 ; where ncs gt 0 and vaccinated $=0$; model observed $/ \mathrm{ncs}=/$ dist $=$ binomial link $=$ cll offset $=$ offset; by k kse ksp;*k is just a count variable;

estimate 'vacc $=0$ ' intercept $1 / \mathrm{e}$ exp;

ODS select estimates;

run;

data run $0 ;$ set run $0 ; \mathrm{qc} 0=1 ; \mathrm{qc} 1=. ;$ run;

proc append base $=$ trial. collect data $=$ run 0 ; run;

$\%$ end;

\%mend append0;

\%macro append1(R1);

\%if \%sysfunc (exist (\&R1)) \%then \%do;

data run $1 ;$ set run $1 ; \mathrm{qc} 1=0 ; \mathrm{qc} 0=. ;$ run;

proc append base $=$ trial.collect data $=$ run 1 ; run;

$\%$ end;

$\%$ else \%do;

ods output estimates=run1 ( $k$ eep=k label LBetaEstimate stderr);

*proc genmod data=trial.fixed\&j;

proc genmod data $=$ fixed2; where ncs gt 0 and vaccinated $=1$;

model observed $/ \mathrm{ncs}=/$ dist $=$ binomial link $=$ cll offset $=$ offset;

by k kse ksp;*k is just a count variable;

estimate 'vacc=1' intercept 1 /e exp;

ODS select estimates;

run;

data run $1 ;$ set run $1 ; \mathrm{qc} 1=1 ; \mathrm{qc} 0=. ;$ run;

proc append base $=$ trial.collect data $=$ run 1 ; run;

$\%$ end;

\%mend append1; 

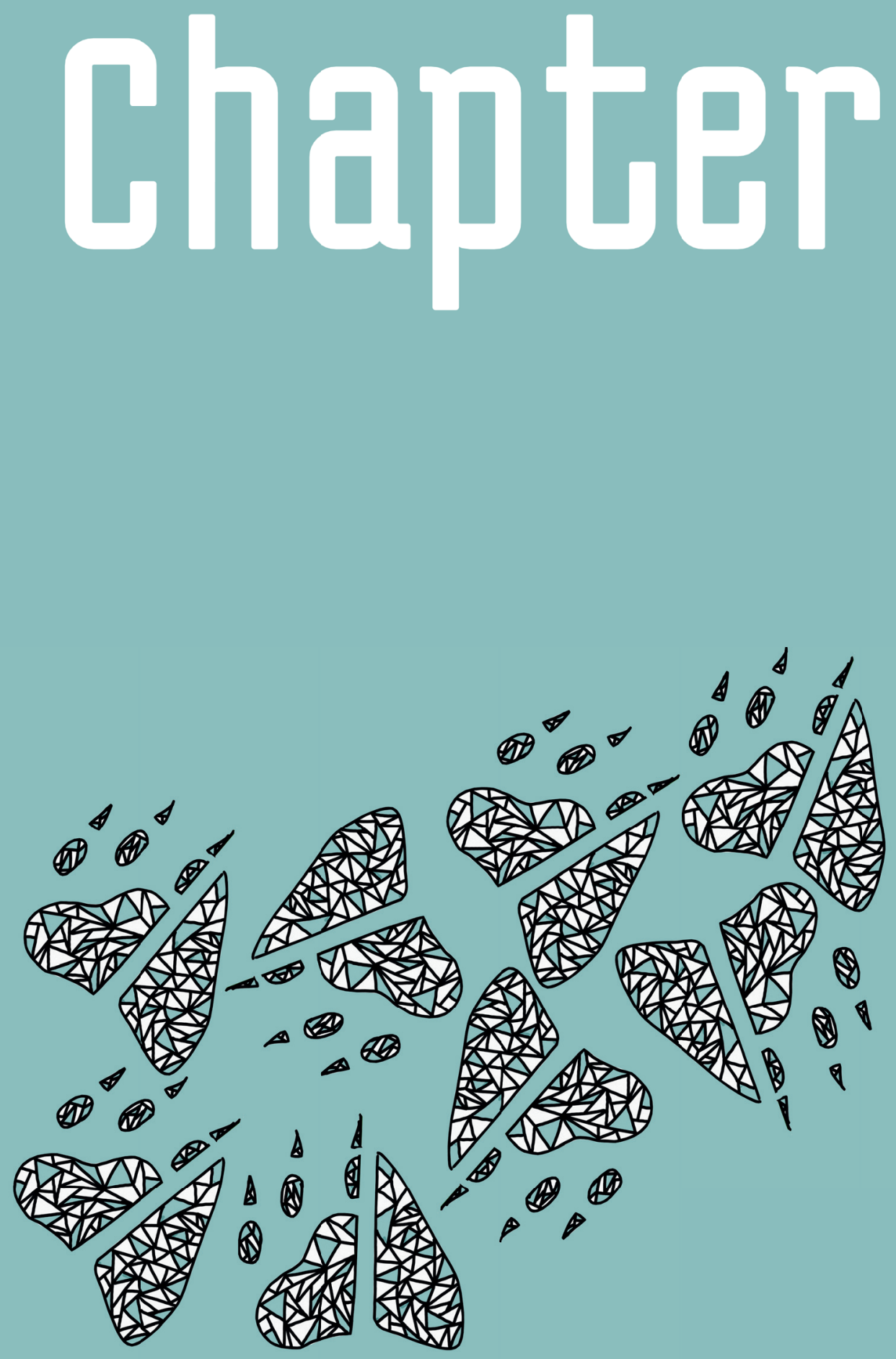


\section{optimising and evaluating the characteristics of a multiple antigen ELISH for detection of Mycobacterium bovis infection in a badger vaccine field trial}

Aznar, I. , Frankena, K. , More, s.J. , Whelan, C. , Martin, W. , Gormley, \&. , Corner, L.A.L., Murphy, D. , de Jong, M.C.M. 
Chapter 4

\section{Optimising and evaluating the characteristics of a multiple antigen ELISA for detection of Mycobacterium bovis infection in a badger vaccine field trial}

Aznar, I., Frankena, K., More, S.J., Whelan, C., Martin, W., Gormley, E., Corner, L.A.L., Murphy, D., de Jong, M.C.M.

PLoS One 9, e100139

(C) 2014 Aznar et al. This is an open-access article distributed under the terms of the Creative Commons Attribution License, which permits unrestricted use, distribution, and reproduction in any medium, provided the original author and source are credited. 


\begin{abstract}
A long-term research programme has been underway in Ireland to evaluate the usefulness of badger vaccination as part of the national bTB (bovine tuberculosis) control strategy. This culminated in a field trial which commenced in county Kilkenny in 2009 to determine the effects of badger vaccination on Mycobacterium bovis transmission in badgers under field conditions. In the present study, we sought to optimise the characteristics of a multiplex chemiluminescent assay for detection of $M$. bovis infection in live badgers. Our goal was to maximise specificity, and therefore statistical power, during evaluation of the badger vaccine trial data. In addition, we also aimed to explore the effects of vaccination on test characteristics. For the test optimisation, we ran a stepwise logistic regression with analytical weights on the converted Relative Light Units (RLU) obtained from testing blood samples from 215 badgers captured as part of culling operations by the national Department of Agriculture, Food and the Marine (DAFM). The optimised test was applied to two other datasets obtained from two captive badger studies (Study 1 and Study 2), and the sensitivity and specificity of the test was attained separately for vaccinated and non-vaccinated badgers. During optimisation, test sensitivity was maximised (30.77\%), while retaining specificity at $99.99 \%$. When the optimised test was then applied to the captive badger studies data, we observed that test characteristics did not vary greatly between vaccinated and non-vaccinated badgers. However, a different time lag between infection and a positive test result was observed in vaccinated and non-vaccinated badgers. We propose that the optimized multiplex immunoassay be used to analyse the vaccine trial data. In relation to the difference in the time lag observed for vaccinated and non-vaccinated badgers, we also present a strategy to enable the test to be used during trial evaluation.
\end{abstract}




\section{Introduction}

Badgers play an important role in the epidemiology of bovine tuberculosis (bTB) in Ireland, by acting as a source of infection to cattle [1,2]. The prevalence of Mycobacterium bovis infection in badgers, based on animals captured as part of culling operations by the national Department of Agriculture, Food and the Marine (DAFM), was estimated recently at 36.3\% [3], although this is known to vary substantially between areas where bTB in cattle is problematic [4] or absent [5]. Sustainable progress towards eradication of $M$. bovis infection in cattle might not be possible in the face of continued spillover of infection from badgers to cattle [2].

Several control options are available to limit transmission of infection from badgers to cattle, including reducing the frequency of contact between these species and decreasing the proportion of the badger population susceptible to infection, through vaccination [2]. In Ireland, focused badger culling is being used to reduce contact rates between badgers and cattle in areas of high bTB incidence in cattle. However, it is hoped that badger culling can be replaced by, or supplemented with, badger vaccination. A long-term Irish research programme is on-going to evaluate the usefulness of badger vaccination as part of the national bTB control strategy. A series of pen-based vaccination trials have been conducted, where badgers were vaccinated with Bacillus Calmette-Guerin (BCG) and subsequently challenged with M. bovis, and the impact of vaccination on pathology, bacteriology and progression of infection in badgers has previously been reported [6-9]. Subsequent to this work, a field trial commenced in county Kilkenny, in 2009, to determine the effects of badger vaccination on $M$. bovis transmission in badgers under field conditions [10]. The field trial design will enable comparison of bTB incidence between vaccinated and unvaccinated badgers in three areas of differing vaccine coverage (100, 50 and $0 \%)$.

A number of challenges have been encountered during the design of the field trial in Co. Kilkenny, including: a) the need to fully understand the biology underpinning protection following BCG vaccination, both in individual badgers and within the broader badger population (including the likelihood of reduction of infectiousness and therefore transmission); b) the need to identify the infection status of each badger at each capture event (a capture/ recapture design has been employed), and c) the need for sufficient statistical power in the aforementioned design. There is now a better understanding of options to address the first and third of these challenges $[10,11]$. In this paper, we consider the second of these challenges, that is, the need for a test to identify the infection status of individual badgers at each capture event. It has been shown recently that this diagnostic test will need a specificity very close to $100 \%$ in order to obtain sufficient study power [11]. The authors estimated a minimum specificity of $99.8 \%$ to achieve a power above $60 \%$ in this trial. The need 
for a high specificity reflects the fact that the cost of false positive test results is much higher than that of false negative results.

A significant amount of work in relation to diagnostic methods for tuberculosis in live badgers has been conducted in Ireland and the United Kingdom [12-17]. Assays based on the measurement of cellular responses, such as gamma-interferon, have attracted considerable interest as they are expected to deliver a higher sensitivity in comparison to antibody-based assays [13, 15]. Furthermore, these cell response assays have the advantage of being able to detect earlier stages of infection [18]. However, one of the drawbacks of these assays is the large effect of pre-culture holding time and temperature on gamma-interferon responses [19,20]. Several other bTB assays have been developed. The Brock test is an indirect ELISA that measures M. bovis-specific antibody responses to a single antigen, MPB83 [21,22]. MPB83 is expressed by other members of M. tuberculosis complex, but is serodominant in M.bovis infection. Subsequent studies have shown that test sensitivity and specificity can be enhanced by using a mixture of antigens rather than a single antigen. Based on the use of a multi-antigen print immunoassay (MAPIA) and culture as the gold standard, the sensitivity was found to increase from $47.4 \%$ to $52.6 \%$ and the specificity from $89 \%$ to $95 \%$ [23]. In an attempt to simplify the procedure, thereby allowing badger testing to be performed in the field, a lateral flow immunoassay (the Brock TB Stat-Pak assay; Chembio Diagnostic Systems, Inc., Medford, NY) was developed $[14,16]$.

Here, we will look at the Enfer chemiluminescent multiplex ELISA system, originally developed for testing $M$. bovis in cattle [24,25]. The test was adapted for badgers and applied to 200 blood samples from badgers captured in Ireland in areas of high bovine tuberculosis prevalence [26], this study reported a sensitivity and specificity in badgers of $56.7 \%$ and $96.99 \%$, respectively, when using a panel of $M$. bovis antigens. The availability of this test, the fact that this test can be performed in stored blood samples without losing sensitivity or specificity, and its quantitative nature, made this test the test of choice. Given this background, the current study had two objectives. First, we sought to statistically optimise a multiplex chemiluminescent assay for detection of $M$. bovis infection in live badgers to maximise specificity, and therefore statistical power, during evaluation of the badger vaccine trial in Ireland. Second, we aimed to explore the effects of vaccination on test characteristics and to review the implications for analysis of the data from the Kilkenny badger vaccine trial.

\section{Material and Methods}

The 215 blood samples used for test optimization were part of an archive. The badgers from which these samples had been taken, had been captured as part of the 
DAFM culling operations carried out in the Republic of Ireland. The Department of Arts, Heritage and the Gaeltacht, specifically the National Parks and Wildlife Service, issues licences to the Department of Agriculture, Food and the Marine to undertake the capturing programme. The captive badger studies were carried out under license (No. B100/3187, Cruelty to Animals Act 1876) issued by the Department of Health and Children, and ethical approval was obtained from the UCD Animal Research Ethics Committee (AREC-P-04-28 and AREC-P-24-06).

\section{$\underline{1 .}$ Test optimisation using naturally infected wild badgers}

\subsection{Samples}

Serum samples from 215 badgers captured as part of the DAFM culling operations carried out in the Republic of Ireland were used for optimisation of the diagnostic test. Focused (reactive) badger removal is conducted under license in the vicinity of herds that have had a severe bTB breakdown where the cause cannot be attributed to non-wildlife sources. The serum samples had been collected for archiving purposes. A study by Murphy et al. (2010) [3] looked at these badgers for gross visible lesions of TB at post mortem and samples were collected from a range of tissues and pooled into groups for bacterial culture of M. bovis. An aseptic technique was used when preparing tissue samples to minimize contamination before culture. In the current study, culture results were used as the gold standard and a badger was considered infected when M. bovis was isolated from any of the samples taken.

\subsection{Multiplex immunoassay}

Antibody responses, expressed as relative light units (RLU), to a panel of antigens were measured using the Enfer chemiluminescent multiplex ELISA system (Enfer Scientific, Co. Kildare, Ireland). The antigen panel consisted of the following six recombinant proteins: MPB83, MPB70, Rv3616c fragment and full protein, ESAT6 and CFP10, as well as purified protein derivative from M. bovis (PPDb) and a peptide of MPB70. Tests were carried out by Enfer Scientific using 96-well microtitreplates. Recombinant antigens (Fusion Antibodies Ltd. (Belfast)) and peptides (Genosphere Biotechnologies (France)) were prepared as previously described by Whelan et al. (2008)[24].

The multiplex assay was carried according to Whelan et al. (2008) [24]. Serum samples were diluted 1:450 into sample dilution buffer and mixed. A 50 $\mu 1$ sample dilution was added per well. The plates were incubated at room temperature with agitation for 90 minutes. The plates were washed once with Enfer Wash Buffer (Enfer Scientific) and aspirated. The detection antibody (CF2/HRPo Anti-Badger IgG-HRP conjugate, kindly provided by Mark Chambers, AHVLA, Weybridge, UK) 
was prepared to a dilution of 1:75,000 in detection antibody dilution buffer. After addition of $50 \mu 1$ of the detection antibody to each test well, the plates were incubated at room temperature for 30 minutes with agitation. The plates were washed as above and $50 \mu 1$ of chemiluminescent substrate (50:50 substrate and diluent) was added per well. Signals were captured and data were extracted and analysed as previously described [24].

\subsection{Data analyses}

The 8 antibody response RLU-signals were blank-corrected by subtracting a blank spot signal specific to each sample. Initially, all zero or negative test-result values were converted to 0.0001 to allow for logarithmic transformation; however, the logarithmic transformation did not improve ROC curves. The blank-corrected values with negative values converted to 0.0001 will be referred to as "converted RLU". Descriptive statistics (mean, standard deviation, minimum, maximum and median) of the converted RLU to the 8 antigens were calculated separately for non infected (NI) and infected (I) badgers. ROC curves for each antigen were constructed, and the ROC curve showing the largest sensitivity, with specificity set at $99.99 \%$, is presented in this manuscript.

A stepwise logistic regression, with culture status as the dependent variable, the converted RLU to each of the 8 antigens as the independent variables, and using a significance level of 0.05 , was carried out. Analytical weights were used to account for the cost of false positive test results being higher than that of negative test results. After exploring different cost ratios, a cost ratio of 100:1 (false positive: false negative) was selected. Cost ratios higher than 100:1 did not improve the ROC curve. From the logit obtained after using logistic regression, a cut off was chosen that allowed sensitivity to be maximised for specificity equal to $99.99 \%$. A HosmerLemeshow test was used to assess the goodness of fit of the final model. For the best linear combination of antigens, a ROC curve was created. All analyses were performed using Stata version 12 (Stata Corp, College Station, TX, USA).

2. Evaluation of test characteristics in vaccinated and non-vaccinated captive badgers

\subsection{Study 1 samples}

Serum samples were available from a laboratory vaccine trial (Study 1). This trial was conducted to compare the levels of protection between vaccinated and nonvaccinated badgers, and between badgers vaccinated with different vaccines. Briefly, badgers were sourced from an area free of bTB and allowed to adapt to captivity for 12 weeks prior to the start of the experiment. During adaptation, the badgers were screened for tuberculosis using a lymphocyte transformation assay (LTA). The 
experiment consisted of three groups of badgers: animals vaccinated $\left(10^{8} \mathrm{CFU}\right)$ with either BCG Danish 1331 ( $\mathrm{n}=8$ animals) or BCG Pasteur 1173P2 (n=7), and controls $(n=8)$. All badgers were challenged by the endo-bronchial route with $6 \times 10^{3} \mathrm{CFU} M$. bovis. The badgers were euthanised 15 weeks post-challenge and subjected to a detailed post-mortem examination. Blood samples were taken twice a month prior to vaccination (6 samples per badger) and once a month subsequently ( 2 samples prior and 4 samples subsequent to challenge, per badger).

\subsection{Study 2 samples}

Serum samples were also available from a second captive badger study (Study 2). Data were available from a group of 9 badgers that were vaccinated $\left(10^{8} \mathrm{CFU}, \mathrm{BCG}\right.$ Danish strain) and a group of 10 badgers that served as a control group. Badgers were challenged with $3 \times 10^{2} \mathrm{CFU}$ of $M$. bovis by the endo-bronchial route, and followed for 51 weeks after challenge. Blood samples were taken every two weeks before badgers were vaccinated ( 3 samples per badger) and once a month subsequent to vaccination ( 3 samples before and 10 samples after challenge per badger) with a further sample taken before badgers were euthanized three months later.

\subsection{Multiplex immunoassay}

The assay was conducted as described previously.

\subsection{Data analysis}

For each of the captive studies, descriptive statistics of the antibody responses to each of the 8 antigens were calculated independently for each of the following badger categories: non-vaccinated - non-infected (Category 1), vaccinated - non-infected (Category 2), non-vaccinated - infected (Category 3) and vaccinated - infected (Category 4). In Study 1, the descriptive analysis was done taking into account only those badgers vaccinated with the Danish strain and then repeated using data from both groups of vaccinated badgers (Danish and Pasteur).

The optimal antigen combination (described in section 2.1) was applied to the data from Study 1 and Study 2 resulting in estimated logits; by applying the selected cutoff to the logits obtained, the sensitivity and specificity of the multiplex immunoassay test was estimated separately for each of the studies. In order to be consistent, only samples from badgers vaccinated with the Danish strain were used for estimating sensitivity and specificity in Study 1.The sensitivity and specificity was also estimated separately for vaccinated and non-vaccinated badgers in Study 2. Badgers in these datasets were considered infected one day after they had been challenged. For Study 2 data, the probability of testing positive was calculated from 
the logit using the formula: $\operatorname{prob}=\exp (\operatorname{logit}) /(1+\exp (\operatorname{logit}))$. The cut-off value was also converted into a probability using the same formula. Scatter plots of the probability of testing positive by time since the start of the trial and least squares means of these probabilities were created separately for the control and vaccinated groups in Study 2. In order to explore whether the logit was associated with time since infection, a generalized estimating equations model (GEE; to account for repeated measures within a badger) with a vaccination-time interaction term was conducted. The model used a Gaussian distribution with identity link and exchangeable correlation structure. Quadratic and logarithmic transformations of the independent variable "time since challenge" were carried out but did not yield lower values of QIC (quasilikelihood under the independence model criterion). The working correlation for the repeated effect was 0.288 . A two-way graph was created using the predictions of the GEE model and time since infection by vaccination status. All statistics were carried out using Stata version 12 (Stata Corp, College Station, TX, USA).

\section{Results}

\section{Test optimisation}

In total, 78 of the 215 samples $(36.3 \%)$ were infected with $M$. bovis based on culture results. Descriptive statistics for converted RLU response to each of the 8 antigens by infection status are presented in Table 1.

When using stepwise logistic regression with analytic weights, 7 of the 8 antigens were retained in the final model, but MPB70 peptide was not. The HosmerLemeshow test showed that the model fitted the data sufficiently well (p-value 0.29). Figure 1 depicts the ROC curve of the logit obtained for the combination of antigens. For a specificity of $99.99 \%$, a logit cut-off of -2.67 was needed. At this cut-off, the sensitivity was $30.77 \%$. Of the single antigens, MPB83 achieved the largest sensitivity, $24.36 \%$, with the specificity set at $99.99 \%$ (Figure 1).

\section{$\underline{\text { 2. Evaluation of test characteristics in vaccinated and non vaccinated badgers }}$}

In Study 1, 258 samples were analysed, with $30 \%$ of the badgers being tested 11 times and $70 \%$ of them 12 times. In Study 2, 297 samples were analysed (two samples could not be analysed for Rv3616c (fragment) due to insufficient serum, and were removed from the study), with a mean of 15.6 samples per badger ( $\mathrm{min}=9$, $\max =17$ ). A table showing the descriptive statistics for each of the 8 antigens as converted RLU, by infection and vaccination status, is presented as a supplementary file (Supplementary Table S2). This file also presents data for Study 1 samples originating from badgers vaccinated with the Danish strain. 


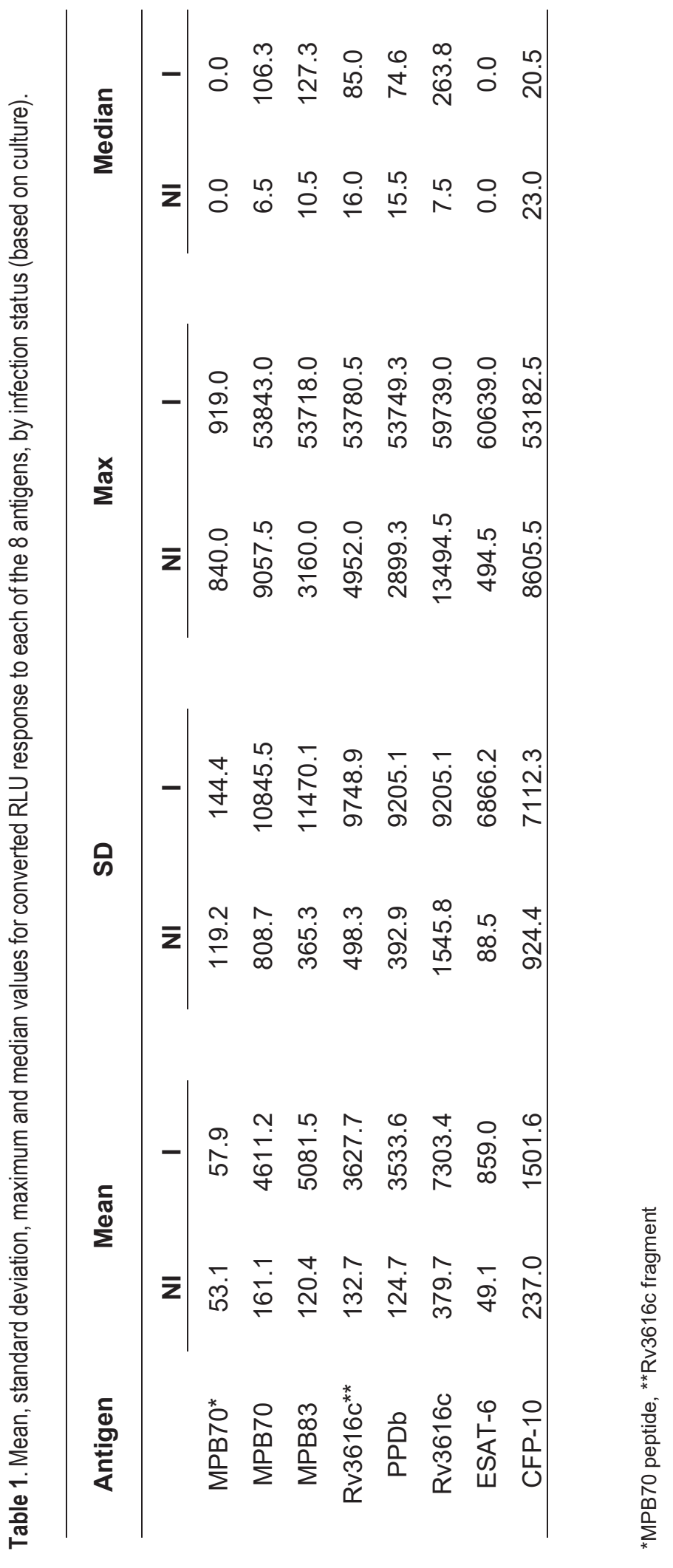




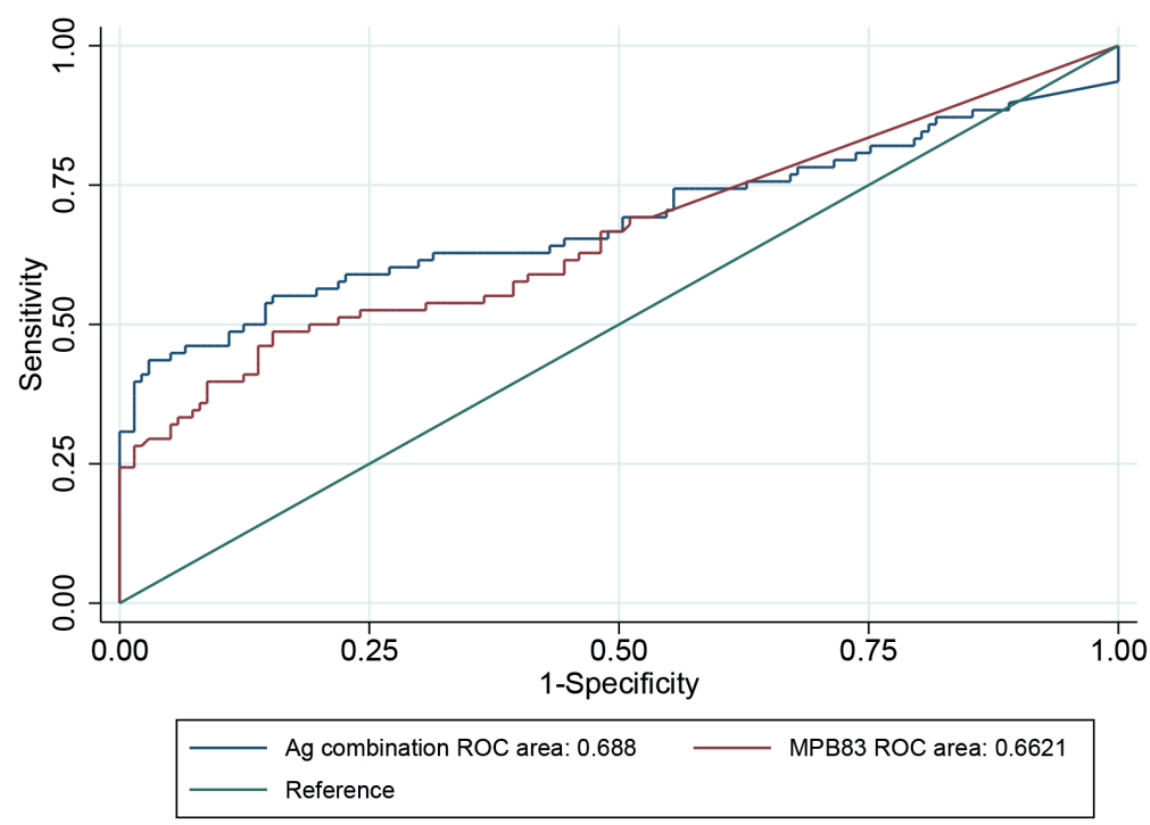

Figure 1. ROC curves of the logit obtained using either the optimised combination of antigens (blue line) or MPB83 (red line). Each is based on converted RLU values, and the green line is included for reference.

Using the optimal antigen combination, the mean sensitivity and specificity of the multiplex immunoassay test were respectively $22.99 \%$ (CI:14.64-33.25\%) and 78.95\% (CI:72.07-84.80\%) for Study 1, and 33.51\% (CI:26.76-40.81\%) and 83.04\% (CI: 74.78-89.47\%) for Study 2. The sensitivity and specificity were also calculated separately for vaccinated and non-vaccinated badgers from Study 2, obtaining sensitivity values for vaccinated badgers of $32.26 \%$ (CI:22.93-42.75\%) and specificity of $88.89 \%$ (CI:70.84-97.65\%); the equivalent values for non-vaccinated badgers were $34.78 \%$ (CI:25.15-45.43\%) sensitivity and 81.18\% (CI:71.24-88.84\%) specificity. 


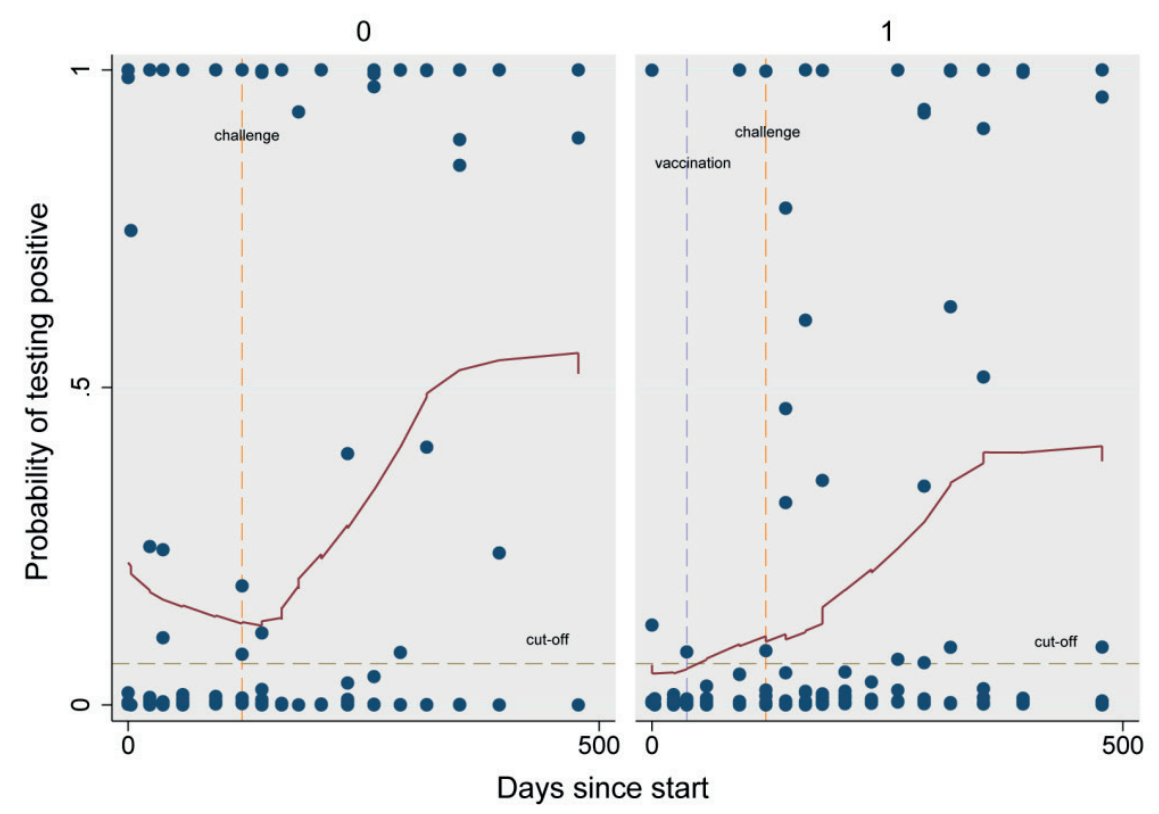

Figure 2. Scatter plot and least square means of the probability of testing positive for the control (left graph) and vaccinated (right) groups in Study 2 by time since the start of the study. Vertical reference lines showing the day of vaccination and challenge, and a horizontal reference line of the cut-off are included.

Figure 2 presents scatter plots and least square means of the probability of testing positive for the control and vaccinated groups in Study 2 by time since the start of the study.

A two-way graph showing the GEE predictions by time since challenge is presented in Figure 3. In this Figure, the rate of increase of the logit is presented separately for vaccinated and non-vaccinated badgers. The GEE analysis showed a significant association of the dependent variable with all three independent variables: time since challenge, vaccination and their interaction term $(\mathrm{p}<0.005)$. When a robust GEE model was fitted, the interaction term was borderline significant $(\mathrm{p}=0.045)$. In the Figure, a line showing the selected cut off value is highlighted. A reference line showing the minimum number of days to test positive for infected non-vaccinated badgers is also presented. 


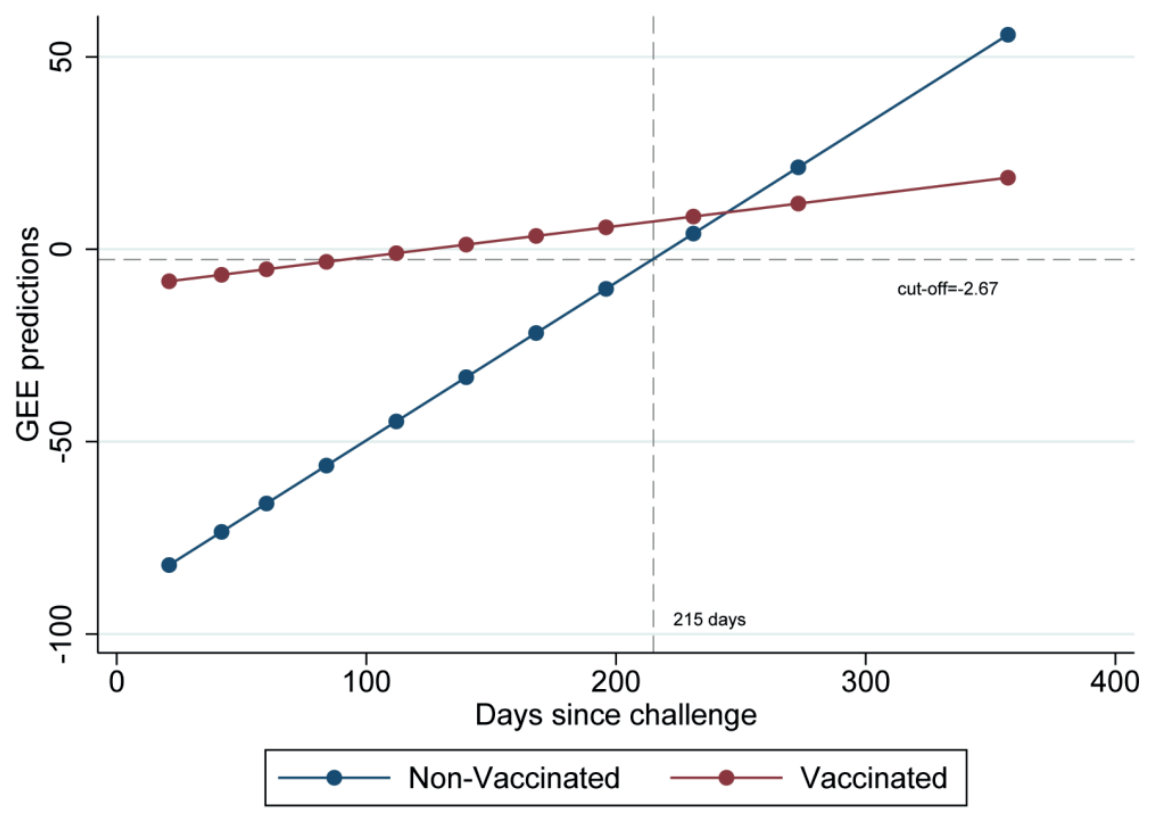

Figure 3. GEE predictions by time since challenge for vaccinated and non-vaccinated badgers. Two reference lines are presented: a vertical line showing the minimum number of days to test positive for infected non-vaccinated badgers and a horizontal cut-off line.

\section{Discussion}

The main objective of this paper was to optimise a diagnostic test for M. bovis infection in live badgers, for use during the evaluation of vaccine efficacy in a large bTB vaccine field trial in Ireland. On most occasions when a test is being developed, it is desirable to optimise both the sensitivity and the specificity of the test; in such situations, a cost ratio for false positives and false negatives of $1: 1$ is selected. However, in a previous study focusing on the statistical power of this trial [11], it was demonstrated that the diagnostic test needed to be tailored to achieve a specificity close to $100 \%$ to achieve a reasonable statistical power (60-80\%). As the incidence of $M$. bovis infection in the badger population decreases, there will be an increasing number of false positive results, randomly occurring in the mainly negative samples from both vaccinated and unvaccinated animals, with the potential to greatly bias estimates of vaccine efficacy. Hence, the need for specificity close to $100 \%$, thereby minimising the number of false positive results. Greiner et al. (2000) [27] have shown that analytic weights can be used to optimise cut-off values with regard to a specific cost ratio of false positive and false negative results. In order to reduce the number of false positive results, the upper left corner of the ROC curve (draw as sensitivity against 1-specificity) was optimised by selecting a cost ratio of 
false positives to false negatives equal to 100:1. Other ratios were explored, noting that cost ratios higher than 100:1 did not improve the ROC curve, probably due to the relatively small number of samples available to test. Subsequently, a cut-off value of a logit equal to -2.67 was needed to achieve a specificity of $99.99 \%$, resulting in a maximum sensitivity of $30.77 \%$. This sensitivity, although quite low in comparison to generally available diagnostic tests, is sufficient to achieve a statistical power of the vaccine trial of over $80 \%$ [11]. In this trial, test sensitivity is of lesser importance on study power because, when the incidence of M. bovis infection in badgers is low, the expected proportion of false negatives will be a fraction of something that is already a very small proportion.

In this study, culture was used to ascertain the disease status of individual badgers. Although it is unlikely that culture is a perfect 'gold standard', the methodology used in this study is based on an enhanced post mortem technique, currently the most sensitive available. In recent years, a progressive increase in estimated prevalence of $M$. bovis infection in badgers has been observed, both in Ireland and the UK, attributable to improved sensitivity of detection [3,4]. Crawshaw et al. (2008) [28] reported a $54 \%$ sensitivity of a standard post-mortem procedure compared to a more detailed enhanced post mortem technique.

A second objective in this paper was to assess the effects of vaccination on test characteristics. The optimised test described above was applied to data from Study 1 and 2. Badgers in Study 2 were followed for a much longer period of time subsequent to challenge than badgers in Study 1. Therefore data from Study 2, as opposed to Study 1, were used for further analysis. The sensitivities /specificities for non-vaccinated and vaccinated badgers in Study 2 were 34.78/77.97\% and $32.26 / 88.68 \%$, respectively. The test characteristics for both vaccinated and nonvaccinated badgers were very similar, as indicated by the overlap of the confidence intervals. Nonetheless, the specificity obtained for Study 2 was lower than that obtained when the test was optimised in naturally infected individuals (99.99\%). When looking at the specific badger data presented in Figure 2, it was observed that a large proportion of the false positives samples within the control group badgers belonged to two badgers that repeatedly tested positive prior to challenge (6 and 4 times per badger respectively, out of a total of 6 sampling times during this period). One possibility is that those two badgers were infected prior to the start of the experiment. However, blood samples from these animals were repeatedly screened before challenge by the more sensitive lymphocyte transformation assay using bovine and avian tuberculins, and were negative on all occasions (data not shown). The underlying cause of the false positive reactions in the two badgers is unknown though cross reactivity can potentially arise from concurrent infections with related pathogens or any microbe with shared epitopes, all of which potentially influence the specificity of this test. By removing samples from those two badgers, the 
specificity of the test increased to $93.62 \%$ in non-vaccinated badgers and to $91.00 \%$ for all badgers (vaccinated and non-vaccinated combined). Contrary to the control group, the false positives samples observed in the vaccinated group belonged to different badgers. These badgers tested positive (prior to challenge) on no more than one occasion, out of an average of 6 tests per badger during this period.

One of the concerns of serologically-based assays is that they detect infection later than assays based on the cell-mediated immune response, such as gamma-interferon. This is because the initial immune response is cell-mediated. A lag between infection and positive test results has been observed in the data obtained from Study 2 (Figures 2 and 3); this lag varies between vaccinated and non-vaccinated animals. From a biological point of view, we would expect that non-vaccinated badgers will carry a larger antigen load and thus will mount a larger antibody response to M. bovis challenge than those that are vaccinated. Nonetheless, it is the vaccinated group that shows the earliest positive test when a cut-off $=-2.67$ is selected (Figure 3 shows samples of vaccinated badgers testing positive earlier than 100 days after challenge, while the equivalent for the non-vaccinated group was 215 days). We can think of two possible interpretations for the observed results, one is that vaccinated badgers will mount a serological response faster than non-vaccinated badgers following infection, the other option is that observed results are due to chance (due to the small number of badgers in each group). It is possible that some of the badgers that were randomly allocated to the vaccinated group were extremely susceptible to infection and for those badgers, vaccination did not work.

Considering all of the above, what we propose in this study is that the multiplex immunoassay can be used to analyse the vaccine trial data, incorporating the optimal antigen combination identified from section 2.1 and a consistent cut-off of -2.67 . To account for the differences observed in the lag between time of infection and a positive test, we recommend that only subsequent captures that occur more than " $Y$ " days apart are used for the analysis, with "Y" being the minimum number of days necessary between infection and a subsequent positive test (215 days in this study). The number "Y" can be determined, after the vaccine trial dataset is gathered, as a trade-off between the increase in sensitivity and the possible reduction in power resulting from a decrease of our sample size. By taking this approach, it will be possible to minimise bias, specifically the incorrect classification of infected animals as non-infected.

In summary, a multi-antigen test has been optimised for use during the evaluation of vaccine effectiveness in a badger bTB vaccine field trial in Ireland. During optimisation, test sensitivity was estimated, while specificity was set at $99.99 \%$. Based on the operating characteristics of the diagnostic test, it has been demonstrated that the statistical power of the field trial could exceed $80 \%$ [11]. We have also 
observed that test characteristics do not vary greatly between vaccinated and nonvaccinated badgers. In relation to the time lag between infection and a positive test in vaccinated and non-vaccinated badgers, we have presented a strategy to enable the test to be used, and applied consistently, during trial evaluation.

\section{References}

1. Griffin JM, Williams DH, Kelly GE, Clegg TA, O'Boyle I, et al. (2005) The impact of badger removal on the control of tuberculosis in cattle herds in Ireland. Prev Vet Med 67: 237-266.

2. More SJ (2009) What is needed to eradicate bovine tuberculosis successfully: an Irish perspective. Vet J 180: 275-278.

3. Murphy D, Gormley E, Costello E, O'Meara D, Corner LAL (2010) The prevalence and distribution of Mycobacterium bovis infection in European badgers (Meles meles) as determined by enhanced post mortem examination and bacteriological culture. Res Vet Sci 88: 1-5.

4. Corner LAL, O'Meara D, Costello E, Lesellier S, Gormley E (2012) The distribution of Mycobacterium bovis infection in naturally infected badgers. Vet J 194: 166-172.

5. Murphy D, Gormley E, Collins DM, McGrath GE, Sovsic E, et al. (2011) Tuberculosis in cattle herds are sentinels for Mycobacterium bovis infection in European badgers (Meles meles): The Irish Greenfield Study. Vet Microbiol 151: 120-125.

6. Corner LAL, Costello E, Lesellier S, O'Meara D, Sleeman DP, et al. (2007) Experimental tuberculosis in the European badger (Meles meles) after endobronchial inoculation of Mycobacterium bovis: I. Pathology and bacteriology. Res Vet Sci 83: 53-62.

7. Corner LA, Costello E, Lesellier S, O'Meara D, Gormley E (2008a) Experimental tuberculosis in the European badger (Meles meles) after endobronchial inoculation with Mycobacterium bovis: II. Progression of infection. Res Vet Sci 85: 481-490.

8. Corner LAL, Costello E, Lesellier S, O'Meara D, Gormley E (2008b) Vaccination of European badgers (Meles meles) with BCG by the subcutaneous and mucosal routes induces protective immunity against endobronchial challenge with Mycobacterium bovis. Tuberculosis 88: 601-609.

9. Corner LAL, Costello E, O'Meara D, Lesellier S, Aldwell FE, et al. (2010) Oral vaccination of badgers (Meles meles) with $\mathrm{BCG}$ and protective immunity against endobronchial challenge with Mycobacterium bovis. Vaccine 28: 6265-6272.

10.Aznar I, McGrath G, Murphy D, Corner LAL, Gormley E, et al. (2011) Trial design to estimate the effect of vaccination on tuberculosis incidence in badgers. Vet Microbiol 151: 104-111. 
11.Aznar I, More SJ, Frankena K, De Jong, MCM (2013) Estimating the power of a Mycobacterium bovis vaccine trial in Irish badgers. Prev Vet Med 111: 297-303.

12.Greenwald R, Esfandiari J, Lesellier S, Houghton R, Pollock J, et al. (2003) Improved serodetection of Mycobacterium bovis infection in badgers (Meles meles) using multiantigen test formats. Diagn Micr Infec Dis 46: 197-203.

13.Dalley DJ, Hogarth PJ, Hughes S, Hewinson RG, Chambers MA (2004) Cloning and sequencing of badger (Meles meles) interferon [gamma] and its detection in badger lymphocytes. Vet Immunol Immunopathol 101: 19-30.

14.Chambers MA, Crawshaw T, Waterhouse S, Delahay R, Hewinson RG, et al. (2008) Validation of the BrockTB STAT-PAK assay for detection of tuberculosis in Eurasian badgers (Meles meles) and influence of disease severity on diagnostic accuracy. J Clin Microbiol 46:1498-1500.

15.Dalley D, Davé D, Lesellier S, Palmer S, Crawshaw T, et al. (2008) Development and evaluation of a gamma-interferon assay for tuberculosis in badgers (Meles meles). Tuberculosis 88: 235-243.

16.Chambers MA, Waterhouse S, Lyashchenko K, Delahay R, Sayers R, et al. (2009) Performance of TB immunodiagnostic tests in Eurasian badgers (Meles meles) of different ages and the influence of duration of infection on serological sensitivity. BMC Vet Res 5:42-42.

17.Drewe AJ, Tomlinson AJ, Walker NJ, Delahay RJ (2010) Diagnostic accuracy and optimal use of three tests for tuberculosis in live badgers. PLoS ONE 5(6): e11196.

18. Vordermeier HM, Chambers MA, Cockle PJ, Whelan AO, Simmons J, et al. (2002) Correlation of ESAT-6-specific gamma-interferon production with pathology in cattle following Mycobacterium bovis BCG vaccination against experimental bovine tuberculosis. Infect Immun 70: 3026-3032.

19.Gormley E, Doyle MB, McGill K, Costello E, Good M, et al. (2004) The effect of the tuberculin test and the consequences of a delay in blood culture on the sensitivity of a gamma-interferon assay for the detection of Mycobacterium bovis infection in cattle. Vet Immunol Immunopathol 102: 413-420.

20.Waters WR, Nonnecke BJ, Olsen SC, Palmer MV (2007) Effects of pre-culture holding time and temperature on interferon-gamma responses in whole blood cultures from Mycobacterium bovis-infected cattle. Vet Microbiol 119: 277-282.

21.Goodger J, Russell WP, Nolan A, Newell DG (1994) Production and characterization of a monoclonal badger anti-immunoglobulin $\mathrm{G}$ and its use in defining the specificity of Mycobacterium bovis infection in badgers by Western blot. Vet Immunol Immunopathol 40: 243-252.

22.Hewinson RG, Michell SL, Russell WP, McAdam RA, Jacobs WR (1996) Molecular characterization of MPT83: a seroreactive antigen of Mycobacterium tuberculosis with homology to MPT70. Scand J Immunol 43(5): 490-499. 
23.Greenwald R, Esfandiari J, Lesellier S, Houghton R, Pollock J, et al. (2003) Improved serodetection of Mycobacterium bovis infection in badgers (Meles meles) using multiantigen formats. Diagn Microbiol Infect Dis 46: 197-203.

24. Whelan C, Shuralev E, O'Keeffe G, Hyland P, Kwok, et al. (2008) Multiplex immunoassay for serological diagnosis of Mycobacterium bovis infection in cattle. Clin Vaccine Immunol 15: 1834-1838.

25.Whelan C, Whelan AO, Shuralev E, Kwok HF, Hewinson G, et al. (2010) Performance of the Enferplex TB assay with cattle in Great Britain and assessment of its suitability as a test to distinguish infected and vaccinated animals. Clin Vaccine Immunol 17(5): 813-817.

26. Whelan C, Shuralev E, Hyland P, Corner LAL, Murphy D, et al. (2009) Investigation of the immune response of Mycobacterium bovis infected European badgers (Meles Meles) using the Enfer Chemiluminescent Multiplex ELISA System. Poster session presented at: M. bovis V International Conference. August 25-28th; Wellington, New Zealand.

27.Greiner M, Pfeiffer D, Smith RD (2000) Principles and practical application of the receiver-operating characteristic analysis for diagnostic test. Prev Vet Med 45: 23-41.

28.Crawshaw TR, Griffiths IB, Clifton-Hadley RS (2008) Comparison of a standard and a detailed postmortem protocol for detecting Mycobacterium bovis in badgers. Vet Rec 163: 473-477.Murphy, D., Gormley, E., Costello, E., O'Meara, D., Corner L.A.L., 2010. The prevalence and distribution of Mycobacterium bovis infection in European badgers (Meles meles) as determined by enhanced post mortem examination and bacteriological culture. Res. Vet. Sci. 88, 1-5. 
Optimising and evaluating the characteristics of a multiple antigen ELISA for detection of Mycobacterium bovis infection in a badger vaccine field trial Supplementary material 


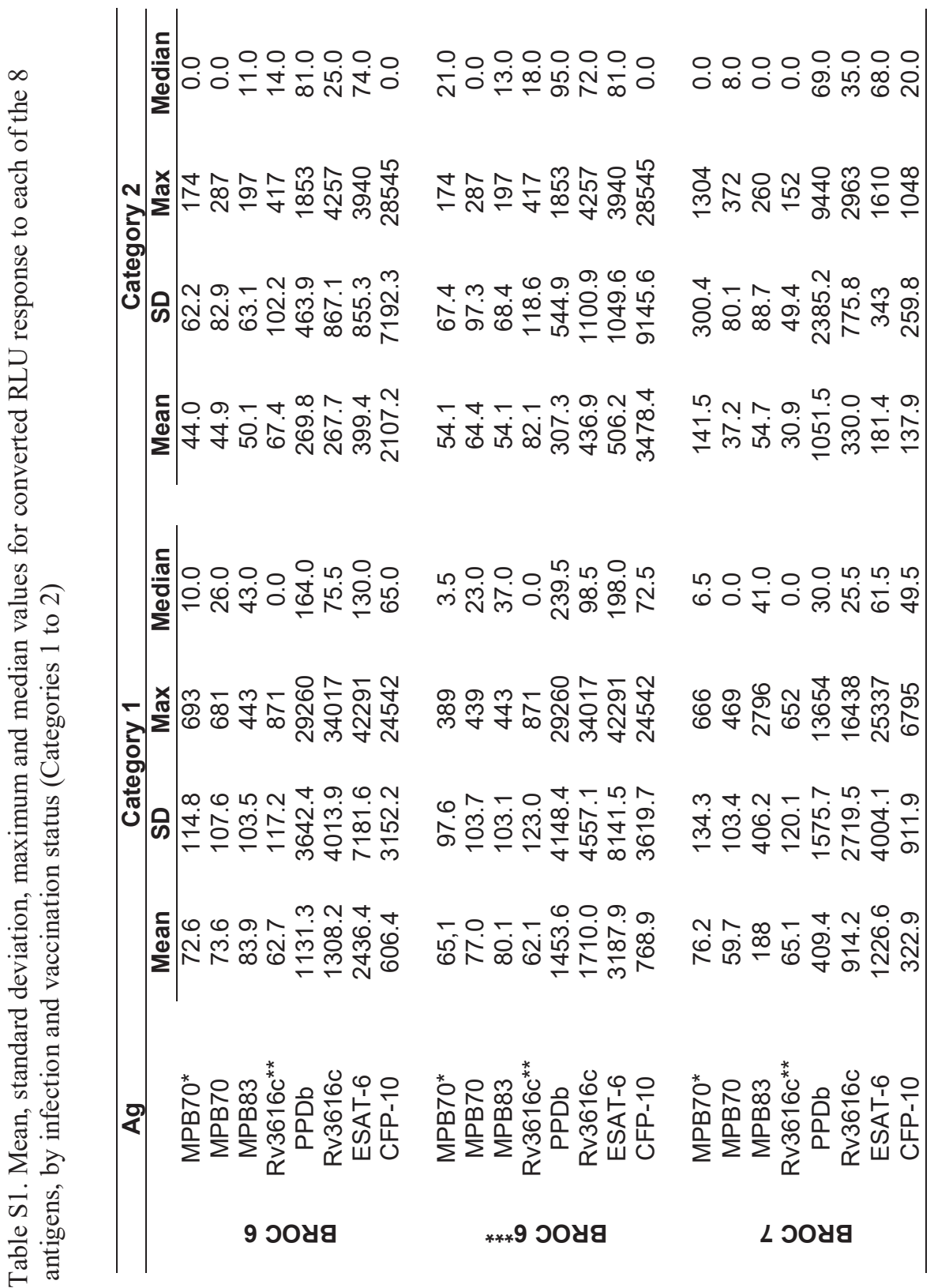




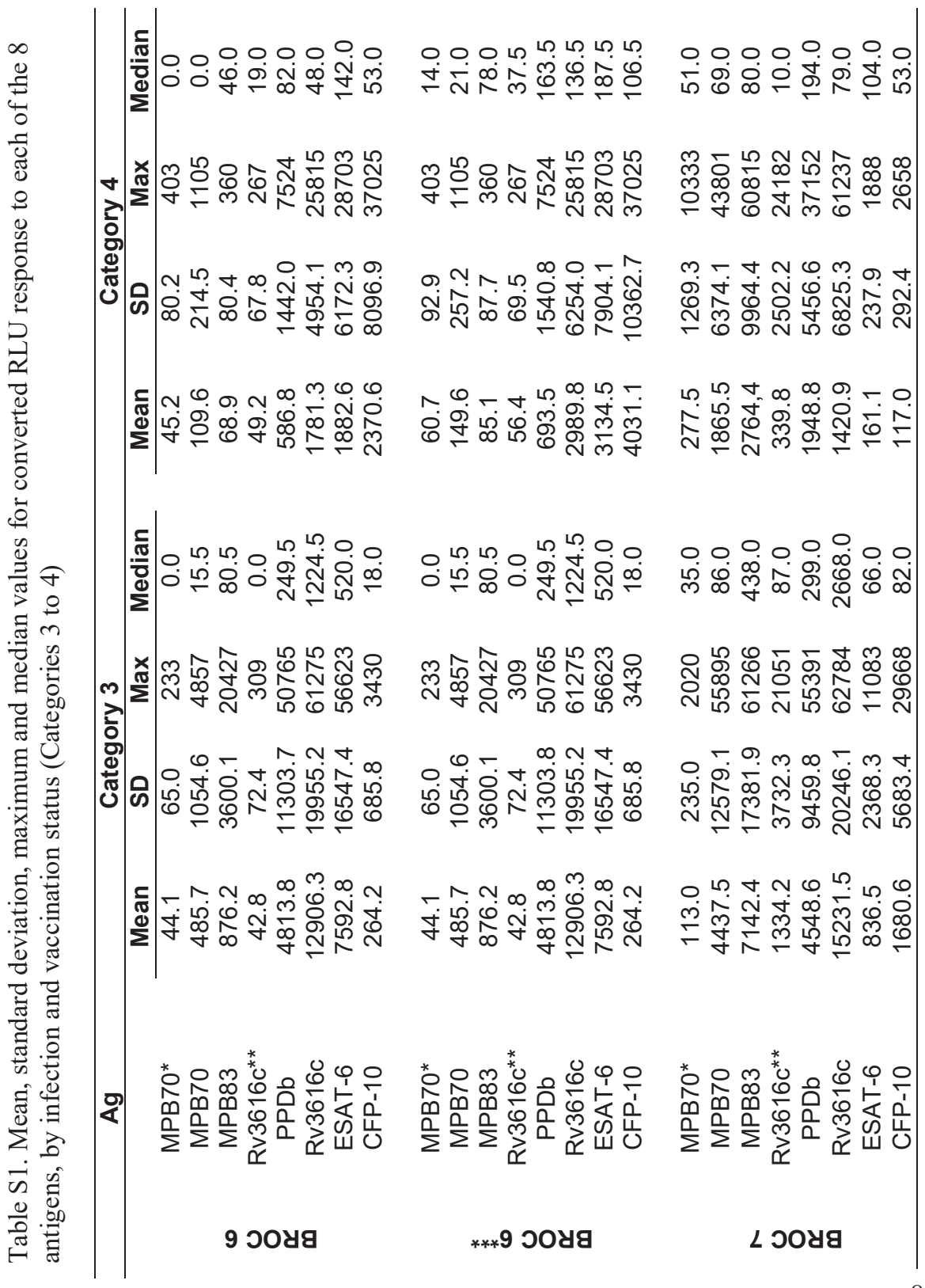



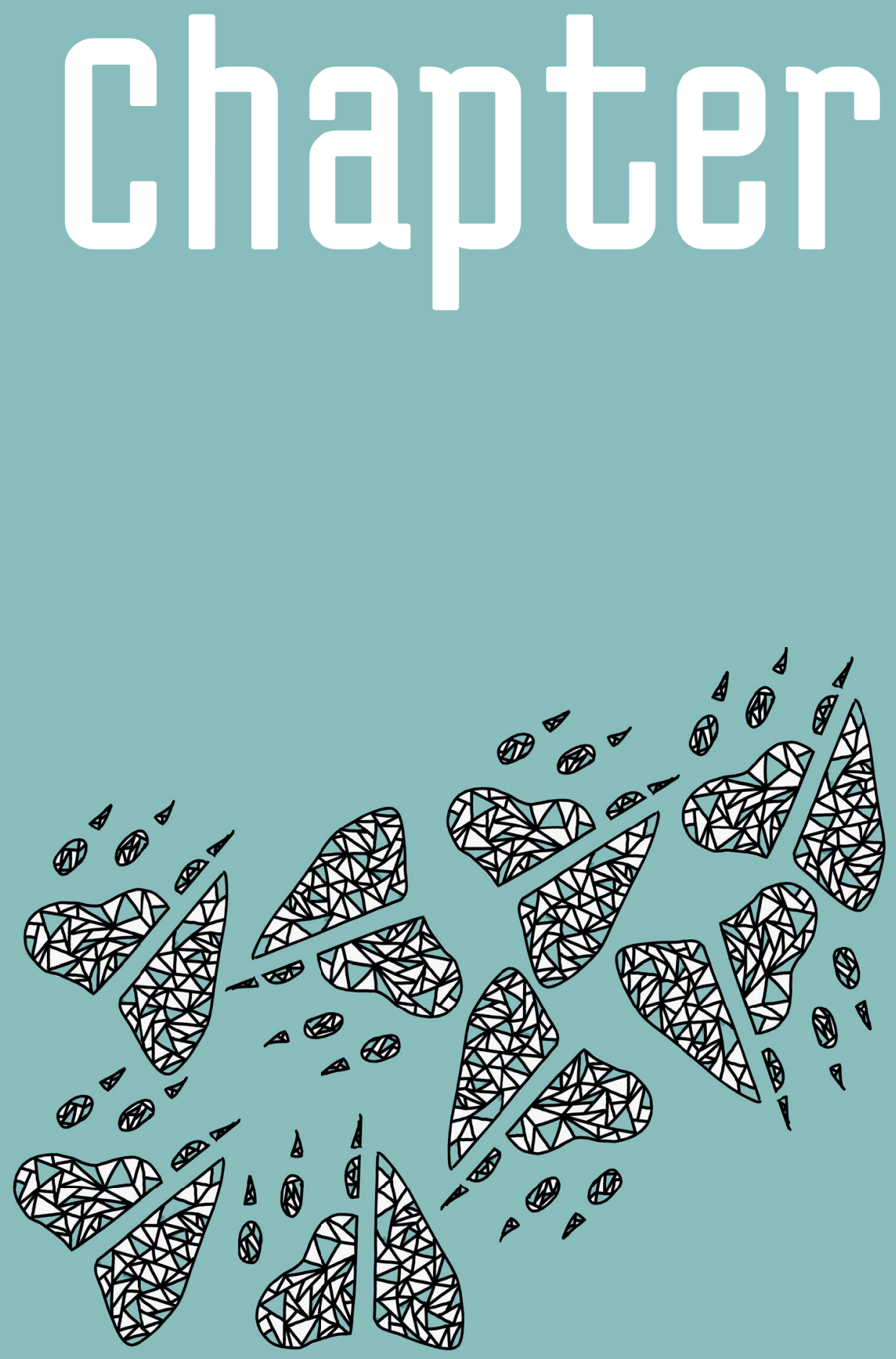


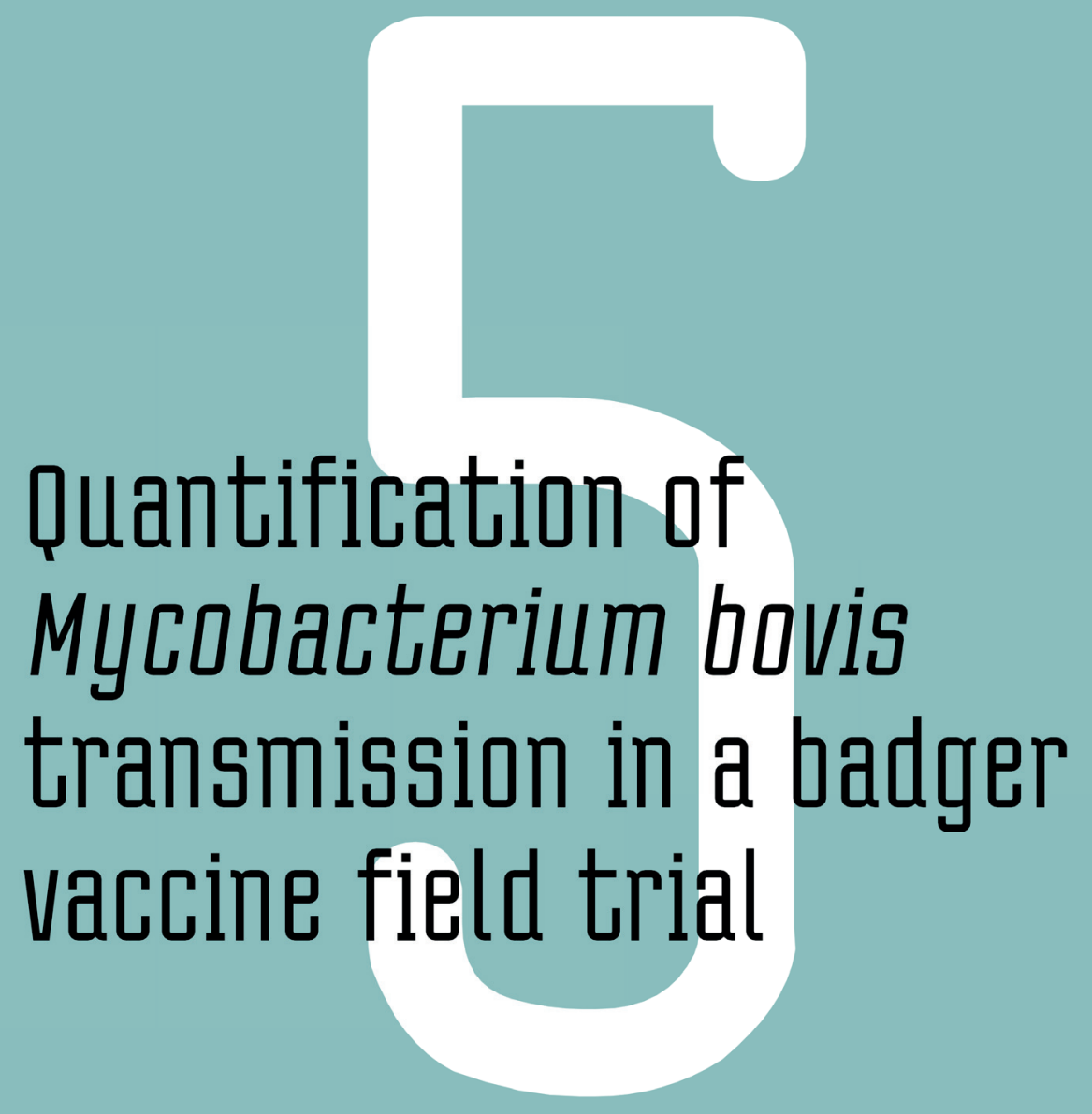

Aznar, I. , Frankena, H. , More, 5.J. , o'keeffe, J. , McGrath, G. , de Jong, M.C.M. 
Chapter 5

\title{
Quantification of Mycobacterium bovis transmission in a badger vaccine field trial
}

\author{
Aznar, I., Frankena, K., More, S.J., O’Keeffe, J., McGrath, G., de Jong, \\ M.C.M.
}

Preventive Veterinary Medicine (2018) 149, 29-37

(C) 2017 The Authors. Published by Elsevier B.V. This is an open access article under the CC BY-NCND license (http://creativecommons.org/licenses/BY-NC-ND/4.0/). 


\begin{abstract}
In the UK and Ireland, Bacille Calmette-Guérin (BCG) vaccination of badgers has been suggested as one of a number of strategies to control or even eradicate Mycobacterium bovis infection in badgers. In this manuscript, we present the results of a badger field trial conducted in Ireland and discuss how the novel trial design and analytical methods allowed the effects of vaccination on protection against infection and, more importantly, on transmission to be estimated. The trial area was divided into three zones North to South (A, B and C) where vaccination coverages of 0,50 and $100 \%$, respectively, were applied. Badgers were trapped over a 4 year period. Badgers were assigned to either placebo or vaccine treatment, with treatment allocation occurring randomly in zone B. Blood samples were collected at each capture, and serology was performed in these samples using a chemiluminescent multiplex ELISA system (Enfer test). The analysis aimed to compare new infections occurring in non-infected non-vaccinated badgers to those in non-infected vaccinated ones, while accounting for the zone in which the badger was trapped and the infection pressure to which this individual badger was exposed. In total, 440 records on subsequent trappings of individual non-infected badgers were available for analysis. Over the study period, 55 new infections occurred in non-vaccinated (out of $239=23.0 \%$ ) and 40 in vaccinated (out of $201=19.9 \%$ ) badgers. A Generalized Linear Model (GLM) with a cloglog link function was used for analysis. Statistical analysis showed that susceptibility to natural exposure with $M$. bovis was reduced in vaccinated compared to placebo treated badgers: vaccine efficacy for susceptibility, $\mathrm{VE}_{\mathrm{S}}$, was 59\% (95\% CI $\left.=6.5 \%-82 \%\right)$. However, a complete lack of effect from $\mathrm{BCG}$ vaccination on the infectivity of vaccinated badgers was observed, i.e. vaccine efficacy for infectiousness $\left(\mathrm{VE}_{\mathrm{I}}\right)$ was $0 \%$. Further, the basic reproduction ratio as a function of vaccination coverage (p) (i.e. R(p)) was estimated. Given that the prevalence of $M$. bovis infection in badgers in endemic areas in Ireland is approximately $18 \%$, we estimated the reproduction ratio in the unvaccinated population as $\mathrm{R}(0)=1.22$. Because $\mathrm{VE}_{\mathrm{S}}$ was now known, the reproduction ratio for a fully vaccinated population was estimated as $\mathrm{R}(1)=0.50$. These results imply that with vaccination coverage in badgers exceeding $30 \%$, eradication of $M$. bovis in badgers in Ireland is feasible, provided that the current control measures also remain in place.
\end{abstract}




\section{Introduction}

Bovine tuberculosis (bTB, caused by infection with Mycobacterium bovis) is a chronic inflammatory disease of bovidae (Bezos et al., 2014). A control/eradication programme for bTB in cattle started in Ireland in 1959 not only to address the economic losses associated with the infection (Caminiti et al., 2016), but also its zoonotic potential (Langer and LoBue, 2014). In the first ten years of the control programme, with a focus on measures to limit cattle to cattle transmission, the incidence of M. bovis infection in cattle was reduced from $17 \%$ to $0.5 \%$ (More and Good, 2006). Subsequently, progress has been slow, despite ongoing application of intense control strategies, which raised concerns about a role for one or more reservoirs of $M$. bovis maintaining transmission. Over the years, this hypothesis has been confirmed, including work highlighting high prevalence of infection in badgers (Meles meles) (Corner et al., 2005). Since then substantial research has been conducted to understand transmission of $M$. bovis between cattle and badgers, and of potential strategies capable of reducing this transmission. One such strategy is the use of BCG (Bacille Calmette-Guérin) badger vaccination (More and Good 2006). 


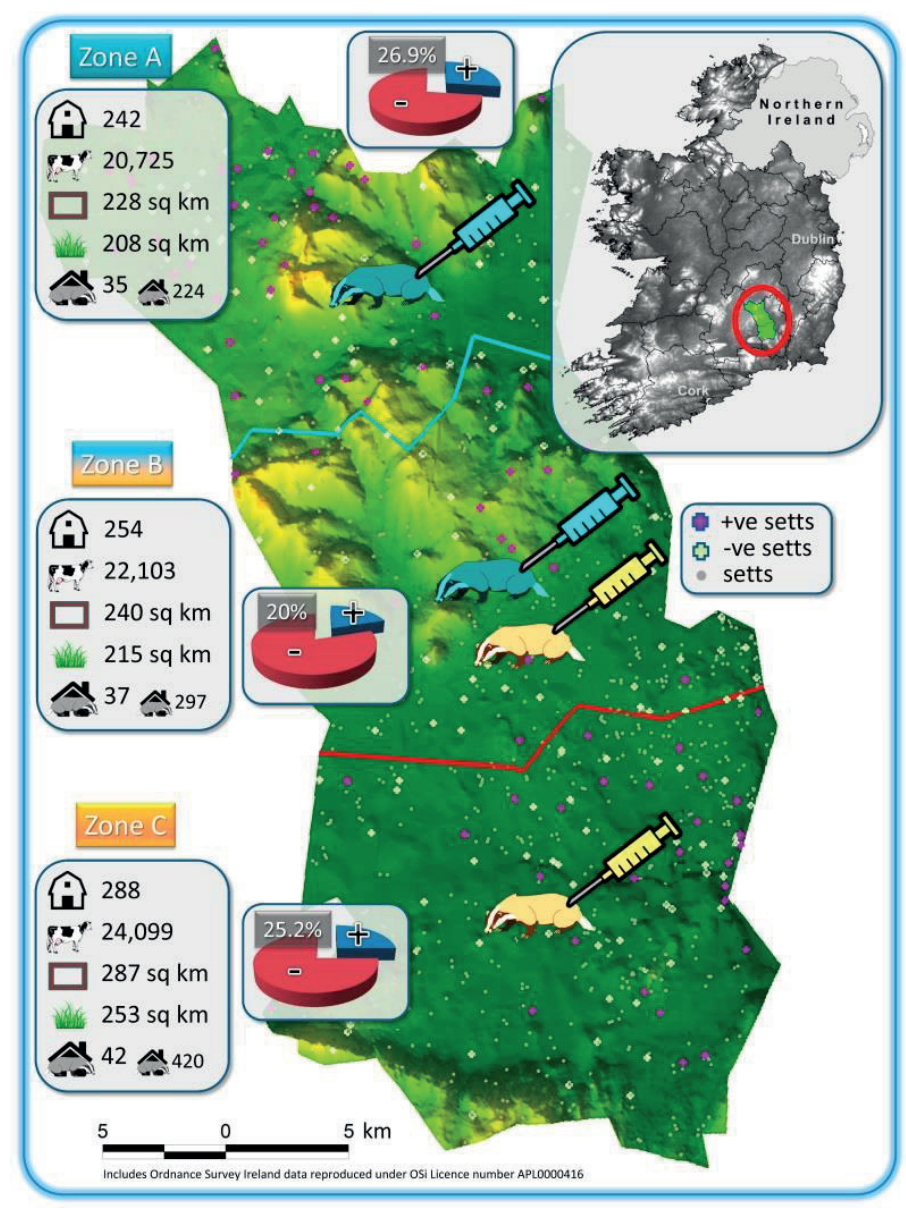

Figure 1. Topographic map of the Irish badger vaccine field trial showing: number of farms and bovines, zone area and area of farmed grassland (sq $\mathrm{km}$ ), and number of main and secondary badger setts per zone. From north to south, zones A, B and C indicate placebo (blue badger) and/or vaccine (yellow badger) allocation. Estimated M. bovis prevalence in badgers at the end of the first year is shown per zone (pie charts). Badger setts are represented as: all surveyed setts (grey dots), setts with at least one positive badger trapped in the first year (purple cross), setts with at least one negative badger trapped in the first year (light green cross). 
Experimental challenge trials with $M$. bovis following BCG vaccination by subcutaneous, mucosal, oral or intramuscular routes (Corner et al., 2008; Lesellier et al., 2009, 2011; Murphy et al., 2014) have demonstrated a reduction in disease progression in captive badgers. It has been proposed that this observed reduction in the number of sites with gross pathology and of general gross pathological severity scores observed in these badgers, could translate to a reduction of badger infectivity, and thus to a reduction in transmission in the field (Chambers et al., 2011). Here, the expected reduction in transmission due to a lower infectivity of badgers equates to what is known as vaccine efficacy for infectivity $\left(\mathrm{VE}_{\mathrm{I}}\right)$. In the human field, it is not uncommon to find vaccines that, by helping to reduce pathology and clinical symptoms in vaccinated and subsequently infected individuals, achieve a reduction of the infectivity of these individuals and, as a consequence, a reduction in transmission in the general population. Vaccines against smallpox, varicella, rubella, measles, hepatitis B and whooping cough have been recognized as having an important $V_{E}$ which contributes to the overall effect of these vaccines on the population (vaccinated and non-vaccinated), this overall effect being referred to as herd immunity (Fine et al., 1993; Halloran et al., 1999; Stephens et al., 2008).

In addition, protection of badgers against $M$. bovis infection could also be achieved as a consequence of reduced susceptibility. A reduction in susceptibility against infection would have both a direct and an indirect effect in the general population, i.e. vaccinated individuals are less likely to become infected (direct effect) and therefore, non-infected badgers are less likely to become infected if surrounded by these less susceptible individuals (indirect effect). Although this type of protection was not observed in laboratory trials, a reduction in susceptibility could potentially be attained under natural conditions because the infective dose that badgers are exposed to in the field is likely to be much lower than that used in experimental trials (Corner et al., 2008; Lesellier et al., 2011). This type of protection is referred to as vaccine efficacy for susceptibility $\left(\mathrm{VE}_{\mathrm{S}}\right)$. $\mathrm{VE}_{\mathrm{S}}$ solely refers to the direct effect.

Knowledge of both vaccine efficacies is important as overall transmission depends on both susceptibility and infectivity. However, methods to quantify transmission after vaccination have only been used in the last 20 years (Moerman et al., 1993; Stegeman et al., 1995; de Jong and Kimman, 1994). In 1994, de Jong and Kimman designed an experimental study that allowed quantification of the transmission observed in pigs vaccinated against pseudorabies virus. In subsequent experimental and field transmission studies, the effectiveness of vaccination was evaluated based on estimation of $\mathrm{R}(\mathrm{p})$ or the basic reproduction ratio as a function of the proportion of the population that is vaccinated (Moerman et al., 1993; Stegeman et al., 1995). $\mathrm{R}(\mathrm{p})$ is a crucial parameter to understand the impact of vaccination on population dynamics of $M$. bovis infection. If BCG vaccination is capable of reducing transmission between badgers, then estimates of the minimum vaccine coverage necessary to achieve eradication in badgers would be essential when designing an 
eradication program, based on Diekmann et al. (1990). By examining $R(p)$, the effects of combining vaccination with other control methods in the same or different species (e.g. the strategy of detection-and-removal of infected cattle from cattle herds) can be calculated. This is extremely important in the case of vaccination in badgers, as the ultimate goal is to help in the control or eradication of M. bovis infection in cattle.

Aznar et al. (2011) presented a novel design of a badger vaccination trial and developed a methodology to estimate both $\mathrm{VE}_{S}$ and $\mathrm{VE}_{\mathrm{I}}$ as well as $\mathrm{R}(\mathrm{p})$ based on incidence data (i.e. new M. bovis infections). The trial design consisted of three badger populations vaccinated with different vaccination coverages as suggested by Longini et al. (1998), but taking into account that these vaccination coverages are achieved over time rather than instantaneously. Here, we present the results of this badger vaccine/placebo field trial. M. bovis transmission among badgers was quantified as well as the effects of vaccination on the susceptibility and infectivity of badgers. Based on these results, the impact of badger vaccination on the M. bovis eradication program in Ireland is reviewed.

\section{Material and methods}

\subsection{Trial}

The badger vaccine field trial ran from 2009 until 2013. The trial area of approximately 750 square kilometres was divided into three zones north to south (A, $\mathrm{B}$ and $\mathrm{C}$ respectively) (see Figure 1). Using cages and stopped wire restraints, a capture-tag-release regime was established. Traps were fitted and left in the vicinity of every active sett for 10 days, with daily checks carried out by DAFM (Department of Agriculture, Food and the Marine) employees. After the 10 day period, traps were moved to different setts, taking approximately 23 weeks to cover the whole trial area (trappings occurring simultaneously in all three zones). Each 23 week period constituted a "sweep". A total of 8 sweeps were carried out over the length of the trial ( 2 sweeps per year). The last two sweeps involving some badger removal to allow for post-mortem evaluations (in the study of Gormley et al. (2017), sweeps 7 and 8 were combined and presented as sweep 7).

At first capture, each badger was tattooed and microchipped, with blood samples being collected at first capture and every subsequent recapture (Gormley et al., 2017). Vaccination with an oral BCG vaccine (Danish strain 1331, at a dose of $1 \mathrm{x}$ $10^{8} \mathrm{cfu}$ of BCG administered in the upper pharyngeal mucosa) suspended on a lipid formulation (Ancelet et al., 2012; Gormley et al., 2017) was applied randomly to $50 \%$ of the badgers trapped in zone B and to all badgers trapped in zone C. All badgers in zone A and the remaining $50 \%$ of the badgers trapped in zone $\mathrm{B}$ received a placebo. 
The Enfer chemiluminescent multiplex ELISA system (Whelan et al., 2008, 2010; Aznar et al., 2014) had been previously optimized to be used as the diagnostic test in this trial. The Enfer test optimization was conducted using data obtained from a population of 215 badgers trapped across 16 counties in Ireland (Murphy et al., 2010; Aznar et al., 2014). These badgers had been thoroughly examined and a large number of samples from tuberculous and non-tuberculous lesions were taken for culture (culture was used as the gold standard). Details about these badgers and culture methods are in Murphy et al. (2010). A study of factors affecting the statistical power of this design highlighted the importance of achieving close to $100 \%$ specificity in the diagnostic test used (Aznar et al., 2013). Therefore, the Enfer test was optimized to maximise sensitivity while retaining specificity at $99.99 \%$ in order to avoid loss of power that would arise from a number of false positive results randomly occurring in the mainly negative samples from both vaccinated and unvaccinated animals (Aznar et al., 2014). Test sensitivity did not play a major role in terms of study power, however, there was a need for consistent test performance among samples from all study animals throughout the trial period, including those vaccinated and not vaccinated. Many steps were taken to achieve this, including evaluating and comparing test results from vaccinated and non-vaccinated animals from experimental studies. Differences in terms of time to seroconversion were observed when the Enfer test was applied to vaccinated and non-vaccinated captive badger groups. As a result, a minimum time lag between two subsequent trappings of 215 days for all pairs of trappings was recommended (Aznar et al., 2014).

The trial was carried out under three licences issued by three different bodies: the Department of Health \& Children (research licence, B100/3187), the Department of Agriculture, Food \& the Marine (clinical trial licence, RL/08/06) and the Animal Research Ethics Committee of University College Dublin (ethics approval, ARECP-08-26).

\subsection{Datasets}

Two datasets were collected for analysis. The first consisted of data collected in the field (using handheld computers) by operators in charge of capturing and treating badgers in the trial area. This dataset contained information on 2,189 badger trappings (from the $1^{\text {st }}$ of September 2009 to the $12^{\text {th }}$ of July 2013). Data recorded on the handheld computers prior to the start of the trial and during the operator's training period were discarded (133 trapping records). Information recorded at each of the trappings were: badger identification (ID) data (badger ID, microchip and tattoo numbers), badger's sett ID, date of examination, presence of ectoparasites (ticks, fleas, lice) and injuries, demographic data (age, sex, weight), type of diagnostic samples taken (faecal swabs, blood samples, pharyngeal swabs, DNA 
samples, others), vaccination data (date of vaccination, vaccine code), operator name, comments, trial zone (A, B or C), and sweep number (1-8).

The second dataset, consisting of 1,800 records, contained diagnostic test information of blood samples taken each time a badger was trapped. Blood samples were tested using the Enfer multiple antigen ELISA system for detection of M. bovis antibodies (Enfer Scientific, Co. Kildare, Ireland). Antibody responses were expressed as relative light units (RLU) to a panel of 8 antigens: MPB83, MPB70, Rv3616c fragment and full protein, ESAT-6 and CFP10, as well as purified protein derivative from $M$. bovis (PPDb) and a peptide of MPB70. The optimization process is described in detail by Aznar et al. (2014). Blood samples were analysed twice with the Enfer test: first after the end of each sweep, and a second time after the vaccine trial had ended. When both sets of results were compared, low repeatability for two antigens (MPB70 and Rv3616c fragment) was observed. These two antigens were removed prior to the final test optimization. The optimization was carried out using the second set of test results and after removing the two mentioned antigens. For that, a stepwise logistic regression with analytical weights (to optimize specificity versus sensitivity) on the converted RLU obtained for the six remaining antigens was carried out (Aznar et al., 2014). By assessing the ROC curve for the model results, a cut off value equal to -1.95 was selected to achieve $99.99 \%$ (exact confidence interval: $97.34-100 \%$ ) specificity and $25.33 \%$ (exact confidence interval: 20.80 $42.24 \%$ ) sensitivity (exact intervals instead of confidence intervals were calculated as specificity was very close to $100 \%$ ). Blood samples were classified as positive or negative based on this cut off value.

\subsection{Data collation}

The two datasets, containing capture and serology data were merged (1,759 trapping records). Data were collated to be analysed as a Bernoulli experiment. For that purpose, the full dataset was organized so that each entry contained information regarding two subsequent trappings of a single badger (vaccinated or nonvaccinated) that tested negative at the initial trapping. The first entry for an individual badger was recorded the second time that a specific badger was trapped. A badger that tested negative at its second trapping could then initiate a new record in our dataset if trapped for a third time, and so forth. Each entry line contained information on: infection status of the badger at the initial and current trapping, current and previous examination date, sweep number and zone where the badger was trapped each time, whether the badger had been allocated to vaccine or placebo, and date of treatment. Once a badger was allocated to either vaccine or placebo, it remained as such for the rest of the study. Prior to the analysis, three variables were calculated from the data recorded in the handheld computers including: delta $t(\Delta \mathrm{t})$ (i.e. time in days between two subsequent trappings of an individual badger), and prevalence (Prev) and fraction of infected vaccinated badgers ( $F i$; the fraction of the 
total number of infected badgers that became infected after vaccination) at the beginning of $\Delta \mathrm{t}$ in the zone where the badger was trapped. A badger allocated to the vaccine treatment was considered vaccinated the day after receiving the vaccine. Therefore, as we knew whether the badger had been allocated to the vaccine or placebo treatment, a new variable Vaccine status $\left(V_{S}\right)$ was created that coded 0 for badgers allocated to the placebo treatment (also for badgers allocated to the vaccine treatment on the first date of treatment) and 1 for vaccinated badgers trapped at least one day after they received the first vaccination.

\subsection{Statistical analysis}

The data collation, as well as the descriptive and statistical analyses, were carried out using Stata ${ }^{\circledR}$ (version 14; Stata Corp., College Station, TX, USA). As part of the descriptive analysis, crude transmission rate parameters (beta transmission parameters) were calculated as the number of new cases divided by number of susceptibles and prevalence in each sweep, for the three zones. In order to help in visualizing patterns, a non-parametric regression of the beta transmission parameters (lowess smoothing) was conducted.

The purpose of the statistical analysis was to compare new infections occurring in vaccinated non-infected badgers to those occurring in non-vaccinated non-infected ones while taking account of both the infection pressure these badgers were exposed to and the trial zone (A, B or C) badgers had been trapped in (Aznar et al., 2011). Data on 440 pairs of trappings (subsequent trappings of individual badgers) were used in the statistical analysis. Only badgers that tested negative at the initial trapping were included. Badgers were coded either 1 or 0 , respectively, depending on whether or not they tested positive at the subsequent trapping.

Assuming "separable mixing", whereby transmission depends only on the infectivity of the donor and the susceptibility of the receptor (Diekmann et al., 1990), the expected infection status of any uninfected re-trapped badger (vaccinated or nonvaccinated), was modelled in the total population using a generalized linear model (GLM). With this model we aimed to explain new infections from three explanatory variables: a) the vaccination status of the badger, b) the fraction of infected vaccinated badgers, and c) the zone where the badger was trapped. Details of the statistical model are elaborated below. If vaccination is effective, then we would expect infectivity to vary both between the three zones and also over time due to differences in the fraction of infected badgers that were vaccinated. It is important to note that the percentage of vaccinated badgers increased over the duration of the trial in zones B and C (Figure 2).

The expected number of cases per unit of time $\mathrm{E}(C)$ can be formulated as $\mathrm{E}(C)=\mathrm{S} \cdot(1-$ $\left.\mathrm{e}^{-\beta \cdot \Delta \mathrm{t} \cdot \operatorname{Prev}_{\mathrm{t}}}\right)$ where $\mathrm{S}$ is the number of susceptible badgers and $\left(1-e^{-\beta * I * \frac{\Delta t}{N}}\right)$ is the 
probability that any of the susceptible badgers becomes infected (supplementary material, Section 1). Then the complementary log-log (cloglog) link function results in an estimate for $\log (\beta)$ taking $\ln \left(\right.$ Prev $^{*} \Delta t$ ) as offset (Aznar et al., 2011). This model was run separately for vaccinated and non-vaccinated badgers, allowing separation of the effects of vaccination in susceptibility and in infectivity as explained in derivations presented in Section 1 of the supplementary material. By separating these two effects, estimations of $\mathrm{VE}_{S}$ and $\mathrm{VE}_{\mathrm{I}}$ are possible. The model used was:

$$
\text { cloglog } E(C)=\beta_{0}+\beta_{1, B} Z_{B}+\beta_{1, C} Z_{C}+\beta_{2} V s+\beta_{3} F i+\text { offset }
$$

where $Z$ codes for zone (binary dummy variable $0 / 1$ for each of the zones, zone A being the reference), $V S$ is the vaccination status of the recipient badger, $F i$ is the fraction of vaccinated badgers among the infected badgers at the beginning of the time interval in that same zone, and $\beta_{0}, \beta_{1, B}, \beta_{1, C}, \beta_{2}, \beta_{3}$, are the regression coefficients as estimated by our model. For modelling purposes, once a badger tested positive to the serological test, it was considered positive for the rest of the study and therefore subsequent trappings of this badger were not included in the analysis. As the number of predictors in the maximum model was small, all possible combinations of predictors were examined (including interaction terms). The final model was selected based on the lowest value for the Akaike Information Criterion (AIC).

From this model, four transmission parameters: $\beta_{\mathrm{vv}}, \beta_{\mathrm{vu}}, \beta_{\mathrm{uv}}$ and $\beta_{\mathrm{uu}}$ were estimated. The first sub-index in these transmission parameters indicates the vaccination status of the badger transmitting M. bovis (whether it is from a vaccinated (v) or nonvaccinated badger $(u)$ ), while the second sub-index refers to the vaccination status of the recipient badger. The two vaccine efficacies and $R(p)$ can then be calculated from these four transmission parameters (see Section 1 of the supplementary material). Using the regression coefficients from our model, the transmission rate parameters, ignoring zone effects, can then be estimated as:

$\beta_{u v}=e^{\beta_{0}+\beta_{2}}, \beta_{v v}=e^{\beta_{0}+\beta_{2}+\beta_{3}}, \beta_{u u}=e^{\beta_{0}}$ and $\beta_{v u}=e^{\beta_{0}+\beta_{3}}$

Vaccine efficacies were calculated as:

$V E_{s}=1-\frac{\beta_{u v}}{\beta_{u u}}=1-\frac{\beta_{v v}}{\beta_{v u}}=1-e^{\beta_{2}}$ and $V E_{I}=1-\frac{\beta_{v u}}{\beta_{u u}}=1-\frac{\beta_{v v}}{\beta_{u v}}=1-e^{\beta_{3}}$,

noting that coefficient $\beta_{2}$ calculated for the variable $(V s)$ contributes to the estimation of $V E_{S}$, and the coefficient $\beta_{3}$ calculated for the variable $(F i)$ contributes to the estimation of $V E_{I}$, thus being able to estimate both vaccine efficacies. The reproduction ratio as a function of the proportion (p) of badgers vaccinated $R(p)$ was determined as:

$R(p)=(1-p) \cdot R(0)+p \cdot R(1)$

Where $\mathrm{R}(0)=\frac{1}{1 \text {-prevalence }}$ and $\mathrm{R}(1)=\left(1-V E_{S}\right) \cdot\left(1-V E_{I}\right) \cdot \mathrm{R}(0)$ 


\section{Results}

\subsection{Vaccine field trial descriptive analysis}

Overall, 1,093 badgers were trapped over the 8 sweeps, with 435 badgers trapped in zone A, and 243 and 415 in zones B and C, respectively. In total, 673 badgers were trapped once, 253 twice, 111 three times, 38 four times, 13 five times and 5 six times. An initial concern over the vaccine trial design was the fact that no major physical boundaries existed between the three zones. A large number of badger movements across the three zones could have hampered the vaccination gradient between the zones and therefore reduced the power of the analysis. Such a large movement was not expected, nonetheless we can confirm that it did not occur as only in $2 \%$ (22) of the subsequent trapping events had badgers originally trapped in one zone been trapped in a different zone at a later stage.

The prevalence of $M$. bovis infection, estimated as the overall percentage of positive trappings to the Enfer test at each sweep, ranged between $12.5 \%$ and $37.8 \%$ (see Table S1 in Section 2 of the supplementary material). At the beginning of the trial, the zone prevalence (the percentage of positive trappings in each zone in sweep 1) was higher, but not statistically different, in zone A (31.7\%) compared to zones B $(19.0 \%)$ and C $(23.5 \%)$ (p-value $=0.14)$. During the first year of the trial (that is, considering sweeps 1 and 2 together to avoid the effect of seasonality on trapping efforts), the prevalence was also highest in zone A (26.9\%) compared to zones B and C ( $20 \%$ and $25.2 \%$, respectively) but again, these differences were not statistically significant $(p$-value $=0.49)$. Due to a procedural error, blood results for 70 samples taken from badgers during sweep 2 were not available (see Section 4 of the supplementary material). The incidence of $M$. bovis infection per sweep, defined as the number of newly infected badgers (captured badgers that tested positive for the first time in sweep $n$ ) divided by the number of susceptible badgers (badgers trapped in sweep $n$ that had never tested positive or tested positive for the first time in that sweep), varied over time and across zones, with the lowest incidence being in sweep 5 in zone C (see Figure S1 in Section 2 of the supplementary material). In zones B and $\mathrm{C}$, the proportion of $\mathrm{BCG}$ vaccinated badgers increased from sweep 3 to 6 , then decreased in sweeps 7 and 8 (as the last sweeps involved badger removal) (Gormley et al., 2017). At sweep 6, the proportion of vaccinated badgers in zones $B$ and $C$ were $37.3 \%$ and $62.2 \%$ respectively (Figure 2 ). 

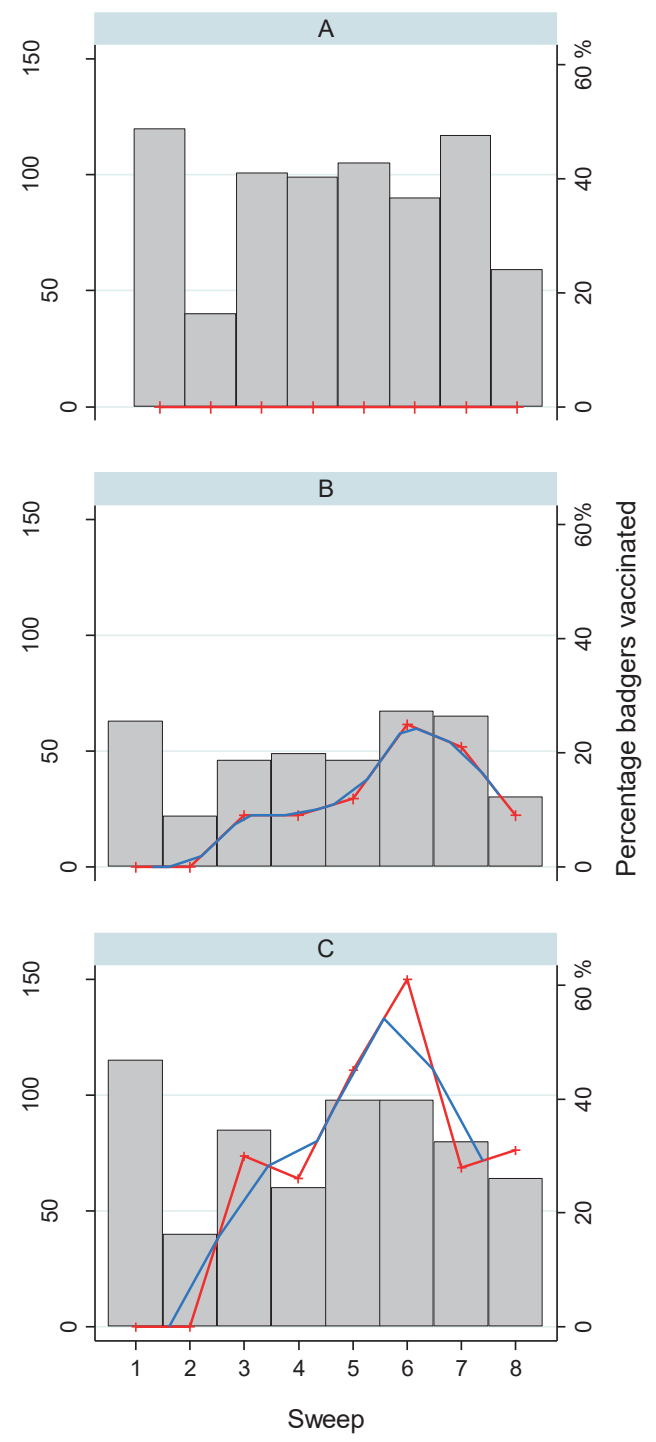

Figure 2. Total number of badgers caught at each sweep (left vertical axis) and percentage of captured badgers that were vaccinated (right vertical axis), including polynomial $(n=4)$ smoothing of this percentage per sweep and zone (right vertical axis). 
Crude transmission rate parameters in each sweep, for the three zones, and a lowess smoothing of the transmission parameters are presented in Figure 3. During the trial, there was a non-significant decrease in these crude transmission rate parameters in zones $\mathrm{B}$ and $\mathrm{C}$. The possible change over time in crude transmission rate was less clear for zone A. However, the overall initial transmission (at sweep 3) in this zone (i.e. even before vaccination could have had an impact) was already lower compared to the other two zones (Figure 3).

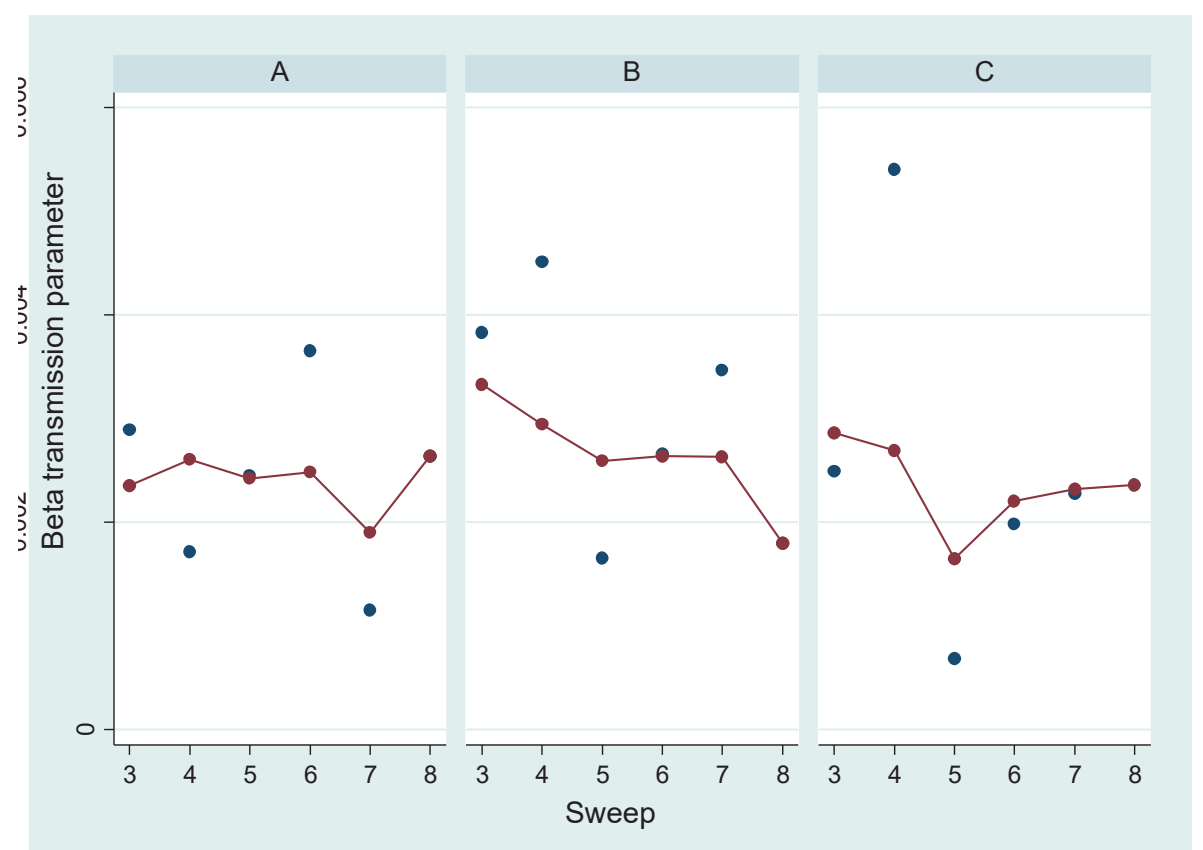

Figure 3. Crude transmission rate parameters (beta, in blue) and lowess smoothing (in red) per sweep estimated separately for the three trial zones.

\subsection{Statistical analysis}

The dataset consisted of 440 records (239 originated from non-vaccinated badgers and 201 from vaccinated badgers). A total of $55(23.0 \%)$ and $40(19.9 \%)$ new infections occurred in non-vaccinated and vaccinated badgers, respectively. Vaccination status of the badger receiving the vaccine was the only statistically significant explanatory variable in the model. Nonetheless, all variables (except the interaction terms) were kept in the final model as that was the model with the lowest AIC (490.2). Using the coefficient obtained for recipient vaccination status, we calculated vaccine efficacy for susceptibility, $\mathrm{VE}_{\mathrm{S}}$, as $59 \%(95 \% \mathrm{CI}=6.5-82 \%)$; that is, a $59 \%$ reduction in susceptibility of vaccinated compared to unvaccinated badgers 
was achieved (see Table 1), but no significant effect of vaccination on infectivity was observed in this trial (hence $\mathrm{VE}_{\mathrm{I}}=0 \%$ ).

Table 1. Results of the final generalized linear model including the estimated regression coefficient, $p$-value and 95\% confidence interval for all explanatory variables (fraction of infected vaccinated badgers, vaccination status and zone) and constant in a model fitted in data from all three zones of the Irish badger vaccine field trial. Only Vs, the vaccination status of the recipient, is significant, with the other variables retained to control for confounding.

\begin{tabular}{|c|c|c|c|}
\hline Variable & Coef & p-Value & $(95 \% \mathrm{Cl})$ \\
\hline Constant & -6.07 & $<0.001$ & -6.38 to -5.77 \\
\hline \multicolumn{4}{|l|}{ Zone } \\
\hline$A$ & & Refere & \\
\hline B & 0.55 & 0.083 & -0.07 to 1.17 \\
\hline C & 0.63 & 0.193 & -0.32 to 1.58 \\
\hline Vs & -0.90 & 0.034 & -1.73 to -0.07 \\
\hline $\mathrm{Fi}$ & 1.37 & 0.119 & -0.35 to 3.10 \\
\hline
\end{tabular}

In addition to the main model, two more statistical analyses were conducted. As Zone $\mathrm{B}$ resembles the classic 50:50 vaccine-placebo trial design (but with a change in vaccination coverage over time and the availability of longitudinal data on infection), estimation of the direct effect of vaccination on susceptibility was possible in this zone only. The model showed a similar outcome for $\mathrm{VE}_{\mathrm{S}}(54 \%, 95 \% \mathrm{CI}=0.0-79.9 \%)$ (see Table S2 in Section 3 of the supplementary material). A lower initial crude beta transmission parameter was observed in zone A compared to zones B and C (Figure 3), for reasons that are not clear. Due to this lower initial transmission parameter observed in zone A, and as our design only required a minimum of two populations vaccinated at different vaccination coverages, the model was run again using data from zones B and C only. Similar results were obtained in terms of both the effect of vaccination on susceptibility and infectivity, with $\mathrm{VE}_{\mathrm{S}}=60 \%(95 \% \mathrm{CI}=8.8$ $83.0 \%)$ and no significant effect of vaccination on infectivity $\left(\mathrm{VE}_{\mathrm{I}}=0 \%\right)$ (see Table $\mathrm{S} 3$ in Section 3 of the supplementary material).

We finalized our analyses by estimating $R(p)$ for a range of vaccine coverages, as it is the impact of the combination of both vaccine efficacies that determines the feasibility of using vaccination as a strategy to achieve $M$. bovis eradication in badgers. The average $M$. bovis prevalence in badgers in Ireland declined between 2007 to 2013, with an average prevalence from May 2007 to May 2011 equal to 17.7\%, and from June 2011 to April 2013 equal to 9.9\% (Byrne et al., 2015). For any population where an infection is at the endemic steady state, the fraction of 
susceptible individuals equals $1 / \mathrm{R}$. Thus, for a badger prevalence equal to $18 \%$, we can calculate $\mathrm{R}(0)=\frac{1}{1-\text { prevalence }}=1.22$, and for a prevalence equal to $10 \%$, $\mathrm{R}(0)=1.11$. As $\mathrm{VE}_{\mathrm{S}}=59 \%$ and using the higher prevalence estimate $(18 \%)$, the reproduction ratio for a fully vaccinated population can be calculated as $\mathrm{R}(1)=(1-$ $\left.V E_{S}\right) * \mathrm{R}(0)=0.50$. These results indicate that adding vaccination to the current control strategies in Ireland, eradication of $M$. bovis infection in badgers can be achieved with any vaccination coverage above 30\% (Figure 4).

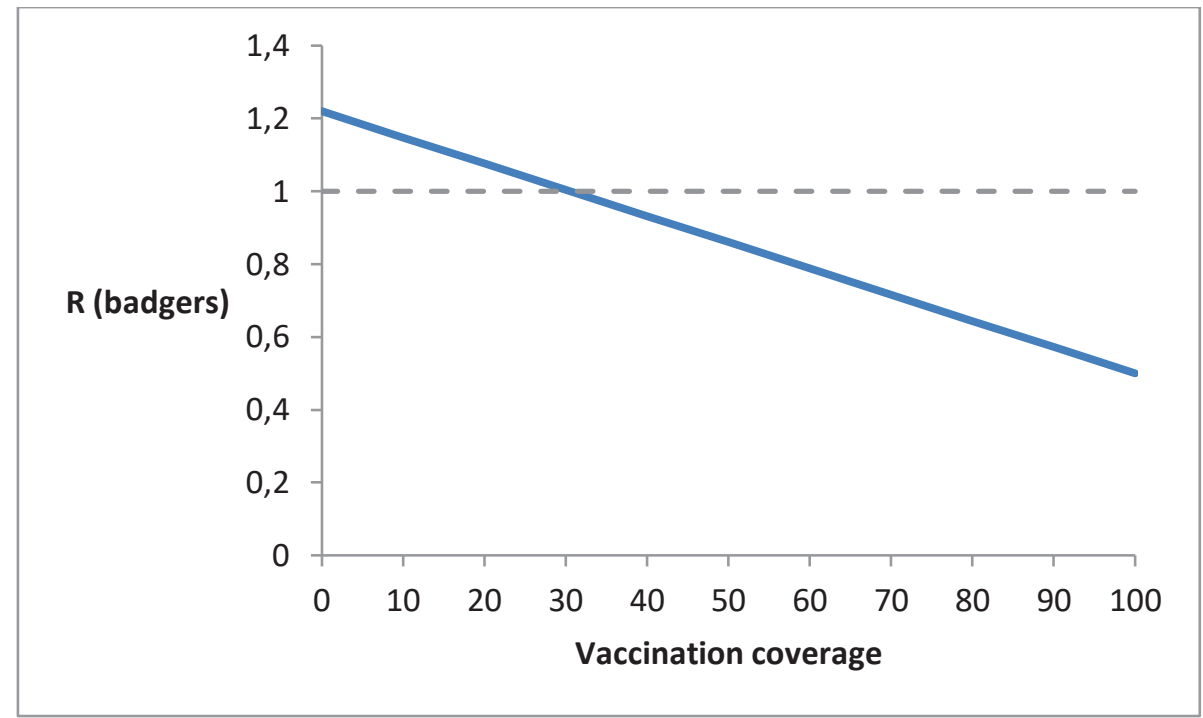

Figure 4. Basic reproduction ratio for badger to badger transmission as function of vaccination coverage, given $\mathrm{R}(0)=1.22$ and a $\mathrm{VE}_{\mathrm{S}}=59 \%$.

\section{Discussion}

In this manuscript, the effect of BCG vaccination on M. bovis transmission between badgers in the field has been quantified for the first time. Here, separate estimates on the effects of vaccination on both protection against infection and on the infectivity of badgers that become infected subsequent to vaccination are presented. The vaccine efficacy estimates presented in this paper contribute to a better understanding of the biological processes underpinning the protection against transmission achieved by BCG vaccination in the field. While no direct protection against infection following vaccination was reported in experimental trials (where vaccinated badgers were challenged with different doses and different strains of $M$. bovis) (Corner et al., 2008, 2010), we observed a 59\% protection against infection of vaccinated badgers in the field. The difference between our findings and those obtained in laboratory trials is not surprising, as the route of infection, infection dose, 
number of infection events to achieve this dose, etc occurring in the wild are unknown. Nonetheless, we cannot confirm whether or not the observed protection against infection is due to a lower infection dose in the wild compared to experimental trials (Corner et al., 2008, Lesellier et al., 2011). A reduction in the total infectivity of vaccinated and subsequently infected badgers in the field had been anticipated based on the reduction in disease progression observed in vaccinated compared to non-vaccinated badgers in experimental studies (Chambers et al., 2011). However, no reduction of infectivity was found in our study. The lack of effect of $B C G$ vaccination on infectivity in the general badger population is thus at odds with the hypothesis that vaccination, by reducing disease progression, reduces the infectivity of vaccinated and subsequently infected badgers. From this study, we cannot determine whether a similar reduction in disease progression to that observed in experimental studies was found in the field as no post-mortem data were available. Nevertheless, if that reduction in disease progression does exist, we did not find a concurrent reduction in infectivity. The lack of effect of vaccination on infectivity has implications in terms of the effectiveness of BCG badger vaccination in Ireland (or how much reduction of transmission is achieved by vaccination). The effectiveness of a vaccination programme is the result of both the effect of vaccination on susceptibility and infectivity. Here, as there is no added reduction in transmission due to a reduction in infectivity (one type of indirect effect of vaccination), the total reduction in transmission or effectiveness achieved by vaccination is equal to $\mathrm{VE}_{\mathrm{S}}$.

Once the effectiveness of BCG vaccination was calculated, in order to assess its impact, it was necessary to estimate the ongoing badger to badger transmission. The reproduction ratio for badger to badger transmission under the current control options in Ireland was calculated as 1.22 assuming a badger prevalence of $18 \%$. Based on surveillance data collected from badgers culled as part of an interim badger culling regime in Ireland during 2007 to 2013, an average national prevalence of $14.1 \%$ was estimated (Byrne et al., 2015). However, this includes two partial prevalence estimates (17.7\% for May 2007 to May 2011, and 9.9\% for June 2011 to April 2013), noting that differing methods were used during these periods to differentiate $M$. bovis from non-tuberculous mycobacteria (biochemical tests to May 2011, and PCR techniques subsequently). In this paper, we used $18 \%$ as a conservative prevalence estimate. The formulae used for calculating $\mathrm{R}(0)$ is a basic formulae used to assess transmission in badgers assuming that there is no transmission between cattle and badgers. Although this is likely not the case, we can use this number as an approximation, and conclude that if we were to vaccinate all badgers in Ireland, we would be able to reduce transmission by $59 \%$, with the resulting $R(1)=0.5$, which is substantially below 1 indicating that eradication in badgers would be feasible. Further and by estimating $R(p)$ or the reproduction ratio for a range of vaccine coverages ( $p$ ), we were able to assess what was the minimum 
vaccination coverage necessary to eradicate. The most relevant finding in this manuscript was that in Ireland, vaccination of badgers with a vaccination coverage equal to or higher than $30 \%$ is sufficient to eradicate $M$. bovis infection in badgers, as long as current control strategies also remain in place in both cattle and badgers. The outcomes of this study will have major implications for the control of M. bovis infection in Ireland, not only in badgers but also in cattle. It is important to note that if any or some of the control strategies currently in place have an effect on badger to badger transmission, then modifications to any strategy would have repercussions on the effectiveness of the badger vaccination programme (as the reproduction ratio for badger to badger transmission would change also). For similar reasons, it is not possible to predict the effectiveness of BCG vaccination in badgers in a different country, with different transmission characteristics between badgers.

In this study the infection status of individual badgers was determined by whether or not these badgers tested positive to the Enfer test. Table S1 shows the prevalence of infection as measured by this test. Prevalence values varied between sweeps and zones with prevalence in sweep 2 in zone 1 being much lower $(12.5 \%)$ than that observed in the same zone in sweep $1(31.7 \%)$. The second lowest prevalence observed in the whole study was in zone $\mathrm{C}$ in sweep $5(16.3 \%)$ with the rest being between 20.0-37.8\%. We are not aware of any specific reasons why these prevalence values changed and we assume that these differences are due to randomness. Tuberculosis is a chronic disease with latent and reactivation periods and with serology varying through the different disease stages. If the badger population in the trial differed in terms of disease profile from the 215 badgers in which the test was optimized (i.e. a larger proportion of badgers in a chronic phase in the field trial), the sensitivity of the Enfer test in the trial could be higher or lower than the $25.3 \%$ achieved during test optimization. Indeed, prevalence estimates very much depend on the representativeness of the gold standard panel for the population tested as there is not yet a gold standard test for M. bovis infection in badgers. In a previous study where factors affecting study power were explored, it was shown how high test specificity was paramount (Aznar et al., 2013). Test sensitivity did not play an important role in our ability to detect an effect if BCG vaccination really worked. The fact that we found an effect $\left(\mathrm{VE}_{\mathrm{S}}=59 \%\right)$ suggests that both sensitivity and specificity were sufficiently large and did not affect the study power. The low sensitivity of the test used will also have an effect on incidence and prevalence values and therefore on the beta transmission parameter. We note, however, that the aim of this paper was not to provide true values for these parameters, but rather to use them to estimate $\mathrm{VE}(\mathrm{s})$ by comparing them in the vaccinated and non-vaccinated groups. Badger capture data from this vaccine trial has been previously analysed (Gormley et al., 2017). Two vaccine efficacies were reported from this earlier analysis, one for badgers enrolled during sweeps 1 and $2(\mathrm{VE}=36 \%)$ and other for badgers enrolled during sweeps 3 to $6(\mathrm{VE}=84 \%)$. In that study the direct effect of vaccination was 
estimated by comparing hazard rates of badgers trapped in zone A ( $0 \%$ vaccination coverage) to that of badgers trapped in zone C (100\% vaccination coverage). In addition to the different serological tests used in both studies (incidence in badgers was measured with the BrockTB Stat-Pak lateral flow serology test, (Chambers et al., 2008)), the methodology in which badgers were enrolled for the analysis and the statistical methods used to compute VE estimates were also different. Data from zone $\mathrm{B}$ were not used in the prior analysis, despite badgers from this zone being the ideal population for measuring the direct effect (as vaccinated and non-vaccinated badgers would have been exposed to the same infection pressure).

In a badger vaccine field trial carried out in the UK (Carter et al., 2012), badger setts (rather than individual badgers) were allocated to either vaccine or placebo. From that field study, estimates of the direct effect of BCG vaccination on susceptibility in badgers have been reported with two estimates depending on the diagnostic tests used: $\mathrm{VE}_{\mathrm{S}}=54 \%(95 \% \mathrm{CI}=12-74 \%)$ for the more sensitive test (described as "triple test.") and $\mathrm{VE}_{\mathrm{S}}=76 \%(95 \% \mathrm{CI}=48-89 \%$ for the less sensitive test ("dual test"). Nonetheless due to the study design of choice, separation of the effects of vaccination in susceptibility and infectivity was not possible in either Gormley et al. (2017) or Carter et al. (2012), leading to two biases in the estimate of VEs. Firstly, the indirect effect of BCG vaccination is included in the estimate of $\mathrm{VE}_{\mathrm{S}}$ (although this estimate should only reflect the direct effect of vaccination), and secondly, if the infectivity of vaccinated and non-vaccinated badgers differs, then this difference in infectivity has to be taken into account also when estimating $\mathrm{VE}_{\mathrm{S}}$. In hindsight, and based on our results, we now know that such a difference does not exist $\left(\mathrm{VE}_{\mathrm{I}}=0\right.$ as the coefficient for Fi was not statistically significant). Nonetheless, it is important that this issue is highlighted so it can be considered in the design of future vaccine field trials.

A reduction in $M$. bovis incidence in cubs from vaccinated setts compared to those from non-vaccinated setts was also observed in Carter et al. (2012). In that study, the observed reduction in incidence in cubs is reported as an indirect effect of vaccination. However, it is not possible to distinguish whether this reduction in incidence is due to the indirect effect achieved by a reduction in susceptibility or to a reduction in the infectivity of vaccinated infected badgers compared to nonvaccinated infected ones. Based on our results, the reduction in incidence among cubs was likely due to a reduction in susceptibility of the vaccinated adult badgers in the sett.

\section{Conclusion}

In summary, we have presented a new methodology to estimate both $\mathrm{VE}_{\mathrm{S}}$ and $\mathrm{VE}_{\mathrm{I}}$ providing further knowledge on the biological ways in which BCG vaccination 
works in badgers. We have also presented scientific arguments that support the crucial role of appropriate trial design in order to obtain accurate estimates. Further, we have estimated the impact of vaccination in the current badger transmission Ireland and concluded that a minimum vaccination coverage of $30 \%$ is necessary to achieve eradication of $M$. bovis infection in badgers. As a result of this work, policy makers can now make informed decisions concerning the best strategy or combination of strategies to achieve eradication. These results could also be used to guide the best vaccination route to achieve the minimum vaccine coverage needed.

\section{References}

Ancelet, L.R., Aldwell, F.E., Rich, F.J., Kirman, J.R., 2012. Oral vaccination with lipid-formulated $\mathrm{BCG}$ induces a long-lived, multifunctional $\mathrm{CD} 4(+) \mathrm{T}$ cell memory immune response. PloS ONE 7, e45888.

Aznar, I., McGrath, G., Murphy, D., Corner L.A.L., Gormley, E., Frankena, K., More, S.J., Martin, W., O’Keeffe, J., de Jong M.C.M., 2011. Trial design to estimate the effect of vaccination on tuberculosis incidence in badgers. Vet. Microbiol. 151, 104-111.

Aznar, I., More, S.J., Frankena, K., de Jong, M.C.M., 2013. Estimating the power of a Mycobacterium bovis vaccine trial in Irish badgers. Prev. Vet. Med. 111, $297-$ 303.

Aznar, I., McGrath, G., Murphy, D., Corner, L.A.L., Gormley, E., 2014. Optimising and evaluating the characteristics of a multiple antigen ELISA for detection of Mycobacterium bovis infection in a badger vaccine field trial. PLoS ONE 9, e100139.

Bezos, J., Álvarez, J., Romero, B., de Juan, L., Domínguez, L., 2014. Bovine tuberculosis: historical perspective. Res. Vet. Sci. 97, S3-S4, ISSN 0034-5288, http://dx.doi.org/10.1016/j.rvsc.2014.09.003.

Byrne, A.W., Kenny, K., Fogarty, U., O’Keeffe, J.J., More, S.J., McGrath, G., Teeling, M., Martin, S.W., Dohoo, I.R., 2015. Spatial and temporal analysis of metrics of tuberculosis infection in badgers (Meles meles) from the Republic of Ireland: trends in apparent prevalence. Prev. Vet. Med. 122, 345-354.

Caminiti, A., Pelone, F., LaTorre, G., De Giusti, M., Saulle, R.,Mannocci, A.,Sala, M.,Della Marta, U.,Scaramozzino, P., 2016. Control and eradication of tuberculosis in cattle: a systematic review of economic evidence. Vet. Rec. 179, $70-75$.

Carter, S.P., Chambers, M.A., Rushton, S.P., Shirley, M.D.F., Schuchert, P., Pietravalle, S., Murray, A., Rogers, F., Gettinby, G., Smith, G.C., Delahay, R.J., Hewinson, R.G., McDonald, R.A., 2012. BCG vaccination reduces risk of tuberculosis infection in vaccinated badgers and unvaccinated badger cubs. PLoS One 7, e49833. 
Chambers, M.A., Crawshaw, T., Waterhouse, S., Delahay, R., Hewinson, R.G., Lyashchenko, K.P., 2008. Validation of the BrockTB Stat-Pak assay for detection of tuberculosis in Eurasian Badgers (Meles meles) and influence of disease severity on diagnostic accuracy . J. Clin. Microbiol. 46, 1498-1500.

Chambers, M.A., Rogers, F., Delahay, R.J., Lesellier, S., Ashford, R., Dalley, D., Gowtage, S., Davé, D., Palmer, S., Brewer, J., Crawshaw, T., Clifton-Hadley, R., Carter, S., Cheeseman, C., Hanks, C., Murray, A., Palphramand, K., Pietravalle, S., Smith, G.C., Tomlinson, A., Walker, N.J., Wilson, G.J., Corner, L.A., Rushton, S.P., Shirley, M.D., Gettinby, G., McDonald, R.A., Hewinson, R.G., 2011. Bacillus Calmette-Guerin vaccination reduces the severity and progression of tuberculosis in badgers. Proc. R. Soc. B. 278, 1913-1920.

Corner, L.A.L., O’Meara, D., Costello, E., Gormley, E., 2005. Tuberculosis in badgers: true prevalence, diagnostic methods and epidemiology (poster). Society for Veterinary Epidemiology and Preventive Medicine, Nairn, Scotland.

Corner, L.A.L., Costello, E., Lesellier, S., O’Meara, D., Gormley, E., 2008. Vaccination of European badgers (Meles meles) with BCG by the subcutaneous and mucosal routes induces protective immunity against endobronchial challenge with Mycobacterium bovis. Tuberculosis 88, 601-609.

Corner, L.A.L., Costello, E., O’Meara, D., Lesellier, S., Aldwell, F.E., 2010. Oral vaccination of badgers (Meles meles) with BCG and protective immunity against endobronchial challenge with Mycobacterium bovis. Vaccine 28, 6265-6272.

De Jong, M.C.M., Kimman, T.G., 1994. Experimental quantification of vaccineinduced reduction in virus transmission. Vaccine 12, 761-766.

Diekmann, O., Heesterbeek, J.A.P., Metz, J.A.J., 1990. On the definition and the computation of the basic reproduction ratio $\mathrm{R}_{0}$ in models for infectious diseases in heterogeneous populations. J. Math. Biol. 28, 365-382.

Fine, P.E.M. 1993. Herd immunity: history, theory, practice. Epidemiol. Rev. 15, 265-302.

Gormley, E., N1' Bhuachalla, D., O’Keeffe J., Murphy D., Aldwell F.E., Fitzsimons T., Stanley, P., Tratalos, J.A., McGrath, G., Fogarty, N., Kenny, K., More, S.J., Messam, L.L., Corner, L.A.L., 2017. Oral vaccination of free-living badgers (Meles meles) with Bacille Calmette Guérin (BCG) vaccine confers protection against tuberculosis. PLoS ONE 12, e0168851.

Halloran, M.E., Jr, Longini, I.M., Struchiner, C.J., 1999. Design and interpretation of vaccine field studies. Epidemiol. Rev. 21, 73-88.

Langer, A.J. and LoBue, P.A., 2014. Public health significance of zoonotic tuberculosis caused by the Mycobacterium tuberculosis complex, in zoonotic tuberculosis: Mycobacterium bovis and other pathogenic mycobacteria, Third Edition (eds C. O. Thoen, J. H. Steele and J. B. Kaneene), John Wiley \& Sons, Inc, Chichester, UK.

Lesellier, S., Corner, L.A.L., Costello, E., Lyashchenko, K., Greenwald, R., Esfandiari, J., Singhd, M., Hewinsone, R.G., Chambers, M., Gormley, E., 2009. 
Immunological responses and protective immunity in BCG vaccinated badgers following endobronchial infection with Mycobacterium bovis. Vaccine 27, 402409.

Lesellier, S., Palmer, S., Gowtage-Sequiera, S., Ashford, R., Dalley, D., Davé, D., Weyer, U., Salguero, F.J., Nunez, A., Crawshaw, T., Corner, L.A.L., Hewinson, R.G., Chambers, M.A., 2011. Protection of Eurasian badger (Meles meles) from tuberculosis after intra-muscular vaccination with different doses of BCG. Vaccine 29, 3782-3790.

Longini, I.M., Jr, Sagatelian, K., Rida, W.N., Halloran, M.E., 1998. Optimal vaccine trial design when estimating vaccine efficacy for susceptibility and infectiousness from multiple populations. Stat. Med. 17, 1121-1136.

Moerman, A., Straver, P.J., de Jong, M.C., Quak, J., Baanvinger, T., van Oirschot J.T., 1993. A long-term epidemiologic study of bovine viral diarrhea infections in a large herd of dairy cattle. Vet. Rec. 132, 622-626.

More, S.J., Good, M., 2006. The tuberculosis eradication programme in Ireland: a review of scientific and policy advances since 1988. Vet. Microbiol. 112, 239251.

Murphy, D., Gormley, E., Costello, E., O’Meara, D., Corner L.A.L., 2010. The prevalence and distribution of Mycobacterium bovis infection in European badgers (Meles meles) as determined by enhanced post mortem examination and bacteriological culture. Res. Vet. Sci. 88, 1-5.

Murphy, D., Costello, E., Aldwell, F.E., Lesellier, S., Chambers, M.A., Fitzsimons, T., Corner, L.A.L., Gormley, E., 2014. Oral vaccination of badgers (Meles meles) against tuberculosis: Comparison of the protection generated by BCG vaccine strains Pasteur and Danish. Vet. J. 200, 362-367.

Stegeman, A., van Nes, A., de Jong, M.C.M., Bolder, F., 1995. Assessment of the effectiveness of vaccination against pseudorabies in finishing pigs. Am. J. Vet. Res. 56, 573-578.

Stephens, D.S., 2008. Vaccines for the unvaccinated: protecting the herd. [Editorial commentary]. J. Infect. Dis. 197, 643-645.

Whelan, C., Shuralev, E., O'Keeffe, G., Hyland, P., Kwok, H.F., Snoddy, P., O’Brien, A., Connolly, M., Quinn, P., Groll, M., Watterson, T., Call, S., Kenny, K., Duignan, A., Hamilton, M.J., Buddle, B.M., Johnston, J.A., Davis, W.C., Olwill, S.A., Clarke., J., 2008. Multiplex immunoassay for serological diagnosis of Mycobacterium bovis infection in cattle. Clin. Vaccine Immunol. 15, 18341838.

Whelan, C., Whelan, A.O., Shuralev, E., Kwok, H.F., Hewinson, G., Clarke, J., Vordermeier, H.M., 2010. Performance of the Enferplex TB assay with cattle in Great Britain and assessment of its suitability as a test to distinguish infected and vaccinated animals. Clin. Vaccine Immunol. 17, 813-817. 


\section{Quantification of Mycobacterium bovis transmission in a badger vaccine field} trial

Supplementary material

- $\quad$ 1. Initial derivations and model building

The derivations to support the estimations of the transmission parameters have already been published (12). In summary, and before considering vaccination, we showed how the number of new infections among all susceptible badgers $(C)$ at the end of the time interval between two sweeps can be modelled using a binomial distribution with parameters $\mathrm{S}$ and $p$ (where $\mathrm{S}$ is the number of susceptible badgers at the beginning of the time interval and $p=\left(1-e^{-\beta * I * \frac{\Delta t}{N}}\right)$ is the probability that each of these badgers will become infected during that time interval). The expected number of new cases is then equal to:

$$
E(C)=S *\left(1-e^{-\beta * I * \frac{\Delta t}{N}}\right)
$$

Then, as the number of new cases among susceptibles (C) can be obtained through serology, the transmission parameter $\beta$ could be estimated by using a Generalized Linear Model (GLM) with a complementary-log-log link function, a binomial error function, with binomial total S and offset $\ln \left(I * \frac{\Delta t}{N}\right)$.

We followed then by deriving new formulae that took into account vaccination, showing how the number of new infections among susceptibles at the end of each sweep could be estimated separately for vaccinated and non-vaccinated badgers as:

$$
\begin{aligned}
& E\left(C_{V}\right)=S *\left(1-e^{-e^{k_{0}+k_{1} * F_{1} * \text { Prev } * \Delta t}}\right) \\
& \text { (where } \beta_{\mathrm{UV}}=\operatorname{Exp}\left[\mathrm{k}_{0}\right] \text { and } \beta_{\mathrm{VV}}=\operatorname{Exp}\left[\mathrm{k}_{0}+\mathrm{k}_{1}\right] \text { ) and } \\
& E\left(C_{U}\right)=S *\left(1-e^{-e^{k_{0}+k_{1} * F_{1} * \text { Prev } * \Delta t}}\right)
\end{aligned}
$$

(where $\beta_{\mathrm{UU}}=\operatorname{Exp}\left[\mathrm{k}_{0}\right]$ and $\beta_{\mathrm{VU}}=\operatorname{Exp}\left[\mathrm{k}_{0}+\mathrm{k}_{1}\right]$ ), respectively.

Prev being the prevalence $(\mathrm{I} / \mathrm{N})$ and $\mathrm{Fi}$ the fraction of infected vaccinated badgers in the zone where the badger was trapped at the beginning of the time interval. Here, as $\mathrm{C}_{\mathrm{V}}$ and $\mathrm{C}_{\mathrm{U}}$ are known, all four betas can be calculated by using a GLM similar to the one described above.

In this paper, by assuming separable mixing (and as the vaccination status of each individual badger is available), we can then modify the GLM model introducing vaccination status of the recipient also as one of the explanatory variables allowing us to estimate all the transmission rate parameters from the total population:

$$
\operatorname{clog} \log (C)=\beta_{0}+\beta_{1, B} Z_{B}+\beta_{1, C} Z_{C}+\beta_{2} V s+\beta_{3} F i
$$

A manual model building was carried out to select the final model; as the number of predictors in the maximum model was small, all possible combination of predictors were examined. The model including all three predictors: zone, vaccination status 
and fraction of infected vaccinated badgers was selected based on the lowest AIC $(\mathrm{AIC}=490.2)$.

- 2. Descriptive results

Table S1. Number and percentage of positive trappings to the Enfer chemiluminescent multiplex ELISA at each sweep, overall and by zone.

\begin{tabular}{|c|c|c|c|c|c|c|c|c|}
\hline \multirow[b]{3}{*}{ Sweep } & \multicolumn{6}{|c|}{ Zone } & \multicolumn{2}{|c|}{ Overall } \\
\hline & \multicolumn{2}{|r|}{ A } & \multicolumn{2}{|r|}{ B } & \multicolumn{2}{|r|}{ C } & & \\
\hline & No & \% Positive & No & \% Positive & No & \% Positive & No & \% Positive \\
\hline 1 & 120 & 31.67 & 63 & 19.05 & 115 & 23.48 & 298 & 25.84 \\
\hline 2 & 40 & 12.5 & 22 & 22.73 & 40 & 30 & 102 & 21.57 \\
\hline 3 & 101 & 34.65 & 46 & 23.91 & 85 & 31.76 & 232 & 31.47 \\
\hline 4 & 99 & 25.25 & 49 & 36.73 & 60 & 35 & 208 & 30.77 \\
\hline 5 & 105 & 26.67 & 46 & 26.09 & 98 & 16.33 & 249 & 22.49 \\
\hline 6 & 90 & 37.78 & 67 & 35.82 & 98 & 30.61 & 255 & 34.51 \\
\hline 7 & 117 & 27.35 & 65 & 36.92 & 80 & 27.5 & 262 & 29.77 \\
\hline 8 & 59 & 37.29 & 30 & 33.33 & 63 & 32.81 & 153 & 34.64 \\
\hline
\end{tabular}

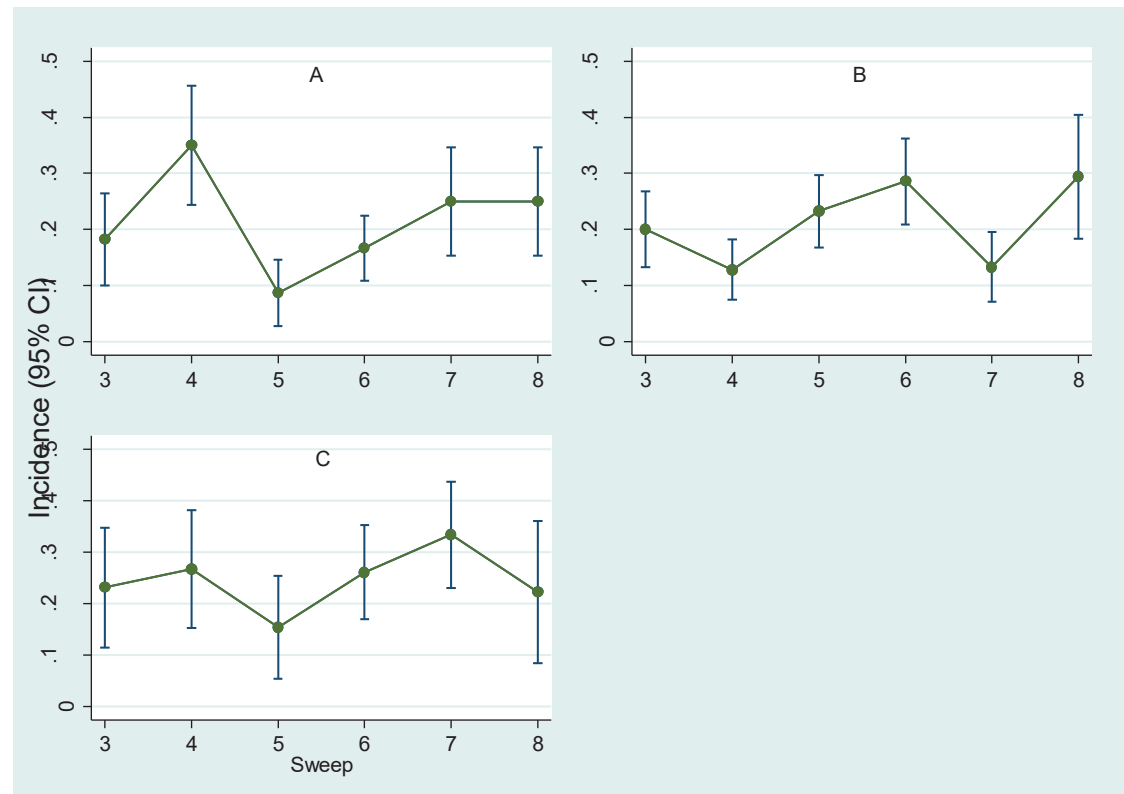

Figure S1. Incidence per sweep (estimated as number of new cases divided by the total number of susceptible badgers per sweep) and exact confident intervals (95\% $\mathrm{CI})$ presented by trial zone. 


\section{Supporting modelling exercises}

Table S2. Generalized Linear Model results showing coefficient, p-value and 95\% confidence interval for the explanatory variable (vaccination status) and constant when the model was fitted in data from zone B only.

\begin{tabular}{lccc}
\hline Variable & Coef & p-Value & $(\mathbf{9 5 \%}$ CI) \\
\hline Constant & -5.97 & $<0.001$ & -5.97 to -4.90 \\
Vs & $\mathbf{- 0 . 7 9}$ & $\mathbf{0 . 0 5 9}$ & $\mathbf{- 1 . 6 0 ~ t o ~} \mathbf{- 0 . 0 3}$ \\
\hline
\end{tabular}

Table S3. Generalized Linear Model results showing coefficient, p-value and 95\% confidence interval for all three explanatory variables (and constant): zone, vaccination status and the fraction of infected vaccinated badgers when the model was fitted in data from zones B and C only.

\begin{tabular}{|c|c|c|c|c|}
\hline \multicolumn{2}{|l|}{ Variable } & Coef & p-Value & $(95 \%$ CI $)$ \\
\hline \multicolumn{2}{|l|}{ Constant } & -5.55 & $<0.001$ & -6.09 to -5.00 \\
\hline \multicolumn{5}{|l|}{ Zone } \\
\hline \multicolumn{2}{|r|}{ B } & \multicolumn{3}{|c|}{ Reference } \\
\hline & $\mathrm{C}$ & 0.78 & 0.832 & -0.64 to 0.79 \\
\hline Vs & & -0.92 & 0.029 & -1.76 to -0.09 \\
\hline $\mathrm{Fi}$ & & 1.67 & 0.065 & -0.11 to 3.45 \\
\hline
\end{tabular}

- 4. Supplementary note on omitted data

On reconciliation of project databases at the completion of the trial, it was noted that 70 samples had not been tested by Enfer, due to a procedural error. These samples belonged to badgers that had been trapped in sweep 2 and were either trapped in sweep 1 or later on at subsequent sweeps. The samples were equally distributed between all three zones. The samples were also equally distributed in terms of vaccination status of the badger they belonged to. The missing samples represented between $9.0 \%$ and $10.7 \%$ of our entries for statistical analysis (depending on the infection status). The reduction in power was not considered to be a major constraint as the statistical analysis, in the absence of these 70 samples, showed significant results for $\mathrm{VE}_{s}$. Additionally, even with the inclusion of these 70 samples, the estimates of $\mathrm{VE}_{\mathrm{I}}$ would never be significantly above zero. 

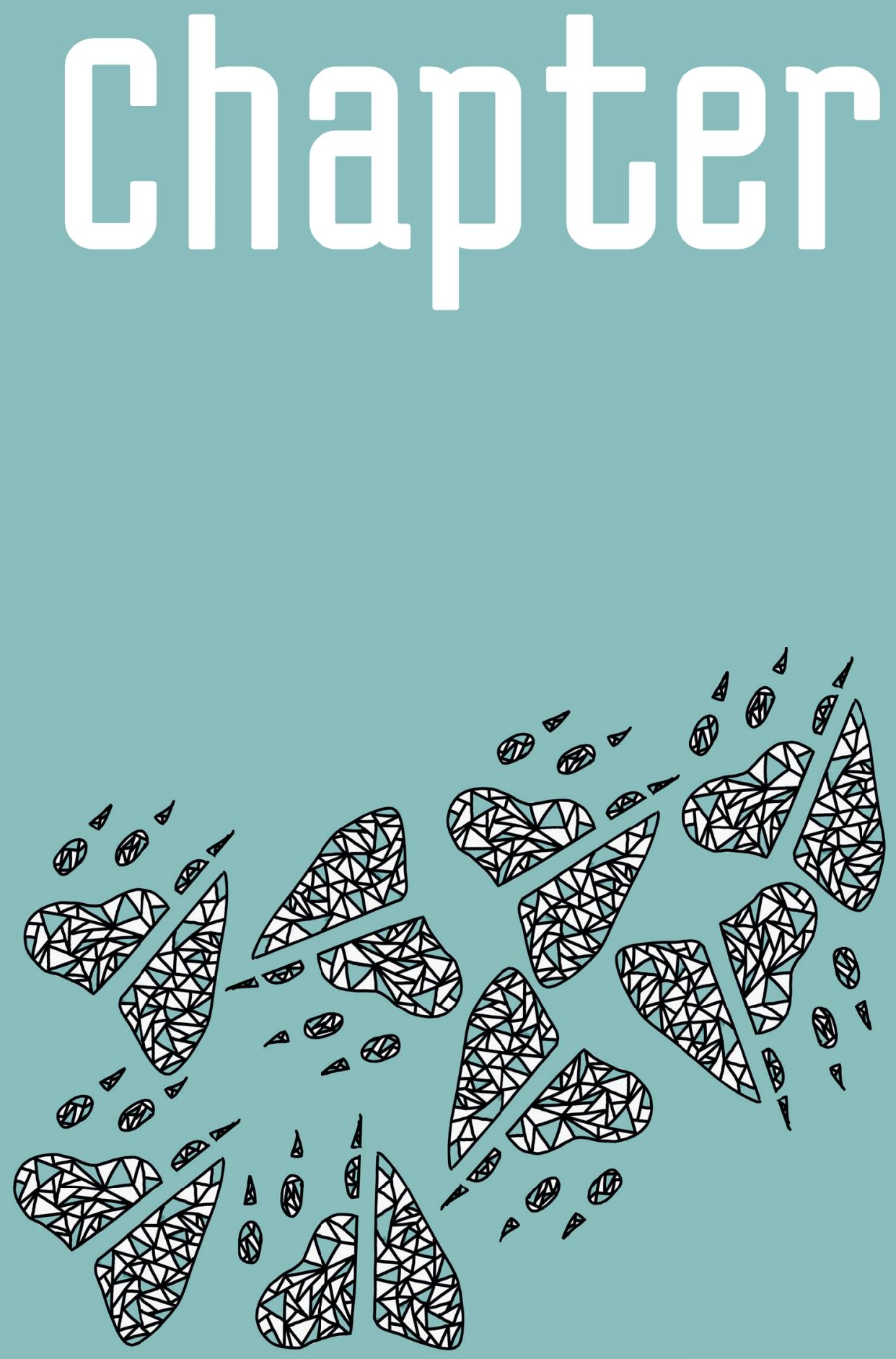


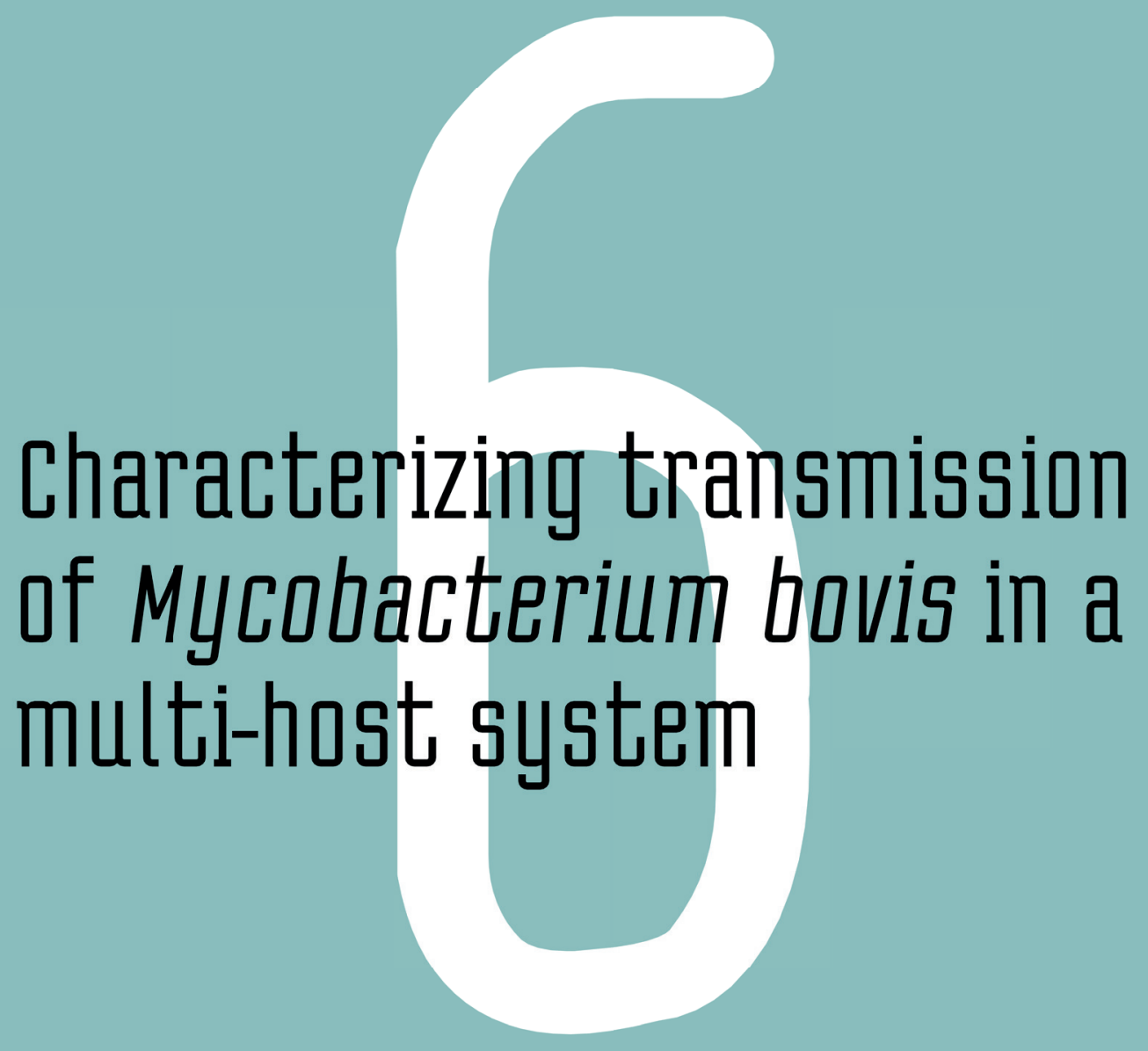

Aznar, I. , Frankena, K. , More, S.J. , Clegg, T.A. , McGrath, G. , D'Keeffe, J., Byrne, A.W. , de Jong, M.C.M. 
Chapter 6

\section{Characterizing transmission of Mycobacterium bovis in a multi- host system}

Aznar, I., Frankena, K., More, S.J., Clegg, T.A., McGrath, G., O’Keeffe, J., Byrne, A.W., de Jong, M.C.M.

Submitted to PNAS 


\begin{abstract}
In Ireland, in spite of a comprehensive control/eradication programme for Mycobacterium bovis, tuberculosis in cattle (bTB) has remained endemic in cattle at a low prevalence for the last 20 years. Badgers (Meles meles) are considered to be the main wildlife reservoir for M. bovis infection in Ireland and Great Britain. In order to select strategies that allow eradication of the infection in cattle, knowledge of the quantitative role of each of these two host species in the transmission of $M$. bovis is essential. Here, we derive algorithms that show how the Next Generation Matrix (NGM) and the reproduction ratio $(R)$ for the cattle-badger system can be calculated from prevalence data and the number of cattle herds and badgers, collected at the endemic steady state. By exploring the system at the endemic equilibrium, we can set bounds for the possible transmission scenarios between cattle and badgers in Ireland and demonstrate that for almost all these scenarios (except when all or almost all infection in cattle is solely caused by cattle), BCG vaccination of badgers in addition to current controls could successfully achieve eradication of M. bovis infection in the system.
\end{abstract}




\section{Introduction}

Many animal pathogens are able to infect multiple hosts. This ability has been shown to be associated with a larger risk of disease emergence in humans (Haydon, 2002). In Ireland, Mycobacterium bovis, the infectious agent causing bovine tuberculosis (bTB) in cattle and other bovids, is a good example of a multi-host pathogen with at least one other main reservoir of infection, the Eurasian badger (Meles meles). M. bovis infection in humans, which are considered to be a spill-over host (Gormley and Corner, 2013), is uncommon in Ireland (2.5\% of the culture isolates from all tuberculosis diagnoses) as a result of disease controls in cattle and other public health measures (FSAI, 2017). Most cases of M. bovis infection in humans are linked to infection in cattle either following consumption of unpasteurized milk and milk products or, less likely, following direct contact with infected animals (FSAI, 2017). A sound understanding of the dynamics of M. bovis in the two main reservoirs (cattle and badgers) is critical, to address public health concerns, and to reduce the economic burden on farmers and other members of society of ongoing measures to control and eradicate the infection in cattle.

Estimating transmission parameters can help in predicting population dynamics. One important parameter is the basic reproduction ratio, $R_{0}$, which is defined as the average number of new cases caused by one typical infectious individual in a totally susceptible population. $R$, or reproduction ratio when the population might not be fully susceptible, is a very important parameter in population dynamics of infectious diseases as it can be used to summarise dynamic behaviour of an infection in a host population with just one single value (Kermack and McKendrick, 1927; Diekmann et al., 1990, 2010; Fulford et al., 2002). An epidemic can only be sustained if $R>1$, therefore the efficacy of control measures can be assessed based on whether or not they are capable of reducing $R$ below one.

In a single host system $R$ for the system can be estimated, by observing the endemic steady state, as the inverse of the fraction of susceptible hosts $\left(R=\mathrm{N} / S^{*}\right)$ (Keeling and Rohani, 2008 for a textbook explanation). Note that an asterisk is used to denote parameters at the endemic equilibrium. In the case of a homogeneous population consisting of multiple hosts, $R$ can still be calculated by adding the number of susceptibles of each host type at the endemic equilibrium and dividing this number by the total population. However, this calculation is not applicable when some form of heterogeneity (either in infectivity or susceptibility) exists between the hosts. In such situations, $R$ estimations are more challenging.

One way of estimating $R$ for the cattle-badger system would be to build a Next Generation Matrix (NGM), a concept explained originally by Diekmann et al. (1990), and further referred by other authors (Fulford et al., 2002; Heesterbeek and Roberts, 1995; Heesterbeek, 2000), which defines the dynamics of bTB in this 
system. $R$ can then be determined mathematically as the dominant eigenvalue of this NGM. We could design a 2 x $2 \mathrm{NGM}(\boldsymbol{K})$ made of four terms: $\boldsymbol{K}_{c c}, \boldsymbol{K}_{b c}, \boldsymbol{K}_{c b}$ and $\boldsymbol{K}_{b b}$. Each of the four terms of this matrix being a matrix in itself that describes transmission between/within host species, with indices " $c$ " and " $b$ " referring to the host species cattle and badgers, respectively, and the direction of the transmission determined by the location of these indices; i.e. $\boldsymbol{K}_{b c}$ is the matrix that describes the transmission of $M$. bovis from badgers to cattle. Then, $R$ for the system is the dominant eigenvalue of $\boldsymbol{K}$.

A disadvantage of this approach concerns parameterization of the models that define each of these matrices. Estimating interspecies transmission rates between cattle and badgers has never been described, resulting in the introduction of uncertainty in final model outputs (Godfray et al., 2013). Then again, computation of the NGM for the endemic steady state for the two species can also be done directly if specific assumptions are made regarding the relative transmission between/within these two host types. The NGM that defines transmission in the cattle-badger system in Ireland can be easily estimated when assuming no interspecies transmission (to be called disjunct mixing). In this case, both $R_{b c}$ (dominant eigenvalue of $\boldsymbol{K}_{b c}$ ) and $R_{c b}$ (dominant eigenvalue of $\boldsymbol{K}_{c b}$ ) will be equal to 0 , with the intraspecies transmission rates being estimated independently from each of the species prevalence respectively. $R$ for the system is then equal to the largest real value of either $R_{c c}$ (dominant eigenvalue of $\boldsymbol{K}_{c c}$ ) or $R_{b b}$ (dominant eigenvalue of $\boldsymbol{K}_{b b}$ ). It is more difficult though to estimate the NGM in a situation where cattle and badgers do interact to some extent.

In this paper, by exploring the cattle-badger system at the endemic equilibrium, we have set the theoretical bounds that frame all plausible transmission scenarios in this system: i.e. all possible NGMs given the observed endemic steady state data for Ireland. Then, based on the arguments presented, we have estimated three extreme NGMs that are the nodes of a transmission matrix triangle that contains all possible transmission scenarios (NGMs) for M. bovis in Ireland. Aside, we have derived new equations that allow estimation of any NGM under the assumption of separable mixing and also showed how series of NGMs that do not follow this assumption can be calculated. We have illustrated this by calculating several plausible NGMs that assume different types of interaction between the two species. All derived equations only require knowledge of endemic prevalence data and total number of individuals in each of the species. The effect on $R$ achieved by BCG badger vaccination is estimated in all plausible transmission scenarios; a small number of unlikely transmission scenarios where additional badger control measures cannot achieve eradication of M. bovis from the system are explained. Finally, the impact of adding badger vaccination to the current $M$. bovis eradication strategy in Ireland is discussed. 


\section{Results and discussion}

Theoretical boundaries

By observing the characteristics of matrices at the endemic steady state and taking into account biological properties of the cattle and badger system in Ireland, we have set the theoretical boundaries for all plausible Jacobian matrices $\left(M^{\prime}\right)$ in the endemic steady state, and subsequently for all NGMs at the disease free equilibrium (NGM), that could describe transmission in this system. At the endemic equilibrium, all plausible $M$ 's that could describe this transmission will have a maximum eigenvalue equal to 1 (i.e. the reproduction ratio at the endemic equilibrium or dominant eigenvalue is 1) and will share a unique eigenvector $(e)$. This eigenvector $e$ is observed at the endemic equilibrium as the relative frequency of cattle and badgers among the infected individuals. So for all $M^{\prime} \mathrm{s}$ :

$$
M^{\prime} * e=e * \mathbf{1}=e
$$

Because of the biological interpretation, it follows that $M^{\prime}$ matrices can only have non-negative elements $(E)$, so for a matrix:

$$
M^{\prime}=\left(\begin{array}{cc}
E_{c c}^{\prime} & E_{b c}^{\prime} \\
E_{c b}^{\prime} & E_{b b}^{\prime}
\end{array}\right) \text {, then } E_{c c}^{\prime}, E_{b c}^{\prime}, E_{c b}^{\prime}, \text { and } E_{b b}^{\prime} \geq 0,
$$

where $E_{c c}^{\prime}$ is the endemic transmission for cattle herd to cattle herd, $E_{b c}^{\prime}$ is the endemic transmission for badger to cattle herd, $E_{c b}^{\prime}$ is the endemic transmission for cattle herd to badger and $E_{b b}^{\prime}$ the endemic transmission for badger to badger.

In the cattle-badger system, the prevalence of $M$. bovis infection in cattle herds and badgers are $>0$ indicating that at least one element on each row of the matrix that describes this transmission is $>0$. Therefore, all plausible matrices in the cattlebadger system will be bounded by matrices with two elements equal to 0 , as 0 is the minimum value that the other two elements can take. Although there are 6 possible combinations for a $2 \times 2$ matrix with two elements equal to 0 , three of these combinations are not biologically plausible. As cattle herds are infected, the infection must have originated either from other cattle herds $\left(E_{c c}^{\prime}>0\right)$ or from badgers $\left(E_{b c}^{\prime}>\right.$ 0 ) implying that a matrix of the type $M^{\prime}=\left(\begin{array}{cc}0 & 0 \\ E_{c b}^{\prime} & E_{b b}^{\prime}\end{array}\right)$ is not plausible. Following the same logic for badgers, we can conclude that a matrix of the type $M^{\prime}=\left(\begin{array}{cc}E_{c c}^{\prime} & E_{b c}^{\prime} \\ 0 & 0\end{array}\right)$ is not plausible either. Further the possibility that cattle herds do not infect other cattle herds and badgers do not infect other badgers is not considered biologically valid: i.e. $M^{\prime}=\left(\begin{array}{cc}0 & E_{b c}^{\prime} \\ E_{c b}^{\prime} & 0\end{array}\right)$ is not a valid matrix. Thus, there are three possible $M^{\prime}$ s that bound all plausible transmission scenarios for the cattle-badger system: $M^{\prime}=$ $\left(\begin{array}{cc}E_{c c}^{\prime} & 0 \\ 0 & E_{b b}^{\prime}\end{array}\right), M^{\prime}=\left(\begin{array}{cc}E_{c c}^{\prime} & 0 \\ E_{c b}^{\prime} & 0\end{array}\right)$, and $M^{\prime}=\left(\begin{array}{cc}0 & E_{b c}^{\prime} \\ 0 & E_{b b}^{\prime}\end{array}\right)$. As $M^{\prime} * e=e$, given two known matrix elements, the other two elements can be ascertained (as this relationship 
yields two equations and only two unknowns). To move from these Jacobian matrices $M$ 's estimated at the endemic equilibrium to the NGMs estimated at disease free equilibrium, these three matrices need to be adjusted to account for the fact that at the disease free equilibrium the total population is susceptible. The elements of these matrices, when divided by the fraction of susceptible, will become the partial reproduction ratios estimated at the endemic equilibrium: $R_{c c}^{\prime}, R_{c b}^{\prime}, R_{b c}^{\prime}$ and $R_{b b}^{\prime}$. These matrices will also have to be adjusted to reflect the right distribution of the different host types, with the resultant matrices being made of the partial reproduction ratios estimated at the disease free equilibrium: $R_{c c}, R_{c b}, R_{b c}$ and $R_{b b}$. The methodology to do these corrections is presented in section 3.2.2.

The three resultant NGMs calculated in this way can be represented then as the vertices of a triangle placed on a plane, with these three vertices, the lines connecting these vertices and the area within the triangle representing all possible NGMs in the cattle-badger system in Ireland (Figure 1).

In this triangle, the NGM obtained when no interspecies interaction is assumed (disjunct mixing) or $N G M=\left(\begin{array}{cc}R_{c c} & 0 \\ 0 & R_{b b}\end{array}\right)$, bounds inter-species transmission at the lower end with $R_{c b}=R_{c b}=0$ and is presented as node 1 in the transmission triangle (Figure 1). The other two matrices represent transmission scenarios where the infection is solely originated from either cattle $\left(N G M=\left(\begin{array}{ll}R_{c c} & 0 \\ R_{c b} & 0\end{array}\right)\right)$ or badgers $\left(N G M=\left(\begin{array}{ll}0 & R_{b c} \\ 0 & R_{b b}\end{array}\right)\right.$ ) and are shown as nodes 2 and 3, respectively, in the transmission triangle. From node 1, two lines, one towards node 2 and one towards node 3 can be drawn. These two connecting lines between the three nodes will consist of NGMs that have one element equal to 0 , i.e. the NGMs on the line between node 1 and node 2 will be of the form $\left(\begin{array}{cc}R_{c c} & 0 \\ R_{c b} & R_{b b}\end{array}\right)$ while the NGMs on the line between node 1 and 3 will be of the form $\left(\begin{array}{cc}R_{c c} & R_{b c} \\ 0 & R_{b b}\end{array}\right)$. These two lines bound the space of possible NGMs as the 0 element would become negative beyond these lines (see Figure 1).

If we assume that infectivity and susceptibility intraspecies is always larger than the infectivity and susceptibility interspecies, we can define the third line of the triangle (line that joins nodes 2 and 3 ) as a line made of NGMs under the separable mixing scenario. In this line, many combinations of susceptibility and infectivity are possible, with nodes 2 and 3 representing transmission scenarios with maximum infectivity of cattle and badgers respectively. It is important to note that although the interspecies susceptibility and infectivity at this line will never be larger than that of the intraspecies, some of the susceptibility-infectivity combinations could result in 
NGMs where the interspecies transmission parameter is larger than the intraspecies equivalent.

The two axes of the plane where the transmission triangle lies are therefore: $y$-axis: a vertical axis measuring the relative contribution of cattle to the total number of infections in the system. This axis has a scale from 0 to 1 based on the formula: $R_{c c} /\left(R_{c c}+R_{b b}\right)$, and x-axis: a horizontal axis that indicates the level of interaction between the two species. This axis is also measured from 0 to 1 , with 0 indicating maximal interaction (separable mixing) and 1 none. The level of interaction will be measured by $x$. In order to scale the axis from 0 to 1 , the interspecies endemic transmission parameters will be multiplied by $(1-x)$ as shown in the following matrix: $M^{\prime}=\left(\begin{array}{cc}E_{c c}^{\prime} & (1-x) E_{b b}^{\prime} \\ (1-x) E_{c c}^{\prime} & E_{b b}^{\prime}\end{array}\right)$. For this $M^{\prime}$ series as well as for other series types, the matrices will be resolved following the methodology shown in section 3.2.2. For that, the two interspecies endemic transmission parameters will be formulated as a function of their intraspecies equivalent.

Derivations to estimate any of the NGMs along the y-axis (separable mixing) are covered in section 3.2.1 while derivations to estimate any of the NGMs within the triangle as well as those on the lines joining nodes 1 and 2 and nodes 1 and 3 are shown in 3.2.2.

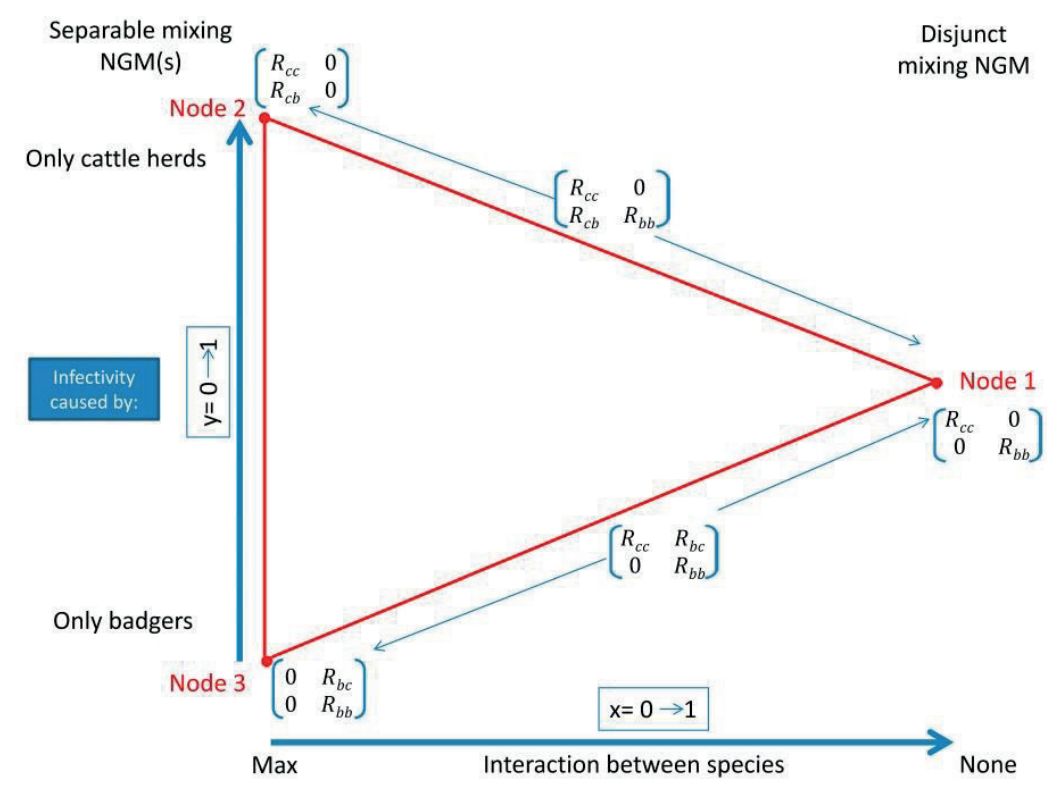

Figure 1. Theoretical transmission triangle delimited by nodes 1, 2 and 3.

$\underline{\text { NGMs results }}$ 
Here the results for the three NGMs that form the nodes in the theoretical transmission triangle are presented. Further, we present the results for two other NGMs contained within this triangle, NGM A and NGM B.

Under the assumption of disjunct mixing (no interspecies transmission), the reproduction ratio for $M$. bovis infection in cattle herds, badgers and the system, respectively, were estimated as:

$$
\begin{gathered}
R_{c c}=\frac{1}{1-\text { prev cattle herds }}=\frac{1}{1-0.063}=1.07 \\
R_{b b}=\frac{1}{1-\text { prev badgers }}=\frac{1}{1-0.14}=1.16
\end{gathered}
$$

with $R=\max \left(R_{c c}, R_{b b}\right)=1.16$

The NGM resulting from these results represents node 1 in the transmission triangle shown in Figure 1 and 2 ( $R_{b c}=R_{c b}=0$ as there is no interspecies transmission). To estimate the partial reproduction ratios, we used a prevalence of $M$. bovis infection in cattle herds and badgers of $6.3 \%$ and $14 \%$, respectively, and a total number of 72,228 cattle herds and 63,000 badgers (see Material and Methods, 3.1).

Under the assumption of separable mixing and using the combined prevalence in both species, the reproduction ratio for the cattle-badger system was estimated from endemic equilibrium data as $\frac{1}{f_{S}^{T O T}}\left(3.2 .1\right.$, section $a$.). Here, $f_{S}^{T O T}$ represents the fraction of susceptible cattle herds and badgers out of the total population (cattle herds and badgers) at the endemic equilibrium. The "reproduction ratio" of the system from the endemic equilibrium (as opposite to the real reproduction ratio) was then calculated using equation (4) as $R=1.11$.

Assuming separable mixing, we also estimated the Jacobian matrix for the cattle and badger system at the endemic equilibrium (3.2.1, section $b 1)$ ). Using equations (5) and (6), and as the susceptibility of badgers can be estimated as $y_{b}=\frac{\gamma_{b}}{\bar{\gamma}_{t}}=\frac{f_{b}^{l}}{f_{b}^{S}}$ (3.2.1, section $c$.), we estimated $R_{c c}^{\prime}=0.38$ and $R_{b b}^{\prime}=0.73$ for a scenario where the relative infectivity of cattle and badgers are the same $\left(x_{c}=x_{b}=1\right)$. Similarly, both partial reproduction ratios were estimated for two extreme scenarios, one where all the infectivity came from cattle herds $\left(R_{c c}^{\prime}=1.11\right.$ and $\left.R_{b b}^{\prime}=0\right)$ and one where all the infectivity came from badgers $\left(R_{c c}^{\prime}=0\right.$ and $\left.R_{b b}^{\prime}=1.11\right)(3.2 .1$, section $\left.b 1)\right)$. In all three scenarios ${ }^{\prime}=R_{c c}^{\prime}+R_{b b}^{\prime}=1.11$, as a result of the separable mixing assumption.

For a homogeneous population, the $R^{\prime}$ estimated at the endemic steady state is indeed a correct estimate of the real $R$. However, for a system such as the cattle-badger system where there is potential heterogeneity between cattle herds and badgers in terms of their susceptibility to $M$. bovis infection, this $R^{\prime}$ is no longer a correct estimate for the real reproduction ratio (Biemans et al., 2017). When an infectious agent is introduced into an infection free population with more than one host type 
(such as the cattle-badger system), if the susceptibility of the host types involved is the same, the depletion of susceptible individuals would occur at the same rate in the different host types (i.e. cattle and badgers). If susceptibility of the cattle herds and badgers differ, then the depletion of individuals of the most susceptible type would occur more quickly than that of the less susceptible type. Thus the relative attack rate (a combination of frequency of occurrence and susceptibility) of the more susceptible type will decrease as we approach the endemic equilibrium. Below, we present the results obtained when the NGM at the disease free equilibrium and $R_{0}$ are estimated as an extrapolation from the Jacobian matrix at the endemic equilibrium, taking into account the difference between the rate of depletion among cattle herds and among badgers (due to differences in terms of their susceptibility). Two extreme NGMs assuming separable mixing were calculated. If we assume all infectivity comes from cattle herds (Material and Methods 3.2.1, section b2), then $R=R_{c c}=1.07, R_{c b}=2.25, R_{b c}=0$ and $R_{b b}=0$ (NGM presented in node 2 in Figure 1 and 2). Assuming all infectivity originates from badgers $R=R_{b b}=1.16, R_{b c}=$ $0.55, R_{c b}=0$ and $R_{c c}=0$ (NGM shown in node 3 in Figure 1 and 2). Another NGM assuming separable mixing and equal infectivity of cattle herds and badgers was estimated using equations (7), and (8) respectively as $R_{b b}=R_{b c}=0.77$ and $R_{c c}=$ $R_{c b}=0.36$ (NGM A in Figure 2), and its reproduction ratio was also estimated using equation (9) as $R=0.77+0.36=1.13$.

In the derivations presented in section 3.2.2, we show how all possible NGMs within the transmission triangle can be estimated. The general formula based on the Irish data for calculating the NGM at the disease free equilibrium $M$ from $M^{\prime}$ is:

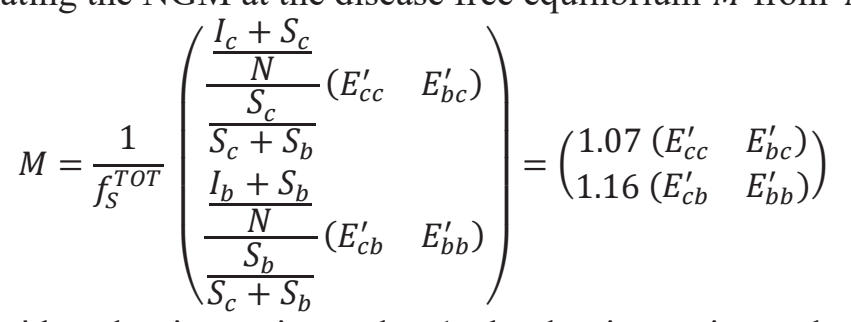

Note that as $M^{\prime}$ has dominant eigenvalue 1, the dominant eigenvalue of the NGM matrix $M$ has to be between 1.07 and 1.16. The eigenvector in the endemic equilibrium is $(c, b)=(0.340332,0.659668)$.

To illustrate, we can look at node 2 (as an example of a separable mixing matrix); here, the matrix obtained by solving the two equations system as indicated in 3.2.2

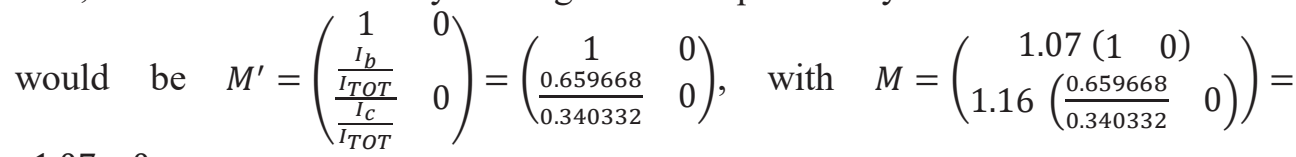
$\left(\begin{array}{ll}1.07 & 0 \\ 2.25 & 0\end{array}\right)$ with $R=1.07$. These are the same values we obtained above when using 
the methodology described in 3.2.1. In a similar way, other NGMs that do not assume separable mixing can also be calculated. Here, we estimated the NGM for a matrix $M^{\prime}=\left(\begin{array}{cc}E_{c c}^{\prime} & x E_{b b}^{\prime} \\ x E_{c c}^{\prime} & E_{b b}^{\prime}\end{array}\right)$ that assumes very low interspecies transmission ( 0.06 times that of the intra-species transmission, i.e. $x=0.06$ ) as an example of a matrix opposed to the maximum interspecies transmission observed in the case of separable mixing. The results obtained were: $R_{b c}=0.07, R_{c b}=0.06, R_{c c}=0.95$ and $R_{b b}=1.13$ with $R=1.15$ (NGM B in Figure 2).

Figure 2 below presents the results for three extreme NGMs and $R$ s that frame all possible matrices that can describe transmission in cattle herds and badgers in Ireland. Also NGMs A and B, are shown as an example of NGMs contained within the triangle. The transmission triangle is presented on a plane with the $y$-axis indicating the origin of the infectivity (cattle herds and/or badgers), and the x-axis indicating the level of interaction between the two species. Matrix A is the NGM obtained when assuming separable mixing and equal infectivity of cattle herds and badgers. In this triangle, as we move up from this matrix, the infectivity of cattle herds increases while the infectivity of badgers decreases, and the opposite occurs as we move down. This matrix also assumes maximum interaction between the two species, with this interaction decreasing as we move towards the right hand side of the triangle. Matrix B is also presented within the transmission triangle and has been located close to node 1 , as very small interspecies transmission is assumed. Note that this NGM is similar to the matrix assumed by Brooks-Pollock \& Danon (2017) based on model assumptions in Brooks-Pollock \& Wood (2015), apart from the essential asymmetry in transmission between badgers and cattle that we base on the differences in prevalence. 


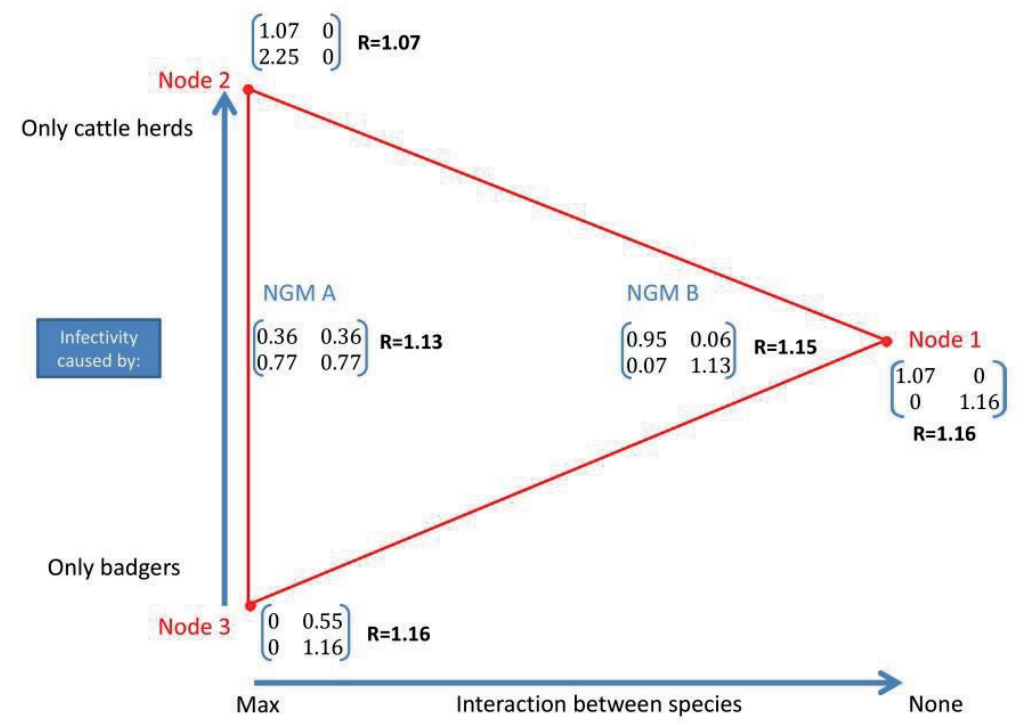

Figure 2. Range of NGMs and $R$ s for the cattle-badger system in Ireland, with and without interaction between the two species, and for a number of relative infectivities of cattle and badgers.

Summarizing, the methodology presented in this manuscript allows estimation of all plausible matrices that could describe M. bovis transmission in Ireland. We have shown the results for some of these NGMs, with a focus on transmission scenarios with very different biological interpretations. After calculating all plausible NGMs within this triangle, our results indicate that $R$ for the cattle-badger system in Ireland has to be contained within the range determined by the three extreme NGMs presented in the nodes of the triangle, i.e. $1.07<R<1.16$; indeed NGMs A and $\mathrm{B}$ show values within this interval. The reproduction ratio for the system is therefore above 1, and indicates that with current control strategies eradication of $M$. bovis from the system will not occur. These results also indicate that the maximum value the reproduction ratio for cattle to cattle transmission can take is 1.07 , above 1 but not far from it. Indeed, for most of the plausible NGMs, this value is below 1. This result is not surprising as with the current test and remove controls in cattle, the within-herd transmission is likely to be low, and this will also apply to the betweenherd transmission. The fact that $R_{c c}<1$ in most of the NGMs has large implications in terms of selecting appropriate eradication strategies, suggesting that additional intervention strategies applied to badgers (if successful), could lead to eradication.

Impact of BCG vaccination in badgers on the cattle-badger system

Following on from the results presented above, here we explore the impact of badger vaccination on the badger-cattle system. As BCG badger vaccination at $100 \%$ 
vaccine coverage can reduce $R_{b b}$ by $59 \%$ (Aznar et al., 2017), then the reproduction ratio for the system after BCG badger vaccination $\left(R_{b b(1)}\right)$ for the maximum plausible value of $R_{b b}=1.16$ is $R_{b b(1)}=(1-0.59) * 1.16=0.48$. This suggests that badger vaccination can achieve $R_{b b}<1$ in all transmission scenarios. Nonetheless, there are a small number of plausible scenarios where transmission from badgers to cattle $\left(R_{b c}\right)$ is so small, that adding control strategies in badgers (such as vaccination) would have very little impact on $R_{0}$ because in those cases $R_{c c}$ is above 1 . To delimit the area where BCG vaccination cannot achieve eradication of $M$. bovis from the system we looked at areas in the triangle where $R_{c c} \geq 1$. There are two nodes where $R_{c c} \geq 1$ (nodes 1 and 2), this is also the case for the NGMs on the line connecting both nodes (as $R_{b c}=0$ in this line and $R_{c c}=1.07$ as in nodes 1 and 2). This indicates that the area where $R_{c c} \geq 1$ is close to this line. To delimit this area further, we assessed a number of $M^{\prime}$ series to obtain the $x$ values for which $R_{c c}=1$. For that, similarly to the derivations presented in Material and Methods 3.2.2, were two elements of a matrix $M^{\prime}$ were obtained from a 2 equation system, the values of $x$ for which $R_{c c}=1$ from these four matrices series: $M^{\prime}=\left(\begin{array}{cc}E_{c c}^{\prime} & x E_{b b}^{\prime} \\ x E_{c c}^{\prime} & E_{b b}^{\prime}\end{array}\right), M^{\prime}=$ $\left(\begin{array}{cc}E_{c c}^{\prime} & x E_{c c}^{\prime} \\ x E_{b b}^{\prime} & E_{b b}^{\prime}\end{array}\right), M^{\prime}=\left(\begin{array}{cc}E_{c c}^{\prime} & x E_{b c}^{\prime} \\ E_{b c}^{\prime} & x E_{c c}^{\prime}\end{array}\right)$, and $M^{\prime}=\left(\begin{array}{cc}E_{c c}^{\prime} & x E_{c c}^{\prime} \\ E_{b c}^{\prime} & x E_{b c}^{\prime}\end{array}\right)$ were estimated. These matrices series are shown in Figure 3 as blue lines (except for the last matrix series which represents the series of matrices based on the separable mixing assumption and are shown below the red line that goes from nodes 2 to 3 ), with the NGMs at which $R_{c c}$ becomes 1 shown as black dots. An approximation of the area where $R_{c c} \geq$ 1 and eradication is not feasible is then shown in yellow and is the result of joining these black dots using straight lines. 


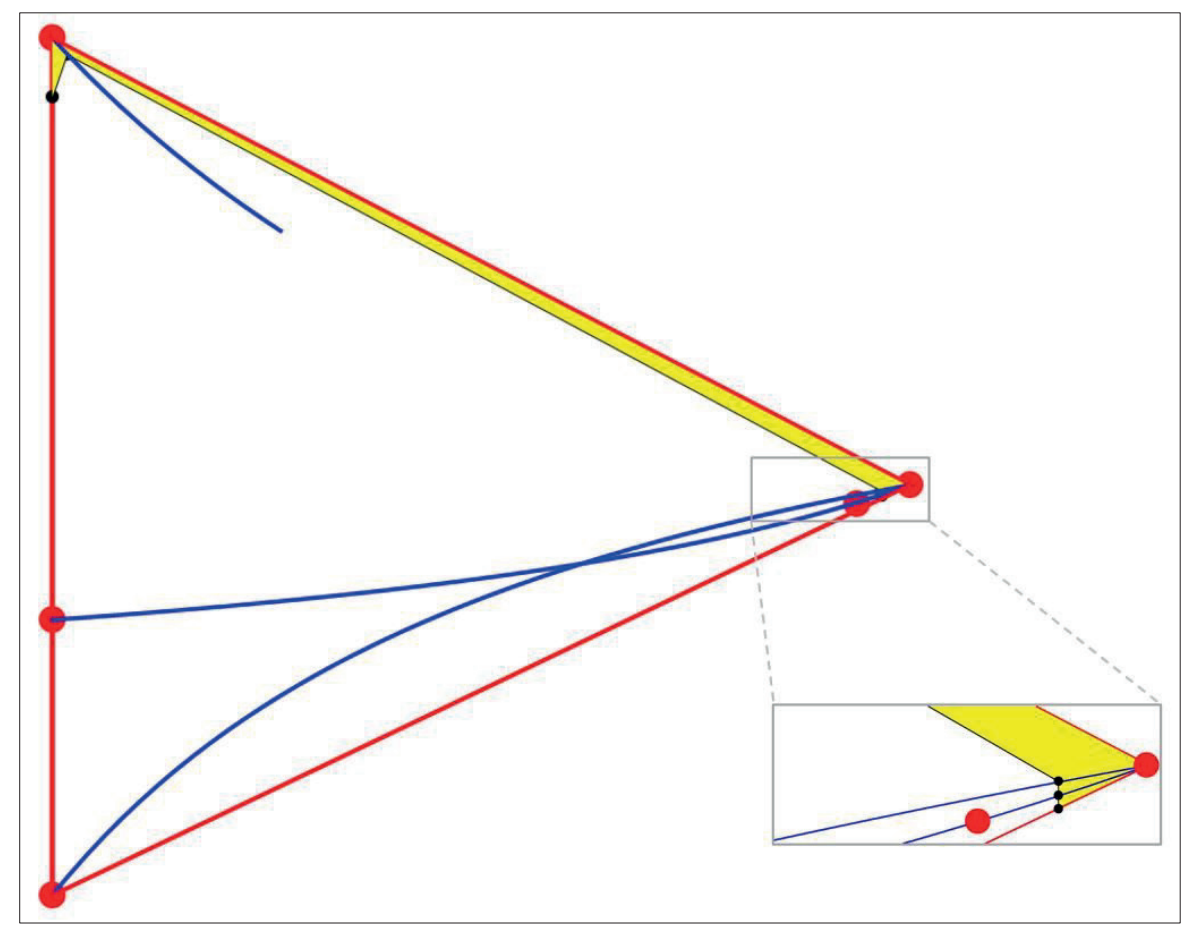

Figure 3. Transmission triangle showing the area (yellow) where eradication (by adding BCG badger vaccination to current control strategies) would not be feasible. The NGMs at the nodes of the triangle and NGMs A and B are shown as red dots. Four series of NGMs are shown as blue lines (a vertical blue line cannot be seen as coincides with the triangle boundary) with four black dots (one in each line) showing the NGMs at which $R_{c c}=1$. A magnified view of the area surrounding node 1 is also presented

This analysis helps to visualize in a simple way the magnitude of the scenarios for which eradication, by adding BCG badger vaccination (yellow area), would not be feasible. Aside, by examining the location of this area within the triangle, we can extrapolate some biological interpretations of the matrices contained in this area. All the matrices along this triangle's boundary represent a biological scenario where badgers do not transmit any infection to cattle $\left(R_{b c}=0\right)$. Similarly, the area close to this boundary, is made of scenarios where transmission from badgers to cattle is very small, with the edges indicating that either there is no transmission between cattle and badgers or all or most of it is originated from cattle. Based on literature available, we can be confident that the transmission scenarios that fall within this yellow area are not very likely to reflect field transmission between cattle and badgers in Ireland. For example, large scale experimental field trials in Ireland (O'Mairtin et al. 1998; Griffin et al. 2005) have determined that the removal of badgers is associated with 
reduced bTB risk to cattle in the removal areas compared to that in matched reference areas.

$\underline{\text { Sensitivity analysis }}$

As there was some uncertainty in terms of the total number of badgers in contact with cattle herds and the prevalence of $M$. bovis infection in this species, $R_{c c}, R_{b b}$ and $R$ (under the assumption of separable mixing and equal infectivity of badgers and cattle herds) were estimated for a range of prevalence and number of badgers values, as presented in Table $1 . R_{b b}$ and $R$ were also assessed assuming $40 \%$ and $100 \%$ vaccine coverage.

Table 1. $R_{c c}, R_{b b}$ and $R$ estimated under the assumption of separable mixing and equal infectivity of badgers and cattle herds for a range of prevalence and badger numbers, in the absence of vaccination. $R_{b b}$ and $R$ are also calculated assuming badger vaccination at $40 \%$ and $100 \%$ vaccine coverage, and are presented as $R_{b b(0.4)}$ and $R_{b b(1)}$ and $R_{0.4}$ and $R_{1}$, respectively

\begin{tabular}{|c|c|c|c|c|c|c|c|c|}
\hline \multirow[b]{2}{*}{ No. Badgers } & \multirow[b]{2}{*}{ Prev Badgers } & \multicolumn{3}{|c|}{$0 \%$ coverage } & \multicolumn{2}{|c|}{$40 \%$ coverage } & \multicolumn{2}{|c|}{$100 \%$ coverage } \\
\hline & & $\mathbf{R}_{\mathrm{cc}}$ & $\mathbf{R}_{\mathbf{b b}}$ & $\mathbf{R}$ & $\mathbf{R}_{\mathrm{bb}(0.4)}$ & $\mathrm{R}_{0.4}$ & $R_{\mathrm{bb}(1)}$ & $R_{1}$ \\
\hline 63,000 & $14 \%$ & 0.36 & 0.77 & 1.13 & 0.59 & 0.95 & 0.32 & 0.68 \\
\hline 90,000 & $14 \%$ & 0.28 & 0.85 & 1.14 & 0.65 & 0.93 & 0.35 & 0.63 \\
\hline 63,000 & $14 \%$ & 0.36 & 0.77 & 1.13 & 0.59 & 0.95 & 0.32 & 0.68 \\
\hline 40,000 & $14 \%$ & 0.48 & 0.64 & 1.12 & 0.49 & 0.97 & 0.26 & 0.74 \\
\hline 63,000 & $30 \%$ & 0.21 & 1.15 & 1.36 & 0.88 & 1.09 & 0.47 & 0.68 \\
\hline 63,000 & $14 \%$ & 0.36 & 0.77 & 1.13 & 0.59 & 0.95 & 0.32 & 0.68 \\
\hline 63,000 & $10 \%$ & 0.45 & 0.64 & 1.09 & 0.49 & 0.94 & 0.26 & 0.71 \\
\hline
\end{tabular}

In Table 1, we present the partial and system reproduction ratios when assuming separable mixing and equal infectivity of cattle and badgers while varying badger prevalence and total number of badgers for $0 \%, 40 \%$ and $100 \%$ vaccination coverage. A minimum badger prevalence of $10 \%$ and a maximum of $30 \%$ were explored; likewise we explored changes in the number of total badgers ( $\mathrm{min}=40,000$, $\max =90,000$ ). Badger vaccination with $100 \%$ coverage was able to achieve eradication of $M$. bovis from the system in all the scenarios presented. That was also the case when vaccination was used with $40 \%$ rather than $100 \%$ coverage, except for the scenario assuming $30 \%$ prevalence in badgers. Here the reproduction ratio was above but close to $1\left(R_{0.4}=1.09\right)$.

Conclusion

BCG vaccination of badgers has the potential to achieve eradication of $M$. bovis infection in the cattle-badger system in Ireland, unless there is limited transmission from badgers to cattle (implying that almost all infection in cattle originates from 
cattle). The transmission scenarios, where $R_{c c}>1$ and therefore eradication by adding BCG vaccination would not be feasible, represent a very small proportion of all possible transmission scenarios that can explain the observed data (yellow area in Figure 3); also, these scenarios would be highly unlikely to represent transmission in the field. In all other scenarios vaccination in badgers together with the current interventions in cattle lead to eradication of bTB from the system.

Achieving $100 \%$ BCG badger vaccination coverage in the field, regardless of deployment methodology, will not be possible. Nonetheless, the results obtained when vaccination coverage of $40 \%$ is assumed are still quite promising, with eradication of $M$. bovis being feasible in most scenarios.

We have arrived at these conclusions by deriving equations that allow calculation of $R$ and the NGM at the disease free equilibrium based on the observed endemic equilibrium data. The straightforwardness of the data required will make this methodology very appealing in many scenarios where multiple hosts are involved. By using this methodology we have been also able to reduce the uncertainty introduced by the lack of reliable transmission parameters between cattle and badgers.

\section{Material and Methods}

\subsection{Data}

To define the $M$. bovis cattle-badger system in Ireland, we first looked at the landscape where these two populations overlap and where interspecies transmission is feasible. In this paper, cattle to cattle transmission $\left(R_{c c}\right)$ represents the transmission of $M$. bovis between cattle belonging to different herds caused by nose to nose contact, environmental contamination, or cattle movements. To estimate the number of cattle herds in contact with the badger population, and using the Department of Agriculture, Food and the Marine (DAFM) Wildlife Unit (WU) database, a file was extracted of badger setts $(n=36,095)$ assigned for capture linked to farms that had triggered badger culling between 2004 and 2015. This file was merged with a geodatabase containing badger sett locations using ArcGIS (ESRI, ArcGIS Desktop: Release 10.3 Redlands, CA: Environmental Systems Research Institute). A buffer with a $500 \mathrm{~m}$ radius was generated around each of these setts. This distance was selected to represent the average distance from a main sett to the edge of a badger social group territory and is the distance used by DAFM in their interim badger culling program to calculate 'treated' land. Where buffers overlapped, their intersecting boundaries were dissolved. Using the DAFM Land Parcel Identification System (LPIS) for 2015, all cattle herds intersecting the 500 metre buffer area were identified $(n=72,288)$. The estimated bTB prevalence at cattle herd level for 2015 in the buffer area was $6.3 \%$. The prevalence was calculated using the Animal Health 
Computer System (AHCS) database from DAFM as the percentage of the herds in the buffer area that were restricted in 2015 with 1 or more animals identified as skin reactors or lesions as calculated by Gallagher et al. (2013).

The pre-culled badger population in Ireland was estimated at 84,000 (Sleeman et al., 2009). These badgers reside in approximately 19,000 social groups (Byrne et al. 2014) with a higher proportion of social groups occurring within higher bTB risk areas. To derive a badger population estimate for the present simulations, we assumed that $40 \%$ of the badger population was culled (based on social groups; Byrne et al. 2014) at a conservative culling efficacy of 64\% (Byrne et al. 2013; $\mathrm{n}=63,000$ ). A minimum number of 40,000 and a maximum of 90,000 badgers were also selected for the sensitivity analysis. In Byrne et al. (2015), two partial badger apparent prevalence estimates were reported: $17.7 \%$ for the period from May 2007 to May 2011, and 9.9\% for the period from June 2011 to April 2013 when PCR techniques were introduced (biochemical tests were used until May 2011).

Although an average badger prevalence of 14\% was used in the current analysis, a maximum prevalence of $30 \%$ and a minimum of $10 \%$ (based on expert opinion) were also explored. The effects of BCG vaccination with $100 \%$ and $40 \%$ coverage in $R_{b b}$ and subsequently in $R$ were estimated using estimates for vaccine efficacy for susceptibility $\mathrm{VE}_{\mathrm{S}}=59 \%$ and vaccine efficacy for infectiousness $\mathrm{VE}_{\mathrm{I}}=0 \%$, as previously described by Aznar et al. (2017).

\subsection{Derivations}

Equations to estimate $R$ and the NGM under the assumption of disjunct mixing (node 1) are described in the introduction. As no interaction is assumed between species $\left(R_{c b}=R_{b c}=0\right)$, each of the intra-species reproduction ratios $\left(R_{c c}\right.$ and $\left.R_{b b}\right)$ can be calculated as $\frac{1}{1-\text { prev }}$, with the system $R$ being the largest of either $R_{c c}$ or $R_{b b}$.

Below, we first present the derivations to estimate any plausible NGM and $R$ under the assumption of separable mixing, including the two extreme NGMs that form nodes 2 and 3 (section 3.2.1). Secondly, we show the derivations to estimate a number of series of matrices that do not necessarily assume separable mixing (but could) within the transmission triangle, including the NGMs that form the lines joining nodes 1 and 2 and nodes 1 and 3 (section 3.2.2). Although any NGM within the triangle can be estimated using the later derivations, derivations presented in section 3.2.1 are needed to confirm whether or not all NGMs under the assumption of separable mixing fall on the line between nodes 2 and 3. Note that all plausible NGMs in this triangle will have a standardized eigenvector equal to the ratio of the prevalence of $M$. bovis infection in cattle herds to that in badgers.

\subsubsection{Derivations to estimate any NGM and $R$ under the assumption of separable mixing}


Assuming separable mixing, we first show the derivations to estimate $R^{\prime}$ (reproduction ratio at the endemic equilibrium) from data observed at the endemic equilibrium (section a.). In section b., the derivations to estimate the Jacobian matrix at the endemic and at the disease free equilibrium (NGM) are shown. To be able to estimate these NGMs, it was necessary to estimate the susceptibility of cattle herds and badgers; the derivations to estimate the susceptibility of the two hosts are presented in section c.

a. Derivations to estimate $R^{\prime}$ in the cattle-badger system from the endemic equilibrium assuming separable mixing

If we assume that there is separable mixing between both species (cattle and badgers), then, the force of infection in each of the species will come from both cattle and badgers and the model equations for a SIR (Susceptible-Infectious-Recovered) model can be written as:

$$
\begin{aligned}
& \frac{d I_{c}}{d t}=\beta_{c c} \frac{I_{c} S_{c}}{N}+\beta_{b c} \frac{I_{b} S_{c}}{N}-\alpha I_{c} \\
& \frac{d I_{b}}{d t}=\beta_{c b} \frac{I_{c} S_{b}}{N}+\beta_{b b} \frac{I_{b} S_{b}}{N}-\alpha I_{b}
\end{aligned}
$$

where $\alpha$ is the recovery rate, and $\beta$ the transmission rate parameter with indices " $c$ " and " $b$ " referring to the hosts, cattle (farms) and badgers (individuals) respectively, and the direction of the transmission determined by the location of these indices, i.e. $\beta_{b c}$ is the transmission rate from badgers to cattle herds.

Under the assumption of separable mixing, we can then define the transmission rate from cattle herds to badgers or $\beta_{c b}$ as the product of the contact rate $(c)$, the susceptibility of badgers $\left(\gamma_{b}\right)$ and the infectivity of cattle $\left(\varphi_{c}\right)$, or $\beta_{c b}=c \gamma_{b} \varphi_{c}$; likewise we can define $\beta_{c c}=c \gamma_{c} \varphi_{c}, \beta_{b c}=c \gamma_{c} \varphi_{b}$ and $\beta_{b b}=c \gamma_{b} \varphi_{b}$.

By substitution, the model equations can then be written as:

$$
\begin{aligned}
& \frac{d I_{c}}{d t}=c \gamma_{c} \frac{S_{c} I_{T O T}}{N}\left(\varphi_{c} \frac{I_{c}}{I_{T O T}}+\varphi_{b} \frac{I_{b}}{I_{T O T}}\right)-\alpha I_{c}=c \gamma_{c} \overline{\varphi_{t}} \frac{S_{c} I_{T O T}}{N}-\alpha I_{c} \\
& \frac{d I_{b}}{d t}=c \gamma_{b} \frac{S_{b} I_{T O T}}{N}\left(\varphi_{c} \frac{I_{c}}{I_{T O T}}+\varphi_{b} \frac{I_{b}}{I_{T O T}}\right)-\alpha I_{b}=c \gamma_{b} \bar{\varphi}_{t} \frac{S_{b} I_{T O T}}{N}-\alpha I_{b}
\end{aligned}
$$

Where $\overline{\varphi_{t}}$ is the total average infectivity at time t.

Then, by adding both equations:

$$
\begin{aligned}
& \frac{d I_{T O T}}{d t}=c \overline{\varphi_{t}} \frac{S_{T O T} I_{T O T}}{N}\left(\gamma_{c} \frac{S_{c}}{S_{T O T}}+\gamma_{b} \frac{S_{b}}{S_{T O T}}\right)-\alpha I_{T O T}=c \bar{\gamma}_{t} \frac{\bar{\varphi}_{t}}{\frac{S_{T O T} I_{T O T}}{N}}-\alpha I_{T O T} \\
& \frac{d I_{T O T}}{d t}=\alpha I_{T O T}\left(\frac{c \overline{\gamma_{t}} \overline{\varphi_{t}}}{\alpha} \cdot \frac{S_{T O T}}{N}-1\right) \text { where } \frac{c \overline{\gamma_{t}} \overline{\varphi_{t}}}{\alpha}=R_{t}
\end{aligned}
$$

with $\overline{\gamma_{t}}$ being the total average susceptibility and $R_{t}$ being the reproduction ratio at time $t$, respectively.

As the system is at the endemic equilibrium, then $\frac{d I_{T O T}}{d t}=0$, and this is satisfied if 
$I_{T O T}^{*}=0$ (disease free equilibrium) or $R_{t} \frac{S_{T O T}^{*}}{N}-1=0$ (endemic equilibrium) and therefore:

$$
\begin{gathered}
R_{t}=\frac{N}{S_{T O T}^{*}}=\frac{1}{f_{S}^{T O T}} \text { or } \\
R^{\prime}=\frac{1}{f_{S}^{T O T}}(4)
\end{gathered}
$$

Were $f_{S}^{T O T}$ is the fraction of susceptible cattle herds and badgers out of the total population of cattle herds and badgers. Note that this $\mathrm{R}$ is based on the average susceptibility at the endemic equilibrium and this average susceptibility is different to that calculated at the disease free equilibrium.

b. Estimation of the Jacobian matrix for cattle and badgers at the endemic and the disease free equilibrium (NGM)

Here, we present how the Jacobian matrix at the endemic equilibrium for cattle herds and badgers can be calculated. Then, we demonstrate how the NGM at the disease free equilibrium and the real $R$ can be extrapolated from the endemic equilibrium matrix by taking into account the relative increase in the average susceptibility of the most susceptible type.

b1) Endemic equilibrium

Under the assumption of separable mixing, each of the components of the NGM for the cattle-badger system near the endemic equilibrium can be calculated as a function of the relative susceptibility and relative infectivity of cattle and badgers $\left(\frac{\gamma_{c}}{\overline{\gamma_{t}}}\right.$, $\left.\frac{\gamma_{b}}{\overline{\gamma_{t}}}, \frac{\varphi_{c}}{\overline{\varphi_{t}}}, \frac{\varphi_{b}}{\overline{\varphi_{t}}}\right)$, the contact rate (c), and the fractions of susceptible cattle and badgers $\left(f_{c}^{S}, f_{b}^{S}\right)$ (Diekmann et al., 2010). The Jacobian matrix in the endemic equilibrium for cattle and badgers can then be written as a $2 \times 2$ matrix with the relative infectivity of cattle and badgers presented as columns $\left(x_{c}=\frac{\varphi_{c}}{\overline{\varphi_{t}}}\right.$ and $\left.x_{b}=\frac{\varphi_{b}}{\overline{\varphi_{t}}}\right)$ and their relative susceptibility as rows $\left(y_{c}=\frac{\gamma_{c}}{\overline{\gamma_{t}}}\right.$ and $\left.y_{b}=\frac{\gamma_{b}}{\overline{\gamma_{t}}}\right)$ :

\begin{tabular}{c|c|c|}
\hline Susc & $\boldsymbol{x}_{\boldsymbol{c}}$ & $\boldsymbol{x}_{\boldsymbol{b}}$ \\
\hline $\boldsymbol{f}_{c}^{\boldsymbol{S} \boldsymbol{y}_{\boldsymbol{c}}}$ & $c x_{c} y_{c} f_{c}^{S}$ & $c x_{b} y_{c} f_{c}^{S}$ \\
\hline $\boldsymbol{f}_{\boldsymbol{b}}^{S} \boldsymbol{y}_{\boldsymbol{b}}$ & $c x_{c} y_{b} f_{b}^{S}$ & $c x_{b} y_{b} f_{b}^{S}$ \\
\hline
\end{tabular}


with $R_{c c}^{\prime}=c x_{c} y_{c} f_{c}^{S}$ and $R_{b b}^{\prime}=c x_{b} y_{b} f_{b}^{S}$ being the partial reproduction ratios at the endemic equilibrium for cattle herd to cattle herd transmission and badger to badger transmission, respectively.

At the endemic equilibrium, the fractions of infected cattle herds and infected badgers are connected to the fraction of cattle and badgers among the susceptibles as: $\left(\begin{array}{l}f_{c}^{I} \\ f_{b}^{I}\end{array}\right)=\left(\begin{array}{l}y_{c} f_{c}^{S} \\ y_{b} f_{b}^{S}\end{array}\right)$, and the addition of these two fractions is equal to 1 . Therefore if we take $y_{c} f_{c}^{S}=a$, it follows that $y_{b} f_{b}^{S}=1-a$.

As the contact rate is equal to $c=\frac{1}{f_{S}^{T O T}}$, by substitution in the NGM above, we can derive that:

$$
\begin{gathered}
R_{c c}^{\prime}=\frac{1}{f_{S}^{T O T}} x_{c} a \\
R_{b b}^{\prime}=\frac{1}{f_{S}^{T O T}} x_{b}(1-a)
\end{gathered}
$$

If we assume separable mixing, then we can calculate the reproduction ratio of the system at the endemic equilibrium as: $R_{t}=R^{\prime}=R_{c c}^{\prime}+R_{b b}^{\prime}$ and rearranging the equations above:

$$
R_{t}=\frac{1}{f_{S}^{T O T}}\left(a x_{c}+(1-a) x_{b}\right)
$$

As we showed in section a) that $R_{t}=\frac{1}{f_{S}^{T O T}}$ then

$$
\left(a x_{c}+(1-a) x_{b}\right)=1
$$

Hence, $x_{b}=\frac{1}{(1-a)}-\frac{a}{(1-a)} x_{c}$ with one possible solution being that the relative infectivity of cattle herds is $x_{c}=1$ and the relative infectivity of badgers $x_{b}=\frac{(1-a)}{(1-a)}=$ 1.

For $x_{b}=1$ and $x_{c}=1$ then

$$
\begin{gathered}
R_{b b}^{\prime}=\frac{1}{f_{S}^{T O T}}(1-a)(5) \\
R_{c c}^{\prime}=\frac{a}{f_{S}^{T O T}}(6)
\end{gathered}
$$

If we assume that all the infectivity comes from cattle, and badgers do not play any

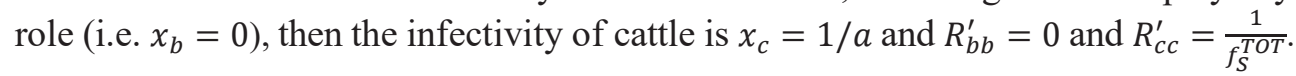
If, we assume that all the infectivity comes from badgers (i.e. $x_{c}=0$ ), then $x_{b}=\frac{1}{(1-a)}$ and $R_{b b}^{\prime}=\frac{1}{f_{S}^{T O T}}$ and $R_{c c}^{\prime}=0$.

Note that these are solutions for the partial reproduction ratios at the endemic equilibrium but to estimate the system NGM we need to estimate the partial reproduction ratios at the disease free equilibrium.

\section{b2) Disease free equilibrium}


We can now extrapolate the NGM at the disease free equilibrium from the matrix at the endemic equilibrium presented in section b1). Here, and for demonstration purposes, we first assume that heterogeneity between cattle and badgers is exclusively due to differences in their susceptibility, i.e. the infectivity of both species being equal.

If susceptibility differs between species, we can define a new contact rate $c^{\prime}=$ $\frac{1}{f_{S}^{T O T}} \frac{\overline{\gamma_{0}}}{\overline{\gamma_{t}}}$, where $\frac{\overline{\gamma_{0}}}{\overline{\gamma_{t}}}$ reflects the relative increase in the average susceptibility of the most susceptible species at the endemic equilibrium rather than at the disease free equilibrium ( $\overline{\gamma_{0}}$ represents the average susceptibility at the disease free equilibrium). At the disease free equilibrium, all individuals, cattle and badgers, are susceptible so their relative susceptibility can be expressed as $y_{c}^{\prime}=\frac{\gamma_{c}}{\overline{\gamma_{0}}}$ and $y_{b}^{\prime}=\frac{\gamma_{b}}{\overline{\gamma_{0}}}$; likewise the fractions of susceptible cattle and susceptible badgers can be written as $f_{c}^{T O T}$ and $f_{b}^{T O T}$ respectively.

The NGM at the disease free equilibrium is presented below:

\begin{tabular}{c|c|c|}
\hline Susc & $\boldsymbol{x}_{c}^{\prime}$ & $\boldsymbol{x}_{\boldsymbol{b}}^{\prime}$ \\
\hline $\boldsymbol{f}_{c}^{\text {tot } \boldsymbol{y}_{\boldsymbol{c}}^{\prime}}$ & $c^{\prime} x_{c}^{\prime} y_{c}^{\prime} f_{c}^{T O T}$ & $c^{\prime} x_{b}^{\prime} y_{c}^{\prime} f_{c}^{T O T}$ \\
\hline $\boldsymbol{f}_{b}^{\text {tot } y_{\boldsymbol{b}}^{\prime}}$ & $c^{\prime} x_{c}^{\prime} y_{b}^{\prime} f_{b}^{T O T}$ & $c^{\prime} x_{b}^{\prime} y_{b}^{\prime} f_{b}^{T O T}$ \\
\hline
\end{tabular}

As we did previously in section b., we can now make $y_{c}^{\prime} f_{c}^{T O T}=a^{\prime}$ and $y_{b}^{\prime} f_{b}^{T O T}=(1-$ $a^{\prime}$ ). By substituting in $R_{c c}$ and $R_{b b}$ (reproduction ratios for cattle to cattle transmission and badger to badger transmission at the disease free equilibrium):

with

$$
\begin{gathered}
R_{c c}=c^{\prime} x_{c}^{\prime} a^{\prime}(7) \\
R_{b b}=c^{\prime} x_{b}^{\prime}\left(1-a^{\prime}\right)(8)
\end{gathered}
$$

$$
R=c^{\prime} x_{c}^{\prime} a^{\prime}+c^{\prime} x_{b}^{\prime}\left(1-a^{\prime}\right)(9)
$$

As cattle herds and badgers have the same infectivity $\left(x^{\prime}{ }_{c}=x^{\prime}{ }_{b}=1\right)$, then:

$$
R=c^{\prime}\left(a^{\prime}+\left(1-a^{\prime}\right)\right)=c^{\prime}=\frac{1}{f_{S}^{T O T}} \frac{\overline{\gamma_{0}}}{\overline{\gamma_{t}}}
$$

Similar to the calculations done at the endemic equilibrium, we can also calculate $R$ and the two extreme NGMs that form nodes 2 and 3 in Figure 1, by assuming all 
infectivity is coming from cattle (the infectivity of badgers is null $\left(x_{b}^{\prime}=0\right)$ ), or all infectivity is coming from badgers $\left(x_{c}^{\prime}=0\right)$ respectively.

If we assume that badgers do not play any role in transmission $\left(x_{b}^{\prime}=0\right)$, then from the endemic equilibrium derivations $x_{c}^{\prime}=\frac{1}{a}$ and:

with $R_{c b}=\frac{\frac{\gamma_{c}}{\gamma_{t}}}{\frac{\gamma_{b}}{\gamma_{t}}} R_{c c}$

$$
R=R_{c c}=c^{\prime} a^{\prime} \frac{1}{a}=c^{\prime} \frac{a^{\prime}}{a}=\frac{f_{c}^{T O T}}{f_{S}^{T O T} f_{c}^{S}}=\frac{1}{1-P R E V_{c}}
$$

The resultant NGM will correspond to node 2 in the transmission triangle in Figure 1.

If transmission only originates from badgers, then from the endemic equilibrium derivations we can see that $x_{b}^{\prime}=\frac{1}{1-a}$ and:

$$
\begin{gathered}
R=R_{b b}=c^{\prime} x_{b}^{\prime}\left(1-a^{\prime}\right)=\frac{1}{f_{S}^{T O T}} \frac{\overline{\gamma_{0}}}{\overline{\gamma_{t}}} \frac{\left(1-a^{\prime}\right)}{1-a}=\frac{1}{f_{S}^{T O T}} \frac{\overline{\gamma_{0}}}{\overline{\gamma_{t}}} \frac{y_{b}^{\prime} f_{b}^{T O T}}{y_{b} f_{b}^{S}} \\
=\frac{1}{f_{S}^{T O T}} \frac{\overline{\gamma_{0}}}{\overline{\gamma_{t}}} \frac{\frac{\overline{\gamma_{b}}}{\overline{\gamma_{0}}} f_{b}^{T O T}}{\frac{\gamma_{b}}{\overline{\gamma_{t}}} f_{b}^{S}} \\
R=R_{b b}=\frac{f_{b}^{T O T}}{f_{S}^{T O T} f_{b}^{S}}=\frac{1}{1-P R E V_{b}}
\end{gathered}
$$

and $R_{b c}=\frac{\frac{\gamma_{b}}{\gamma_{t}}}{\frac{\gamma_{c}}{\gamma_{t}}} R_{b b}$

$P R E V_{b}$ and $P R E V_{c}$ represent the prevalence of $M$. bovis infection in badgers and cattle herds, respectively. The resultant NGM will be node 3 in the transmission triangle (Figure 1).

c. Derivations to estimate the relative susceptibility of cattle and badgers

If we assume a SIR model under the endemic equilibrium, the differential equation that describes the fraction of infected cattle $\left(f_{c}^{I}\right)$ can be written as:

because at the endemic equilibrium $\frac{d I_{T O T}}{d t}=0$.

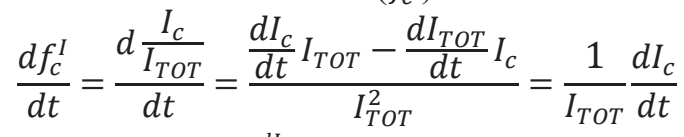

If we substitute $\frac{d I_{c}}{d t}$ for equation (1), shown in 3.2.1 section a., then:

$$
\frac{d f_{c}^{I}}{d t}=c \gamma_{c} \overline{\varphi_{t}} \frac{S_{c}}{N}-\alpha \frac{I_{c}}{I_{T O T}}=c \gamma_{c} \overline{\varphi_{t}} \frac{S_{c}}{N}-\alpha f_{c}^{I}
$$

As the fraction of infected cattle remains constant in the endemic equilibrium $\left(\frac{d f_{c}^{I}}{d t}=\right.$ $0)$, then 


$$
\alpha f_{c}^{I}=c \gamma_{c} \overline{\varphi_{t}} \frac{S_{c}}{N}
$$

Similarly, we can write the differential equation that describes the fraction of infected badgers $\left(f_{b}^{I}\right)$ and substituting $\frac{d I_{b}}{d t}$ for equation (2):

Then

$$
\alpha f_{b}^{I}=c \gamma_{b} \overline{\varphi_{t}} \frac{S_{b}}{N}
$$

And as $f_{c}^{I}+f_{b}^{I}=1$ :

$$
\frac{f_{c}^{I}}{f_{b}^{I}}=\frac{\gamma_{c} S_{c}}{\gamma_{b} S_{b}}
$$

$$
\begin{gathered}
\frac{f_{c}^{I}}{1-f_{c}^{I}}=\frac{\gamma_{c} S_{c}}{\gamma_{b} S_{b}} \\
f_{c}^{I}=\frac{\gamma_{c} S_{c}}{\gamma_{c} S_{c}+\gamma_{b} S_{b}}=\frac{\gamma_{c} f_{c}^{S}}{\gamma_{c} f_{c}^{S}+\gamma_{b} f_{b}^{S}}=\frac{\gamma_{c}}{\overline{\gamma_{t}}} f_{c}^{S}
\end{gathered}
$$

Therefore the susceptibility of cattle can be calculated as:

$$
\frac{\gamma_{c}}{\overline{\gamma_{t}}}=\frac{f_{c}^{I}}{f_{c}^{s}}
$$

Following a similar derivation for badgers, the susceptibility of badgers can be written as:

$$
\frac{\gamma_{b}}{\overline{\gamma_{t}}}=\frac{f_{b}^{I}}{f_{b}^{S}}
$$

with the relative susceptibility of cattle herds compared to badgers resulting from dividing these two fractions. As all the possible solutions for NGMs assuming separable mixing share susceptibility estimates, these derivations indicate that all these NGMs will fall on the same line of the plane. Thus, showing that indeed the NGMs triangle can be closed.

\subsubsection{Estimation of any $N G M$ and $R$ that is part of the triangle}

All Jacobian matrices that follow from the prevalence and number of cattle herds and badgers observed in Ireland at the endemic equilibrium share the same eigenvector $e$, including the three extreme $M^{\prime}$ s from which the NGMs that form the nodes of the triangle presented in Figure 1 are calculated. For all $M{ }^{\prime} \mathrm{s}$ :

For all these matrices also: $M^{\prime} * e=e$

$$
e=\left(\begin{array}{c}
e_{c} \\
e_{b}
\end{array}\right)=\left(\begin{array}{c}
\frac{I_{c}}{I_{T O T}} \\
\frac{I_{b}}{I_{T O T}}
\end{array}\right)
$$

To solve the matrices that form the nodes of the triangle, and as in these matrices two elements are equal to 0 , we can express the relationship above as a system of two equations with two unknowns. Using as an example the $M^{\prime}$ that defines node 1 in Figure 1, $E_{c c}^{\prime}$ and $E_{b b}^{\prime}$ would be the unknowns and: 
and the two resultant equations would be:

$$
\left(\begin{array}{cc}
E_{c c}^{\prime} & 0 \\
0 & E_{b b}^{\prime}
\end{array}\right) *\left(\begin{array}{c}
\frac{I_{c}}{I_{T O T}} \\
\frac{I_{b}}{I_{T O T}}
\end{array}\right)=\left(\begin{array}{c}
\frac{I_{c}}{I_{T O T}} \\
\frac{I_{b}}{I_{T O T}}
\end{array}\right),
$$

$$
\begin{aligned}
& E_{c c}^{\prime} * \frac{I_{c}}{I_{T O T}}=\frac{I_{c}}{I_{T O T}} \\
& E_{b b}^{\prime} * \frac{I_{b}}{I_{T O T}}=\frac{I_{b}}{I_{T O T}}
\end{aligned}
$$

By solving the equations, then $E_{c c}^{\prime}=E_{b b}^{\prime}=1$ and $M^{\prime}=\left(\begin{array}{ll}1 & 0 \\ 0 & 1\end{array}\right)$.

To calculate the NGM $(M)$ for this specific matrix, then:

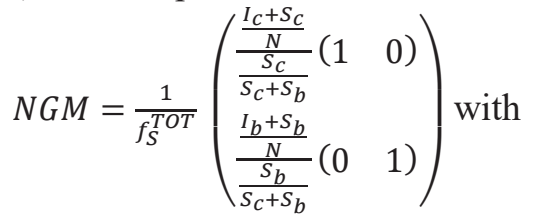

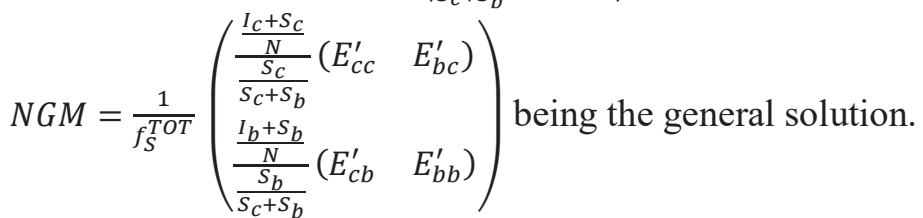

Other NGMs within the triangle can also be estimated as above by fixing one of the elements at a time of a matrix type $M^{\prime}=\left(\begin{array}{ll}E_{c c}^{\prime} & E_{b c}^{\prime} \\ E_{c b}^{\prime} & E_{b b}^{\prime}\end{array}\right)$ and estimating the solution for small increments of a second element. This will result in a number of series of matrices that starting from each of the nodes of the triangle might or might not reach one of the triangle boundaries.

\section{References}

1. Aznar, I., Frankena, K., More, S.J., O’Keeffe, J., McGrath, G., de Jong, M.C.M. 2017. Quantification of Mycobacterium bovis transmission in a badger vaccine field trial. Prev. Vet. Med. https://doi.org/10.1016/j.prevetmed.2017.10.010

2. Biemans, F., de Jong, M.C.M., Bijma, P., 2017. A model to estimate effects of SNPs on host susceptibility and infectivity for an endemic infectious disease. Genet. Sel. Evol. 30, 49(1):53. doi: 10.1186/s12711-017-0327-0.

3. Biek R., O'Hare A., Wright D., Mallon T., McCormick C., Orton R.J., McDowell S., Trewby H., Skuce R.A. and Kao R.R., 2012. Whole genome sequencing reveals local transmission patterns of Mycobacterium bovis in sympatric cattle and badger populations. PLoS Pathog. 8, e1003008.

4. Brooks-Pollock, E. and Wood, J.L.N., 2015. Eliminating bovine tuberculosis in cattle and badgers: insight from a dynamic model. Proc. R. Soc, Lond. B Biol. Sci. 282, 20150374. 
5. Brooks-Pollock, E. and Danon, L., 2017. Defining the population attributable fraction for infectious diseases. Int. J. Epidemiol. 46, 976-982.

6. Byrne, A.W., O'Keeffe, J., Sleeman, D.P., Davenport, J. and Martin, S.W., 2013. Impact of culling on relative abundance of the European badger (Meles meles) in Ireland. European journal of wildlife research, 59(1), 25-37.

7. Byrne, A.W., Acevedo, P., Green, S. and O'Keeffe, J., 2014. Estimating badger social-group abundance in the Republic of Ireland using cross-validated species distribution modelling. Ecological Indicators, 43, 94-102.

8. Byrne, A.W., Kenny, K., Fogarty, U., O’Keeffe, J.J., More, S.J., McGrath, G., Teeling, M., Martin, S.W. and Dohoo, I.R., 2015. Spatial and temporal analyses of metrics of tuberculosis infection in badgers (Meles meles) from the Republic of Ireland: Trends in apparent prevalence. Preventive veterinary medicine, 122(3), 345-354.

9. Diekmann O., Heesterbeek J.A.P. and Metz J.A.J., 1990. On the definition and computation of the basic reproduction ratio $R_{0}$ in models for infectious diseases in heterogeneous populations. J. Math. Biol. 28, 365-382.

10.Diekmann O., Heesterbeek J.A.P. and Roberts M.G., 2010. The construction of next generation matrices for compartmental epidemic models. J. R. Soc. Interface. 7, 873-885.

11.FSAI. (2017). FSAI. Retrieved 21 September, 2017, from https://www.fsai.ie/enforcement_audit/monitoring/food_surveillance/zoonoses/ reports/tuberculosis.html

12.Fulford G.R., Roberts M.G., Heesterbeek J.A.P., 2002. The metapopulation dynamics of an infectious disease: tuberculosis in possums. Theor. Popul. Biol. 61, 15-29.

13.Gallagher M.J., Higgins I.M., Clegg T.A., Williams D.H., More S.J., 2013. Comparison of bovine tuberculosis rec.urrence in Irish herds between 1998 and 2008. Prev. Vet. Med. 111, 237-244.

14.Godfray, H.C.J., Donnelly, C.A., Kao, R.R., Macdonald, D.W., McDonald, R.A., Petrokofsky, G., Wood, J.L.N., Woodroffe, R., Young, D.B., McLean, AR., 2013. A restatement of the natural science evidence base relevant to the control of bovine tuberculosis in Great Britain. Proc. R. Soc. B. 280, 20131634. http://dx.doi.org/10.1098/rspb.2013.1634

15.Good M., 2006. Bovine tuberculosis eradication in Ireland. Ir. Vet. J. 59 (3), 154162.

16.Gormley, E. and Corner, L. A. L., 2013. Control Strategies for Wildlife Tuberculosis in Ireland. Transbound Emerg Dis, 60: 128-135. doi:10.1111/tbed.12095

17.Griffin, J.M., Williams, D.H., Kelly, G.E., Clegg, T.A., O’boyle, I., Collins, J.D. and More, S.J., 2005. The impact of badger removal on the control of tuberculosis in cattle herds in Ireland. Prev. Vet. Med. 67(4), 237-266. 
18.Haydon, D.T., 2002. Identifying Reservoirs of Infection: A Conceptual and Practical Challenge. Emerg. Infect. Diseases 8(12), 1468-1473. http://doi.org/10.3201/eid0812.010317

19.Heesterbeek J.A.P.and Roberts M.G., 1995. Threshold quantities for infectious diseases in periodic environments. J. Biol. Syst. 3(3), 779-787.

20.Heesterbeek J.A.P., 2000. Mathematical epidemiology of infectious diseases: model building, analysis and interpretation. Vol. 5. John Wiley \& Sons.

21.Máirtín, D.Ó., Williams, D.H., Griffin, J.M., Dolan, L.A. and Eves, J.A., 1998. The effect of a badger removal programme on the incidence of tuberculosis in an Irish cattle population. Preventive veterinary medicine, 34(1), pp.47-56.

22.More S.J. and Good M., 2006. The tuberculosis eradication programme in Ireland: a review of scientific and policy advances since 1988. Vet. Microbiol. $112,239-251$.

23.Keeling, M., and P. Rohani, 2008. Modeling Infectious Diseases in Humans and Animals (Princeton University Press, Princeton).

24.Kermack W.O. and McKendrick A.G., 1927. A contribution to the mathematical theory of epidemics. Proc. Math. Phys. Eng. Sci. 115(772), 700-721.

25.Sleeman, D.P., Davenport, J., More, S.J., Clegg, T.A., Collins, J.D., Martin, S.W., Williams, D.H., Griffin, J.M. and O'Boyle, I., 2009. How many Eurasian badgers Meles meles L. are there in the Republic of Ireland?. European Journal of Wildlife Research, 55(4), pp.333-344. 

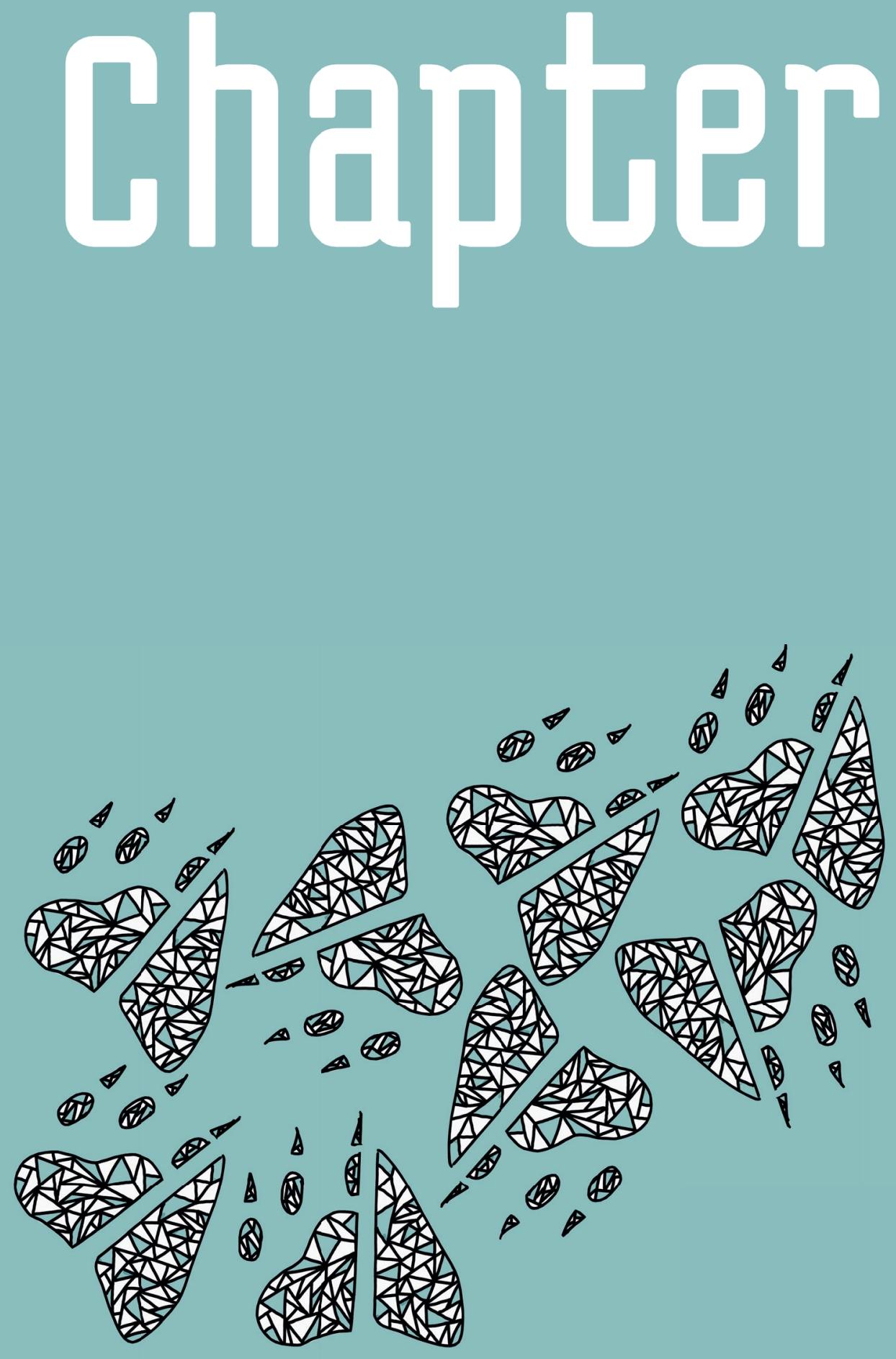


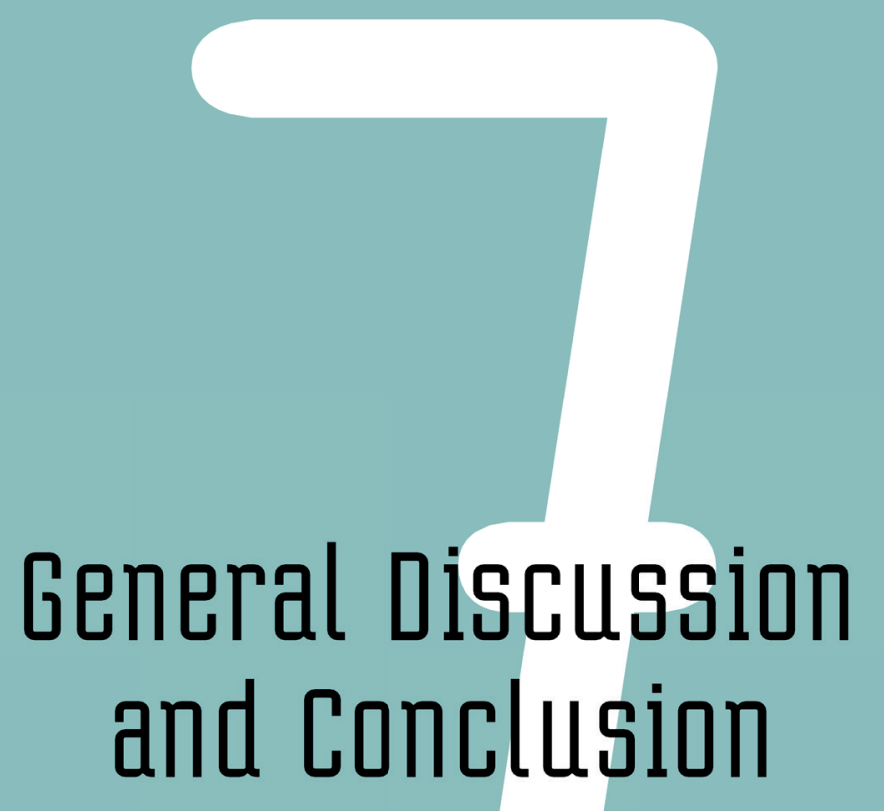




\section{Introduction}

Ireland commenced a control/eradication programme for Mycobacterium bovis infection in cattle in 1954 (More and Good, 2006). Prevalence of infection was very high, with about $80 \%$ of cattle herds being infected. Some success was achieved over the first 10 years of the control programme (animal incidence was reduced from $17 \%$ to $0.5 \%$ by 1965 ), suggesting that eradication of the infection in cattle was feasible. However, following this early success, and despite an overtime increase in cattle controls, incidence in cattle remained relatively stable (Figure 1 Chapter 1). Meanwhile, badgers (Meles meles) were confirmed as a reservoir of the infection and were suspected as the main reason for the failure to eradicate the infection in cattle (Costello et al., 1999; Griffin et al., 2005). As a result, starting in Ireland in the early 1990s, cattle farms with severe bTB outbreaks were subjected to an epidemiological investigation. Where all other possible sources of infection were ruled out and badgers were implicated, targeted removals of badgers were carried out around these farms (Olea-Popelka et al., 2009). These badger removal operations were gradually intensified and became more systematic with about 4,700 badger removal licences being issued in 2000 (O'Boyle, I., 2000).

Within this context, the main objective of this thesis was to quantitatively assess the contribution of each of the species, cattle and badgers, to infection maintenance. It also sought to answer the specific question of whether or not BCG badger vaccination should be added to the current control strategies to help in the eradication of bTB in cattle. Ultimately, the main goal was to gain enough scientific knowledge so that new policy on bovine tuberculosis (bTB), leading to eradication, could be formulated. Chapters 2 to 5 were strategically designed to assess the impact of BCG vaccination on $M$. bovis transmission in the badger population. The biological ways in which BCG vaccination worked to achieve a reduction on the M. bovis transmission in a badger population were also disentangled. In Chapter 6, by estimating all the plausible Next Generation Matrices (NGMs) for the cattle-badger system in Ireland, we were able to assess not only the effectiveness of vaccination in the badger population but also in the cattle-badger system.

Based on the results from this thesis as well as on other parallel research (Gormley et al., 2017), the Irish government signalled the intention to include BCG badger vaccination in the eradication programme for $M$. bovis in Ireland (DAFM, 2017). In this final chapter, the main findings from this thesis are discussed. The two main sets of results, one related to the confirmation of bTB working hypothesis and another to the potential of BCG vaccination to achieve $M$. bovis eradication, both of them critical to the future of the bTB strategy policy are examined. Also, a number of questions in relation to adding badger vaccination to the eradication programme that could arise from policymakers and other stakeholders are pre-empted and answered. 
A section on the challenges posed by the introduction of BCG vaccination and how these challenges can be overcome follows. Finally, suggestions for further scientific work are made.

\section{Main results}

\section{a) Confirmation of working hypotheses}

In this thesis, two working hypotheses fundamental to the design of a path to bTB eradication have been confirmed. Firstly, it has been confirmed that eradication of $M$. bovis infection in the cattle-badger system would not be feasible without the addition of new control strategies, as shown by the basic reproduction ratio estimated under current controls $\left(1.07 \leq R_{0} \leq 1.16\right)$ (Chapter 6). Secondly, it was shown that the reproduction ratio for cattle to cattle transmission was below $1\left(R_{c c}<1\right)$ for almost all plausible transmission scenarios, indicating that eradication of the infection in the system can be achieved by the addition of interventions in badgers alone. Although these are some of the general conclusions from this thesis, it is important that we further analyse these outcomes so that policy makers have a full understanding of their significance and limitations.

In relation to the first statement that current controls will not result in eradication, an important disclaimer has to be made. In our analysis. we made an inherent assumption that the cattle-badger system is at the endemic equilibrium. In this way, data observed in the field can then be extrapolated to obtain $R_{0}$ at the disease free equilibrium (where all the individuals are susceptible). In Figure 1 of the thesis introduction, it was shown that a slight decrease in bTB incidence in cattle had been observed since the introduction of badger culling. Based on that, it is possible then that the system is not at the endemic equilibrium and that if badger culling were to be continued at a similar rate, the infection could die out in the system (assuming badger culling is responsible for the decrease in incidence). In this way, the statement that eradication would not occur with current strategies would be incorrect. Nonetheless, if the observed reduction in cattle bTB incidence is real (rather than an artefact) and if this reduction is truly due to the introduction of badger removal, the statement could still be relevant as badger culling is anticipated not to be continued at the current rate (6,000 badger/year) (O'Keeffe, DAFM, personal communication).

Secondly, we have confirmed that in the majority of the plausible transmission scenarios, badgers (as opposed to cattle herds) contribute the most to the overall transmission (based on the observed prevalence of M. bovis infection in both species and the number of cattle herds and badgers). For example, assuming separable mixing and equal relative infectivity of the two species, the partial reproduction ratios, $R_{c c}$ and $R_{b b}$, were estimated, confirming the hypothesis that the contribution from badgers to $R_{0}$ is higher than that from cattle herds $\left(R_{b b}=0.77, R_{c c}=0.36\right)$. The 
significance of this statement, is that it confirms the working hypothesis that a greater leverage (in terms of impact on $R_{0}$ ) can be achieved if additional control options were to be focussed on the species that contributes the most to $R_{0}$, in this case badgers (assuming equal effectiveness, feasibility and availability of control options in the two species and assuming that resources are scarce and their optimization is imperative). Nonetheless, the statements above should not be interpreted lightly as in no case should our conclusions preclude the addition of new control strategies in cattle. Quite the opposite, not only current controls in cattle will have to be maintained for our results to be true, but adding additional or more stringent control strategies in cattle might be necessary to compensate for the fact that culling in badgers will not be maintained at the current rate. Aside, these added controls can help accelerating the rate of decrease in $R_{0}$ and therefore time to eradication. Further details about time to eradication will be discussed later in this chapter.

b) BCG vaccination in badgers has the potential to achieve eradication of $M$. bovis infection in the cattle-badger system in Ireland

In Chapter 5, BCG vaccine efficacy in badgers was estimated as $\mathrm{VE}_{\mathrm{S}}=59 \%$ and $\mathrm{VE}_{\mathrm{I}}=0 \%$. Here, it was shown how vaccination if added to current controls, was able to achieve eradication of the infection in the badger population. Nonetheless, it was in Chapter 6 where the possibility of achieving eradication of the infection from the system (in the majority of the plausible transmission scenarios) was confirmed. A biological and mathematical description of the small number of transmission scenarios where eradication would not be feasible is also presented. By looking at extreme scenarios in terms of $M$. bovis transmission in the system, all matrices that could describe transmission in cattle and badgers in Ireland were framed within a NGM triangle (with the vertices of this triangle representing the most extreme transmission scenarios) (Figures 1 and 2 in Chapter 6). Further, the approximate area within this triangle where eradication of the infection in the system (by adding BCG badger vaccination at $100 \%$ coverage) was not feasible was estimated (Figure 3 Chapter 6). This area is shown in yellow and lies parallel to the line that joins nodes 1 and 2 in the NGMs transmission triangle. Nodes 1 and 2 represent transmission scenarios where either: 1) no interaction between the two species is present (node 1), or 2) there is maximal interaction between species with all the infection originated from cattle (node 2). In both scenarios $R_{c c}=1.07$ and both would result in a reproduction ratio after adding badger vaccination (at $100 \%$ coverage) to current controls above $1\left(R_{1}=1.07\right)$. Further, the area where eradication is not possible is characterized by $R_{c c}>1$ and $R_{1}>1$. In this area, transmission from badgers to cattle is so small (or non-existent), that any control measures focused on badger transmission exclusively will not have a sufficient effect on the reproduction ratio of the system to bring it below 1. Nonetheless, as it can be observed in Figure 3, the number of transmission scenarios where $R_{c c}>1$ and therefore $R_{1}>1$ is very small. 
This leads to the conclusion that eradication by adding badger vaccination would be feasible in a large proportion of all the plausible transmission scenarios.

Very few parameters were needed to estimate the plausible NGMs that could describe the transmission between badgers and cattle herds: number of cattle herds and badgers and prevalence in both species. As cattle data are quite reliable, the sensitivity analysis focused on badger numbers and prevalence. In all the scenarios explored $R_{1}<1$ (more precisely, $R_{1}<0.74$ ). $R_{0}$ was also calculated when only $40 \%$ of the badger population was vaccinated and assuming larger /lower number of badgers and their prevalence than those used for the main analysis. The $40 \%$ vaccination coverage selected aimed to reflect badger trapping capabilities (as observed in previous vaccine trials) (Byrne et al., 2012). At this coverage, eradication was also feasible except when the badger prevalence would have exceeded $30 \%\left(R_{0.4}=1.09\right)$. Therefore in this thesis and as a difference from other modelling exercises, the uncertainty in the success of adding vaccination to the current control strategies does not depend on inaccurate parameterization but rather on the logistics of achieving sufficient vaccination coverage.

\section{c) Further insights}

One difficulty when translating the results from this thesis into policy arises from the fact that our calculations assume that current controls in cattle and badgers are going to remain in place; because badger culling rates will most likely not be maintained (O'Keeffe, DAFM, personal communication), this assumption does not stand. If we assume that the observed recent reduction in cattle incidence is due to badger culling, then, the key question is: how long will the observed NGM remain stable subsequent to reducing/abolishing badger culling? Badger culling can potentially affect transmission to cattle in two ways: by reducing badger density, and by reducing the prevalence in badgers (if culling of badgers is focused on infected badgers), both resulting in a reduction of transmission opportunities (Byrne et al., 2015). Nonetheless, there is no research yet available on the time that it would take for a badger population to recover in numbers subsequent to targeted culling, or the time that it would take for infection prevalence to raise. As a result, our recommendation would be that badger vaccination should be carried out in a manner that assures the minimum vaccination coverage is achieved before badger densities can recover. Also, and as mentioned before, this should be seen as another argument supporting that stringent controls in the cattle population are put in place along with the addition of $B C G$ vaccination.

Until now, the use of BCG vaccination in cattle has not been possible as there are no vaccine-compatible diagnostic assays capable of distinguishing vaccinated from infected cattle (Vordermeier 2016). Some research is being carried out in this field with expectations that a test with these specifications will be available in the near future. This issue has been thoroughly considered in an EFSA report (EFSA, 2013). 
Nonetheless, and apart from all regulatory and trial design issues highlighted in this report, the main question to be answered is whether or not a strategy based on testing of cattle followed by either vaccination or removal (TVR) can achieve an equal/higher impact on $R_{c c}$ (and subsequently on $R_{0}$ ) than that obtained with the removal of SICCT positive cattle. If the impact of TVR is higher than the impact from current controls in cattle, then a question that policy makers could pose is whether or not vaccinating cattle would be a better option than vaccinating badgers. Assuming that the vaccine efficacy of BCG in cattle would be similar to that observed in badgers $\left(\mathrm{VE}_{S}=59 \%\right.$ and $\left.\mathrm{VE}_{\mathrm{I}}=0 \%\right)$ and based on the NGM assuming equal infectivity of cattle herds and badgers (NGM A in Chapter 6, i.e. $R_{b b}=R_{b c}=$ 0.77 and $R_{c c}=R_{c b}=0.36$ ), a larger reduction in $R_{0}$ would be achieved by vaccinating only badgers $\left(R_{1}=0.68\right)$ compared to vaccinating only cattle $\left(R_{1}=\right.$ 0.92 ) in Ireland (in each case, assuming $100 \%$ coverage). Nonetheless, the comparison should take into account the fact that the effort/logistics and economics of vaccinating badgers are much larger than those required for vaccinating cattle. It is estimated that vaccinating $40 \%$ of the badgers would achieve a similar reduction of $R_{0}$ compared to vaccinating the entire cattle population $\left(R_{0.4}=0.95\right)$ (further calculations based on results from Chapter 6). The question that could follow is whether it would be a better strategy to wait until BCG cattle vaccination becomes available? There are still too many uncertainties on whether a TVR strategy in cattle will work/be available. As badger culling is going to be gradually reduced, awaiting for certainty in relation to this strategy (without adding badger vaccination) will result in changes to the NGM that could compromise the ability to eradicate the infection in cattle.

Another question that may arise from the results of this thesis is how long it will take to achieve eradication. There is a simple formula that can give us an approximate estimate of time to eradication based on $R_{0}$ :

$$
\text { Time }=\ln \left(\frac{1}{10}\right) / \ln \left(R_{0}\right)
$$

In the sensitivity analysis carried out in Chapter 6 (Table 1 ), it is shown how $R_{0}$ is close to 1 in all but one of the scenarios (when vaccination coverage is equal to $40 \%$ ); in most scenarios $0.9<R_{0.4}<1.0$. Using the formula above, the time to eradication for a value of $R_{0}=0.97$ was estimated at approximately 76 years and for $R_{0}=0.9$ was estimated at 22 years. The time is greatly reduced to 4.5 years if $R_{0}=0.6$. Although our results show that eradication can be achieved by exclusively adding BCG badger vaccination, introducing added controls in cattle that can further reduce $R_{c c}$ and subsequently $R_{0}$, can also play a decisive role in the eradication programme as it will help to reduce the time to eradication; further, it could become a "deal breaker" for achieving it. To elucidate, as mentioned previously that badger culling is going to be reduced over time in Ireland, if badger culling is reduced, $R_{0}$ will increase over a number of years. Therefore, achieving a fast reduction of $R_{0}$ before 
seeing the effects of reduced/no culling in the NGM might be necessary to achieve eradication. Critical assessment of current controls as well as the introduction of new measures, such as risk based trading, a national policy on better management of infected herds and addressing residual infection, should be considered to assure the needed fast decline in $R_{0}$.

Finally, an important concept to communicate to policy makers is that $R_{0}$ is not a static parameter; as new control options are introduced, the NGM of the system will change, hence the need to re-assess this matrix over time. The relative simplicity in which the NGM was estimated in this thesis (not so much the way in which it was derived) will make this task easy and greatly recommended. For that, data on prevalence in both species as well as number of cattle herds and badgers should be collected on an ongoing basis during the eradication programme.

\section{Challenges ahead and suggestions for further work}

a) Defining the role of badger culling into the future

In Ireland, badger and cattle prevalence are not homogeneous throughout the country when measured in single cross sectional. Whether that implies that the NGMs described in this thesis for the country as a whole, will not apply depends on the long term average for these smaller areas. The conclusions from this thesis then might or might not be valid in these areas with larger/smaller badger and/or cattle prevalence. In order to decide the best eradication strategies in these areas, first it would be necessary to establish whether or not the system (in these areas) is at the endemic equilibrium and what the magnitude of this endemic equilibrium is. Defining the plausible NGMs for these areas will also provide information on the most adequate eradication strategies. Calculations based on the results from Chapter 6 show that $M$. bovis eradication would not be feasible with badger vaccination coverage equal to $40 \%$ if badger prevalence exceeds $23 \%$. A possible solution here would be to use focused/targeted badger culling (instead of vaccination), together with added cattle controls as an aid to bring badger density and prevalence down prior to the introduction of badger vaccination (assuming that the system here is at the endemic equilibrium and the conclusions from this thesis still apply).

In Ireland, some badger culling will remain in place once BCG badger vaccination is incorporated to the eradication programme (O'Keeffe, DAFM, personal communication). Also, it is important to clarify that the deployment of BCG vaccine in badgers in Ireland will be done intramuscularly as there is as yet no oral vaccine available (personal communication). If an oral vaccine was available, then deployment of the vaccine could be done in a timely manner assuring large vaccination coverage given adequate uptake levels. As the logistic efforts to deploy BCG vaccine intramuscularly will be lengthy and very similar to those required for badger culling, and as resources will be scarce, some policy will have to be written 
on when badger culling will occur instead of vaccination. Further work on population recovery and estimations of the effect of badger density on $R_{0}$ are recommended.

\section{b) Logistics of the transition from culling to vaccination}

Operational and policy changes will be needed to move to a new eradication programme that encompasses vaccination. Some of the logistical changes will include: the development of appropriate software to run the programme, staff training, etc. More importantly, if culling and vaccination are going to be used concurrently, specific criteria will have to be laid out as to when either of the two options are applied. Consistency in the criteria used will help in the assessment of programme progress.

\section{c) Maintaining a robust programme in the face of political pressure}

BCG vaccine efficacy in the field was estimated in Chapter 5. In this chapter, it was shown that during the four years the Kilkenny vaccine trial lasted, bTB prevalence in badgers did not decrease in any of the trial zones, including zones $\mathrm{B}$ and $\mathrm{C}$ where vaccination had been applied at 50 and $100 \%$ vaccination coverage. Although the real coverage achieved in these zones was lower than that quoted, and not sustained over time (see Figure 2 in Chapter 5), it is expected that a similar lack of decrease in prevalence will be observed in the years shortly after the introduction of a badger vaccination programme; this will result in disquiet among interested observers about the efficiency of the applied controls and will put considerable pressure on policy makers. Notwithstanding the lack of effect of vaccination on bTB prevalence in badgers in the short term, in Chapter 5 it was possible to demonstrate that during the study period, a reduction of badger to badger transmission occurred in the zones where vaccination had been applied (zones B and C), indicating that badger vaccination was working. Therefore, it is critical that adequate systems for measuring ongoing badger to badger transmission are designed, especially in the first few years after the introduction of badger vaccination. Also, in order to provide confidence that the added control options in cattle and badgers are working, data that allows estimation of changes in the cattle-badger system should be collected, and the NGM of the system assessed over time.

\section{Conclusion}

In Ireland, BCG badger vaccination has the potential to eradicate $M$. bovis infection not only from the badger population but also from the cattle-badger system. Although $\mathrm{BCG}$ vaccine efficacy in badgers is not high $\left(\mathrm{VE}_{\mathrm{S}}=59 \%\right.$ and $\left.\mathrm{VE}_{\mathrm{I}}=0 \%\right)$, we have demonstrated that the values the reproduction ratio for the system can take are not far from $1\left(1.07 \leq R_{0} \leq 1.16\right)$ and in most cases can be brought below 1 if BCG vaccination is added to the current controls in the country. Nonetheless, for the reasons explained in this chapter, we recommend that new, more stringent, and/or 
revised control options in cattle are adopted (along with badger vaccination) as a reassurance and as a way to speed up the eradication programme. Finally, it is greatly encouraged that policy makers understand the importance, and allow for adequate systems to measure progress to be put in place if/when additional control options are added. Also, it is important for all stakeholders to have realistic expectations from the badger vaccination programme, expectations which should always be based on science. The inability of achieving eradication of $M$. bovis in cattle in Ireland and other countries with a wildlife reservoir has generated large controversy. Now, and in view of the results in this thesis, it is possible to explain why, in countries without a wildlife reservoir, bTB has been eradicated quite efficiently using similar control strategies to the ongoing controls in cattle in Ireland. 


\section{References}

Aznar, I., McGrath, G., Murphy, D., Corner L.A.L., Gormley, E., Frankena, K., More, S.J., Martin, W., O’Keeffe, J., de Jong M.C.M., 2011. Trial design to estimate the effect of vaccination on tuberculosis incidence in badgers. Vet. Microbiol. 151, 104-111.

Byrne, A.W., O’Keeffe, J., Green, S., Sleeman, D..P, Corner L.A.L., Gormley, E., Murphy, D., Martin, S.W., Davenport, J., 2012. Population estimation and trappability of the European badger (Meles meles): Implications for tuberculosis management. PLoS ONE7(12), e50807. https://doi.org/10.1371/journal.pone.0050807

Byrne, A.W., Kenny, K., Fogarty, U., O'Keeffe, J.J., More, S.J., McGrath, G., Teeling, M., Martin, S.W., Dohoo, I.R., 2015. Spatial and temporal analyses of metrics of tuberculosis infection in badgers (Meles meles) from the Republic of Ireland: Trends in apparent prevalence. Prev. Vet. Med. 122, 345-354.

Costello, E., Flynn, O., Griffin, J., Quigley, F., O’Grady, D., McGrath, G., Hammond, R.F., 1999. Spatial distribution of RFLP types identified in Mycobacterium bovis isolates from badgers and cattle in a study area in the Republic of Ireland. In: Collins, J.D., Hammond, R.F. (Eds.), Selected Papers 1999. University College Dublin, pp. 26-30.

DAFM, Press/Information Office (2017). Creed comments on the results of badger vaccine trial [Press release]. Retrieved from:

https://www.agriculture.gov.ie/media/migration/press/pressreleases/2017/februa ry/PR172017020217.pdf. Accessed 24-10-2017.

EFSA AHAW Panel (EFSA Panel on Animal Health and Welfare), 2013. Scientific opinion on field trials for bovine tuberculosis vaccination. EFSA J. 11(12), 3475, $35 \mathrm{pp}$.

doi:10.2903/j.efsa.2013.3475.

Gormley, E., N1' Bhuachalla, D., O’Keeffe J., Murphy D., Aldwell F.E., Fitzsimons T., Stanley, P., Tratalos, J.A., McGrath, G., Fogarty, N., Kenny, K., More, S.J., Messam, L.L., Corner, L.A.L., 2017. Oral vaccination of free-living badgers (Meles meles) with Bacille Calmette Guérin (BCG) vaccine confers protection against tuberculosis. PLoS ONE 12, e0168851.

Griffin, J.M., Williams, D.H., Kelly, G.E., Clegg, T.A., O’Boyle, I., Collins, J.D., More, S.J., 2005. The impact of badger removal on the control of tuberculosis in cattle herds in Ireland. Prev. Vet. Med. 67, 237-266.

More, S.J., Good, M., 2006. The tuberculosis eradication programme in Ireland: a review of scientific and policy advances since 1988. Vet. Microbiol. 112, 239251.

O'Boyle, I., 2000. Review of badger (Meles meles) research licences in 2000 and 2001. University College Dublin. Centre for Veterinary Epidemiology and Risk Analysis, 2002-06. Available at: http://hdl.handle.net/10197/8865O' 

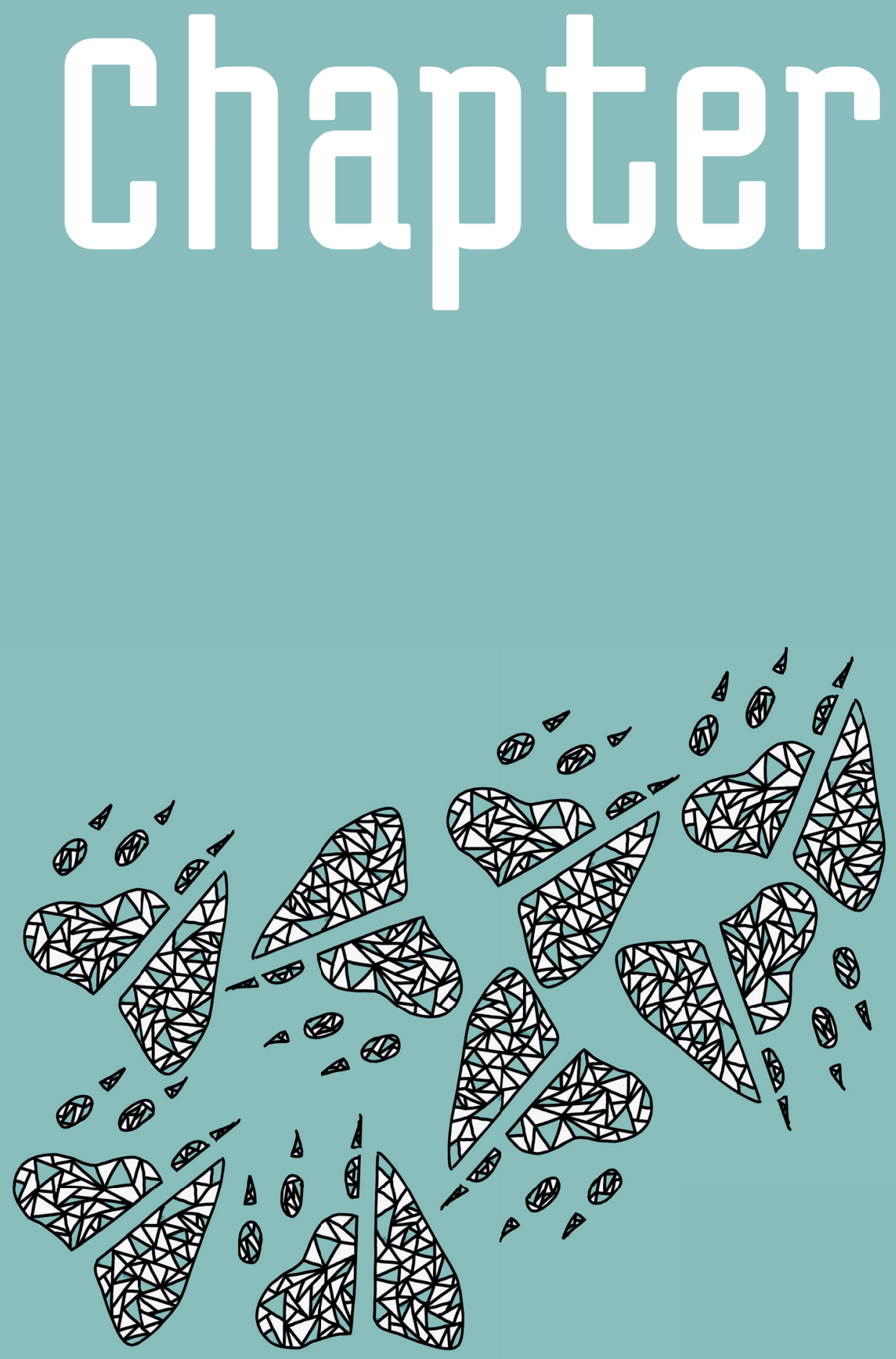


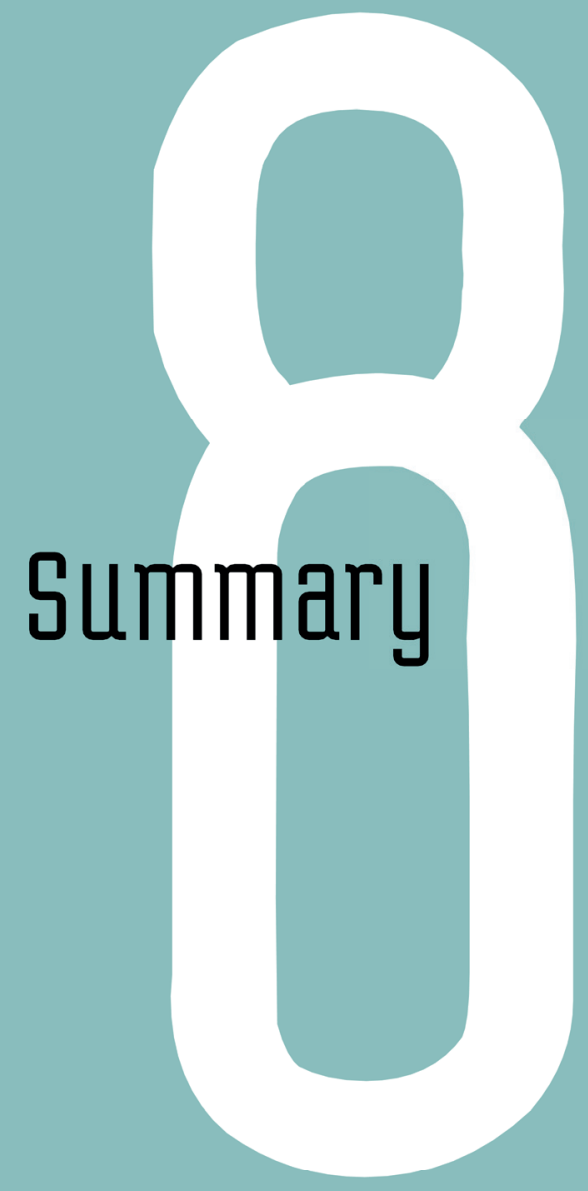


Bovine tuberculosis (bTB) is a chronic inflammatory disease of cattle caused by infection with Mycobacterium bovis. Apart from the respiratory and other general symptoms similar to those observed in human tuberculosis, cattle infected with $M$. bovis can show some specific symptoms such as mastitis. When tuberculous lesions develop in the udder, shedding of the mycobacteria via milk is possible. In the past, consumption of unpasteurized milk contaminated with this agent was a common cause of infection in humans. Although the number of human M. bovis infections has been greatly reduced in developed countries, contaminated milk and milk products still remain the main vehicle of transmission. The large decrease achieved in the number of human tuberculosis cases due to M. bovis, was mainly the result of control and eradication programmes in cattle, largely based (although not exclusively) on the testing and removal of infected animals. Some countries were successful in the eradication of the infection in cattle; however, Ireland and the UK are two countries where infection is still present despite ongoing control/eradication programmes over many years. Ireland commenced a control/eradication programme in 1954 , and by 1965 , cattle incidence had been reduced by more than $95 \%$ (from $17 \%$ to $0.5 \%$ ). Following scientific advice, the control programme was intensified over subsequent years yet cattle incidence remained relatively stable. The fact that some countries were able to eradicate the infection, while others struggled to maintain it at a low prevalence generated much controversy around the efficacy of the control strategies used in the latter group. In Ireland and the UK, further controversy was sparked by the discovery of a second host for M. bovis, the Eurasian badger (Meles meles). Based on subsequent research, badgers were identified as the main wildlife reservoir for infection in both countries. Although the exact role of badgers in transmission was (at the beginning of this thesis) still not known, several studies had confirmed a significant association between badger removal and a decrease in cattle bTB outbreaks. In Ireland, some targeted badger culling commenced in the early 1990s, and became part of the eradication programme some years later. Badger culling was considered a short-medium term strategy that would allow for research to be carried out on other plausible control strategies in the meantime. A number of laboratory studies where badgers were vaccinated with Bacillus Calmette-Guerin (BCG) vaccine, reported a reduction in disease progression in vaccinated compared to non-vaccinated badgers subsequent to being experimentally challenged with $M$. bovis. In Ireland, there were expectations that BCG vaccination of badgers in the field would help to reduce $M$. bovis transmission not only within the badger population but also between cattle and badgers. This $\mathrm{PhD}$ was designed to determine whether or not these expectations had a scientific base.

As a first step, assessing the impact of BCG vaccination in the field, rather than in an experimental context, was necessary. Although all badgers challenged with $M$. bovis in these trials became infected, some protection against infection could potentially be attained under natural conditions. The infective dose that badgers are 
exposed to in the field, the number of infection events to achieve this dose and the route of infection are all unknown. All these parameters, among others, could have an impact on the degree of BCG protection that is achieved in the field. In addition, the indirect effects of BCG vaccination in badgers had been never investigated; this indirect effect could potentially help in reducing transmission.

In the second chapter of this thesis, a badger BCG vaccine field trial was designed in a manner that allowed for the effects of BCG vaccination on badger to badger transmission to be assessed. For that, two types of vaccine efficacy had to be estimated, vaccine efficacy for susceptibility $\left(V_{S}\right)$ and vaccine efficacy for infectiousness $\left(\mathrm{VE}_{\mathrm{I}}\right)$. $\mathrm{VE}_{\mathrm{S}}$ measures the direct effect of vaccination achieved as a reduction in the susceptibility of badgers to $M$. bovis infection. On the contrary, $\mathrm{VE}_{\mathrm{I}}$ is a measure of a type of indirect effect, resulting from a reduction in the infectiousness of vaccinated and subsequently infected badgers. A double blind $(50 / 50)$ vaccine/placebo randomized control trial is regularly used to assess the direct effects of vaccination; however, this type of design does not allow for estimations of $\mathrm{VE}_{\mathrm{I}}$. The design of choice was therefore a compromise whereby both effects could be obtained. More importantly, by estimating the effects of vaccination on both susceptibility and infectivity, it was possible to assess the effects of the vaccine on badger to badger transmission. The study area (located in County Kilkenny in Ireland) of approximately 750 square $\mathrm{km}$ was divided from North to South into three zones (A, B and C) with similar characteristics in terms of size, number of main badger setts, cattle herds, cattle and land classification type. Vaccination was applied at 0,50 and $100 \%$ coverage with badgers in the middle zone being randomly allocated to the vaccine/placebo, and all badgers in zone A receiving the placebo. In this chapter the methodology for analysing the trial data collected was also explained.

The third chapter of this thesis was dedicated to assess the power of the study design. Here, the effects on study power of sample size, prevalence in badgers at the beginning of the trial, sensitivity and specificity of the diagnostic test, transmission rate between unvaccinated badgers, $\mathrm{VE}_{\mathrm{S}}$ and $\mathrm{VE}_{\mathrm{I}}$ were determined. The main finding from this study was that the specificity of the diagnostic test used in the trial had a very large effect on the study power, while test sensitivity did not play a large role. Specificity values below $99.4 \%$ resulted in study power below $50 \%$ as even a small proportion of false positives, randomly occurring in the many negative samples from both vaccinated and unvaccinated animals masked the effects of vaccination. It was concluded then that the diagnostic test to be used in the badger vaccine trial had to be optimized to maximize test specificity.

In the fourth chapter the characteristics of a multiplex chemiluminescent assay (Enfer test) for detection of M. bovis infection in badgers were optimized in order to maximise specificity, and therefore statistical power. The Relative Light Units 
(RLU) obtained from testing blood samples from badgers captured by the national Department of Agriculture, Food and the Marine (DAFM) were analysed. A stepwise logistic regression with analytical weights was used for the analysis. Optimisation resulted in a test with $30.77 \%$ sensitivity and $99.99 \%$ specificity. In addition, and significant for the analysis, the characteristics of this test did not vary greatly between vaccinated and non-vaccinated badgers.

The results from the BCG badger vaccine trial were presented in the fifth chapter. Badgers in the three zones of the vaccine trial area were tattooed and microchipped at the first trapping, with trappings occurring over four years. Blood samples were collected at first capture and every subsequent recapture. Data obtained from the three zones of the vaccine trial, as well as blood test results, were analysed with the aim to compare new infections occurring in non-infected non-vaccinated badgers to those in non-infected vaccinated ones. A Generalized Linear Model (GLM) with a cloglog link function was used for the analysis. Vaccine efficacy for susceptibility was estimated as $\mathrm{VE}_{\mathrm{S}}=59 \%(95 \% \mathrm{CI}=6.5 \%-82 \%)$ whereas vaccine efficacy for infectivity $\left(\mathrm{VE}_{\mathrm{I}}\right)$ was $0 \%$, implying that there was a complete lack of effect from BCG vaccination on the infectivity of vaccinated and subsequently infected badgers. In this chapter, the impact of badger vaccination on badger transmission was also assessed for the first time. For that, the reproduction ratio for badger to badger transmission $\left(R_{0}\right)$ under current controls was estimated using a simple formula as 1.22 assuming a prevalence in badgers of $18 \%$. Therefore, and as BCG vaccination was shown to be able to reduce transmission by $59 \%$, the reproduction ratio for a badger population vaccinated with $100 \%$ vaccine coverage (or $R_{1}$ ) was estimated to be 0.5. These results suggested that eradication in badgers by adding BCG vaccination to the current controls in cattle and badgers would be feasible. The formula used for estimating $R_{0}$ assumes that there is no transmission between cattle and badgers. Based on available research, this was unlikely to be the case which suggests that these last results could only be taken as an approximation. Consequently, the transmission between the two species was explored in the final chapter.

In Chapter 6, and as the culmination of the previous chapters, the quantitative role of each of the two host species, cattle and badgers, in the transmission of M. bovis in Ireland was assessed. Algorithms to estimate the Next Generation Matrix (NGM) and the basic reproduction ratio for the cattle-badger system were developed and all plausible NGMs describing the transmission between the two species were calculated from prevalence data and the number of cattle herds and badgers, collected at the endemic steady state. By exploring the system at the endemic equilibrium, it was possible to demonstrate that for almost all these plausible transmission scenarios, BCG vaccination of badgers in addition to current controls could successfully achieve eradication of $M$. bovis infection in the system. All 
transmission scenarios were represented within a NGM triangle, with the vertices of this triangle representing extreme transmission possibilities. The small number of transmission scenarios where eradication of $M$. bovis from the system by adding BCG badger vaccination to the current controls was not feasible were shown as an area within the triangle. These scenarios were characterized by small/non-existent transmission from badgers to cattle, with all or almost all of the infections in cattle and badgers being caused by cattle. These scenarios would be highly unlikely to represent transmission in the field, and only represent a small proportion of the total. Similar results were obtained when the effects of a smaller badger vaccination coverage $(40 \%)$ was investigated. It was concluded therefore, that BCG vaccination of badgers has the potential, if added to current controls, to achieve eradication of $M$. bovis infection in the cattle-badger system in Ireland. Nonetheless, for reasons explained in the general discussion (Chapter 7) of this thesis, it is highly recommended that additional/more stringent controls are applied in cattle along with BCG badger vaccination in order to ensure and/or accelerate the eradication process. In this final chapter, some answers to potential queries in relation to adding BCG vaccination to the current eradication programme, and some further recommendations for policy makers and other stakeholders are presented. As stated in the final paragraph in Chapter 7: "Now, and in view of the results in this thesis, it is possible to explain why, in countries without a wildlife reservoir, bTB has been eradicated quite efficiently using similar control strategies to the ongoing controls in cattle in Ireland.". 


\section{Training \& Supervision Plan (TSP)}

The Basic Package (3.0 ECTS)

Year

WIAS Introduction course

2008

Ethics course

2010

\section{Scientific Exposure (15.5 ECTS)}

International conferences

Society for Veterinary Epidemiology and Preventive 2008

Medicine, Liverpool (UK)

M. bovis V Conference, Wellington, (NZ) 2009

Society for Veterinary Epidemiology and Preventive 2009

Medicine, London (UK)

Society for Veterinary Epidemiology and Preventive

2010

Medicine, Nantes (FR)

Society for Veterinary Epidemiology and Preventive

2011

Medicine, Leipzig (DE)

Society for Veterinary Epidemiology and Preventive

Medicine, Dublin (ROI)

M. bovis VI Conference, Cardiff, Wales (GB)

Society for Veterinary Epidemiology and Preventive

Medicine, Ghent (BE)

Society for Veterinary Epidemiology and Preventive

Medicine, Aberdeen (GB)

Presentations

$M$. bovis $V$ Conference, Wellington (NZ), oral

2009

WIAS Science Day, Wageningen (NL), oral

2010

Society for Veterinary Epidemiology and Preventive 2011

Medicine, Leipzig (DE), oral

M. bovis VI Conference, Cardiff, Wales (GB), oral 2014

Seminar and workshops

WIAS Science Day, Wageningen (NL)

"Epi-mentoring group" workshop, organized on a

yearly basis (ROI)

TB training for Veterinary Inspectors (ROI)

2017

\section{In-Depth Studies (6.0 ECTS)}

Disciplinary and interdisciplinary courses

Mathematical modelling in biology, Wageningen (NL) 2008

Mathematica (self-taught) 2011

Advanced statistics courses 
Professional Skills Support Courses (3.7 ECTS)

Writing scientific proposals

Presentation skills enhancement

Senior management

\section{Research Skills Training (6.0 ECTS)}

Preparing own $\mathrm{PhD}$ research proposal

* one credit equals a study load of 28 hours 


\section{Thesis Contributions}

- This research was carried out under direct supervision from Mart C.M de Jong and Klaas Frankena (Quantitative Veterinary Epidemiology unit, Wageningen University and Research). They both contributed to shaping the main research questions and designing a pathway for reaching answers in a scientifically proofed manner. Within each chapter, they provided support in terms of study design, statistical and mathematical modelling as well as advice in the presentation of results and conclusions. They also provided guidance in terms of complying with all regulations related to the thesis, including the training and supervision plan.

- This research project was requested by the Department of Agriculture, Food and the Marine (DAFM) after consultation with experts in the field, including: Simon More (Professor of Epidemiology, School of Veterinary Medicine, UCD), Eamonn Gormley (Associate Professor, School of Veterinary Medicine, UCD), Leigh Corner (Researcher associated with the School of Veterinary Medicine, UCD), Wayne Martin (Professor Emeritus of Epidemiology, Department of Population Medicine, University of Guelph), and Professor Mart de Jong (Promotor).

- An initial design of the badger vaccine trial described in this thesis was outlined by Eamonn Gormley and Leigh Corner. This design was subsequently modified by the author in collaboration with all those mentioned above.

- The field work, both in preparation and during the badger vaccine trial, was organized and led by Eamonn Gormley and Leigh Corner (UCD), and James O'Keeffe (DAFM). Eamonn Gormley, Denise Murphy (Research Officer, DAFM) and Leigh Corner were also responsible for post-mortem, histopathology and culture of badger tissue samples.

- Enfer Scientific carried out the diagnostic assays (Enferplex) used for determining the sero-status of badgers with regard to Mycobacterium bovis infection.

- Professor Simon More and Professor Wayne Martin provided epidemiological support throughout the research. Professor Simon More provided advice in the structure and content of the discussion. CVERA (Centre for Veterinary Epidemiology and Risk Analysis) staff provided support in terms of data analysis, GIS and art work.

- The project was funded by DAFM, with Michael Sheridan (Deputy Chief Veterinary Officer) and James O'Keeffe (Superintendent Veterinary Inspector) overseeing the research project.

- The front cover was designed by Maria Cos and J. Luz Aznar 


\section{Colophon}

-Funding: This project was funded by DAFM (Department of Agriculture, Food and the Marine), Ireland.

-Cover design and lay-out: Maria Cos and J. Luz Aznar. 


\section{Propositions}

1. Despite BCG vaccination being classified as a having a medium/low vaccine efficacy, a relatively low coverage vaccination programme can achieve $M$. bovis eradication in the cattle-badger system in Ireland.

(this thesis)

2. The days of the 50:50 vaccine/placebo randomized trials are over. (this thesis)

3. The average person is not as afraid of antimicrobial resistance as he should be.

4. Europe is at risk of increased transmission of vaccine-preventable diseases due to the current migration crisis.

5. Social media are key in the failure or success of vaccination campaigns.

6. The increase in life expectancy is being used as an excuse for increasing retirement age.

Propositions belonging to the thesis, entitled:

Infection dynamics and effective control strategies of tuberculosis in badgers and cattle in Ireland.

J Inmaculada Aznar Asensio

Wageningen, 9 February 2018 